This item was submitted to Loughborough's Research Repository by the author.

Items in Figshare are protected by copyright, with all rights reserved, unless otherwise indicated.

\title{
Drop mixing in suspension polymerisation
}

PLEASE CITE THE PUBLISHED VERSION

\section{PUBLISHER}

Shahrir Hashim

\section{PUBLISHER STATEMENT}

This work is made available according to the conditions of the Creative Commons Attribution-NonCommercialNoDerivatives 4.0 International (CC BY-NC-ND 4.0) licence. Full details of this licence are available at: https://creativecommons.org/licenses/by-nc-nd/4.0/

\section{LICENCE}

CC BY-NC-ND 4.0

\section{REPOSITORY RECORD}

Hashim, Shahrir. 2018. "Drop Mixing in Suspension Polymerisation”. figshare.

https://hdl.handle.net/2134/34911. 
Loughborough University

\section{Pilkington Library}

Author/Filing Title ......................................

Vol. No. ........... Class Mark .......................

Please note that fines are charged on ALL overdue items.

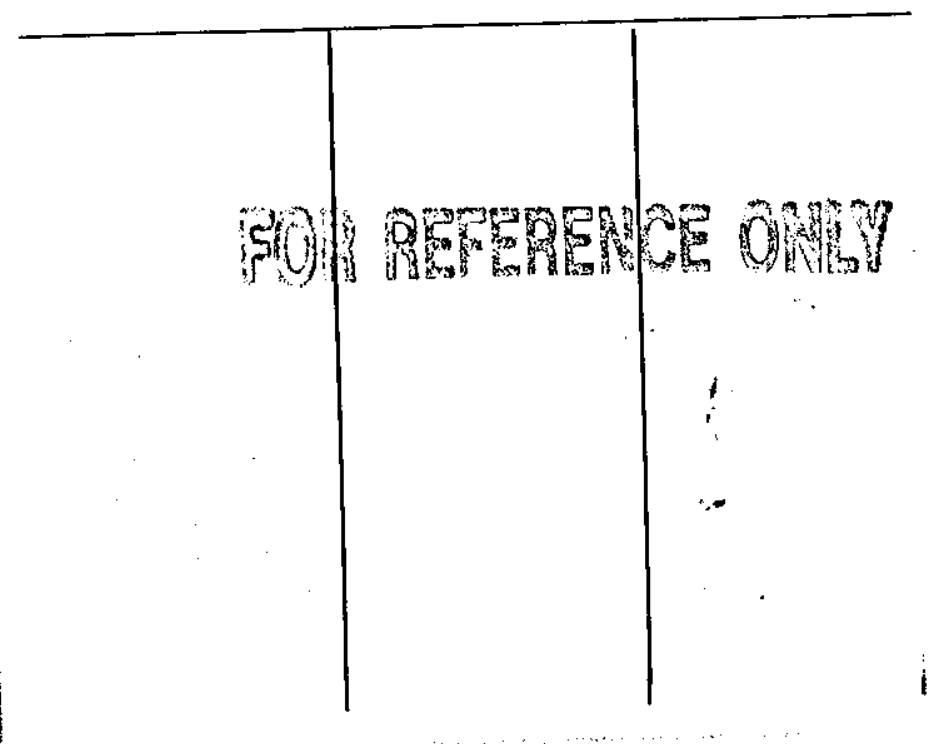





\title{
DROP MIXING IN SUSPENSION POLYMERISATION
}

\author{
BY \\ SHAHRIR HASHIM
}

A Doctoral Thesis

Submitted in partial fullfillment of the requirements for the award of the Doctor of Philosophy of the Loughborough University

February 2001 


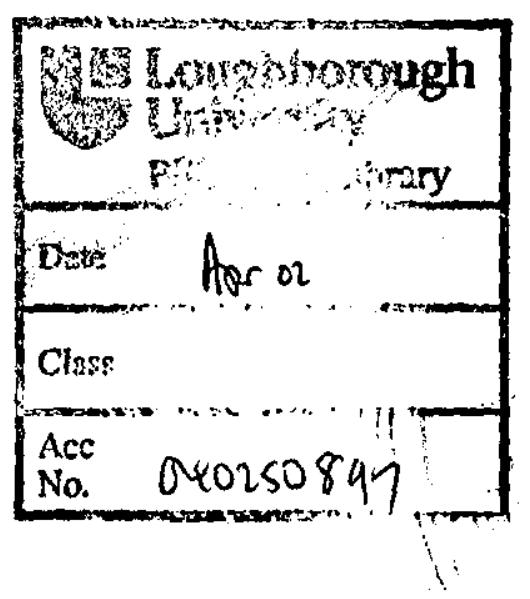




\section{ACKNOWLEDGEMENT}

I wish to express my sincere gratitude and appreciation to my supervisor, Professor B.W. Brooks for all his guidance, ideas, encouragement and patient he gave during the completion of this work. Grateful appreciation is also extended to my director of research, Professor B.A. Buffham.

Sincere thanks to Andy Milne for his help with the practical aspects of this work. I would also like to acknowledge the assistance of Steve Graver and Martin Kerry.

My appreciation also to the Malaysian Government and Univerisiti Teknologi Malaysia for granting me the scholarship.

No list of acknowledgement would be completed without mentioning my beloved parents, Hashim Abdullah and Che Su Ibrahim, who have been very concerned for this task to be completed.

To my beloved wife, Norhayati, thanks for loving moral support and encouragement throughout the completion of this work. To my four children, Fathi, Norafifah, Norsyahirah and Tasnim, thanks for your patience and understanding. 


\section{Summary}

Suspension polymerisation is one of the major processes used to produce polymers. In this process, monomer is suspended as liquid droplets in a continuous water phase by mean of strong agitation and the presence of a suspending agent. As the suspension polymerisation proceeds, the viscosity of a monomer-polymer droplet increases with conversion. Hence, the physical behaviour of the droplet changes during the process. When new dispersible material is added to the existing suspension drops, the new material and existing drops can remain segregated for significant amounts of time. This will affect the properties of polymer products.

The aim of this project was to study the behaviour of drop mixing when new material is added to the existing suspension polymerisation. This study concentrated on the effect of the dispersed phase viscosity on the drop mixing, but agitation intensity and surface stabilities were also important.

Experiments were carried out using a simulated dispersed-phase material. Polystyrene-styrene solutions with different viscosities were used as dispersed phase for this purpose. The technique used to determine the extent of mixing between the drops was the tracer dye technique. The apparatus used was a glass reaction vessel with a curved bottom. The drops were analysed by using a microscope with an attached video system together with a monitor. Two types of mixing were done. In the first type, an unstabilised batch of monomer was added to the existing dispersion, in the second type, two stabilised dispersions were mixed. Different surfactants were also used in order to study the effect of the type of surfactant on the drop mixing.

The results show that viscosity affects the rate of coalescence between drops. As the viscosity increased, the rate of coalescence decreased. Meanwhile, the viscosity also increases the drop size, which also affects the coalescence rate. It was found that at low drop size, as the viscosity increased, the rate of coalescence increased. However at high drop size, drop size does not influence the coalescence rate. For the mixing of two dispersions with the same viscosity of dispersed phase, it was found that the drop size distribution remains the same as that before the mixing. Attempts were also made to compare the behaviour of the drops with prediction of mixing models. 


\section{TABLE OF CONTENTS}

LIST OF TABLES

LIST OF FIGURES vi vi

LIST OF SYMBOLS ix

CHAPTER 1 - INTRODUCTION AND OBJECTIVES 1

1.1 General Introduction 1

1.2 Objectives 2

1.3 Thesis Organisation 4

CHAPTER 2 - LITERATURE REVIEW

2.1 Liquid-liquid dispersion 5

2.1.1 Effect of dispersed phase viscosity 6

$\begin{array}{ll}2.1 .2 \text { Effects of surfactant } & 8\end{array}$

2.2 Turbulent mixing theory 9

2.3 Physical properties of liquid-liquid dispersion 11

2.4 Drop Size Correlation 12

$\begin{array}{lll}2.4 .1 & \text { Maximum Stable Drop Size } & 13\end{array}$

$\begin{array}{ll}\text { 2.4.2 Minimum Stable Drop Size } & 17\end{array}$

$\begin{array}{lll}\text { 2.4.3 Simultaneous Break-up and Coalescence } & 18\end{array}$

$\begin{array}{lll}2.4 .4 & \text { Drop Size Distribution } & 19\end{array}$

2.5 Breakage and Coalescence Rate 21

$\begin{array}{lll}2.5 .1 & \text { Breakage rate } & 22\end{array}$

$\begin{array}{ll}2.5 .2 \text { Coalescence Rate } 25 & 25\end{array}$

2.6 Previous Experimental Approach 31

2.7 Interfacial Phenomena $\quad 32$

$\begin{array}{lll}2.7 .1 & \text { Surfactant System } & 33\end{array}$

2.8 Polymerisation Processes 36

2.8.1 Bulk (mass) Polymerisation $\quad 37$

$\begin{array}{ll}\text { 2.8.2 Solution Polymerisation } & 37\end{array}$

2.8.3 Emulsion Polymerisation $\quad 38$

$\begin{array}{lll}2.8 .4 & \text { Suspension Polymerisation } & 39\end{array}$ 
2.8.4.1 Types of Suspension Polymerisation 40

2.8.4.2 The Process of Suspension Polymerisation 41

2.8.4.3 Advantages and disadvantages of the suspension $\quad 42$ polymerisation

2.8.4.4 Suspending agents $\quad 42$

2.9 Free Radical Chain Polymerization 43

2.9.1 Mechanism 43

2.9.2 Overall Polymerisation Rate 46

2.9.3 Degree of Polymerisation $\quad 47$

$\begin{array}{lll}2.9 .4 & \text { The gel effects } & 47\end{array}$

2.9.5 Copolymerisation 48

CHAPTER 3 - EXPERIMENTAL WORK 50

$\begin{array}{lll}3.1 & \text { Apparatus } & 50\end{array}$

3.1.1 Liquid-liquid dispersion apparatus $\quad 50$

3.1.2 Apparatus for analysis of drops $\quad 59$

3.1.3 Characterisation apparatus $\quad 61$

3.2 Materials 61

3.2.1 Dispersed Phase $\quad 61$

3.2.2 Continuous Phase 62

3.3 Experimental Methods 63

3.3.1 Preparation of dispersed phase 63

3.3.2 Preparation of surfactant solution $\quad 63$

3.3.3 Preparation of dyed solution $\quad 63$

3.3.4 Drop size measurement 64

3.4 Specific Experimental Methods 65

3.4.1 Preliminary study on drop mixing 65

3.4.1.1 Study of batch mixing of toluene with stabilised 66 dispersion of polystyrene solution

3.4.1.2 Study of extent of mixing between two dispersions 67

3.4.2 Colour contrast observation on the optical microscope 68

3.4.3 Study of batch mixing of styrene with stabilised 69 dispersion of polystyrene solution 
3.4.3.1 Premixing behaviour of polystyrene solution dispersion $\quad 70$

3.4.3.2 Batch mixing of styrene with polystyrene solution 70

3.4.4 Study of mixing of two stabilised dispersions 71

3.4.4.1 Study of the mixing of same viscosities of dispersed phase $\quad 72$

3.4.4.2 Study of the mixing of different viscosities of dispersed phase 73

3.4.3.3 Study of comparison between batch mixing and mixing $\quad 74$ of two dispersions

CHAPTER 4 - RESULTS AND DISCUSSION 75

4.1 Preliminary study on drop mixing 75

4.1.1 Drop mixing for added batches of toluene to a stabilised 76 dispersion with a different dispersed phase viscosity

4.1.2 Study of the extent of mixing between two dispersions $\quad 78$

4.1.2.1 Extent of mixing for two different surfactants 78

4.1.2.2 Extent of mixing for two different conditions using 81 0.02 wt \% Tween 20

4.2 Batch mixing of styrene with stabilised dispersion of polystyrene $\quad 83$ solution

4.2.1 Dispersion behaviour of the dispersed phase before mixing of new batch

4.2.2 Drop mixing between a new batch of styrene and the existing stabilised dispersion using $0.02 \%$ PVA

4.2.2.1 Drop mixing at $500 \mathrm{rpm}$

4.2.2.2 Drop mixing at $650 \mathrm{rpm} \quad 95$

4.2.2.3 Drop mixing at $350 \mathrm{rpm} \quad 98$

$\begin{array}{ll}\text { 4.2.3 Effect of stirring speed on batch mixing } & 102\end{array}$

4.2.4 Effect of concentration of PVA on batch mixing 107

4.2.5 Effect of type of suspending agent on batch drop mixing $\quad 110$

$\begin{array}{lll}4.3 & \text { Mixing of two stabilised dispersion } & 117\end{array}$

$\begin{array}{ll}\text { 4.3.1 Mixing of same viscosities of dispersed phase } & 117\end{array}$

4.3.1.1 Comparisons between dispersed mixing and batch mixing 133

4.3.2 Mixing of different viscosities of dispersed phases 141 


\section{WITH LITERATURE MODEL}

5.1 Introduction

5.2 Experimental results

5.3 Coalescence rate model 153

$\begin{array}{lll}\text { 5.3.1 Coalescence rate } & 154\end{array}$

$\begin{array}{lll}\text { 5.3.2 Coalescence efficiency } & 155\end{array}$

CHAPTER 6-CONCLUSION AND RECOMMENDATION 162

6.1 Conclusion 162

$\begin{array}{lll}6.2 & \text { Recommendation } & 166\end{array}$

CHAPTER 7- BIBLIOGRPHY AND REFERENCES 168

$\begin{array}{lll}6.1 & \text { Bibliography } & 168\end{array}$

$\begin{array}{lll}6.2 & \text { References } & 169\end{array}$

$\begin{array}{ll}\text { APPENDIX } & 178\end{array}$

$\begin{array}{ll}\text { Appendix A } & 178\end{array}$

$\begin{array}{ll}\text { Appendix B } & 180\end{array}$

$\begin{array}{ll}\text { Appendix C } & 182\end{array}$

$\begin{array}{ll}\text { Appendix D } & 210\end{array}$ 


\section{LIST OF TABLES}

Table 3.1 Some physical properties of toluene

Table 3.2 Some physical properties of toluene

Table $3.3 \quad$ Viscosity of styrene/toluene solution

Table 3.4 Experimental condition for colour contrast experiments

Table $3.5 \quad$ Viscosity of styrene/polystyrene solution

Table 4.1 Reynold number for different viscosities of dispersed phase

Table 5.1 Experimental data for overall average drop diameter, extent of mixing and initial coalescence frequency

Table 5.2 Collision rate of different dispersed phase of mixing of two dispersions

Table 5.3 Kinematic viscosity of suspension for different viscosity of dispersed phase

Table 5.4 Interfacial tension of the drops in the different viscosity of dispersed phase

Table 5.5 Drop Reynold number and drop Weber number for different viscosity of dispersed phase

Table 5.6 Values of $Y_{o}$ for different concentration of dispersed phase

Table 5.7 The film drainage Weber number for different viscosity of dispersed phase

Table 5.8 Comparison between the result of the model and experiment for $10 \mathrm{wt}$ $\%$ polystyrene 


\section{LIST OF FIGURES}

Figure 2.1 Schematic diagram of the process of generation of turbulence and coalescence of drops

Figure 2.2 Orientation of surfactant molecules at the interface of droplet and continuous phase

Figure 3.1 Schematic diagram of reaction vessel

Figure 3.2a Schematic diagram of baffles

Figure $3.2 \mathrm{~b}$ Schematic diagram of stirrer

Figure 3.3 Schematic set-up diagram of batch mixings

Figure 3.4 Schematic diagram for fittings through the five-neck glass vessel lid (Batch mixing experiments)

Figure 3.5 Schematic set-up diagram of mixing of two dispersions experiments

Figure 3.6 Schematic diagram for fittings through glass lids in mixing of two dispersion experiments

Figure 3.7 Schematic diagram for the set-up of the microscope with the video system

Figure 3.8 Equipments for analysis of drops

Figure 4.1 Batch mixing of new toluene with the existing dispersed phase using 0.02 wt \% PVA

Figure 4.2 Extent of mixing between two dispersion of different surfactant

Figure 4.3a Drop size distribution for mixing of dispersion without stabiliser and dispersion using $0.02 \mathrm{wt} \%$ at 120 minutes

Figure 4.3b Drop size distribution for mixing of dispersion without stabiliser and dispersion using $0.02 \mathrm{wt} \%$ Tween 20 at 120 minutes

Figure 4.4 Extent of mixing for two different condition of mixing

Figure 4.5 Mean drop size as function of stirring time for different polystyrene solution

Figure 4.6 Drop size distribution of different polystyrene solution at 120 minutes

Figure 4.7 Drop mixing between coloured and uncoloured drops for $15 \mathrm{wt} \%$ polystyrene at 3 different stirring time

Figure 4.8 Extent of mixing for batch mixing of new styrene with different viscosities of dispersed phase

Figure 4.9 Drop size distribution for batch mixing at 180 minutes 
Figure 4.10 Drop size distributio for batch mixing of styrene with $20 \mathrm{wt} \%$ PS dispersed phase

Figure 4.11 Mean drop size for coloured and uncoloured drops at 15 wt \% PS and $20 \mathrm{wt} \%$ dispersed phase at $500 \mathrm{rpm}$

Figure 4.12 Extent of mixing for batch mixing of new styrene with different viscosities of dispersed phase at $650 \mathrm{rpm}$

Figure 4.13 Drop size distribution for batch mixing of styrene with $15 \mathrm{wt} \%$ PS dispersed phase at $650 \mathrm{rpm}$

Figure 4.14 Extent of mixing for batch mixing of new styrene with different viscosities of dispersed phase at $350 \mathrm{rpm}$

Figure 4.16 Extent of mixing for 15 wt \% PS dispersed phase running at different stirrer speeds

Figure 4.17 Drop size distribution at 5 minutes for $15 \mathrm{wt} \%$ polystyrene solution dispersed phase at different stirring speeds

Figure 4.18 Drop size distribution at 180 minutes for $15 \mathrm{wt} \%$ polystyrene solution dispersed phase at different stirring speed

Figure 4.19 Extent of mixing for batch mixing using $0.05 \mathrm{wt} \%$ PVA at $500 \mathrm{rpm}$

Figure 4.20 Extent of mixing for batch mixing using two different concentration of PVA

Figure 4.21 Drop size distribution at 180 minutes of batch mixing for two different of PVA

Figure 4.22 Extent of mixing for batch mixing using 0.02 wt $\%$ Tween 20 for different viscosity of dispersed phase

Figure 4.23 Extent of mixing for batch mixing at $15 \mathrm{wt} \%$ PS dispersed phase using different type of surfactant

Figure 4.24 Drop size distribution for batch mixing at 180 minutes using different surfactant

Figure 4.25a Drop size distribution for premixing after 120 minutes for 0 wt $\%$ polystyrene solution (styrene) dispersed phase

Figure 4.25b Drop size distribution for premixing after 120 minutes for $10 \mathrm{wt} \%$ polystyrene solution (styrene) dispersed phase.

Figure 4.26 Extent of mixing for mixing of two stabilised dispersion with different viscosities of dispersed phase 
Figure 4.27 Drop size distribution at 5 minutes at different viscosity of dispersed phase for mixing of two dispersion

Figure 4.28 Drop size distribution at 180 minutes at different viscosity of dispersed phase for mixing of two dispersion

Figure 4.29 Drop size distribution for mixing of two stabilised dispersion of styrene (0 wt \% PS) dispersed phase

Figure 4.30 Drop size distribution for mixing of two stabilised dispersion of $10 \mathrm{wt}$ $\%$ Polystyrene solution dispersed phase

Figure 4.31 Extent of mixing for dispersed mixing and batch mixing (0 wt \% PS + 0 wt \% PS)

Figure 4.32 Drop size distribution for batch mixing of $0 \mathrm{wt} \%+0 \mathrm{wt} \%$

Figure 4.33 Extent of mixing for dispersed phase mixing and batch mixing (10 wt $\%+10 \mathrm{wt} \% \mathrm{PS})$

Figure 4.34 Drop size distribution for batch mixing of $10 \mathrm{wt} \%+10 \mathrm{wt} \%$

Figure 4.35 Extent of mixing for two dispersion of different viscosity of dispersed phase

Figure 4.36 Extent of mixing for two dispersion of different viscosity of dispersed phase

Figure 4.37: Extent of mixing for two dispersion of different viscosity of dispersed phase

Figure 4.38 Drop size distribution for mixing of two stabilised dispersion (0 wt \% PS+ 0 wt \% PS)

Figure 4.39 Drop size distribution for mixing of two stabilised dispersion ( 0 wt \% + 0 wt \%) 


\section{LIST OF SYMBOLS}

$A=$ Interfacial area per unit volume $\mathrm{A}$

$A\left(h_{o}\right)=$ constant

$A(v) d v=\quad$ volume of the drops of size $v$.

$C=$ Concentration of surface active agent in the solution

$C_{i}=$ proportionality constant

$d \quad=\quad$ Drop diameter

$D=$ Impeller diameter

$d_{32}=$ Sauter mean diameter

$d_{e}=$ Eddy diameter

$D F(\phi)=\quad$ Turbulent damping factor

$d_{m}=$ Mean drop diameter

$d_{\max }=$ Maximum drop diameter

$d_{\min }=$ Minimum drop diameter

$d_{o}=$ Sauter mean diameter for non-viscous

$D P_{n}=$ Degree of polymerisation

$E_{a}=$ Adhesion energy

$E_{c}=$ Energy required for breakage

$E_{d}=$ Dispersed phase elasticity modulus

$E_{m} \quad=\quad$ Mixture of dispersed and continuous phase elasticity modulus

$E_{p}=$ Dispersed phase elasticity modulus

$E_{s}=$ Energy due to the interfacial tension

$E_{v}=$ Turbulent fluctuation energy

$f \quad=\quad$ Initiator efficiency factor

$f(\phi)=$ Correlation factor

$F \quad=\quad$ Volume of coloured drops coalescing with uncoloured drops per unit time

$F_{o}=$ Initial coalescence rate

$g(v)=$ number of drops of size $v$ breaking per unit volume of dispersion per unit time

$\Delta G_{\text {electrosstatic }}=$ Energy due to electrostatic forces of repulsion

$\Delta G_{\text {steric }} \quad=\quad$ Energy due to the steric effects (is a repulsion force) 


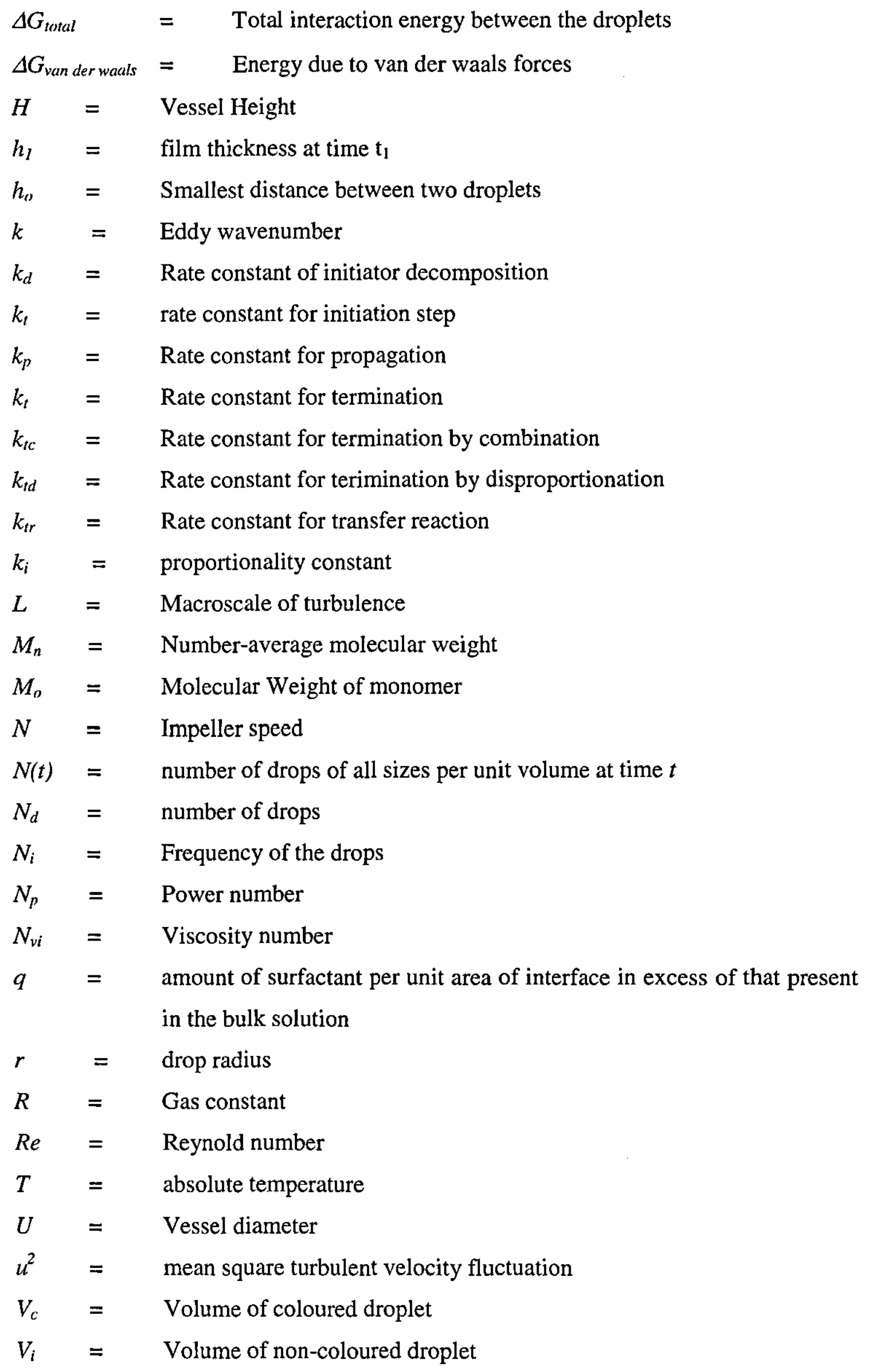




$$
\begin{array}{lll}
V & = & \text { Reactor volume } \\
v_{\max } & = & \text { maximum drop size present in the dispersion } \\
w_{*} & = & \text { critical approach velocity } \\
W e & = & \text { Weber number } \\
(W e)_{\text {crit }} & = & \text { Critical weber number } \\
X & = & \text { Extent of mixing }
\end{array}
$$

\section{Greek}

$\kappa\left(v, v^{\prime}\right) d v^{\prime}=$ the number of pairs of drops of size $v$ and $v^{\prime}$ coalescing per unit volume of dispersion per unit time.

$\omega_{b}(x)=\quad$ Eddy-drop collision frequency of a drop having volume $\mathrm{x}$

$\theta=\quad$ Volume of drops colliding per unit time

$\omega_{c}(x, y)=\quad$ Collision frequency of two drops having volumes $\mathrm{x}$ and $\mathrm{y}$

$\beta\left(v^{\prime}, v\right) d v=$ the fraction of droplets with volume $v$ formed by the breakage of a drop of volume $v^{\prime}$.

$\varepsilon=$ Energy dissipation rate per unit mass of fluid

$\phi \quad=\quad$ Dispersed phase ratio

$\gamma=$ Velocity scales

$\eta \quad=\quad$ Kolmogoroff length

$\lambda_{b}=$ Breakage efficiency function

$\lambda_{c}=$ Coalescence efficiency function

$\mu_{c}=$ Viscosity of continuous phase

$\mu_{d} \quad=\quad$ Viscosity of dispersed phase

$\mu_{m} \quad=\quad$ Viscosity of mixture of dispersed and continuous phase

$v \quad=\quad$ Kinematic viscosity of dispersion

$\rho_{c} \quad=\quad$ Density of continuous phase

$\rho_{d}=$ Density of dispersed phase

$\rho_{m} \quad=\quad$ Density of the mixture of continuous and dispersed phase

$\sigma=$ Interfacial tension between two immiscible liquid

$v\left(v^{\prime}\right)=$ the number of daughter particles resulting from a breakage of a drop of volume $v^{\prime}$. 


\section{CHAPTER 1}

\section{INTRODUCTION AND OBJECTIVES}

\subsection{General Introduction}

Liquid-liquid dispersions are very common operations in chemical industrial processes. Generally, multiphase processes are used in order to increase the interfacial area between the different phases to improve the interphase transport rates. Examples of liquid-liquid dispersion operations are liquid-liquid extraction, emulsification, suspension polymerisation and multiphase reaction. Usually, one liquid is dispersed as drops in a second liquid. Depending on the nature of the process, chemical reaction can occur either in the phases or at the interface. It is often advantageous for all drops to have the same homogeneous chemical composition during the reaction process in order to maintain control of reaction rate and product quality. This requirement presents few problems if a batch process is used and all of the reaction ingredients are in the dispersion throughout the reaction process. In some processes, the addition of reaction ingredients is required during the process so that a batch process is replaced by a semi-batch operation. If the additional material is required to mix uniformly with the drop phase then it becomes necessary for drop coalescence and breakage to occur fairly readily. This may be achieved without difficulty if drop stabilisers are absent. However, it is sometimes necessary for drop stabilisers to be present as in suspension polymerisation because drop stability is 
required in the final product. Drop coalescence may then be hindered and feed material to a semi-batch reactor may not mix readily with drops of the dispersed phase.

In suspension polymerisation, monomer is suspended, as liquid droplets in a continuous water phase by the mean of a strong mechanical agitation and the presence of a suspending agent. In the system, continuous break-up and coalescence of the droplets occur simultaneously because of the turbulent conditions that exist. The stability of the formed droplets and the drop size in the system depend on a number of parameters. Among these parameters, the type and concentration of the suspending agent play an important role in the tendency of droplets to coalesce. As the suspension polymerisation proceeds, the viscosity of a monomer-polymer droplet increases with conversion. Hence, the physical behavior of the droplets is not the same during the process. When dispersible material is added to existing stabilised drops, a new material and existing drops can remain segregated for significant amounts of time (Zerfa, M. and Brooks, B.W. (1996a)).

With exothermic reaction processes, such as free-radical suspension polymerisation, some of the material in stabilised drops vaporises and is returned to the reactor after condensation. Refluxing the monomer is also done intentionally in order to remove the heat of polymerisation. The subsequent mixing between drops of condensate which are initially free of stabiliser and the stabilised drops can be slow. In copolymerisation where the ratio of monomers in the reflux is different from the ratio of monomers in the stabilised drop, the effect is more important. For semi-batch copolymerisation, controlled addition of comonomer ensures that the instantaneous polymer composition remains constant. As the viscosity of the droplets increases with time in the suspension polymerisation, the mixing rate of the new drops and stabilised drops may also affected. The incomplete mixing of the droplet will affect the properties of the polymer products.

\subsection{Objectives}

The objectives of the present research project are to investigate the behavior of drop mixing when new material is added to the liquid-liquid dispersion. This project 
is concerned primarily with suspension polymerisation. Viscosity is expected to be a major variable that will affect the drop mixing in the dispersion.

This work continues from the work performed by Zerfa, M. (1994) and, Zerfa, M and Brooks, B.W., (1996a) on the study of drop mixing in vinyl chloride polymerisation. In the study they found that pre-mixing time, stirring speed, type and concentration of surfactant affect the drop mixing rate between stabilised and unstabilised drops. Therefore, in this new work it was proposed to concentrate on the effect of the dispersed viscosity on the drop mixing in this type of dispersion. The viscosity is very important in the polymerization process since the viscosity increases as the polymerisation proceed.

It is intended to study the following aspects of the drop mixing in suspension polymerisation:

\section{A. Drop mixing}

A.1 The effect of viscosity on the drop size and drop size distribution of the dispersed phase before mixing with new material. The steady state of dispersion where the rate of coalescence and breakage are the same is determined.

A.2 Drop mixing between the new identical material with the pre-existing drops that are stabilised with a surfactant. The major parameter studied is the viscosity of the dispersed phase. Other parameter studied is stirring speed.

A.3 Drop mixing between two pre-existing drops that are stabilised with surfactant. The work is divided in two categories. First is the mixing of drops of two identical viscosities and second with the mixing of drops of two different viscosities.

A.4 The effects of type and concentration of surfactants.

B Drop Mixing Model 
B.1 Comparison between model developed in the literature and the results from these experiments. These model studies only focus on the coalescence between the drops of new material and the existing old drops.

\subsection{THESIS ORGANISATION}

The literature survey is presented in Chapter 2. It covers the liquid-liquid dispersion with major emphasis on the analysis of drop size and drop size distribution. Breakage and coalescence rate processes are also discussed with more detail description on coalescence process. Then the interfacial phenomena and surfactant system are presented. Lastly polymerisation processes are presented with detailed description of suspension polymerisation.

Chapter 3 explains the experimental apparatus, material and procedure that were involved in this work. Certain detailed explanation of the analysis is presented in the appendix $\mathrm{A}$ and $\mathrm{B}$.

Chapter 4 presents the results and the discussion of the work. They are presented in three parts. The first part is the preliminary work, second part is the experiments of adding batches of new material to existing old drops and the third part is the mixing of two dispersions. Some of the results are presented in graphs, while results in table form are presented in the appendix $C$.

Chapter 5 shows the work of comparison between experimental results and a literature model. The comparison is focused on the coalescence between the added new drops and the existing old drops.

Chapter 6 presents the conclusions of the work and also recommendations for further studies.

Lastly, chapter 7 lists the bibliography and references used in this work. 


\section{CHAPTER 2}

\section{LITERATURE REVIEW}

\subsection{Liquid-liquid dispersion}

Liquid-liquid dispersions have very important engineering applications and they are commonly used in reaction and separation processes. The increase of surface area due to the dispersion facilitates mass and heat transfer in the system. Therefore the understanding of the behavior of liquid-liquid dispersion is very important in their applications.

When two immiscible liquids are brought into contact in an agitated vessel, a dispersion of one phase into the other is formed. The drops are subject to shear stresses and to turbulent velocity and pressure variations along their surfaces. These processes cause the drops to deform and they may break into smaller parts. At the same time, drops are also colliding with each other, and if they remain together for enough time, the drops may coalesce. If the agitation continues over a sufficiently long time, a local dynamic equilibrium is established between break-up and coalescence (Valentas, K.H. and Amundson, N. R. (1966); Coulaloglou, C.A. and Tavlarides, L.L., (1977) and Narsimham, G. et al, (1979)). At this equilibrium, breakage and coalescence occur at the same rate so that prolonging the agitation does little to change average drop size and drop size distribution. The breakage and 
coalescence rates will depend on such variables as the geometry of the vessel and agitator, the energy input per unit mass, dispersed phase fraction and residence time (Shinnar, R. (1961); Miller R.S. et al (1963); Coulaloglou, C.A.and Tavlarides, L.L. (1977); Hong, P.O. and Lee, J.M. (1983) and Skelland, A.H.P. and Kennel, J.S. (1990)), as well as physical properties of the two phases. Among the physical properties that influence the coalescence and breakage rates are viscosities, densities and the interfacial properties (Vanderveen, (1960); Stamatoudis, M. and Tavlarides, L.L. (1985), Calabrese R.V. et al (1986); Wright, H. and Ramkrishna, D. (1994), Konno, M. et al (1982), Zerfa, M. and Brooks, B.W. (1996a).

In the absence of any surfactant, the coalescence efficiency, which is defined as the fraction of collisions resulting in coalescence, is close to unity (Valentas, K.H. et. al., 1966). The use of surfactant in the liquid-liquid dispersion may prevent coalescence as the surfactant is adsorbed on the surface of the drops. This process will enhance the drop stability by decreasing the interfacial tension. Suspension and emulsion polymerisation is a process that involves using surfactant in liquid-liquid dispersion operation. This review is restricted mainly to liquid-liquid dispersion related to suspension polymerisation. The major aim in suspension polymerisation is the formation of an as uniform as possible dispersion of monomer/polymer droplets in the continuous phase. Even though suspension polymerisation has been studied for a long time, the studies on breakage and coalescence phenomena are lacking.

The theoretical works of liquid-liquid dispersion have been an active field of study since the 1940s. A variety of experimental approaches have been used to investigate the liquid-liquid dispersion behavior. The most widely studied liquid-liquid dispersion system is carried out in batch agitated vessels. Kolmogorov A.N. (1941) began the theoretical work on liquid-liquid dispersion when he developed the theory of local isotropy. Later other workers extensively reviewed this theory and used it to study the process of breakage and coalescence.

\subsubsection{Effect of dispersed phase viscosity}

Dispersed phase viscosity is one of major variables that affect the behavior of 
liquid-liquid dispersion. In the suspension polymerisation process, the viscosity of dispersed phase is increasing during the process as the conversion increases. The increasing viscosity of the suspended droplets as polymerisation proceeds makes the quantitative analysis of suspension poymerisation a complex problem. Most earlier studies have been limited to a non-viscous dispersed phase which was limited to surface force stabilised dispersions.

The force associated with the continuous-phase turbulence makes the dispersed phase drops distort and break into smaller drops. This force is balanced by the surface force due to interfacial tension and the force associated with the viscosity of the drop. If the viscous force is large compare with the interfacial force, drop stability will be governed by the drop's viscosity. Hinze, J.O.(1955) has established a basis for data correlation when both surface and dispersed phase viscous forces contribute to drop stability.

Calabrese et al. (1986a) has examined the extent to which dispersed-phase viscosity influences the equilibrium mean drop size and drop size distribution at constant interfacial tension. The study was however limited to the dilute suspensions for which coalescence is negligible. Five different grades of silicone oils were dispersed in distilled water and these oils exhibit constant interfacial tension with water. The results of the study concluded that at constant conditions of agitation, the equilibrium drop size distribution broadens considerably as dispersed-phase viscosity increases. The ratio of sauter mean diameter, $d_{32}$ to maximum stable drop size, $d_{\max }$ did decrease to some extent as viscosity of the dispersed-phase increased.

In another study by Wang, C.Y.and Calabrese R.V., (1986), the relative contribution of interfacial tension and viscosity to drop stability were determined. In this study, nine viscosity grades silicone oils were dispersed in distilled water, methanol and three aqueous methanol solutions. The study shows that at lower viscosities of dispersed phase, $d_{32}$ increases slowly with the viscosity. However at higher viscosities the increase is more rapid. It was also found that at constant conditions of agitation, the relative influence of interfacial tension decreases as dispersed-phase viscosity increases. 
Kuriyama et al. (1996) has carried out dispersion experiments under conditions where the coalescence and break up of drops coexist. The dispersed phases were also silicone oils with different viscosities. It was found that at relatively low drop viscosity, the drop sizes substantially reach a constant value through agitation within about one hour. However with high drop viscosity, the drop size reduced even after a lapse of one hour. It was also found that the final drop size of dispersed phase of higher viscosity is higher. This study also confirmed that mean drop size (sauter mean) is always proportional to the maximum drop size.

If the dispersed phase of the dispersion is a polymer, the elastic properties must be taken into account. Arai, K. et al. (1977) have derived a correlation for drop break-up from a Voigt model to represent the elastic properties. The validity of the correlation was confirmed experimentally over a wide range of dispersed-phase viscosity. Konno, M. et al. (1982) has studied the coalescence of droplets in suspension polymerisation of styrene, they found that the sauter mean diameter increases as the polymer viscosity increases. They also concluded that stabiliser does not effectively prevent the coalescence of the dispersed drops of larger than $d_{\max }$.

The first attempt to include the viscosity effects in a more realistic way was undertaken by Alvarez, J.M. et al (1994). Their study includes the breakage and coalescence rates and will be discussed in detail in section 2.5 .

\subsubsection{Effect of surfactant}

Much of the work done on liquid-liquid dispersion has been on a noncoalescing system or with low dispersed phase ratio. Therefore most models and correlations currently available are based on this system which does not require a surfactant. However, for economic and efficiency reasons, most industries operate with the highest possible dispersed phase ratio. For some dispersion process, some surfactants are added to obtain stable suspensions as in the case of suspension polymerisation process. The surfactant adsorbs on the monomer/water interface and prevents the drops from coalescing. 
Several researchers (Church, J.M. and Shinnar, R., (1961); Hartland, S. (1968); Bowankar, R.P., (1986) and Konno, M. et al., (1993)) have used a surfactant in liquidliquid dispersion, they found that the average drop size is decreased when the concentration of surfactant is increased. Borwankar, R.P. et al., (1986) have studied the drop dispersion for suspension polymerisation and they determined the critical surface coverage of stabiliser necessary to inhibit coalescence and correlated the sauter mean diameter to the extent of agitation under turbulent conditions. The study by Chatzi, E.G. et al. (1991) has determined the minimum time required for a system to reach a steady state and the effects of stabiliser concentration, temperature and agitation speed on the average drop diameters.

Zerfa, M. and Brooks B.W. (1996a) have studied the effect of the suspending agent (surfactant) concentration on the size of the drops in vinyl chloride polymerisation. It was found that an increase in stabilizer concentration narrowed the size distribution. At low concentration, the drops were not protected well enough to prevent their coalescence. It was also found that at low stabilizer concentration, a sharp decrease in drop diameter was observed when concentrations of stabilizer were increased. However, there was also a critical stabilizer concentration above which the drop sizes remain steady.

\subsection{Turbulent mixing theory}

In liquid-liquid dispersion, the drops of the dispersed phase are continuously broken and coalesced at a rate depending on the local hydrodynamic characteristics of the flow field and the physical properties of the system. Under turbulence conditions, the breakage occurs due to the turbulence velocity and pressure variations along the surface of the drops (Hinze, J.O. 1955; Shinnar, R. 1961). This process deforms the drops and may break them into smaller droplets if the dynamic forces exceed the interfacial tension forces. Meanwhile, the coalescence occurs due to collisions of drops in the turbulent field, some of the collisions resulting in coalescence. The coalescence is accomplished through the draining and rupture of the film of the continuous phase between colliding droplets in the turbulent field (Howarth, W.J, 
1964). Therefore, in order to understand the breakage and coalescence processes, it is necessary to understand the turbulence conditions. The turbulence is considered to be a somewhat random flow of eddies superimposed on the overall average flow. The turbulence generated throughout the vessel is assumed isotropic. Larger eddies transfer their kinetic energy to smaller eddies, until energy is transferred to the smallest eddies. The large eddies are influenced by the vessel's boundary. Coulaloglou, C.A. and Tavlarides L.L, (1976) proposed that the size of large eddies is restricted to that of the impeller diameter. The energy transfer from larger to smaller eddies is assumed to take place without energy loss. Once the kinetic energy reaches the smallest eddies, it is dissipated as heat to overcome the viscous forces. The smallest eddies are statistically independent of each other and the small-scale velocity fluctuations are determined entirely by the local rate of energy dissipation per unit mass of fluid $(\varepsilon)$ and the kinematic viscosity $(v)$.

The Kolmogoroff length and velocity scales (Kolmogorov A.N., 1941) are defined as

$$
\begin{aligned}
& \eta=\left(\frac{v^{3}}{\varepsilon}\right)^{1 / 4} \\
& \gamma=(v \varepsilon)^{1 / 4}
\end{aligned}
$$

For local isotropy to exist, the macroscale of turbulence, $L$ of the energy containing eddies must be large compared to the microscale of turbulent $\eta(L>>\eta)$. The macroscale of turbulence, $\mathrm{L}$, can be approximated by the width of the fluid ejected by the agitator, i.e., the width of the impeller blade (Coulaloglou, C.A. and Tavlarides L.L (1976)). For the condition of local isotropy to be satisfied, the droplet diameters $d$ are much smaller than the macroscale of turbulence $L(d<<L)$, and much larger than the microscale of turbulence $\eta(d>>\eta)$. This range $(L>>d>>\eta)$ is also called initial subrange of turbulent, where the energy spectrum is independent of the kinematic viscosity and solely determine by the rate of energy dissipation. For droplets of diameter $d>>$, the viscous forces may be neglected in comparison with the inertial forces (turbulent pressure fluctuation), while for $d<<\eta$ viscous forces will dominate. 
Generally, it is assumed that for Reynolds numbers greater than 10,000 the flow field may be considered locally isotropic (Rushton, J.H. et al 1950). The Reynolds number is defined as:

$$
R e=\frac{N D^{2}}{v}
$$

where:

$$
\begin{aligned}
& N \text { is the impeller speed } \\
& D \text { is the impeller diameter }
\end{aligned}
$$

For local isotropy of turbulent flow, the mean square of the relative velocity, $u^{2}\left(d_{x}\right)$ between two points separated by a distance $d_{x}$, is given by Hinze, P.O. (1959)

$$
u^{2}\left(d_{x}\right)=k_{v}\left(\varepsilon d_{x}\right)^{2 / 3}
$$

where $k_{v}$ is a proportionality constant

\subsection{Physical properties of liquid-liquid dispersion}

When two immiscible liquid are mixed, there is a formation of a continuous phase and dispersed phase. Physical properties such as density and viscosity for both phases and the overall properties of the mixture are very important to determine the behavior of the liquid-liquid dispersion.

The average density of the two-phase dispersion is determined as a weighted average of the corresponding values of the dispersed phase and continuous phase. (Bouyatiotis, B.A. and Thornton, J.D. (1967)).

$$
\rho_{m}=\phi \rho_{d}+(1-\phi) \rho_{c}
$$

where 


$$
\begin{aligned}
& \rho_{m}=\text { density of mixture } \\
& \rho_{d}=\text { density of the dispersed phase } \\
& \rho_{c}=\text { density of the continuous phase } \\
& \phi=\text { dispersed phase ratio }
\end{aligned}
$$

For the viscosity of the liquid dispersion, it can be calculated by the following equation proposed by Vermeulen, T. et al. (1955)

$$
\mu_{m}=\frac{\mu_{c}}{1-\phi}\left(1+\frac{1.5 \mu_{d} \phi}{\mu_{d}+\mu_{c}}\right)
$$

where

$$
\begin{aligned}
& \mu_{m}=\text { viscosity of mixture } \\
& \mu_{d}=\quad \text { viscosity of dispersed phase } \\
& \mu_{c}=\quad \text { viscosity of continuous phase }
\end{aligned}
$$

\subsection{Drop Size Correlation}

Drop size and drop size distributions are the major important characteristics in liquid-liquid dispersion. The physical and chemical phenomena taking place in an agitated vessel largely depend on the size of the dispersed droplet. The drop size may be determined by using the arithmetic mean diameter $\left(d_{m}\right)$ which is obtained from the equation:

$$
d_{m}=\frac{\sum N_{i} d_{i}}{\sum N_{i}}
$$

or from the sauter mean diameter (volume to surface ratio) which is obtained from the equation:

$$
d_{32}=\frac{\sum N_{i} d_{i}{ }^{3}}{\sum N_{i} d_{i}{ }^{2}}
$$


where $N_{i}$ is the frequency of the drops of diameter size $d_{i}$.

Sauter mean diameter is extensively used to characterise the drop size properties in liquid-liquid dispersion because it links the area of dispersed phase to its volume.

The interfacial area per unit volume $A$ is related to the sauter mean diameter $\left(d_{32}\right)$ and the volume fraction of the dispersed phase $\phi$ through the equation:

$$
A=\frac{6 \phi}{d_{32}}
$$

This interfacial area per unit volume determines the transfer rate of energy, mass and chemical reaction in dispersions.

As the drops in the liquid-liquid dispersion are not uniform, investigators usually assume that there is a maximum drop diameter $\left(d_{\max }\right)$ above which stable drops do not exist (drop breakage control) and a minimum drop diameter $\left(d_{\min }\right)$ below which there are no stable drops (coalescence control).

\subsubsection{Maximum Stable Drop Size}

According to Hinze, J.O. (1955) and Shinnar, R. and Church, J.M., (1960) under noncoalescence conditions, the largest drop size $\left(d_{\text {max }}\right)$ is determined by the ratio of the kinetic energy of an oscillating drop due to turbulent fluctuation, $E_{v}$ and the energy due to the interfacial tension, $E_{s}$, where:

$$
\begin{aligned}
& E_{v}=\rho_{c} u^{2}\left(d_{x}\right) d_{x}^{3} \\
& E_{s}=\sigma d_{x}^{2}
\end{aligned}
$$

where $\sigma=$ Interfacial tension between two immiscible liquid 
Hence the Weber number which is defined as the ratio of the kinetic energy to the surface energy has a critical value for break-up $W e_{c r i t}$ of

$$
W e_{\text {crit }}=\frac{E_{v}}{E_{s}}=\frac{\rho_{c} u^{2}\left(d_{x}\right) d_{x}}{\sigma}
$$

Introducing equation [2.4], then

$$
W e_{b}=\frac{k_{v} \rho_{c} \varepsilon^{2 / 3} d_{x}^{5 / 3}}{\sigma}=\text { constant }
$$

Therefore for $d>>\eta$, the following equation for the largest drop sizes, $d_{\text {max }}$ is

$$
d_{\max }=C_{1}\left(\frac{\sigma}{\rho_{c}}\right)^{3 / 5} \varepsilon^{-2 / 5}
$$

where $C_{l}$ is a constant

According to Rushton J.H. et al (1950), for a high Reynolds number, the energy input of mixing by the impeller per unit mass of liquid in the vessel is independent of liquid properties and is a function of the geometrical design of the agitator and its speed, related as

$$
\varepsilon=C_{2} N^{3} D^{2}
$$

where

$C_{2}=$ a constant that depends on the geometry of the vessel and on the agitator

Therefore the following equation is obtained for $d_{\max }$

$$
d_{\max }=C_{3}\left(\frac{\sigma}{\rho_{c}}\right)^{3 / 5} N^{-6 / 5} D^{-4 / 5}
$$

The expression can be simplified further as follows 


$$
d_{\text {max }}=C_{3} D\left(\frac{\rho_{c} N^{2} D^{3}}{\sigma}\right)^{-3 / 5}=C_{3} D(W e)^{-0.6}
$$

and

$$
\frac{d_{\operatorname{mix}}}{D}=C_{3}(W e)^{-0.6}
$$

where $C_{3}$ is a constant

By assuming a linear relationship between $d_{\max }$ and $d_{32}$ (Sprow, F.B. (1967) and Coulaloglou, C.A. and Tavlarides, L.L. (1976)) used:

$$
d_{32}=C_{4} d_{\max }
$$

therefore:

$$
\frac{d_{32}}{D}=C_{3} C_{4}(W e)^{-0.6}=C_{5}(W e)^{-0.6}
$$

where $C_{4}$ and $C_{5}$ are constants

As the dispersed-phase fraction $\phi$ increases, equation [2.20] needs to be modified to account for the increase in droplet size due to the damping of turbulence by the droplets. The modified correlation is usually in the form of (Yuan, H.G. et al., 1991):

$$
\frac{d_{32}}{D}=C_{6} f(\phi)(W e)^{-0.6}
$$

where the correlation factor $f(\phi)$ expressed empirically by the linear relation as $(1+$ $\left.C_{7} \phi\right)$.

$C_{6}$ and $C_{7}$ are constants.

Equation [2.21] gives good results only when the drop diameter $d$ is much larger than the Kolmogoroff length $\eta$ and the dispersed-phase viscosity $\mu_{d}$ is sufficiently small. The equation become inappropriate as the larger values of viscosity play an important 
part in resisting the deformation of the drops. Hinze, J.O. (1955) has analysed the effect of dispersed-phase viscosity on $W e_{c r i t}$ and he proposed an independent dimensionless group, a viscosity number $N_{v i}$ defined as

$$
N_{v i}=\frac{\mu_{d}}{\sqrt{\rho_{d} \sigma D}}
$$

He postulated that a drop would break at a critical Weber number, which increases as the value of $N_{v i}$ increases, such that

$$
W e_{c r i t}=C_{8}\left[1+f\left(N_{v i}\right)\right]
$$

Where the function $f\left(N_{v i}\right)$ decreases to zero for low $\mu_{d}$ so that $C_{8}$ represents the critical Weber number for non-viscous drops.

Arai, K. et al. (1977) redefined the viscosity number when they derived an expression from Voigt model as:

$$
N_{v i}=\frac{\mu_{d} \varepsilon^{1 / 3} d^{1 / 3}}{\sigma}
$$

and they obtained the expression for maximum stable drop size as:

$$
\frac{\rho_{c} \varepsilon^{2 / 3} d_{\max }^{5 / 3}}{\sigma\left(1+f\left(N_{v i}\right)\right)}=\text { constant }
$$

Calabrese, R.V. et al. (1986a) also applied the viscosity group number in the droplet size correlations. However, the range of viscosity they used was very low (up to 10 Pa.s). They considered the dispersed drops as having cohesive energy composed of surface and viscous energy. Therefore a droplet is opposed by distruptive energy due to continuous-phase turbulence and is stabilised by cohesive energy due to interfacial tension and dispersed-phase viscosity. The correlation was written in general form as:

$$
\frac{d_{32}}{d_{o}}=\left[1+C_{9} N_{v i}\right]^{3 / 5}
$$


where

$$
\begin{aligned}
N_{v i} & =\frac{\left(\rho_{c} / \rho_{d}\right)^{1 / 2} \bar{\varepsilon}^{-1 / 3} d_{32}}{\sigma} \\
C_{9} & =\text { constant } \\
d_{o} & =\text { the sauter mean diameter for inviscid dispersed phase }
\end{aligned}
$$

\subsubsection{Minimum Stable Drop Size}

Drop coalescence is significant in a liquid-liquid dispersion when the volume fraction of dispersed phase is sufficiently large. The minimum droplet size $\left(d_{\min }\right)$ depends on the ratio of the turbulent fluctuation energy $\left(E_{v}\right)$ to the adhesion energy $\left(E_{a}\right)$, which tends to hold two colliding droplets together (Shinnar, R. and Church, J.M., 1960).

The adhesion energy is defined as

$$
E_{a}=d A\left(h_{o}\right)
$$

where $A\left(h_{o}\right)$ is a constant $\left(h_{o}\right.$ being the smallest distance between two drops of equal diameter $d$ ).

When $E_{a}>E_{w}$ coalescence of two droplets occur and when coalescence is dominated in the liquid dispersion $d_{\min }$ is therefore determined by

$$
\frac{E_{v}}{E_{a}}=\frac{\rho_{c} u^{2}\left(d_{x}\right) d_{x}{ }^{3}}{A\left(h_{o}\right) d}=\frac{\rho_{c} u^{2}\left(d_{x}\right) d_{x}{ }^{2}}{A\left(h_{o}\right)}=\text { constant }
$$

For local isotropy and $d>>\eta$,

$$
u^{2}\left(d_{x}\right)=k_{v}(\varepsilon d)^{2 / 3}
$$


and

$$
\varepsilon=C_{2} N^{3} D^{2}
$$

Therefore, after substitution the equation can be written as

$$
\begin{aligned}
& \frac{C_{10} \rho_{c} \varepsilon^{2 / 3} d_{x}^{8 / 3}}{A\left(h_{o}\right)}=\frac{C_{10} \rho_{c} N^{2} D^{4 / 3} d_{x}^{8 / 3}}{A\left(h_{o}\right)}=C_{11} \rho_{c} N^{2} D^{4 / 3} d_{x}^{8 / 3}=\text { constant } \\
& \therefore d_{\text {min }}=C_{12} \rho_{c}^{3 / 8} N^{-3 / 4} D^{-1 / 2}
\end{aligned}
$$

and

$$
\frac{d_{\min }}{D}=C_{12}(W e)^{-3 / 8}
$$

If the values of $d_{32}$ are considered to be approximately proportional to $d_{\min }$, then

$$
\frac{d_{32}}{D}=C_{13}(W e)^{-3 / 8}
$$

$C_{10}, C_{11}, C_{12}$ and $C_{13}$ are constants

\subsubsection{Simultaneous break-up and coalescence}

The drop size and size distribution in an agitated liquid-liquid dispersion, in reality, are determined by both break-up and coalescence, as they occur simultaneously. Therefore the mean droplet size is determined by the dynamic equilibrium between the break-up and coalescence of droplets and for a non-viscous dispersion and $d>>\eta$ the correlation is:

$$
C_{5}(W e)^{-3 / 5} \leq \frac{d_{32}}{D} \leq C_{13}(W e)^{-3 / 8}
$$




\subsubsection{Drop Size Distribution}

The mean drop size cannot fully characterize a dispersion because the same mean drop diameter can be obtained for dispersions with difference size distributions. Therefore to describe a dispersion, both the mean drop size and the drop size distribution are needed. A lot of work has been done to characterise the drop size distribution of dispersions. The normal distribution function describes the drop size distribution. Chen, H.T. and Middleman, S. (1967) used a normal distribution function, normalised with respect to $d_{32}$, to represent the drop size distribution of a xylene/water dispersion. The equation was written as a volumetric probability function,

$$
f_{v}\left(\frac{d}{d_{32}}\right)=\frac{1}{0.23 \sqrt{\pi}} \exp \left[-9.2\left(\frac{d}{d_{32}}-1.06\right)^{2}\right]
$$

Brown, D.E. and Pitt, K. (1972) used an equation similar to the last equation to represent the drop size distribution of a kerosene/water dispersion,

$$
f_{\nu}\left(\frac{d}{d_{32}}\right)=\frac{1}{0.20 \sqrt{\pi}} \exp \left[-12.5\left(\frac{d}{d_{32}}-1.07\right)^{2}\right]
$$

Calabrese, R.V et al (1986b) also have derived the correlation for drop size distribution for low dispersed phase fraction and from low to moderate viscosity. The correlation was also written as volume probability function as follows:

$$
f_{v}\left(\frac{d}{d_{32}}\right)=\frac{1}{0.23 \sqrt{2 \pi}} \exp \left[-9.1\left(\frac{d}{d_{32}}-1.07\right)^{2}\right]
$$

The dynamic behavior of a dispersion in an agitated tank and its drop size distribution are determined by the breakage and coalescence processes of the dispersed drops. If the dynamic character of breakage and coalescence is formulated, it is possible to predict the drop size distribution using mathematical modeling. Several models have 
been developed by researchers in order to describe the behavior of the liquid-liquid dispersions in agitated vessels. A population balance approach has a significant advantage, as it is possible to include in the model details relative to breakage and coalescence processes in terms of physical parameters and operating conditions. It has been used extensively in the literature for the mathematical modeling of liquidliquid dispersion. The population balance concept was proposed by Hulbert, H.M.and Katz, S. (1964) and developed later by a number of investigators (Valentas, K.J. and Admunson, N.R. (1966); Ramkrishna, D. (1974); Bajpai, R.K. et al (1976), Coulaloglou, C.A. and Tavlarides, L.L. (1977); Sovova, H (1981); Stamatoudis, M. and Tavlarides, L.L., (1985); Chatzi, E.G. and Lee, J.M. (1987) and Tsouris, S. and Tavlarides, L.L. (1994)). In the population balance formulation, the dispersed phase is viewed as a group of droplets whose identities are being continuously destroyed and re-created by the dynamic processes of break-up and coalescence.

For batch liquid-liquid dispersion, the population balance equation has the following conservation equation,

$$
\text { [Birth rates of drops] }-[\text { Death rates of drops }]=[\text { Accumulation term }]
$$

The corresponding population balance equation can be derived as (Stamatoudis, $\mathbf{M}$. and Tavlarides, L.L., 1985):

$$
\int_{v}^{v_{\max }} g\left(v^{\prime}\right) v\left(v^{\prime}\right) \beta\left(v^{\prime}, v\right) d v^{\prime}+\int_{0}^{v / 2} \kappa\left(v-v^{\prime}, v^{\prime}\right) d v=g(v)+\int_{0}^{v_{\max }} \kappa\left(v, v^{\prime}\right) d v^{\prime}+\frac{d[N(t) A(v)]}{d t}
$$

where

$$
\begin{aligned}
g(v)= & \text { the number of drops of size } v \text { breaking per unit volume of } \\
& \text { dispersion per unit time. } \\
\kappa\left(v, v^{\prime}\right) d v^{\prime}= & \begin{array}{l}
\text { the number of pairs of drops of size } v \text { and } v^{\prime} \text { coalescing per unit } \\
\text { volume of dispersion per unit time. }
\end{array} \\
v\left(v^{\prime}\right)= & \text { the number of daughter particles resulting from a breakage of a } \\
& \text { drop of volume } v^{\prime} .
\end{aligned}
$$




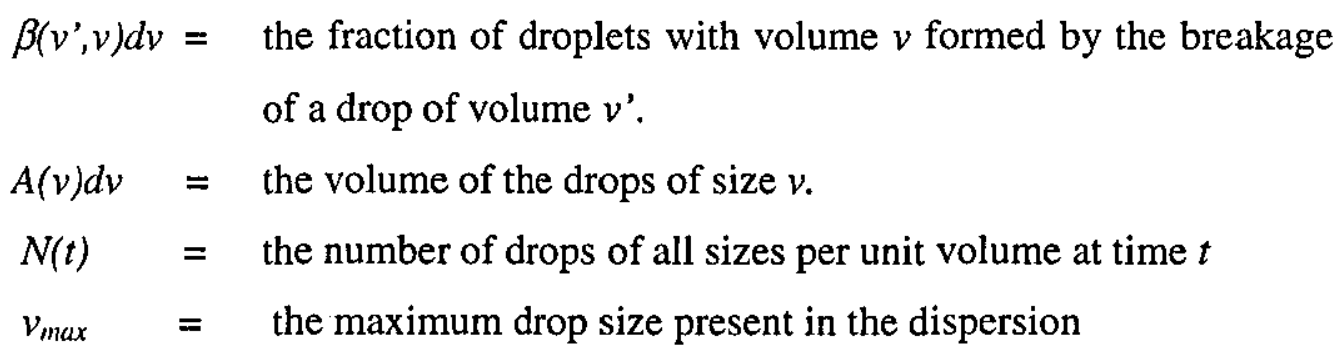

The terms on the left-hand side represent the contribution of drop volume $v$ by breakage of larger drops and by coalescence of smaller drops, respectively. The first two terms on right hand side represent the loss of drops of size $v$ due to breakage and to coalescence with other drops. The last term on the right hand side is the accumulation term. The drop breakage rate function $g(v)$ depends on the surface tension and on the hydrodynamic field outside drops. The drop coalescence rate $\kappa\left(v, v^{\prime}\right)$ depends on the turbulence condition and on the coalescence efficiency between colliding drops.

\subsection{Breakage and Coalescence Rate}

The evolution of the drop size distribution of the liquid dispersion in a stirred tank reactor is controlled by the breakage and coalescence processes. The interaction rates of the dispersed phase drops depend on the local hydrodynamic characteristics of the flow field and the physical properties of the system.

A lot of work has been done for the study of the break-up and coalescence in liquidliquid dispersion in the past. However, the main focus of these studies in on the effect of physical properties of the system or geometrical and operating conditions on the steady-state average drop size or drop size distribution. Furthermore, existing descriptions for the breakage and coalescence rate are not directly applicable in suspension polymerization because suspension viscoelasticity effects are not accounted for. Alvarez, J.M. et al. (1994) has derived a suitable expression for breakage and coalescence rate in suspension polymerisation. They derived the expression which account for the simultaneous presence of interfacial tension, drop viscoelasticity and water-film drainage. Recently Maggioris, D. et al (2000) used the model to predict reasonably well the experimentally observed inhomogeneities of the 
drop size distribution as well as the evolution of drop size distribution in Vinyl chloride suspension polymerisation.

The coalescence and breakage rate can be represented in terms of frequency and Maxwellian efficiency expressions.

$$
\begin{aligned}
& g(x)=\omega_{b}(x) e^{-\lambda_{b}(x)} \\
& \kappa(x, y)=\omega_{c}(x, y) e-^{\lambda_{r}(x, y)}
\end{aligned}
$$

where

$$
\begin{aligned}
& g(x)=\text { breakage rate of a drop having volume } \mathrm{x} \\
& \kappa(x, y)=\text { coalescence rate of two drop having volume } \mathrm{x} \text { and } \mathrm{y} \\
& \omega_{b}(x)=\text { eddy-drop collision frequency of a drop having volume } \mathrm{x} \\
& \omega_{c}(x, y)=\text { collision frequency of two drops having volumes } \mathrm{x} \text { and } \mathrm{y}
\end{aligned}
$$

\subsubsection{Breakage rate}

Considerable amount of work on drop break-up rate model has been proposed in the literature. Some of the models basically consider two mechanisms, first, the deformation of a drop and, second, the probability of break up of the deformed drops. Such a mechanistic model has been developed by Coulaloglou, C.A. and Tavlarides, L.L (1977) and Narsimhan, G. et al (1979). The model provides simple mathematical expressions for the drop breakage rate which includes the physical properties of the system, the geometry and the energy provided to the dispersion by agitation.

Coulaloglou, C.A. and Tavlarides, L.L (1977) derived a model for breakage by considering drop deformation and break-up under the influence of local pressure fluctuations and the time needed for a critically deformed drop to break-up in a locally isotropic field. The breakage frequency was derived as,

$$
g\left(d_{x}\right)=k_{1} \frac{\varepsilon^{1 / 3}}{(1+\phi) d_{x}{ }^{2 / 3}} \exp \left(-k_{2} \frac{\sigma(1+\phi) 2}{\rho d_{x} \varepsilon^{2 / 3} d_{x}{ }^{5 / 3}}\right)
$$


where $k_{1}$ and $k_{2}$ are proportionality constants

Tsouris and Tavlarides (1994) introduced a drop breakage function as the product of the drop-eddy collision frequency and breakage efficiency, which reflected the energetic of turbulent liquid-liquid dispersion.

$g\left(d_{x}\right)=0.118 D F(\phi) \varepsilon^{1 / 3} \int_{2 / d_{x}}^{\frac{2}{d_{c, \text { min }}}}\left(\frac{2}{k}+d_{x}\right)^{2} \times\left(8.2 k^{-2 / 3}+1.07 d_{x}^{2 / 3}\right)^{1 / 2} k^{2} \exp \left[-\frac{E_{c}}{k_{3} e}\right] d_{x} k n_{d}$

where

$D F(\phi)=$ turbulent damping factor

$$
=\left[1+2.5 \phi\left(\frac{\mu_{d}+0.4 \mu_{c}}{\mu_{d}+u_{c}}\right)\right]^{2}
$$

$d_{e}=$ eddy diameter

$k=$ eddy wavenumber

$E_{c}=$ energy required for breakage

$N_{d}=$ number of drops

$k_{3}=$ proportionality constant

The model proposed by Alvarez, J.M. et al (1994) assumes that breakage of a drop exposed to a turbulent flow field is a result of fluctuation with wavelengths equal to the drop diameter. Fluctuations with wavelengths that are smaller or larger than the drop diameter $d_{x}$ produce oscillatory or rigid-body motion of the drop, respectively, without resulting in a breakage event. Therefore, by assuming that only fluctuations with wavelengths $d_{x}$ can induce breakage of drops of diameter $d_{x}$, the frequency term can be written as,

$$
\omega_{b}=u\left(d_{x}\right) / d_{x}
$$


For drop size similar to eddy wavelengths in the local isotropy of turbulence, it follows from equation [2.4], that,

$$
\omega_{b}=k_{b} \frac{\varepsilon^{1 / 3}}{d_{x}^{2 / 3}}
$$

where

$$
k_{b}=k_{v}{ }^{1 / 2}
$$

According to the model, for droplet breakage to occur, the surface energy and viscoelastic resistance, which are important in suspension polymerisation, must be overcome. The breakage efficiency definition was based on the ratio of available to required energy for breakage and is given by,

$$
\lambda_{b}(x)=a_{b} \Omega\left(d_{x}\right)
$$

where

$$
\begin{aligned}
& \Omega\left(d_{x}\right)=\frac{6}{\operatorname{Re}\left(d_{x}\right)\left[1+\operatorname{Re}\left(d_{x}\right) V_{e}\left(d_{x}\right)\right]}+\frac{1}{W e\left(d_{x}\right)} \\
& a_{b}=\text { proportionality constant }
\end{aligned}
$$

The drop Reynold number, $\operatorname{Re}\left(d_{x}\right)$ and drop Weber number, $W e\left(d_{x}\right)$ are defined as

$$
\begin{aligned}
& \operatorname{Re}\left(d_{x}\right)=d_{x y} \frac{u\left(d_{x y}\right)}{v_{s}} \\
& \text { and } \\
& W e\left(d_{x}\right)=\frac{\rho_{s} d_{x} u^{2}\left(d_{x}\right)}{\sigma}
\end{aligned}
$$

Viscoelasticity is accounted for by the dimensionless magnitude $V_{e}$ and can be determined from the following equation:

$$
V_{e}=\Theta \exp \left(-\frac{1-\alpha}{2 Y_{o} \operatorname{Re}}\right)-\frac{1}{12}
$$


where

$$
\begin{aligned}
& \alpha=\sqrt{1-48 Y_{o}} \\
& \Theta=\frac{Y_{o}}{\alpha(1-\alpha)}\left[1+\alpha-\frac{(1-\alpha)^{2}}{1+\alpha}\right] \exp \left(-\frac{1-\alpha}{2 Y_{o} \operatorname{Re}}\right)
\end{aligned}
$$

and

$$
Y_{o}=\frac{\mu_{d}^{2}}{\rho_{d} E_{d} d_{x y}^{2}}
$$

where

$$
E_{d}=\text { dispersed phase elasticity modulus }
$$

The dispersed phase elasticity modulus, can be defined as a function of polymer elasticity modulus $E_{p}$ and conversion, $\chi$, as

$$
E_{d}=\chi E_{p}
$$

\subsubsection{Coalescence Rate}

The coalescence process is more complex than the break-up process since it involves not only the approach of two drops, but also the drainage and rupture of the intervening liquid film, in which the physical properties of the fluids and interfaces play an important role. Most of the investigators have viewed the approach of drops and their subsequent coalescence in two steps, collision and coalescence. The collision is when the drops approach each other while the coalescence is where the drainage and the rupture of intervening film occur. The coalescence of the two drops is accomplished through the draining and rupture of the film of the continuous phase. During the collision between two drops, they may be rebound, cohere or coalesce. The coalescence drops may then broken up by the action of turbulent force.

In the turbulent mixing, drops caught in an eddy experience a squeezing force exerted by the eddy on them. When two drops approaching each other, they maintain their spherical shape, and as the distance separating them becomes smaller their approach velocity decreases rapidly while the pressure in the tip region increases. At some stage, because of the pressure increase, the drops begin to flatten. 
Therefore the shape changes from perfectly spherical to a disc at the tip. The drainage rates are significantly affected by the presence or absence of the disc. The coalescence time decreases with the decreasing size of the disc because of the shorter distance over which the film needs to be drained. Apart from the presence of the disc, the main parameters determining the rate of drainage are the mobility of the interface and the rupture thickness. With higher interfacial mobility, the velocity of the flow at the interface increases and therefore also the drainage rate increases. Barber A.D. and Hartland S.(1976) suggest that for a surface saturated with surfactant, the interface is not likely to be completely immobile but will move at a rate determined by its surface viscosity and a rate of liquid flow adjacent to it.

A number of coalescence models have been reported in the literature. The coalescence rate, required for the solution of the population balance equations, is defined as the product of collision frequency and coalescence efficiency. Various expressions for collision frequency have been proposed (Howarth, W.J.(1964); Abrahamson, J. (1975); Coulaloglou, C.A. and Tavlarides, L.L (1977) and Das, P.K. et al.(1987). By assuming that the mechanism of collision in a locally isotropic flow field is analogous to collisions between molecules as in the kinetic theory of gases, the frequency between two drops with volume $\mathrm{x}$ and $\mathrm{y}$ can be expressed as (Coulaloglou, C.A. and Tavlarides, L.L. (1977),

$$
\omega_{c}=k_{c}\left(d_{x}{ }^{2}+d_{y}{ }^{2}\right) \sqrt{u^{2}\left(d_{x}\right)+u^{2}\left(d_{y}\right)}
$$

where

$k_{c}$ is a proportionality constant

after substituting by using equation (2.4), the last expression can be written as

$$
\omega_{c}=k_{c} \varepsilon^{1 / 3}\left(d_{x}{ }^{2}+d_{y}{ }^{2}\right) \sqrt{d_{x}^{2 / 3}+d_{y}^{2 / 3}}
$$

Shinnar, R (1961) considered that two drops are unlikely to coalesce immediately on collision, but may cohere together and be prevented from coalescing by a film of the continuous-phase liquid trapped between them. The coalescence occurred after the film separating the cohering drops drained or diffused away until collapsed. The 
cohesion of the drops was assumed to be due to attractive forces, but the nature of the forces was not discussed. However it was believed that the cohesive forces were dependent of drop diameter and the cohering drops could not be parted at a given intensity of turbulence if their size was smaller than a minimum value.

Howarth, W.J. (1964) proposed that immediate coalescence would occur when the velocity of approach along a line of centres at the instant of collision exceeds a critical value. From this he derived the coalescence efficiency as

$$
\lambda_{c}=\left(-\frac{3 w_{*}}{4 u^{2}}\right)
$$

where

$$
\begin{aligned}
& w_{*}=\text { critical approach velocity } \\
& u^{2}=\text { mean square turbulent velocity }
\end{aligned}
$$

Based on this equation, the frequency of coalescence of uniformly sized drops in a homogeneous isotropic turbulent flow dispersion of infinite extent was derived. The equation was tentatively confirmed by comparison with the experimental data produced Madden, A.J. and Daramell, G.L. (1962).

The above expression is independent of fluid properties as the model neglected the presence of the drainage and rupture of the intervening film. Coulaloglou, C.A. and Tavlarides, L.L (1977) considered film drainage and rupture in the expression of the coalescence efficiency. They suggested that for coalescence to occur, the time of contact between drops must exceed the time required for coalescence. The coalescence time was estimated as the time required for film drainage between drops and the contact time was estimated to be the time required for two drops of size $d_{x}$ and $d_{y}$ to stay together in a turbulent field. They derived the coalescence efficiency as

$$
\lambda_{c}=\left[\left(-\frac{k_{4} \mu_{c} \rho_{c} \varepsilon}{\sigma^{2}}\right)\left(\frac{d_{x}+d_{y}}{d_{x} d_{y}}\right)^{4}\right]
$$

where

$k_{4}=$ parameter that strongly depends on the critical thickness of film rupture 
There are also other models that predict the coalescence efficiency. However most of the models were independent of the system properties. Das, P.K. et al (1987) developed a white-noise model for drop coalescence which considered the film drainage between two colliding drops as a stochastic process driven by a suitably idealised random process for the fluctuating force applied on the drops.

A work by Kumar, S (1993) has put the system properties in the model. He defined the coalescence efficiency as the ratio of the range of angle resulting in coalescence to the total range of all possible approach angles. The study on the model of coalescence efficiency found that dispersed-phase viscosity is one of the parameters that influences the coalescence efficiency. Wright, H. and Ramkrishna, D. (1994) have studied the parameters that influence the drop coalescence rate on a dispersion of benzene/carbon tetrachloride in water. They found that the coalescence rate depended on dispersed-phase fraction, impeller speed and volumes of the coalescing droplets.

Tsouris, S. and Tavlarides, L.L. (1994) have also reviewed the model for the coalescence process. The expression that they derived for coalescence efficiency is given as

$$
\lambda_{c}=-\frac{6 \pi \mu_{c} k_{5} \zeta}{\rho_{c} \varepsilon^{2 . / 3}\left(d_{x}+d_{y}\right)^{2 / 3}} \frac{31.25 N D}{\left(U^{2} H\right)^{1 / 3}}
$$

where

$$
\begin{aligned}
& \zeta=1.872 \ln \left[\frac{h_{1}^{1 / 2}+1.378 q}{h_{2}^{1 / 2}+1.37 q}\right]+0.127 \ln \left[\frac{h_{1}^{1 / 2}+0.312 q}{h_{2}^{1 / 2}+1.378 q}\right] \\
& q=\frac{a^{1 / 2}}{\phi} \\
& a=\frac{r_{x} r_{y}}{r_{x}+r_{y}}
\end{aligned}
$$

and

$$
\begin{aligned}
& r=\text { drop radius } \\
& h_{1}=\text { film thickness at time } t_{1}
\end{aligned}
$$




$$
\begin{aligned}
& U=\text { Vessel diameter } \\
& H=\text { Vessel Height } \\
& k_{5}=\text { a constant }
\end{aligned}
$$

Liu, S. and Li, D. (1999) assumed that the contact time between drops is exponentially distributed and they defined a coalescence efficiency function as

$$
\lambda_{c}=0.0272 \frac{\mu_{c} \rho_{c}^{0.84} \varepsilon^{0.89} d_{x}^{3.11}}{\sigma^{1.38} \mathbf{B}^{0.46}}
$$

where

$$
\mathrm{B}=\text { London-van der Waals constant }
$$

For a suspension polymerisation process, the above expressions for coalescence efficiency may apply to the initial (low-viscosity) period but not to the sticky stage, where suspension viscoelasticity becomes important and plays a critical role in determining the stability. Instead of a time approach, they used an energy approach where fluctuation and deformation times will be implicitly accounted for. The coalescence efficiency function was obtained in terms of the ratios of required to available energy.

They derived the coalescence efficiency as follows:

$$
\lambda_{c}(x, y)=a_{c} \Omega\left(d_{x y}\right)-b_{c} / W e_{d}\left(d_{f}\right)
$$

The first part of the right hand side of the equation was contributed by viscoelasticity and surface tension effect, while the second part was contributed by film drainage effect. Below are the detailed description of the variables involved in the equation.

The parameter $d_{x y}$ is the mean length for deformation in coalescence interpenetration. The drop interpenetration is necessary for coalescence to occur. Ivanov, I.B. et al., (1985) described that when two drops collide, the smaller one has a larger energy content and it undergoes a larger deformation. The process of 
coalescence of two drops is shown in figure 2.1.

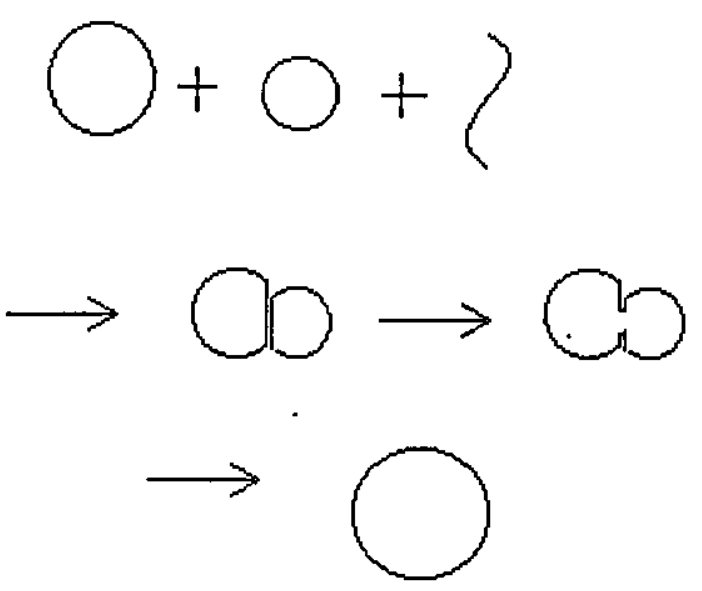

Figure 2.1: Schematic diagram of the process of generation of turbulence and coalescence of drops

The mean length for deformation was defined as,

$$
d_{x y}(x, y)=\left(d_{x}^{-1}+d_{y}^{-1}\right)
$$

The parameter $W e_{d}$ in equation (2.64) is drainage Weber number that contributed the drainage effects to the coalescence efficiency and is defined as,

$$
W e_{d}=\rho_{w} d_{f} v^{2}\left(d_{f}\right) / \sigma
$$

where

$$
\begin{aligned}
\rho_{w}= & \text { water density } \\
d_{f}= & \text { effective diameter for film drainage } \\
& =\left(d_{x}{ }^{3}+d_{y}{ }^{3}\right) / d_{x y}{ }^{2}
\end{aligned}
$$




\subsection{Previous Experimental Approach}

A variety of experimental approaches were used to measure the drop sizes in the mixing vessel. Some approaches measured the drop sizes directly in the mixing vessel (Borwankar, R.P. et al. (1986), Calabrese, R.V. et al. (1986) and Kuriyama, M. et al. (1996)). This method was used especially on drop mixing without using stabilizer. However for drop mixing with stabilizer, measurement outside the mixing vessel was widely used.

Chatzi, E.G. and Kiparissides, C. (1995) used a stainless steel tube to withdraw samples from the mixing vessel. The sample was directly introduced into a dilution vessel containing a predetermined quantity of concentrated PVA solution. It was noticed that measurements of the droplet-size distribution of different samples collected from the dilution vessel did not show any significant changes over a period of 20 minutes.

In the study by Zerfa, M. and Brooks B.W. (1996a), the sample was withdrawn into an optical cell, which was filled with the same concentration of PVA as in the mixing vessel. It was found that when a dispersion sample was left in the optical cell for 48 hours, the drop showed no change in sizes.

Laso, M. et al. (1987) also determined drop size by drawing a sample of the vessel contents into a capillary tube, photographing and returning it immediately to the vessel. In order to simulate the drop dispersion for suspension polymerization, many researchers used a model system. Borwankar, R.P. et al. (1986) chose the model system of $30 \%$ by volume 1,2-dichloroethane and $70 \%$ ethylbenzene to match the solubility parameter and density of vinyl chloride monomer. Chatzi, E.G. et al. (1991) however chose styrene in water for the experimental studies of the liquid dispersion. For the studies of different viscosity of dispersed phase, Arai, K. (1977) used a model system of polystyrene-0-xylene .

A variety of experimental techniques were used in the past to study the drop mixing. Among these techniques was a dye tracer. Zerfa, M. and Brooks, B.W. (1996b) have used this technique successfully in measuring drop coalescence process in suspension 
polymerization of vinyl chloride. It was found that the degree of coalescence was mainly controlled by the mixing time, the agitator speed, and the type and concentration of suspending agent. The rate of coalescence rises slowly with the mixing time, is roughly proportional to the agitator speed, and decreases sharply when the concentration of suspending agent is increased.

\subsection{Interfacial Phenomena}

The term interface indicates a boundary between any two immiscible phases. It is characterized by its interfacial free energy, which is the minimum amount of work required to create the interface. The interfacial free energy per unit area is the interfacial tension between two phases. It is the minimum amount of work required to create unit area of the interface.

In liquid-liquid dispersion, the interfacial area between the two different phases is greatly increased. This increase in surface area results in an increase in the interfacial free energy of the system. As a result, the system become highly unstable and will attempt to revert to the earlier state in order to reduce its interfacial free energy. In order to form a stable droplet in liquid-liquid dispersion, the interfacial free energy must be reduced without reducing the surface area. This is achieved by using a surfactant, which adsorbs at the liquid-liquid interface. The surfactant act to reduce the interfacial free energy and thus the interfacial tension.

A surfactant which adsorbs as an oriented interfacial film forms a mechanical, steric or electrical barrier around the dispersed droplets and prevents their coalescence (Rosen, M.J., 1989). The mechanical barrier prevents the coalescence of drops during collision, while the steric and electrical barriers inhibit the close approach of one drop to another. The total energy interaction energy between the droplets can be written as (Ottewill, R.H., 1967):

$$
\Delta G_{\text {total }}=\Delta G_{\text {electrostatic }}+\Delta G_{\text {van der waals }}+\Delta G_{\text {steric }}
$$

where : 
$\Delta G_{\text {electrostatic }}$ is due to electrostatic forces of repulsion

$\Delta G_{\text {van der wauls }}$ is an attraction due to van der waals forces

$\Delta G_{\text {steric }}$ is a repulsion force due to the steric effects

\subsubsection{Surfactant System}

The term surfactant is a contraction of surface active agent and refers to a class of chemical compounds known as amphiphiles. This amphipathic structure has a characteristic molecular structure consisting of a structural group that has very little attraction for the solvent, known as lyophobic group, together with a group that has strong attraction for the solvent, called the lyophilic group. The terms hydrophobic and hydrophilic are used if the liquid medium is aqueous. The hydrophobic group is usually a long-chain hydrocarbon residue and the hydrophilic group is an ionic or highly polar group.

The surfactants are classified depending on the nature of the hydrophilic group as:

1. Anionic

The surface-active part of the molecule carries a negative charge.

Example: Sodium Stearate $\mathrm{CH}_{3}\left(\mathrm{CH}_{2}\right)_{16} \mathrm{COONa}^{+}$

Sodium Lauryl Sulphate $\mathrm{CH}_{3}\left(\mathrm{CH}_{2}\right)_{11} \mathrm{SO}_{4} \mathrm{Na}^{+}$

2. Cationic

The surface-active part bears a positive charge.

Example: Dodecylamine hydrochloride $\mathrm{CH}_{3}\left(\mathrm{CH}_{2}\right)_{11} \mathrm{NH}_{3}{ }^{+} \mathrm{Cl}$

Hexadecyltrimethyl ammonium bromide $\mathrm{CH}_{3}\left(\mathrm{CH}_{2}\right)_{15} \mathrm{~N}_{(}\left(\mathrm{CH}_{3}\right) 3^{+} \mathrm{Br}^{-}$

3. Non-ionic

The molecule contains a polar hydrophilic chain group and a non-polar hydrophobic chain group.

Example: Span (sorbitan esters)

Tweens (polyoxyethylene sorbitan esters) 
The surfactants are often named in relation to their technological application; such as suspending agents, dispersant, emulsifier, detergent and wetting agent.

In the case of monomer dispersion in aqueous solution (suspension polymerization), the surfactant helps the stabilization of the droplet by adsorbing at the monomer-water interface as illustrated in figure 2.2.

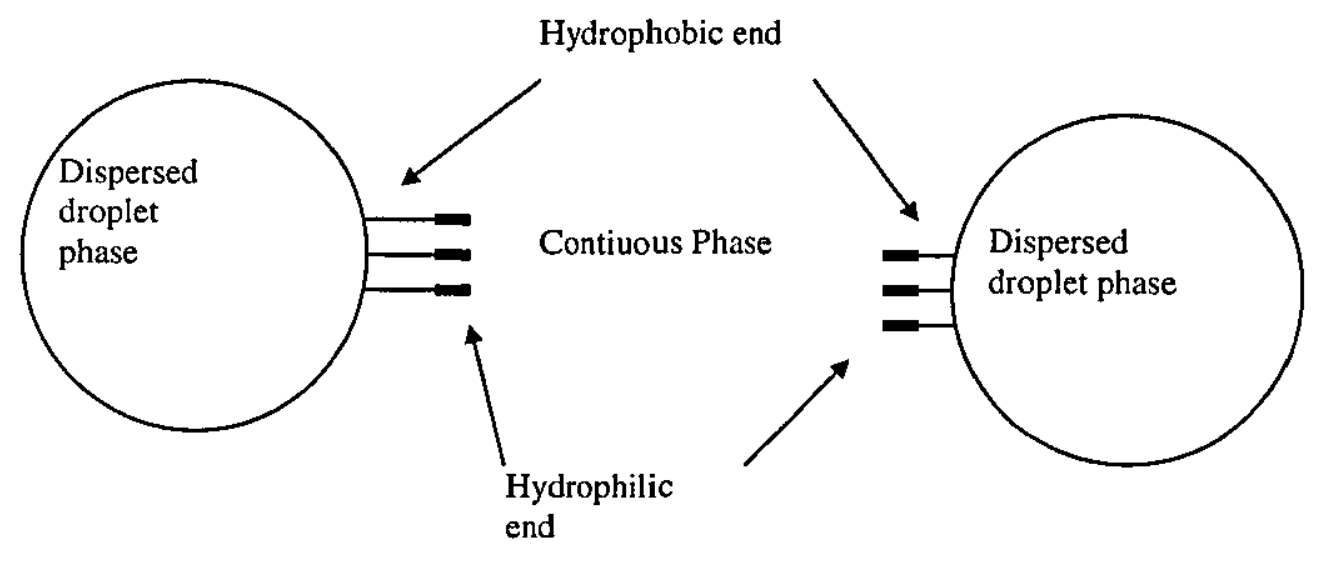

Figure 2.2: $\quad$ Orientation of surfactant molecules at the interface of droplet and continuous phase.

The interface is in the form of a tiny skin, which protect the drops against coalescence. Therefore, one of the main characteristics of a surfactant is its ability to adsorb at the interface in an oriented manner. The efficiency of the adsorbed surfactant is determined by its interfacial concentration, orientation and the energy changes in the system. When the surfactant molecules migrate to the interface, they orientate and adsorb to the interface according to their molecular structure. The decrease of the interfacial tension with the increase of surfactant concentration can be explained by the Gibbs adsorption equation (Adamson, A.W.1967)

$$
q=-\frac{C}{R T} \frac{d \sigma}{d C}
$$


where

$q=$ amount of surfactant per unit area of interface in excess of that present in the bulk solution

$C=$ concentration of the surfactant in the solution

$R=$ the gas constant

$T=$ absolute temperature $\left({ }^{\circ} \mathrm{K}\right)$

Since the surfactant molecules are oriented at the interface of two immiscible liquids to form a surfactant monolayer, the interface contains a higher concentration of surfactant than the solution does. Therefore $q$ is positive and according the equation, $d \sigma / d C$ is negative and the interfacial tension decreases with the increase of the concentration of surfactant.

When a drop collides with another drop, a film of the continuous phase is trapped between the two interfaces. Before coalescence occurs, the film has to be drained out. Hartland, S. (1968) reported that the drainage time was delayed with the increase of the concentration of surfactant. Therefore, the presence of surfactant decreases the average drop size due to the decrease of the rate of coalescence. This is the reason why the type and amount of suspending agents are the important characteristics that influence the size of the droplets. These parameters also continue to effect the morphology and diameter of the polymer particles in suspension polymerisation product. Stephenson, R.C. and Smallwood, P.V. (1989) showed that the choice of stabilizer influenced the mean particle diameter and also influenced the particle porosity.

Walstra, P. (1993) described that if two drops move towards each other and if they were still insufficiently covered by surfactant, the drops will acquire more surfactant at their surface during their approach. However the amount of the surfactant available for adsorption will be lowest at the thinnest film between the droplet. As a result, surfactant moves in the interface toward the side of lowest surface excess. These phenomena causes streaming of the liquid along the surface thus will drive the droplet away from each other. The mechanism only works in a non-equilibrium situation. After the available surfactant molecules are evenly distributed over the droplet surface, the streaming of the surfactant stop. Walsta, P. also suggested that most of 
the coalescence occurs with newly formed drops. The newly formed drops can be from the break up of parent drops or from new established drop at the start of dispersion.

\subsection{Polymerisation Processes}

Polymerisation is a reaction by which small molecules (monomer) combine with each other to form polymer molecules. Commercially, a variety of polymer materials with different properties are being produced to provide a wide range of polymer products. Therefore the polymerisation processes and production technology are always being improved and changes for process efficiency and the improvement of quality of the product are being made. The polymerisation process may be carried out with monomer alone (bulk), in a solvent (solution), in an emulsion in water (emulsion), or in droplets suspended in water (suspension). Polymerisations are also classified as homogeneous or heterogeneous depending on the solubility of polymer in the reaction media.

The polymerisation processes can also be classified in three levels on which modeling is important (Ray, W.H. (1991). The levels are

i- Microscale - Chemical kinetics which include chain growth, chain branching, end group formation and multifunctional sites.

ii- Mesoscale - Physical phenomena which include interphase equlibria, interphase heat and mass transfer, intraphase heat and mass transfer, fluid mechanics and micromixing, and polymer morphology.

iii- Macroscale - Reactor phenomena which include macromixing residence time distribution, overall material and energy balance, particle population balances, heat and mass transfer from the reactor, and reactor dynamic and control.

This section presents only a brief summary of the main polymerisation processes and more attention is focused on the suspension polymerisation process and free radical polymerisation mechanism. 


\subsubsection{Bulk (mass) Polymerisation}

Bulk or mass polymerisation process is the simplest and the most volume efficient of all polymerisation processes with a minimum of contamination of the products. The main components of the process are monomer, initiator and polymer. The bulk polymerisation process, however, is difficult to control. The characteristics of radical chain polymerisation with a highly exothermic nature, the high activation energy involved, and the tendency toward the gel effect combine to make heat dissipation difficult. Since the viscosity of the reaction system increases rapidly at relatively low conversion, a stirring process is needed. The viscosity and exotherm effects make temperature control difficult. Therefore, to overcome the problems, the bulk polymerisation usually is carried out to low conversions with separation and recycling of unreacted monomer. Another method is to carry out the polymerisation in stages, initially to low conversion in a large reactor and to final conversion in thin layers. Despite the difficulties with heat removal and mixing in bulk polymerisation, the process is still used and expanding because of volume efficiency, reduction of contaminants and reduction of expensive equipment for solvent removal, recovery and purification processes. Examples of polymerisation that use bulk process are polymerisation of ethylene, styrene and methyl methacrylate.

\subsubsection{Solution Polymerisation}

In solution polymerisation, a solvent, which acts as a diluent, is used to overcome many of the disadvantages of the bulk polymerisation. There are two types of the solution process:

1) Monomer and polymer are soluble in the diluent. Example: Isobutene in methyl chloride

2) Monomer is soluble but polymer is insoluble. Example: acrylonitrile in chloroform

The presence of solvent improves the transfer of heat of polymerisation, therefore the thermal control is much easier compared to bulk polymerisation. However, the disadvantage of solution polymerisation is the need for removal of the solvent from 
the polymer, which requires a separation process. In addition, the use of inappropriate solvent may affect the polymerisation rate and the molecular weight. Therefore, this process is often avoided unless there are outstanding advantages compared to other processes. The solution polymerisation process is advantageous when the final solution can be used without separating the solvent from the polymer such as in the manufacture of protective coatings and in the textile industry. Vinyl acetate, acrylonitrile and alkyl acrylate are examples of industrial polymerisation in solution.

\subsubsection{Emulsion Polymerisation}

Emulsion polymerisation involves the polymerisation of monomers that are in the form of emulsion (colloidal dispersion). It differs from suspension polymerisation in the type and smaller size of the particles in which polymerisation occurs and in the kind of initiator used. The final product of an emulsion polymerisation, called latex, is often used directly as an emulsion without prior separation of the polymer from water and other components. The products can be employed with appropriate blending operations and applications include coatings, paints, finishes and floor polishes. The main components of the emulsion polymerisation system are monomer, surfactant, water-soluble initiator and water. Monomer is dispersed as droplets in the aqueous surfactant solution and the generation of free radicals in the aqueous phase results in the production of small polymer-containing particles, which are usually the main locus of polymerisation. The surfactant is used in relatively large quantities in order to form micelles in the water phase. The micelles are aggregates of surfactant molecules that are arranged with their hydrophilic ends forming an outer surface adjacent to the water and their hydrophobic ends gathered together at the inside of the micelle. The initial monomer drops eventually disappear and the final product is an aqueous dispersion of very small polymer particles. Emulsion polymerisation offer advantages of lower viscosity and fewer thermal problems and can produce very small particles of polymer. A disadvantage is that the polymer usually contains residual components such as surfactant and initiator decomposition products. Examples of emulsion polymerisation are the polymerisation of styrene, vinyl acetate and vinyl chloride. 


\subsubsection{Suspension Polymerisation}

Suspension polymerisation refers to a process where one or more monomers are dispersed as small particles in a continuos aqueous phase, and polymerised. To keep the monomer phase suspended as small droplets, agitation is applied and small amounts of surfactant are used. The surfactant is generally referred to as suspension stabiliser or suspending agent, which prevents drop coalescence and aggregation of the droplet during polymerisation. If the agitation is stopped, the phases are often separated and the less dense phase rises to the top of the reactor. The volume ratio of the continuous aqueous phase to the dispersed organic phase varies from 1:1 to 6:1. For rapid polymerisation, higher ratios are required to remove heat in a short time. The presence of the aqueous phase provides a heat sink and maintains low suspension viscosity, thereby ensuring good heat transfer, simple temperature control and ease of mixing.

The polymerisation process utilises an initiator, which is soluble in the monomer phase, but insoluble in the continuous phase. Therefore, the polymerisation occurs in the monomer phase and no mass transfer occurs between the monomer and the aqueous phase. Each droplet can be considered to be a miniature bulk polymerisation process. However the individual drops do not retain their unique identity but instead undergo continuous break-up and coalescence. The kinetics of polymerisation within each droplet is the same as those for the corresponding bulk polymerisation. Styrene, methyl methacrylate, vinyl chloride and vinyl acetate are examples of monomer that can be polymerised by a suspension polymerisation process.

The solid polymer formed in the polymerisation may be obtained in the same spherical form in which the monomer was dispersed in the aqueous phase. The size of the monomer droplets and size, porosity and bulk density of the polymer particles can be affected by the water/monomer ratio, type of initiator, type and concentration of stabilisers, agitation and temperature of the polymerisation.

A lot of important commercial polymer resins are manufactured by the suspension polymerisation. Examples are poly(vinyl chloride) and copolymers, styrene resins 
(General purpose polystyrene (GPPS), Expandable polystyerne (EPS), High impact polystyrene (HIPS), poly(styrene-acrylonitrie) (SAN), poly(acrylonitrile-butadienestyrene (ABS), styrenic ion-exchange resin), poly(methyl methacryalate) and copolymers, and poly(vinyl acetate).

Several detailed literature reviews on suspension polymerisation have already been published in the past (Brooks, B.W. (1990),Yuan, H.G. et al. (1991); Hamielec A.E and Tobita, H, and Tobita (1992); and Vivaldo-Lima, E. et al. (1997)). The major aim in suspension polymerisation is the formation of an as uniform as possible dispersion of monomer/polymer droplet (particle size distribution) in the aqeous phase. The final particle size distribution usually between 10 to $5000 \mu \mathrm{m}$, the exact size depending on the monomer type, the type and concentration of stabiliser and the agitation conditions in the reactor (Yuan, H.G. et al. (1991)). These relatively large particles are simply isolated by filtration or sedimentation. Particle morphology is also an important characteristic for the application of the polymer product, particularly in the cases of expandable polystyrene, ion-exchange resins and poly(vinyl chloride).

\subsubsection{Types of Suspension Polymerisation}

There are three main types of suspension polymerisation.

i- $\quad$ Bead (or Pearl) Suspension Polymerisation

In this type, the monomer is a solvent for the polymer. The monomer droplets pass through a viscous syrupy stage to form clear little spheres. An example of bead suspension polymerisation is the polymerisation of styrene to form polystyrene.

ii- $\quad$ Powder Suspension Polymerisation

In this type, monomer does not dissolve in the polymer. Therefore, a bulk precipitation polymerisation occurs in each drop and opaque, irregular grains or powders often are formed. An example of powder suspension polymerisation is the 
production of poly(vinyl chloride).

iii- Mass Suspension Polymerisation

This process has two stages. In the first stage, a rubber is dissolved in the grafting liquid monomer mixture. When the conversion reaches 25 to $30 \%$ the highly viscous reaction mass is transferred to a suspension reactor filled with water containing stabiliser. The reaction proceeds until the desired conversion is reached. An example of mass suspension polymerisation is the production of high-impact polystyrene (HIPS) and acrylonitrile-butadiene-styrene (ABS) resin.

\subsubsection{The Process of Suspension Polymerisation}

The process of suspension polymerisation can be divided into three stages (Yuan, H.G. et al. 1991).

i- In the first stage, a liquid-liquid dispersion exists where the monomer is dispersed in small droplets. The dispersion is stabilised by the combined action of the rotating stirrer and the suspending agent.

ii- The second stage is the establishment of the dynamic equilibrium of break up and coalescence process. This process seems to determine the final particle size. However as the polymerisation proceeds, the viscosity of the monomer-polymer droplet increases. As a result, the dynamic equilibrium between coalescence and break up becomes more difficult.

iii- At the last stage, the polymer particles are solid and they do not stick anymore. After this point, the viscosity of the polymer particle is too high, therefore no break up and coalescence occurs and their diameter remains constant.

In the suspension polymerisation, the viscosity of the monomer phase is low during the early stages. However as the polymerisation proceeds, the viscosity of the monomer-polymer droplet increases. As a result, the dynamic equilibrium between coalescence and break-up becomes more difficult. 


\subsubsection{Advantages and disadvantages of the suspension polymerisation}

Suspension polymerisation has a number of advantages over other polymerisation process:

i- The suspension viscosity is low therefore the efficient mixing is possible.

ii- Better heat control of the polymerisation reaction.

iii- Separation is much easier compared to solution polymerisation.

iv- Lower level of impurity compared to emulsion polymerisation.

$\mathrm{v}$ - If the polymer product is required as a powder form, then the suspension process is suitable because the energy required to disperse the monomer droplets is less than the energy required to break up the finished polymer (compared with bulk polymerisation)

On the other hand, the suspension polymerisation has the following disadvantages:

i- Usually applies only to free radical process

ii- $\quad$ As the viscosity within the droplets rises, the reaction rate increases suddenly. This leads to a surge in heat generation, which does not usually occur in solution or emulsion polymerisation.

iii- The polymer build-up on the reactor wall, baffles and agitators

iv- Larger reactor volume needed compared to bulk polymerisation

$\mathrm{v}$ - Waste water problem compared to bulk polymerisation.

vi- Difficulty in producing homogeneous copolymerisation composition during batch suspension polymerisation.

vii- No commercial continuous process operability yet

\subsubsection{Suspending agents}

The research for suspending agents for suspension polymerisation has been done intensively for different monomer and different conditions of polymerisation, and has resulted in a large patent literature. Among the frequently used stabiliser is poly(vinyl 
alcohol) (PVA). The behavior of the stabiliser is influenced mainly by molecular weight and degree of hydrolysis. The issue of developing a method for selection of the type of PVA as stabiliser in suspension polymerisation was addressed by Mendizabal, E. et al. (1992).

\subsection{Free Radical Chain Polymerization}

\subsubsection{Mechanism}

Suspension polymerisation usually carries the free radical polymerization mechanism. The kinetics mechanism of the free radical chain polymerisation consist of three steps.

i- Initiation

The initiation steps involved two reactions, the first is the production of free radicals from an initiator.

$$
I \stackrel{k_{d}}{\longrightarrow} 2 I
$$

where:

$$
k_{d}=\text { rate constant of initiator decomposition }
$$

The second part of the initiation step involves the addition of the radical to the first monomer molecule to produce the chain initiating species $M_{1}$.

$$
R \cdot+M \stackrel{k_{i}}{\longrightarrow} M_{1}
$$

where:

$M$ represent a monomer molecule

$k_{I}=$ rate constant for the initiation step

In most polymerisations, the second step is much faster than the first step, therefore the production of the free radicals reaction is the rate limiting step in the initiation step and the rate of initiation is then given by 


$$
R_{i}=2 f k_{d}[I]
$$

where:

$$
\begin{aligned}
& {[I]=\text { concentration of the initiator. }} \\
& f=\text { initiator efficiency factor. }
\end{aligned}
$$

ii- Propagation

This step involves the growth of $\mathrm{M}_{1}$ by the successive additions of monomer molecules. Each addition creates a new radical, which is larger by one monomer unit. The successive additions may be represented by

$$
\begin{aligned}
& M_{1} \cdot+M \stackrel{k_{p}}{\longrightarrow} M_{2} . \\
& M_{2} \cdot+M \stackrel{k_{p}}{\longrightarrow} M_{3} . \\
& M_{3} \cdot+M \stackrel{k_{p}}{\longrightarrow} M_{4} .
\end{aligned}
$$

or can be written in general terms as:

$$
M_{n} \cdot+M \stackrel{k_{p}}{\longrightarrow} M_{n+1}
$$

The rate of propagation is given by:

$$
R_{p}=k_{p}\left[M_{n}\right][M]
$$

where:

$k_{p}=$ constant for propagation

$\left[M_{n}\right]=$ concentration of the growing radicals of chain length $n$.

\section{iii- $\quad$ Chain Transfer}

This step is a premature termination of growing polymer by the transfer of a hydrogen or other atom or species to it from some compound present in the system- the 
monomer, initiator or solvent. The reaction may be depicted as

$$
M_{n}+X A \stackrel{k_{r}}{\longrightarrow} M_{n} X+A .
$$

Where XA may be monomer, initiator, solvent or other substance and $\mathrm{X}$ is the species transferred.

The rate of a chain transfer reaction is given by

$$
R_{t r}=k_{t r}\left[M_{n}\right][X A]
$$

where:

$k_{t r}=$ chain transfer rate constant.

$[X A]=$ concentration of the chain transfer agent.

iv- Termination

The termination step can occur as a result of disproportionation or radical combination. The two different modes can be represented in general terms by

$$
\begin{gathered}
M_{n} \cdot+M_{m} \cdot \stackrel{k_{u d}}{\longrightarrow} M_{n}+M_{m} \\
M_{n} \cdot+M_{m} \cdot \stackrel{k_{k !}}{\longrightarrow} M_{n+m}
\end{gathered}
$$

Where $k_{t d}$ and $k_{t c}$ are the rate constant for termination by disproportionation and combination, respectively. The termination step can also expressed by

$$
M_{n} \cdot+M_{m} \stackrel{k_{t}}{\longrightarrow} \text { dead polymer }
$$

The term dead polymer means the cessation of growth for the propagating radical. The rate of termination is given by 


$$
R_{t}=k_{t}\left[\mathrm{M}_{\mathrm{n}}\right]\left[\mathrm{M}_{\mathrm{m}}\right]
$$

where $k_{t}$ is the termination rate constant which is equal to the summation $k_{t d}$ and $k_{t c}$

\subsubsection{Overall Polymerisation Rate}

The overall rate of polymerisation which is synonymous with the rate for monomer disappearance, is given by

$$
\frac{-d[M]}{d t}=R_{i}+R_{p}
$$

However, the number of monomer molecules consumed in the initiation step is far less than in the propagation step. Therefore the rate of initiation can be neglected. The overall rate polymerisation can further be written by applying the steady state assumption which states that the concentration of radicals stays constant and therefore the rate of initiation must be the same as the rate of termination. Therefore equation for rate of initiation can be written as:

$$
R_{i}=2 f k_{d}[I]=2 k_{t}[M \cdot]^{2}
$$

and

$$
[M \cdot]=\left(\frac{R_{i}}{2 k_{t}}\right)^{1 / 2}=\left(\frac{2 f k_{d}[I]}{2 k_{t}}\right)^{1 / 2}
$$

Therefore by substituting this equation into the overall polymerisation rate, the equation becomes

$$
\frac{-d[M]}{d t}=R_{p}=k_{p}[M]\left(\frac{2 f k_{d}[I]}{2 k_{t}}\right)^{1 / 2}
$$




\subsubsection{Degree of Polymerisation}

The number-average degree of polymerisation, $D P_{n}$, is defined as the average number of monomer molecules contained in a polymer molecule. While the number-average molecular weight of a polymer is given by

$$
M_{n}=M_{o} D P_{n}
$$

where

$$
M_{o}=\text { molecular weight of the monomer. }
$$

The degree of polymerisation can be obtained by dividing the polymerisation rate by the sum of the rates of all chain-breaking reactions. The chain-breaking reactions include the termination mode plus all chain transfer reactions. For the case of chain transfer by monomer only, the equation for average-degree of polymerisation can be written as,

$$
\frac{1}{D P_{n}}=\frac{\frac{1}{2} R_{t c}+R_{t d}}{R_{p}}+\frac{k_{t r}{ }_{M}}{k_{p}}
$$

where $k_{t r, M}$ is the chain transfer rate constant for monomer

\subsubsection{The gel effects}

At the early stage of polymerisation, there is a fairly constant reaction speed. As the polymerisation proceed, the viscosity increases, and the rate of polymerisation accelerate. This phenomena is called gel effect or Trommsdorff effect, where due to the effect of viscosity, the chain termination rate by reaction of growing chains becomes diffusion controlled. The gel effect has been attributed to decrease in termination rate constant $\left(k_{t}\right)$. Therefore the chain termination rate is slower than the rates of chain growth. This leads to higher molecular weights and wider molecular weight distribution than formed at low conversion. 


\subsubsection{Copolymerisation}

Copolymerisation is a process in which two monomers are simultaneously polymerised. The product is a copolymer, which contains units of both monomers incorporated into each molecule. The two monomers enter into the copolymer in overall amounts determined by their relative concentrations and reactivities. By considering the case of copolymerisation of the two monomers $M_{1}$ and $M_{2}$, four propagation reactions are then possible.

$$
\begin{aligned}
& M_{1}+M_{1} \stackrel{k_{11}}{\longrightarrow} M_{1} . \\
& M_{1} \cdot+M_{2} \stackrel{k_{12}}{\longrightarrow} M_{2} . \\
& M_{2}+M_{1} \stackrel{k_{21}}{\longrightarrow} M_{1} . \\
& M_{2}+M_{2} \stackrel{k_{22}}{\longrightarrow} M_{2} .
\end{aligned}
$$

where

$\mathrm{k}_{11}=$ rate constant for a propagating chain ending in $\mathrm{M}_{1}$ adding to monomer $\mathrm{M}_{\mathrm{l}}$,

$\mathrm{k}_{12}=$ rate constant for a propagating chain ending in $\mathrm{M}_{1}$ adding to monomer $\mathrm{M}_{2}$, and so on.

The rates of disappearance of the two monomers are given by

$$
\begin{aligned}
& -\frac{d\left[M_{1}\right]}{d t}=k_{11}\left[M_{1} \cdot\right]\left[M_{1}\right]+k_{21}\left[M_{2} \cdot\right]\left[M_{1}\right] \\
& -\frac{d\left[M_{2}\right]}{d t}=k_{12}\left[M_{1} \cdot\right]\left[M_{2}\right]+k_{22}\left[M_{2} \cdot\right]\left[M_{2}\right]
\end{aligned}
$$

Dividing equation [2.87] by equation [2.88] and by assuming steady state concentration, yields the ratio of the rates at which the two monomers enter the copolymer, that is, the copolymer composition, as

$$
\frac{d\left[M_{1}\right]}{d\left[M_{2}\right]}=\frac{\left[M_{1}\right]\left(r_{1}\left[M_{1}\right]+\left[M_{2}\right]\right)}{\left.\left[M_{2}\right] 9\left[M_{1}\right]+r_{2}\left[M_{2}\right]\right)}
$$


where the parameters $r_{1}$ and $r_{2}$ are defined by

$$
r_{1}=\frac{k_{11}}{k_{12}} \quad \text { and } \quad r_{2}=\frac{k_{22}}{k_{21}}
$$

Equation [2.56] is known as the copolymer composition equation. The copolymer composition is the molar ratio of the two monomers units in the copolymer, $d\left[M_{1}\right] / d\left[M_{2}\right]$, being related to the concentration of the two monomers in the feed, $\left[M_{l}\right]$ and $\left[M_{2}\right]$. This equation gives the instantaneous copolymer composition, which is the composition of the copolymer formed from a particular feed composition. The comonomer feed changes in composition as one of the monomers preferentially enters the copolymer. Therefore, there is a drift in the comonomer composition toward the less reactive monomer as the degree of conversion increases. This results in a similar variation of copolymer composition with conversion. In order to minimize the heterogeneity in the copolymer composition, one approach is to maintain the feed concentration approximately constant. This can be done by batchwise or continuous addition of the more reactive monomer to the process. 


\section{CHAPTER 3}

\section{EXPERIMENTAL WORK}

\subsection{Apparatus}

\subsubsection{Liquid-liquid dispersion apparatus}

The experiments were designed for the study of the liquid-liquid dispersion using simulated dispersed-phase as discussed in section 1.2 part A. The two-phase liquid-liquid dispersions were agitated by using rotating stirrers. The agitation action inside the liquid mixtures has a major effect on coalescence and redispersion of drops. Other effects include the increase of interfacial surface area by dispersing one liquid in another.

The apparatus used in the liquid-liquid dispersion experiments consisted of glass reaction vessel of $700 \mathrm{ml}$ with a curved bottom and was fitted with flat flange lid with four sockets. A stainless steel propeller type impeller with four blades was used for mixing the liquid dispersion. This type of impeller moves the liquid axially up and down so that the flow becomes turbulent throughout the vessel. It is also suited for making low or medium viscosity dispersions. To assist a more efficient dispersion and to maintain fairly uniform drops, four equally spaced stainless steel baffles were located in the vessel. The impeller speed was controlled by a variable speed motor 
and the speed was monitored by a tachometer. The glass vessel was positioned in a constant temperature water bath (square tank). The dispersion temperatures were measured directly by using thermocouple located in the vessel and connected to a temperature indicator. For sampling purposes, a syringe $(5 \mathrm{ml})$ with a long needle $(1$ mm diameter) was used to draw samples from the vessel. The detailed description of the reaction vessel with the other apparatus attached to it is shown in Figure 3.1. Specifications and models of some of the equipment used were as follows:

$\begin{array}{ll}\text { i- } & \text { Stainless steel water bath } \\ \text { ii- } & \text { Temperature heater/controller - Grant V } \\ \text { iii- } & \text { Baffles - four stainless steel baffles (Figure 3.2a) } \\ \text { iv- } & \text { Stirrer - Stainless steel propeller type (Figure 3.2b) } \\ \text { v- } & \text { Stirrer motor - Heidolph RZR 50 } \\ \text { vi- } & \text { Temperature indicator - Digitron Model 27 51-K } \\ \text { vii- } & \text { Thermocouple } \\ \text { viii- } & \text { Tachometer - Model TM-2001 } \\ \text { ix- } & \text { Glass reaction vessel - Quickfit FR700F } \\ \text { x- } & \text { Flat flange lid - Quickfit MAF2/32 } \\ \text { xi- } & \text { Syringe - 5 ml }\end{array}$

\subsection{1a Apparatus set-up for batch mixing experiment}

The set up of the dispersion experiment for batch mixing is fairly simple as shown in figure 3.3. The apparatus consist of one vessel with other parts that discussed in section 3.1.1. Figure 3.4 shows the schematic diagram for fittings through the vessel lid.

\subsection{1b Apparatus set-up for mixing of two dispersion}

The apparatus set-up for this type of experiment was somewhat more complicated than the batch mixing experiments. The apparatus design allowed for the transfer of one liquid dispersion to another liquid dispersion. The set-up of the apparatus is shown in figure 3.5 and the fittings for the vessel lid is shown in figure 3.6 


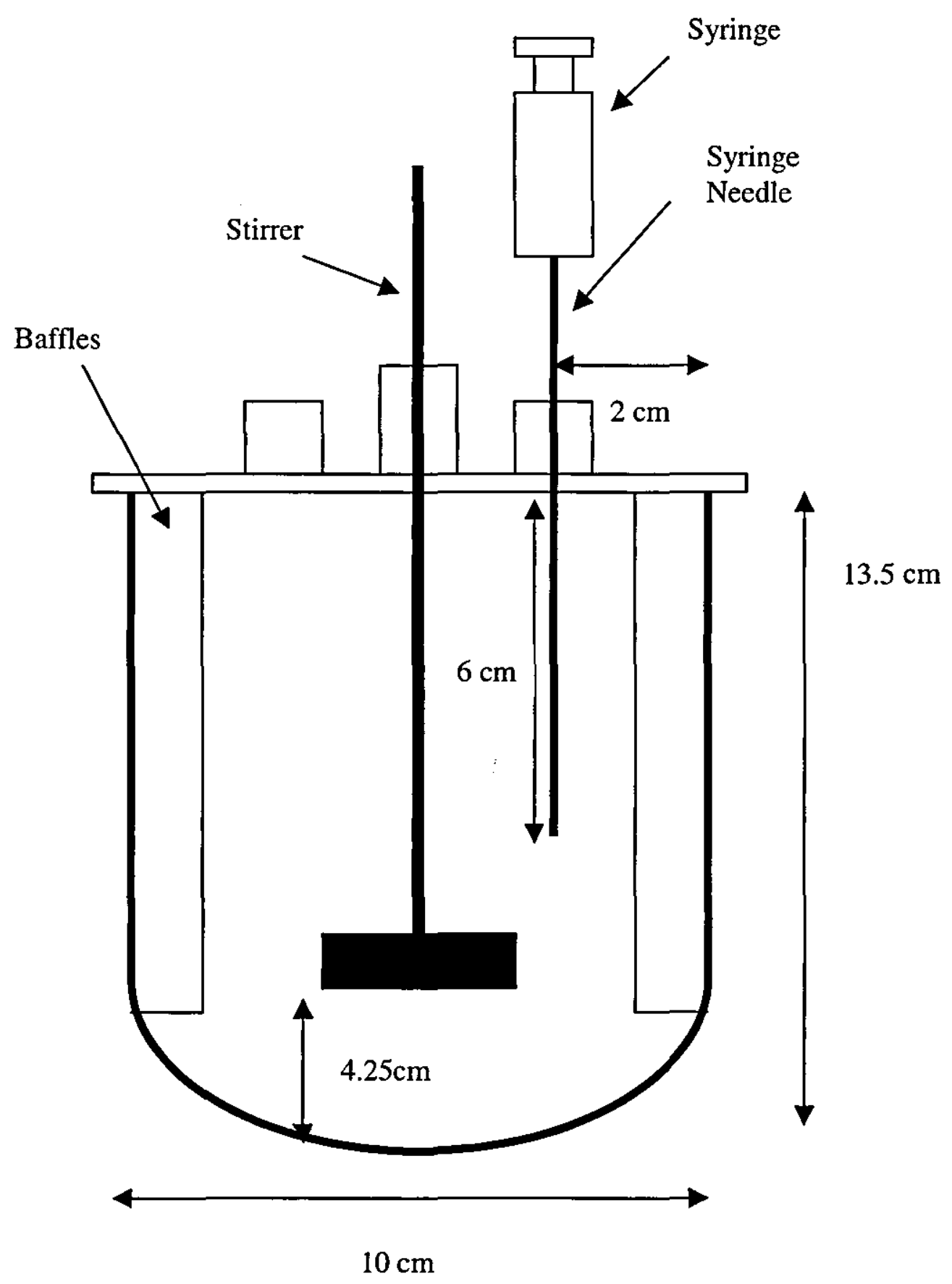

Figure 3.1: Schematic diagram of reaction vessel 


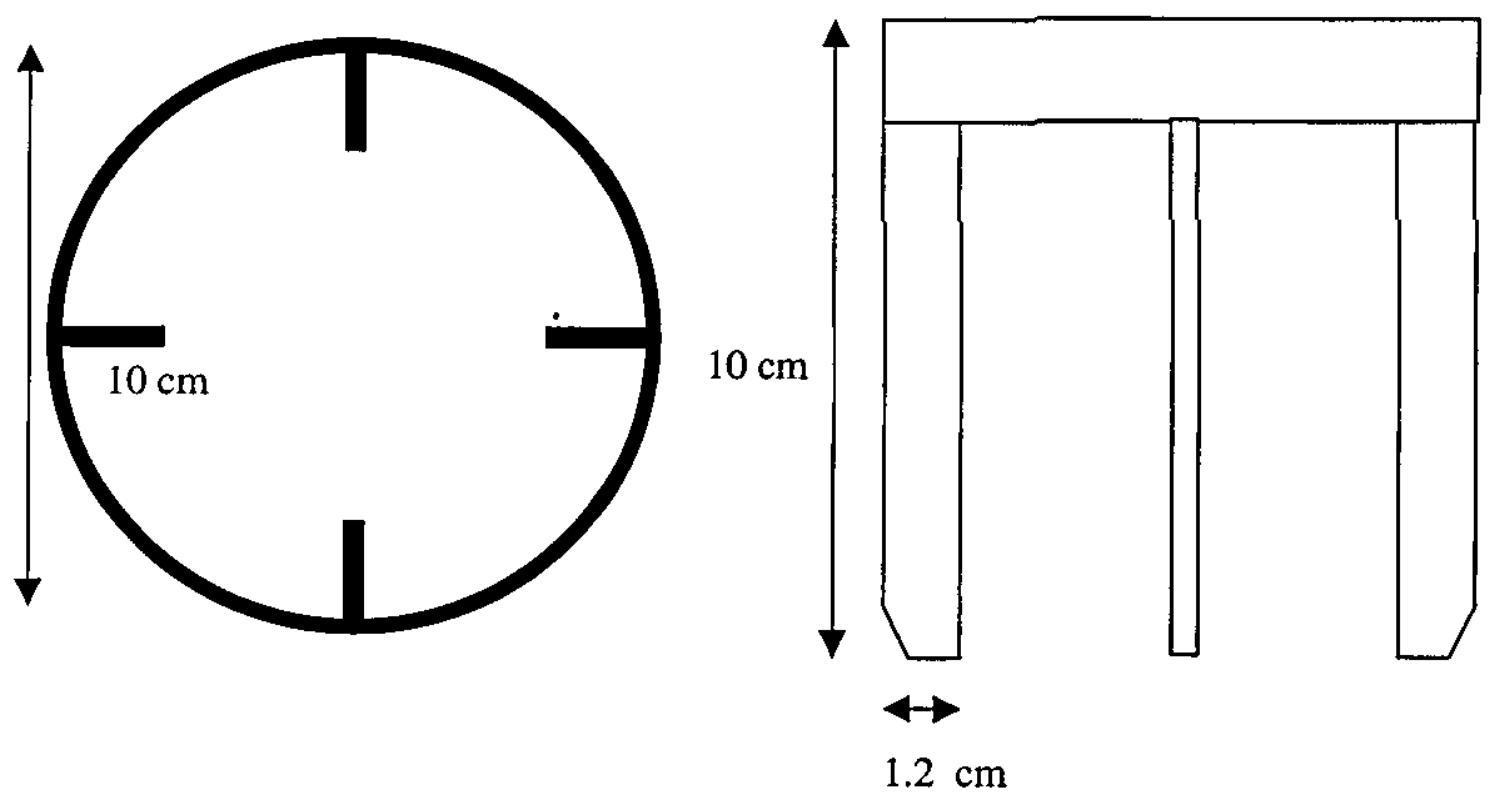

Figure 3.2a: Schematic diagram of baffles

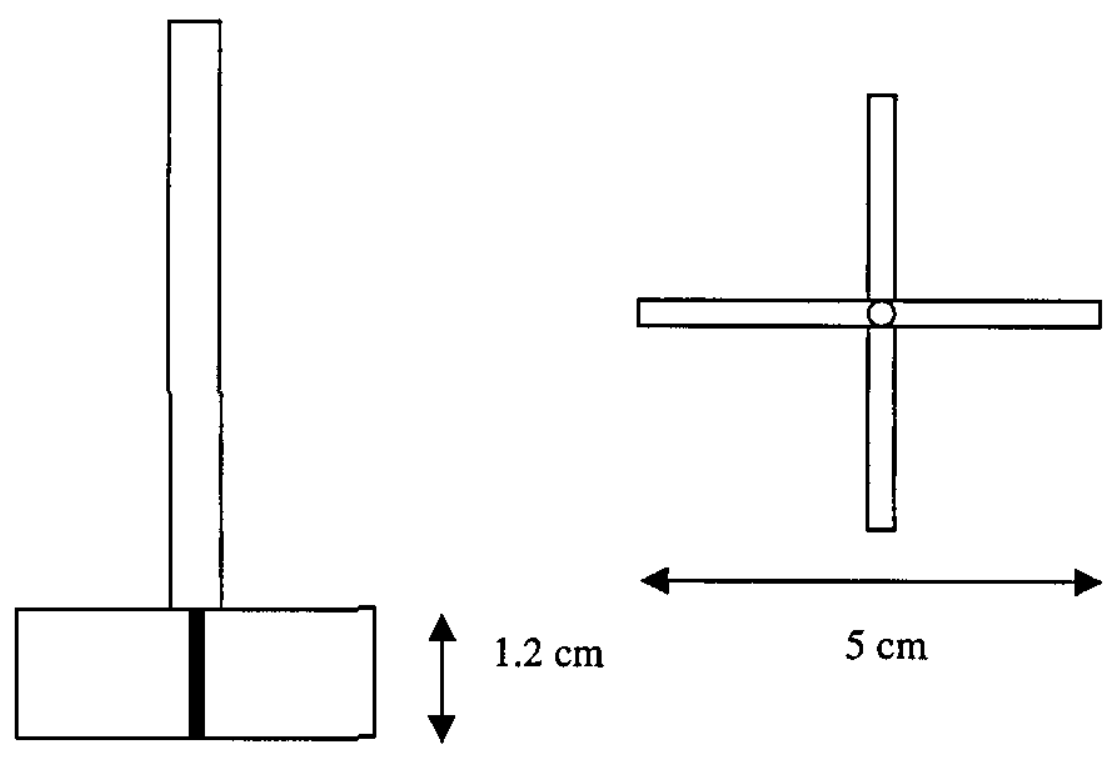

Figure 3.2b: Schematic diagram of stirrer 


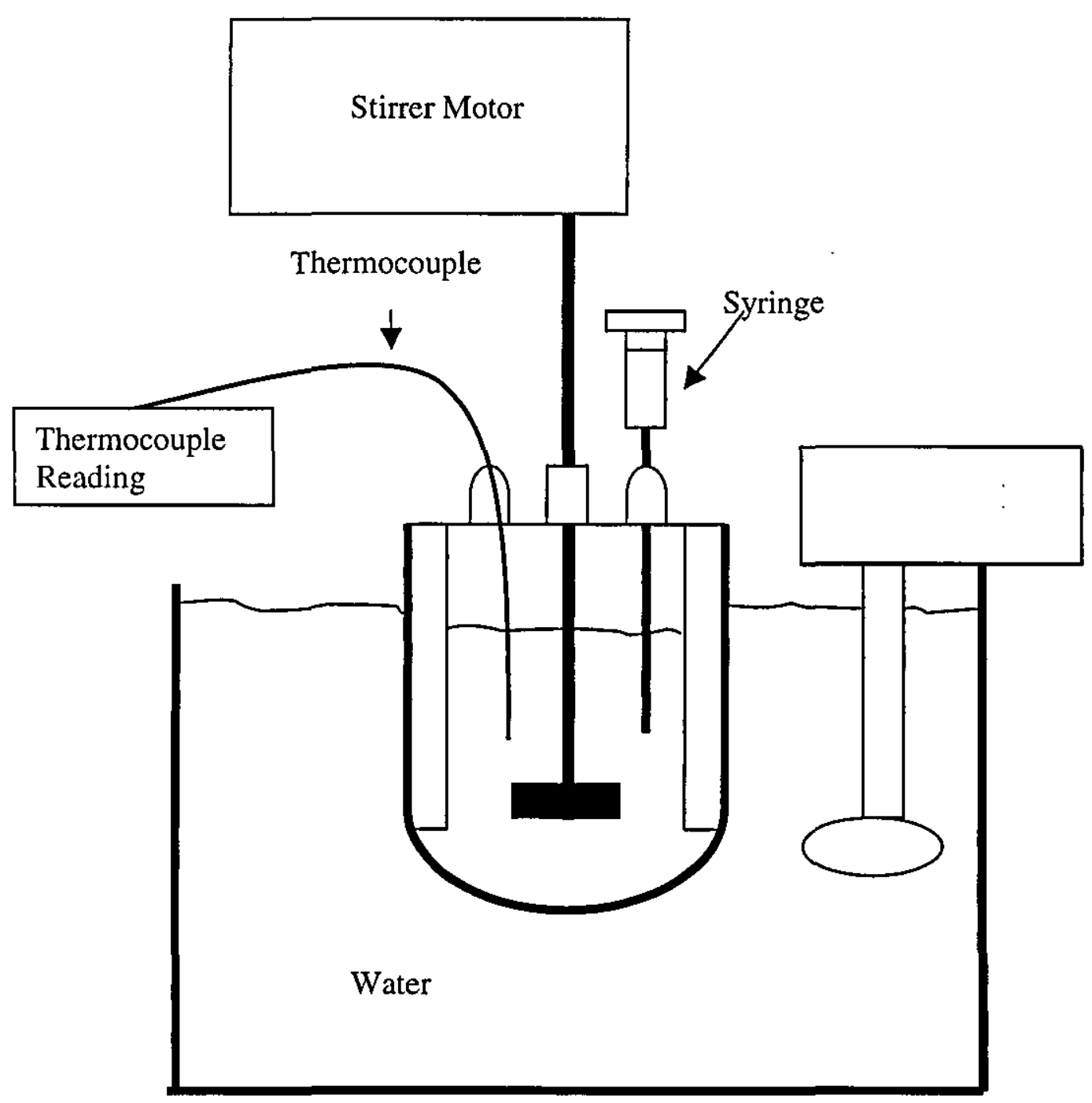

Figure 3.3: Schematic set-up diagram of batch mixing experiments 


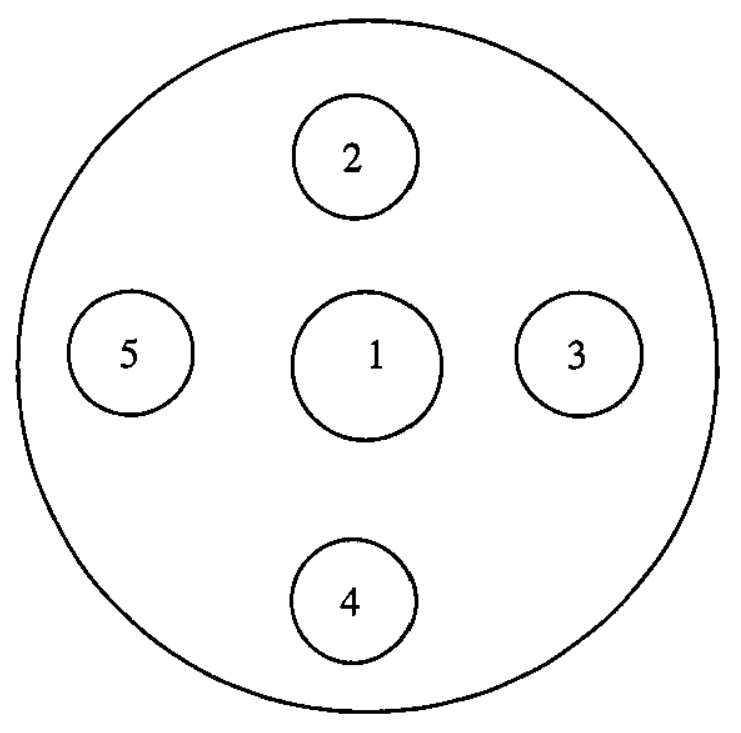

\begin{tabular}{|c|l|}
\hline Socket & \multicolumn{1}{|c|}{ Purpose } \\
\hline 1 & Stirrer \\
\hline 2 & Inlet of liquid \\
\hline 3 & Thermocouple \\
\hline 4 & Inlet of sampling needle \\
\hline 5 & Closed \\
\hline
\end{tabular}

Figure 3.4: Schematic diagram for fittings through the five-neck glass vessel lid (Batch mixing experiments) 


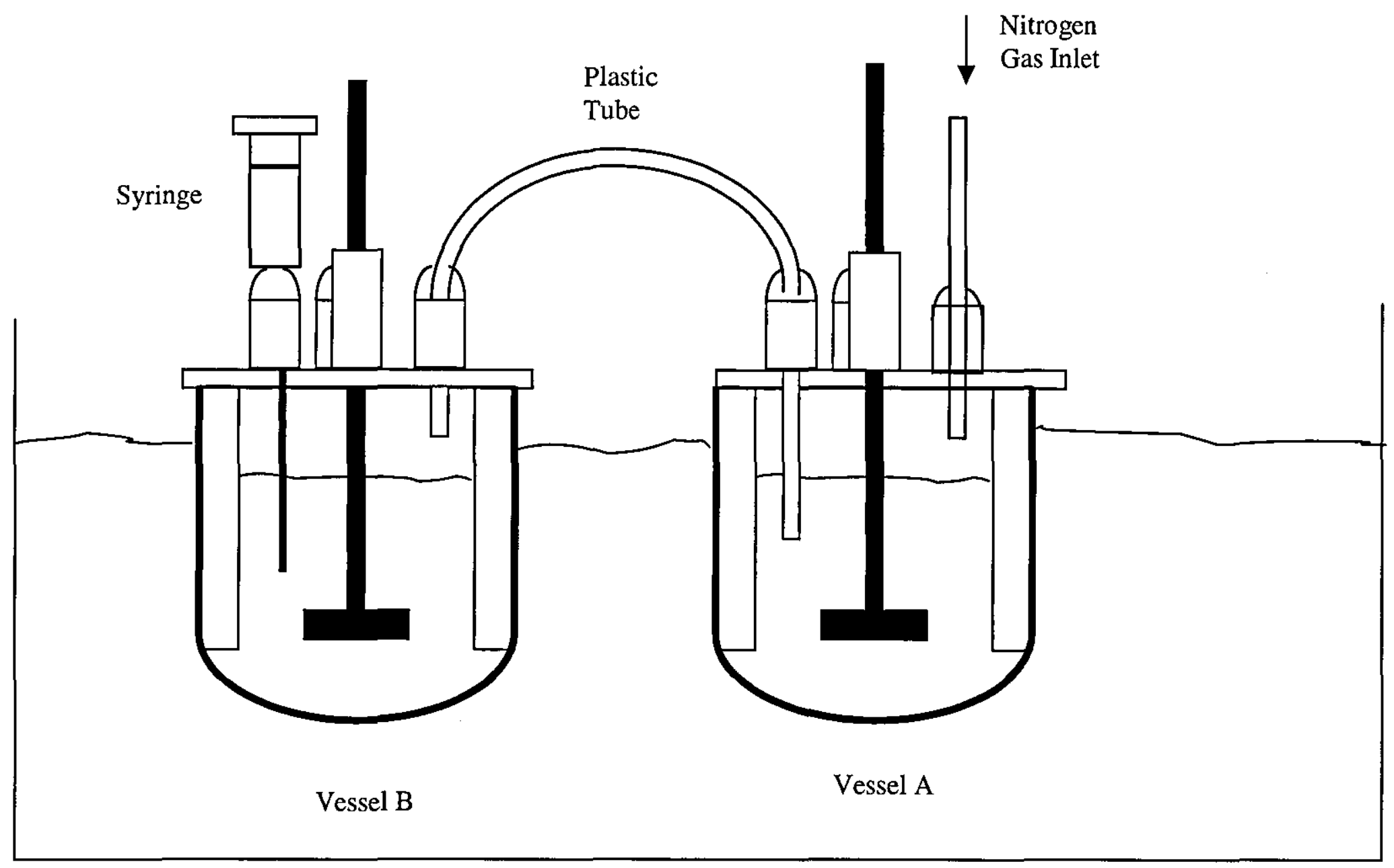

Figure 3.5: Schematic set-up diagram of mixing of two dispersions experiments 


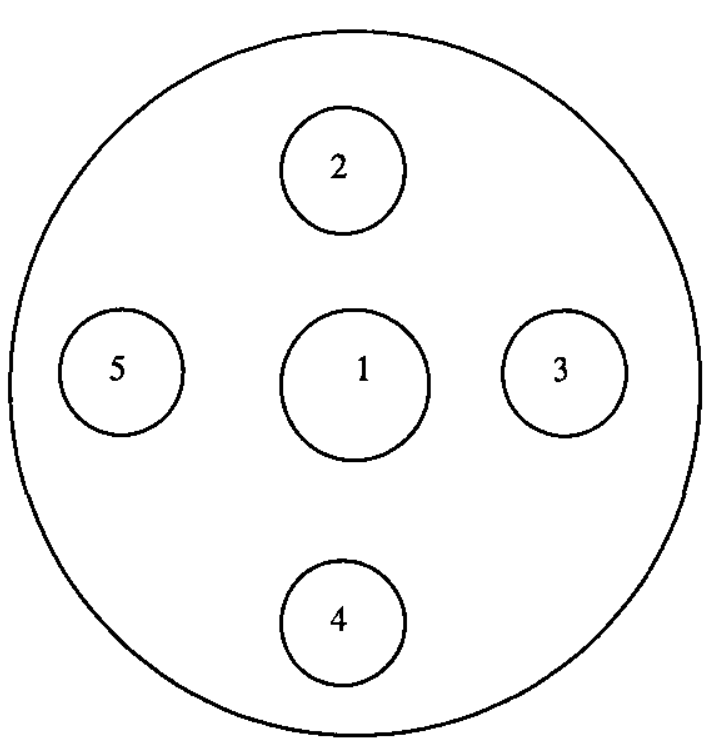

Vessel B

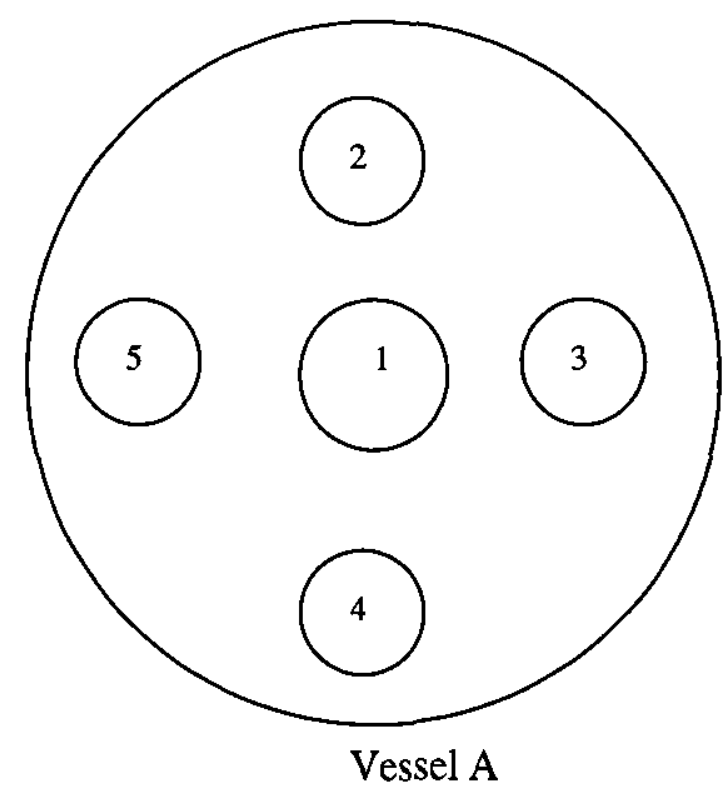

\begin{tabular}{|c|l|l|}
\hline Socket & Purpose (Vessel B) & Purpose (Vessel A) \\
\hline 1 & Inlet of stirrer & Stirrer \\
\hline 2 & Closed & Inlet of Nitrogen Gas \\
\hline 3 & Inlet tube from vessel A & Thermocouple \\
\hline 4 & Syringe needle & Glass valve \\
\hline 5 & Vent & Outlet tube to vessel B \\
\hline
\end{tabular}

Figure 3.6: Schematic diagram for fittings through glass vessel lids in mixing of two dispersion experiments 
The figure shows how the dispersion from one vessel was transferred to another vessel. The apparatus consist of two vessels with each vessel having the same specification as described in section 3.1.1. Nitrogen gas is supplied through a tube to vessel $\mathrm{A}$ to provide higher pressure in the vessel. The liquid from vessel $\mathrm{A}$ is transferred to vessel $B$ through a tube. The tube tip in vessel $A$ is positioned at the bottom of the vessel while for vessel $B$ it is positioned on top of the vessel. The detailed procedure of the system will be discussed in the experimental method section.

\subsubsection{Apparatus for analysis of drops}

The drop sizes and the extent of mixing between drops were initially measured by using an optical microscope (BIOLAM). The dispersions were sufficiently stable for sizes to be measured outside the agitated vessel. A sample was drawn quickly by a syringe into the cavity of a slide glass with $1 \mathrm{~mm}$ depth and was covered by a square piece of glass. The slide glass was then placed under the pre-focused microscope. A PRACTICA camera, which was attached to the microscope, was used to photograph the sample. Then the image (negative film) of the droplet was projected onto the opal screen using a slide projector and the drop diameters were measured manually. This method was used in the preliminary experiments.

Later, the drop sizes were analysed by using a microscope with a video system. A colour video, which was attached to the microscope, was used to obtain the image of the sample. The image produced can be viewed on-line by a colour video monitor and can be recorded by a video cassette recorder. With the assistance of the colour video title generator, each image produced can be labeled for easy identification. The image also can be printed on video graphic. Figure 3.7 shows the schematic diagram of the set-up of the equipments used for the analysis of the dispersion drops and the photograph of this unit is shown in figure 3.8. The specification and models of the equipment used were as follows:
i. Optical Microscope:
LEICA ATC 2000
ii. Colour video camera:
JVC TK-C1381
iii. Colour video monitor:
JVC TM-14E KD 


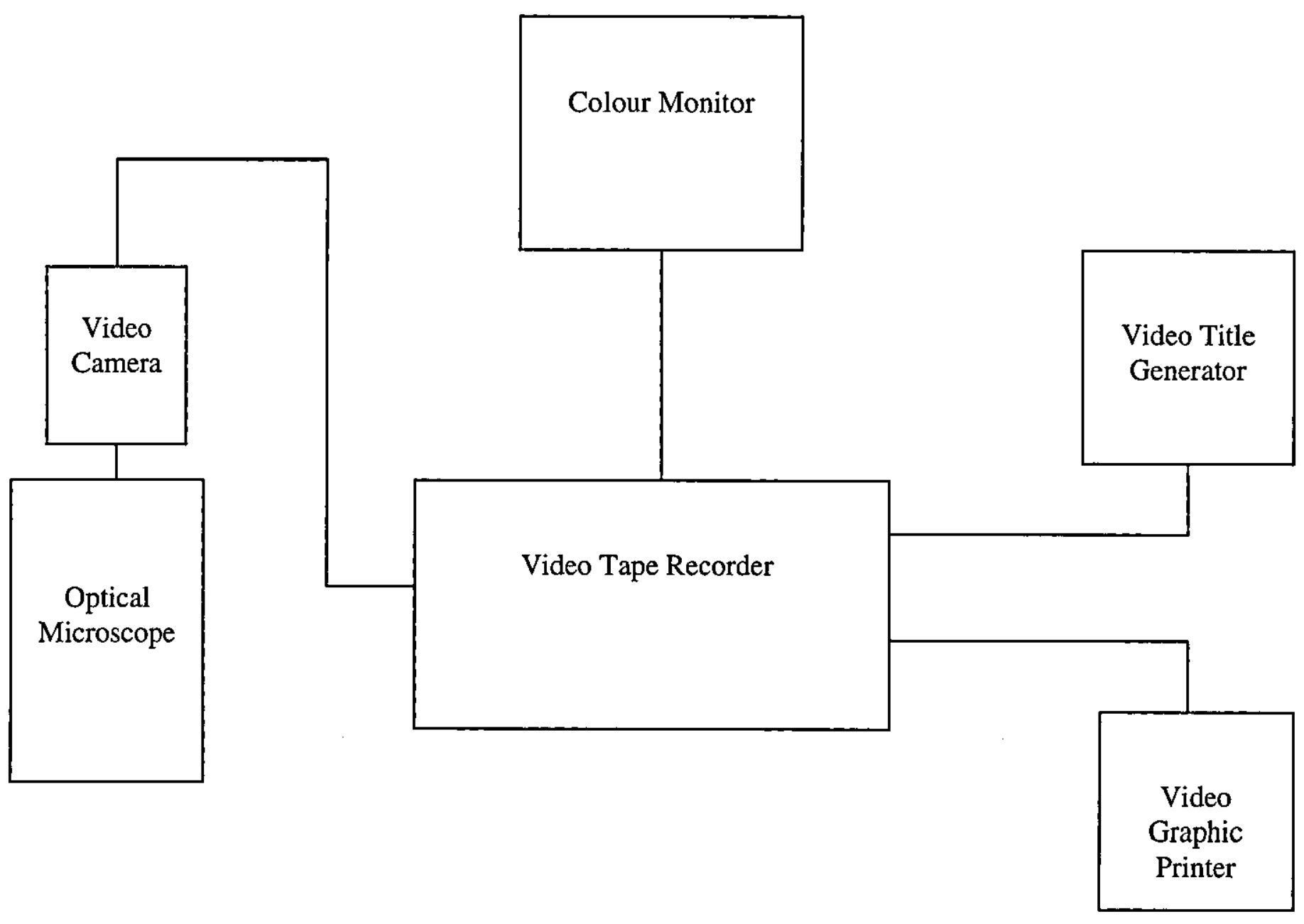

Figure 3.7: Schematic diagram for the set-up of the microscope with the video system 


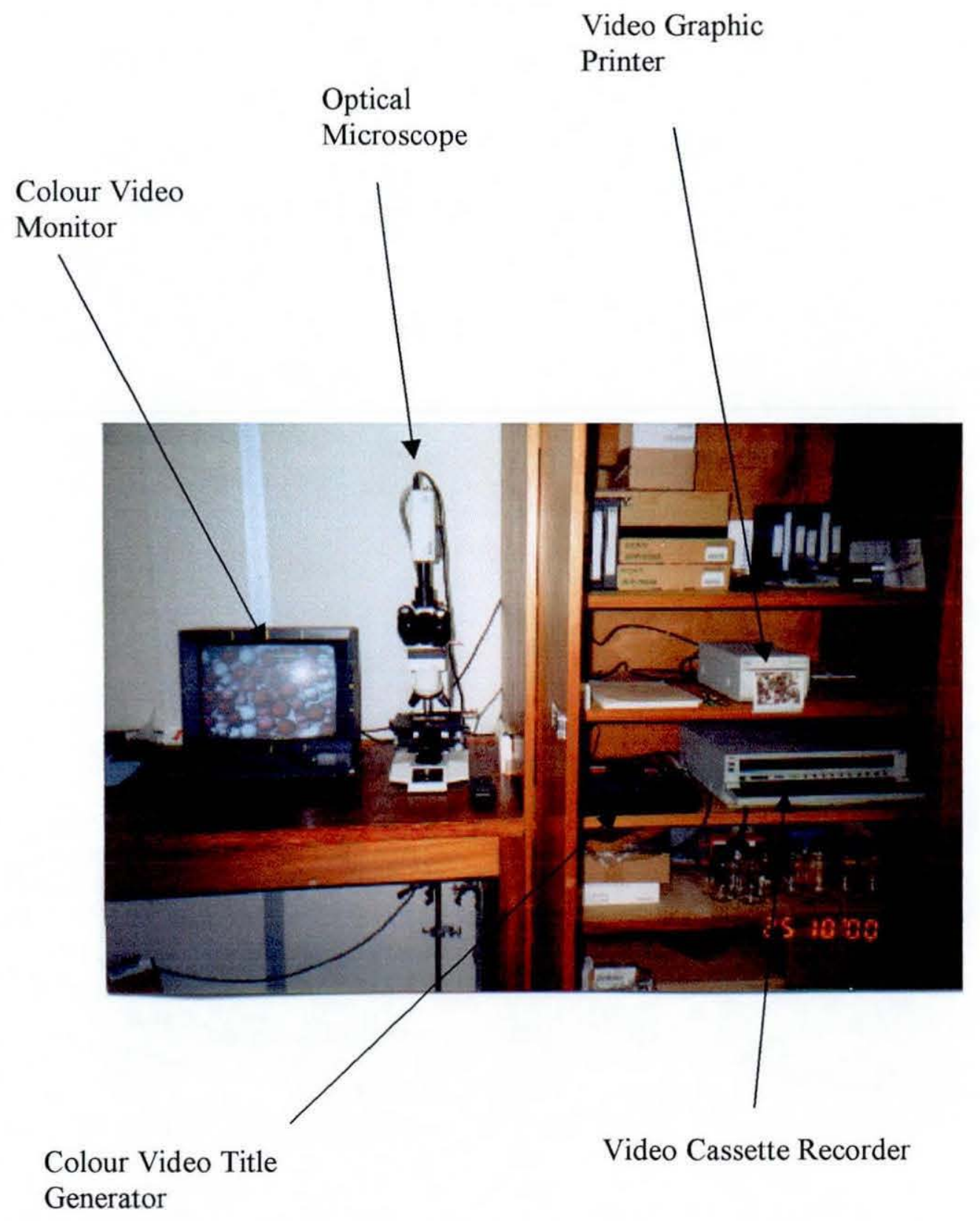

Figure 3.8: Equipments for analysis of drops 

iii. Video cassette recorder SVHS: Panasonic AG 4700
iv. Colour video title generator: VTG 228 Plus
v. Video graphic printer:
Sony UP890 CE

\subsubsection{Characterisation apparatus}

The properties of viscosity, density and surface tension for the liquid dispersion were also determined in this project.

i Viscosity and density measurement

The viscosities of the dispersed phase were measured by using a capillary viscometer and their densities were measured by a volumetric technique.

ii. Surface tension measurement

The surface tension measurements were made using a Du Nouy ring tensiometer at room temperature. The instrument was Digital Balance DB 2KS.

\subsection{Materials}

\subsubsection{Dispersed Phase}

Organic liquids were used as the dispersed phase and, in order to vary the viscosity of the dispersed phase, suitable polymers were dissolved within them. The following liquid solutions were used in the dispersed phase during these experiments:

i. Toluene

The main physical properties of toluene are shown in table 3.1 
Table 3.1: Some physical properties of toluene

\begin{tabular}{|c|c|}
\hline Property & Value \\
\hline Molecular Weight & 92.14 \\
Specific Gravity & 0.867 \\
Boiling Point & $111^{\circ} \mathrm{C}$ \\
Refractive Index (at $20^{\circ} \mathrm{C}$ ) & 1.4960 \\
\hline
\end{tabular}

\section{ii. Styrene}

Styrene monomer was supplied by Fisher Chemicals, it contained stabiliser 10-15 ppm terbutylcatechol. The physical properties of styrene are shown in table 3.2 .

Table 3.2: Some physical properties of styrene

\begin{tabular}{|c|c|}
\hline Property & Value \\
\hline Molecular Weight & 104 \\
Specific Gravity & 0.9060 \\
Boiling Point & $145.2^{\circ} \mathrm{C}$ \\
Refractive Index (at $20^{\circ} \mathrm{C}$ ) & 1.5468 \\
\hline
\end{tabular}

iii. Polystyrene - added polymer to the organic liquid to vary the viscosity.

Supplied by Aldrich

Molecular Weight of 280,000

iv. Sudan red B

It is an organic dye (dye content $=97 \%$ )

Supplied by Aldrich. 


\subsubsection{Continuous Phase}

The continuous phase consisted of distilled water and a surfactant. The surfactants used in these experiments were:

i. Poly(vinyl alcohol) - (PVA)

Trade name: Alcotex Spec "A",

Manufacturer: $\quad$ HARLOW Chemical Co.

Degree of hydrolysis: $72.5 \%$

Molecular Weight: $\quad 75,000$

ii. Polyoxyethylene sorbitan monolaurate - TWEEN 20

Manufacture: SIGMA

Molecular Weight: 1228

iii. Distilled water

\subsection{Experimental Methods}

\subsubsection{Preparation of dispersed phase}

For the preparation of the dispersed phase, the required amount of polystyrene was dissolved in the organic liquid. The liquid solution was then left to stand overnight to dissolve fully. For higher viscosity liquids, more than one day was needed to allow for complete dissolution.

\subsubsection{Preparation of surfactant solution}

For the preparation of the surfactant solution, $4 \mathrm{~g}$ of the surfactant was dissolved in 250 distilled water, using strong agitation. The mixture was strongly agitated for one to two hours depending on the type of surfactant in order to completely dissolve the surfactant. The mixture was left to stand overnight to 
dissolve fully. When the mixture was fully dissolved, it was filtered and stored in a $250 \mathrm{ml}$ flask. This mixture was then diluted as required to form specific amount of aqueous phase. The solution was agitated strongly for about 30 minutes prior to use in the experiment. The concentrated surfactant solution was used within 2 weeks of its preparation. The percentage of the surfactant used was based on the aqueous phase.

\subsubsection{Preparation of dyed solution}

For the preparation of dyed solution, the required amount of the Sudan Red B was added to the liquid before their usage in the mixing experiments. The dyed solution was shaken vigorously to ensure that the dye was fully dissolved. For higher viscosity of polystyrene solution, the dye was added during the preparation of the polymer solution. This was also to ensure that the dyed was fully dissolved.

\subsubsection{Drop size measurement}

i- $\quad$ Photography technique

Several different images were photographed in order to obtain a statistically sufficient number of drops (at least 300 drops). A graticule was also photographed under the same magnification in order to obtain a scale of the image. The scale of the projected images was obtained by projecting the graticule image onto an opal screen. Then the image of the droplets was projected onto the opal screen and the drop diameters were measured manually. The drops were categorised in two types, coloured drops and uncoloured drops. In order to ensure that the same drop was only measured once, the measured drop was marked with a dry marker.

\section{ii- $\quad$ Video technique}

Several different images (at least 300 drops) were saved on the video tape. An image of the graticule was also saved for the scale purposes. The scale was first determined on the colour monitor screen. Then drop diameters and their colour were determined manually on the monitor screen. 


\subsection{Specific Experimental Methods}

\subsubsection{Preliminary study on drop mixing}

These preliminary experiments were to establish some combinations of organic liquids, surfactant and agitation conditions, which give stable drops in aqueous dispersion. The aim at this stage, also, was to gain experience of operating the experimental apparatus for mixing, sampling and photographic procedures. The dispersion consisted of organic liquids as the dispersed phase and a continuous aqueous phase.

After the establishment of the experimental procedure, some quantitative experiments were carried out. The purpose of these experiments was to establish the technique of using a tracer dye to study the extent of drop mixing. These experiments has allowed us:

i. To determine the best technique to be employed to take the images of samples in order to get clear a image of the drops.

ii. To study the extent of the variation of the drop size resulting from changing certain parameters.

SUDAN red ' $B$ ' dye was used as a tracer dye to determine the rate of coalescence between the drops. The preliminary study determined the correct amount of SUDAN red ' $\mathrm{B}$ ' to be used and the appropriate method of dissolving the dye into the solvent. The objective was to differentiate clearly between dyed drops and non-dyed drops. In addition, a clear image must be produced for the analysis of the extent of mixing. A filter was used in the microscope to assist the production of clear images from the photograph. Without the filter, it is very difficult to determine whether the drops, especially the small drops, are coloured or not.

Dispersed phase liquids were prepared and their viscosity's were measured. Table 3.3 shows the values of the viscosity measured by using a capillary viscometer and the densities were measured by a volumetric technique. More experiments were done on the drop mixing for the higher viscosity dispersed phase. The purpose was to gain stable drops in liquid dispersion. 
TABLE 3.3: Viscosity of polystyrene/toluene solution.

\begin{tabular}{|c|c|c|}
\hline Concentration & Density $\left(\mathrm{gcm}^{-1}\right)$ & Viscosity (cp) \\
\hline Toluene only & 0.859 & 0.54 \\
\hline $10 \mathrm{~g}$ PS in 50ml Toluene & 0.896 & 169 \\
\hline
\end{tabular}

\subsubsection{Study of batch mixing of toluene with stabilised dispersion of polystyrene solution}

The aim of this series of experiments was to study the effect of adding a batch of toluene to stabilised dispersions of polystyrene/toluene solution on the extent of mixing as a function of time. Different viscosity of the stabilised dispersion is the major parameter studied in this experiments. Liquids with two different viscosities were used as the stabilised dispersed phase:
i. Dyed toluene only
ii. Dyed solution of $10 \mathrm{~g}$ Polystyrene in $50 \mathrm{ml}$ Toluene

Following are the experimental condition used in this experiment:

$\begin{array}{ll}\text { Premixing dispersed phase volume: } & 25 \mathrm{ml} \\ \text { Added batch of toluene volume: } & 25 \mathrm{ml} \\ \text { Continuous phase volume: } & 450 \mathrm{ml} \\ \text { Temperature: } & 30^{\circ} \mathrm{C} \\ \text { Surfactant: } & 0.02 \mathrm{wt} \% \text { PVA }\end{array}$

The $25 \mathrm{ml}$ of dyed liquid was first dispersed in the $450 \mathrm{ml}$ aqueous phase for 60 minutes before adding another $25 \mathrm{ml}$ of dyed toluene. Then drops of the dispersion were photographed at $5,15,30,45,60,90$ and 120 minutes in order to study the extent of mixing between the drops. The ratio of the volume of the dyed droplets $\left(\mathrm{V}_{\mathrm{c}}\right)$ 
mixing. Drop size distributions were also plotted in order to analyse the drop behaviour. Care must be taken in observing the drop size distribution since the plots were not normalised. The total volume of the drop size of the samples taken at different times were not the same.

\subsubsection{Study of extent of mixing between two dispersion}

The purpose of these series of experiments was to study the effect of mixing of two dispersions on the extent of mixing. The set-up of the experiments was shown in figure 3.4. The experiment started with the premixing of two dispersions with the same proportion of dispersed phase. Following are the experimental conditions that used in these experiments.

Condition before mixing of the two dispersions

Volume of dispersed phase: $25 \mathrm{ml}$

Volume of continuous phase:

Stirrer speed:

Temperature:

Condition after mixing of the dispersion

Volume of dispersed phase: $50 \mathrm{ml}$

Volume of continuous phase:

Stirrer speed:

Temperature:
$225 \mathrm{ml}$ $350 \mathrm{rpm}$ $30^{\circ} \mathrm{C}$

Dispersions were agitated separately for 1 hour before the mixing was carried out. At 1 hour, the whole dispersion from vessel A was transferred to vessel B. This was done supplying nitrogen gas to the vessel. Vessel A was completely sealed except for the tube that connected it to vessel B. For vessel B, nitrogen was allowed to flow out from the vessel through a vent. The nitrogen supply to vessel $A$ forced the liquid to flow to vessel $B$. The flow of the liquid was controlled by a glass valve situated on the top of the vessel. During the transfer of the liquid, the stirrer in vessel A was 
stopped and after the transfer of the liquid had finished, the stirrer speed in vessel B was increased to $500 \mathrm{rpm}$. The lower speed before the mixing was to ensure that the drop size obtained was not too small since the total volume of dispersion before the mixing was smaller than after the mixing.

\subsubsection{Colour contrast observation on the optical microscope}

A series of experiments have been done to determine the limitation of optical microscopy on the image contrast in distinguishing between coloured and uncoloured drops. This study was carried out with the optical microscope that was using the video system (all the detailed studies used the system). Each experiment was conducted by dissolving the dye in dispersed phase and agitating the dispersion for 1 hour. Then the drops were observed under the microscope and on the monitor to see their colour image. Two sets of experiment were done and their compositions are listed in Table 3.4 .

Table 3.4 Experimental condition for colour contrast experiments

\begin{tabular}{|l|l|l|}
\hline & Experiment 3.1A & Experiment 3.1B \\
\hline Dispersed phase & $50 \mathrm{ml}$ Styrene & $50 \mathrm{ml}$ Styrene \\
\hline Continuous phase & $450 \mathrm{ml}$ & $450 \mathrm{ml}$ \\
\hline Surfactant & $0.02 \%$ PVA & $0.02 \%$ Tween 20 \\
\hline Stirrer speed & $500 \mathrm{rpm}$ & $500 \mathrm{rpm}$ \\
\hline Temperature & $30^{\circ} \mathrm{C}$ & $30^{\circ} \mathrm{C}$ \\
\hline
\end{tabular}

An image of big drops of the dispersed phase in both experiments showed that the drop colour was clearly distinguishable under the optical microscope. However the drop colour paled with decrease in drop size. For the drops smaller than $10 \mu \mathrm{m}$ diameter, some of the coloured drops seemed uncoloured on the microscope. Furthermore, the colour of small drops changed with the adjustment of the microscope focus. When the small drops, especially those under $5 \mu \mathrm{m}$ diameter were in focus with the maximum magnification, they looked like coloured dots, however, these coloured dots changed to uncoloured dots with just a little adjustment of the microscope focus. 
This observation shows that the study of drop mixing using an optical microscope is limited if the drop size is too small. Therefore for further experiments, drops sizes in the drop dispersion have to be tailored to be not less than $10 \mu \mathrm{m}$ in order to get reliable results. Since it is very difficult to eliminate the small drops, experimental conditions were chosen so that only a small fraction of the drops was very small.

\subsubsection{Study of batch mixing of styrene with stabilised dispersion of polystyrene solution}

The aim of this series of experiments was to study the effect of adding a batch of styrene to stabilised dispersions of a polystyrene solution. This process would simulate the suspension process of styrene polymerisation. Different viscosities of the stabilised dispersion and different types of surfactant were the major parameters studied in these experiments.

Several dispersed phase liquids were prepared for these experiments and their viscosities were measured. Table 3.5 shows the values of the viscosity measured by using the capillary viscometer and the densities were measured by a volumetric technique.

TABLE 3.5: Viscosity of polystyrene/styrene solution.

\begin{tabular}{|c|c|c|}
\hline Concentration & Density $\left(\mathrm{gcm}^{-1}\right)$ & Viscosity $(\mathrm{cp})$ \\
\hline Styrene & 0.898 & 0.677 \\
\hline 5 wt \% Polystyrene & 0.906 & 7.52 \\
\hline $10 \mathrm{wt} \%$ Polystyrene & 0.913 & 31.0 \\
\hline $15 \mathrm{wt} \%$ Polystyrene & 0.922 & 104 \\
\hline $20 \mathrm{wt} \%$ Polystyrene & 0.931 & 319 \\
\hline
\end{tabular}




\subsubsection{Premixing behaviour of polystyrene solution dispersion}

A series of experiments was carried out to study the behaviour of liquid-liquid dispersion of polystyrene solution before a new batch of styrene was added. Therefore comparison can be made between drop behaviour before and after the addition of the new batch of styrene. Three different viscosities of polystyrene solution were used as dispersed phase in these experiments, they were $5 \mathrm{wt} \%, 10 \mathrm{wt}$ $\%$ and $15 \mathrm{wt} \%$.

The following experimental conditions were used for the experiments:

Dispersed phase volume: $\quad 40 \mathrm{ml}$

Continuous phase volume: $450 \mathrm{ml}$

Temperature: $\quad 30^{\circ} \mathrm{C}$

Agitation speed: $\quad 500 \mathrm{rpm}$

Stabiliser: $\quad 0.02 \mathrm{wt} \%$ PVA

The vessel was filled with the $450 \mathrm{ml}$ of the continuous phase (PVA solution) and was heated to the temperature of $30^{\circ} \mathrm{C}$. At the same time, the stirrer was also operated at the required speed. When the temperature has reached the required value, the dispersed phase was added to the vessel. The timing started at this stage and, at 0,5 , $15,30,60,90,120$ and 150 minutes, samples of the dispersion were taken by the syringe and transferred to the slide glass to be observed using the optical microscope. The images produced were then analysed to determine the drop behaviour.

\subsubsection{Batch mixing of styrene with polystyrene solution}

These experiments were carried out to study the effect of experimental conditions on the coalescence rate between the added batch of styrene and the existing polystyrene solution. The stabilised dispersion was prepared as in the section 3.4.3.1. After 2 hours of premixing of the dispersion, a solution of $10 \mathrm{ml}$ of styrene and $0.1 \mathrm{~g}$ of Sudan Red B was introduced to the dispersion. Then drops of the dispersion images were taken at $5,15,30,45,60,90,120,150$ and 180 minutes in order to study the extent of mixing between the drops. The ratio of the volume of the dyed droplets $\left(\mathrm{V}_{\mathrm{c}}\right)$ 
to the total volume of droplet $\left(\mathrm{V}_{\mathrm{i}}+\mathrm{V}_{\mathrm{c}}\right)$ was determined to measure the extent of mixing.

The following experimental conditions were used in these experiments:

Premixing dispersed phase volume : $40 \mathrm{ml}$

Added batch of styrene volume: $10 \mathrm{ml}$

Continuous phase volume: $\quad 450 \mathrm{ml}$

Temperature: $\quad 30^{\circ} \mathrm{C}$

For the study of agitation speed effects, three different agitation speeds were used, they are $350 \mathrm{rpm}, 500 \mathrm{rpm}$ and $650 \mathrm{rpm}$. The effect of type and concentration of surfactant have also been studied in these experiments. The surfactant used for this study were 0.02 wt \% PVA and 0.02 wt \% Tween 20.

\subsubsection{Study of mixing of two stabilised dispersions}

The aim of this series of experiments was to study the drop mixing behaviour for the mixing between two stabilised dispersions. The set-up of the experiments was shown in figure 3.5. The procedures for these experiments ensured that both experimental conditions were the same for predispersion and after the two dispersions were mixed. Each vessel was filled with $450 \mathrm{ml}$ continuous phase and $50 \mathrm{ml}$ dispersed phase. Vessel A contained the dispersed phase, which was dyed. Both compositions were heated to the temperature of $30^{\circ} \mathrm{C}$ and were dispersed for 120 minutes before mixing of the two dispersions was carried out. At 120 minutes, half of the dispersion in vessel $\mathrm{A}$ was drained out. The drainage was done with the assistance of pressure from the nitrogen supply. Vessel A was completely sealed with one tube immersed in the liquid. The nitrogen supply to the vessel forced the liquid to flow out of the vessel. The flow of the liquid was controlled by a glass valve situated on top of the vessel. The stirrer was still rotating during the draining out of the dispersion to ensure that the same composition of dispersed phase and continuous phase left the vessel. The dispersion was drained out to the measuring cylinder. After $250 \mathrm{ml}$ of drained out, the nitrogen supply was ceased and the stirrer was also stopped. The same procedure was done on vessel $\mathrm{B}$ to drain out $250 \mathrm{ml}$ of the dispersion. 
stopped. The same procedure was done on vessel $\mathrm{B}$ to drain out $250 \mathrm{ml}$ of the dispersion.

Then the tube was connected between vessel $A$ and vessel $B$, with the end of tube in vessel $A$ at the bottom of the vessel to ensure that all the dispersion left in the vessel was transferred to vessel B. Vessel B was completely sealed and vessel A has one hole to allow the transfer of the dispersion. The flow was controlled by a glass valve in vessel A. After the transfer of the dispersion from vessel A to vessel B was done, the stirrer was restarted. The procedure to transfer the dispersion took about 90 seconds. At 5, 15, 30, 45, 60, 90, 120, 150 and 180 minutes after the mixing of the two dispersion was started, images were taken in order to study the extent of mixing between the drops. The ratio of the volume of the dyed droplets $\left(V_{c}\right)$ to the total volume of droplet $\left(V_{i}+V_{c}\right)$ was determined to measure the extent of mixing.

The following experimental conditions were used in these experiments:

$\begin{array}{ll}\text { Premixing dispersed phase volume: } & 50 \mathrm{ml} \\ \text { Premixing continuous volume: } & 450 \mathrm{ml} \\ \text { Temperature: } & 30^{\circ} \mathrm{C} \\ \text { Surfactant: } & 0.02 \mathrm{wt} \% \text { PVA } \\ \text { Stirring speed: } & 350 \mathrm{rpm}\end{array}$

The major variable studied was the viscosity of the dispersed phase. Two types of experiments were done in this study. First was the mixing of two stabilised dispersions of the same viscosities of the dispersed phase. Second was the mixing of two stabilised dispersions with different viscosities of the dispersed phase. Effect of the viscosity of the dispersed phase was the major variable studied.

\subsubsection{Study of the mixing of same viscosities of dispersed phase.}

In these experiments, both dispersions had the same viscosity for the dispersed phase. The viscosity of the dispersed phase used in both dispersions were

i) $\quad 0 \mathrm{wt} \%$ polystyrene solution (styrene only) 
ii) $5 \mathrm{wt} \%$ polystyrene solution

iii) $10 \mathrm{wt} \%$ polystyrene solution

iv) $15 \mathrm{wt} \%$ polystyrene solution

The dispersed phase in vessel A contained Sudan Red B dye. Therefore the extent of mixing could be determined after the mixing of the two dispersions.

Before the mixing of the two dispersions was carried out, images were taken for the two premixed dispersions at 120 minutes i.e. for both coloured and uncoloured dispersions. The purpose was to determine their drop sizes before the mixing occurred. Therefore, comparison can be made for the behaviour before and after the mixing.

\subsubsection{Study of the mixing of different viscosities of dispersed phase.}

The aim of these experiments was to study the effect of different viscosities of the dispersed phase of two dispersions on the rate of mixing. The experimental procedure and conditions were the same as in section 3.4.4.1, except that different viscosities were used for dispersed phases. Following are the different viscosities used for the two dispersions.

i) Mixing of $0 \mathrm{wt} \%$ polystyrene solution (coloured) and $5 \mathrm{wt} \%$ polystyrene solution (uncoloured)

ii) Mixing of $0 \mathrm{wt} \%$ polystyrene solution (coloured) and $10 \mathrm{wt} \%$ polystyrene solution (uncoloured)

iii) Mixing of $0 \mathrm{wt} \%$ polystyrene solution (coloured) and $15 \mathrm{wt} \%$ polystyrene solution (uncoloured).

\subsubsection{Study of comparison between batch mixing and mixing of two dispersions.}

The purpose of these experiments was to compare the drop behaviour in added batch mixing and mixing of two dispersions. The batch mixing experiments were repeated with the same conditions as in dispersed mixing experiments. The 
experiments started with premixing the dispersions for 120 minutes. At 120 minutes, half of the volume of dispersion was drained out and new dispersed and continuous phases were added to the dispersion. These two solutions were not premixed and they were added separately to the dispersion. 


\section{CHAPTER 4}

\section{RESULTS AND DISCUSSION}

\subsection{Preliminary study on drop mixing}

In order to understand the effect of experimental conditions on drop mixing, a variety of experimental approaches have been used in the past. Among these experimental methods, the spread of tracer dye was the most popular. Miller, R.S. et al (1963), Komasawa, I et. al(1971), Verhoff, F.H. et al (1977), Coulaloglou, C.A. and Tavlarides L.L. (1977), Ross, S.L. et al (1978), Zerfa, M. and Brooks, B.W. (1996b). This experimental approach is used in the present study to understand the drop behaviour in this experimental mixing study.

The objective of this sub-section is not to present a detailed discussion of the results but to find the appropriate experimental method for drop mixing using the tracer dye technique. In addition, some observations were made to have an idea on the behaviour of a liquid-liquid dispersion with the addition of new identical dispersed phase material. Toluene was used in these series of preliminary experiments because of lower cost, less toxic and less odour compared to styrene. This study was an experimental approach to understand the relative effect of some experimental conditions. For this purpose, additional toluene containing water insoluble, Sudan Red B dye was added to the non-dyed toluene or polystyrene/toluene solution in the dispersion. After the addition of new toluene to the existing toluene droplets, 
photographs were then taken at different time intervals and the ratio of the volume of the coloured droplets $\left(V_{c}\right)$ to the total volume of toluene in the vessel $\left(V_{c}+V_{i}\right)$ was determined. $V_{i}$ is the total volume of uncoloured droplets. This ratio is called an extent of mixing between coloured and non-coloured drops. This parameter can also represent the efficiency of mixing or coalescence between the drops.

\subsubsection{Drop mixing for added batches of toluene to a stabilised dispersion with a different dispersed phase viscosity}

In these experiments, a batch of toluene was added to a stabilised dispersed phase of a different viscosity. The aim was to study the effects of different viscosity of the dispersed phase on the extent of mixing between the new and the old drops. The initial dispersion with $25 \mathrm{ml}$ of dispersed phase and $450 \mathrm{ml}$ of continuous phase (0.02 wt \% PVA) was started and run for one hour to allow the droplets to stabilise. Then $25 \mathrm{ml}$ of new toluene containing Sudan Red B dye was added to the dispersion. The agitation continued and photographs were taken at $5,15,3045,60,90$ and 120 minutes. Two viscosities of initial dispersed phase were used in these experiments, toluene and a solution of $10 \mathrm{~g}$ Polystyrene/50ml toluene.

The results obtained are plotted in figure 4.1 which shows the variation of extent of mixing as function of stirring time of mixing between coloured and noncoloured drops with different viscosity of dispersed phase. The figure shows that the new batch of toluene mixed with the existing drops in the dispersion. It can be seen that the extent of mixing increases as a function of time for both viscosities of dispersed phase, but at different rates. The coalescence rate for the mixing of the coloured toluene with non-coloured toluene was higher than for the mixing of the polystyrene/toluene solution with toluene. This preliminary experiment could indicate that the viscosity of the dispersed phase affects the coalescence rate. However the analysis of the average drop size and drop size distribution, which could also be important were not yet discussed at this stage. They will be discussed in detail for the drop mixing using styrene in section 4.2 . 


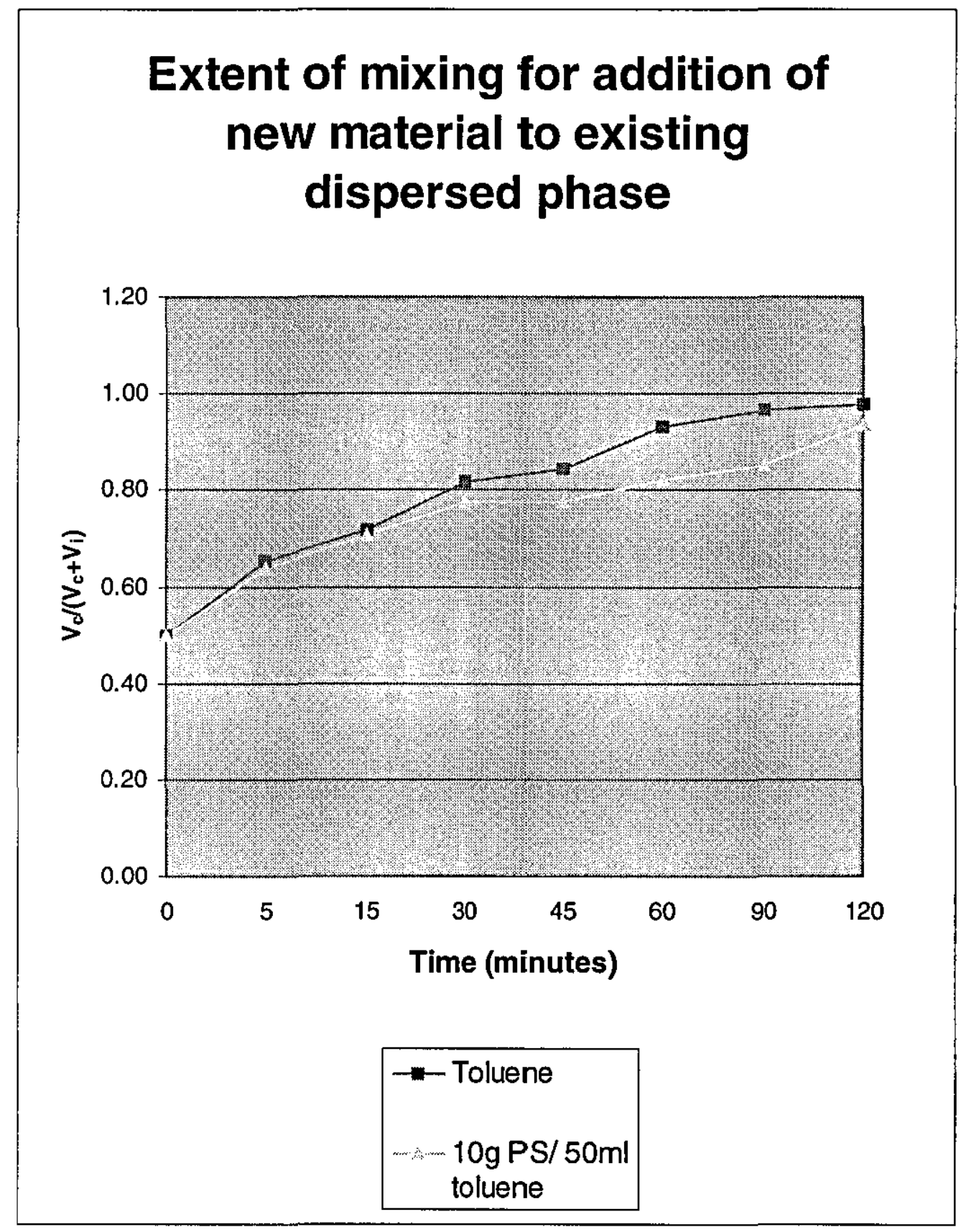

Figure 4.1: Batch mixing of new toluene with the existing dispersed phase using 0.02 wt \% PVA 


\subsubsection{Study of the extent of mixing between two dispersions}

In these experiments, two dispersions of the same dispersed phase fraction were mixed together at a specific time in order to study the mixing rate between them. Before the mixing of the two dispersions, each vessel containing $25 \mathrm{ml}$ drop phase and $225 \mathrm{ml}$ continuous phase was stirred for one hour. The initial stirrer speed was at $350 \mathrm{rpm}$. After one hour, the dispersion without dye was transferred to the other vessel. The stirring speed was then increased to $500 \mathrm{rpm}$ and the extent of mixing was determined as a function of time. The purpose of setting the initial stirrer speed lower was to ensure that the drop size obtained was not too small since the total volume of dispersion before the mixing was smaller than after the mixing. The experiment was repeated, using Tween 20 to study the behaviour of the drop mixing using different surfactant.

\subsubsection{Extent of mixing for two different surfactants}

The aim of these experiments was to compare the effects of surfactant used. In this experiment, a dispersion of toluene without stabiliser (uncoloured) was mixed with the dispersion of toluene (coloured) with surfactant. The stabilised dispersion was transferred to the unstabilised dispersion. Two sets of experiments were done, one for $0.02 \mathrm{wt} \%$ PVA and the other for $0.02 \mathrm{wt} \%$ Tween 20.

The results of the two experiments are shown in figure 4.2. They show that rate of mixing for PVA surfactant was higher than for the Tween 20 surfactant. This shows that Tween 20 surfactant protect the drops more efficiently compared to the PVA surfactant. Figures $4.3 \mathrm{a}$ and $4.3 \mathrm{~b}$ show the drop size distributions for both cases after mixing for 120 minutes, respectively. They show that for the mixing using 0.02 wt \% PVA, the drop size distribution is narrower compared to the mixing using 0.02 wt $\%$ Tween 20. The mean drop size was also lower for the mixing using $0.02 \mathrm{wt} \%$ PVA. These results show that the type of surfactant used affects drop mixing rate between the drops, final drop size and drop size distribution. 


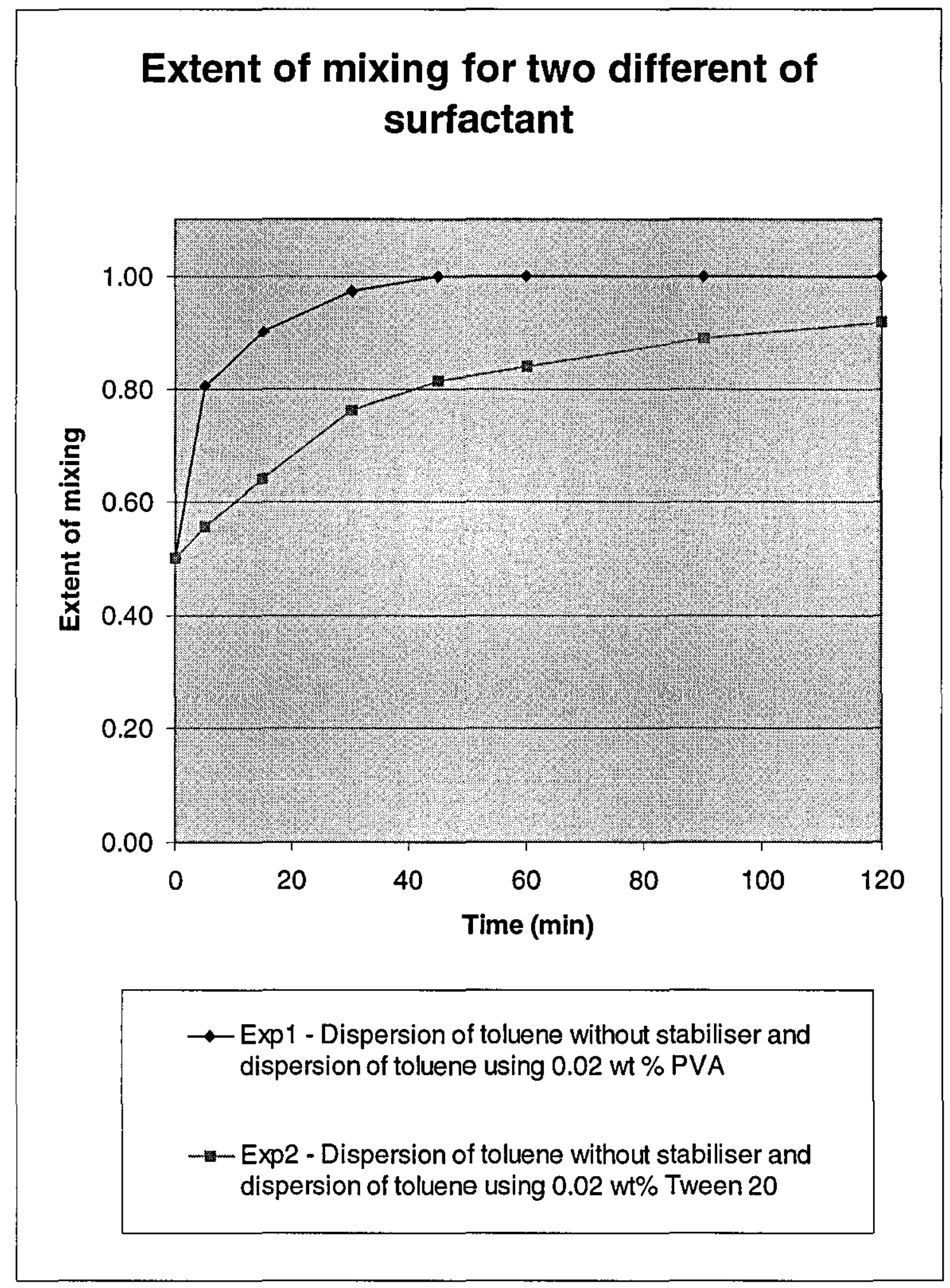

Figure 4.2: Extent of mixing between two dispersion of different surfactant 


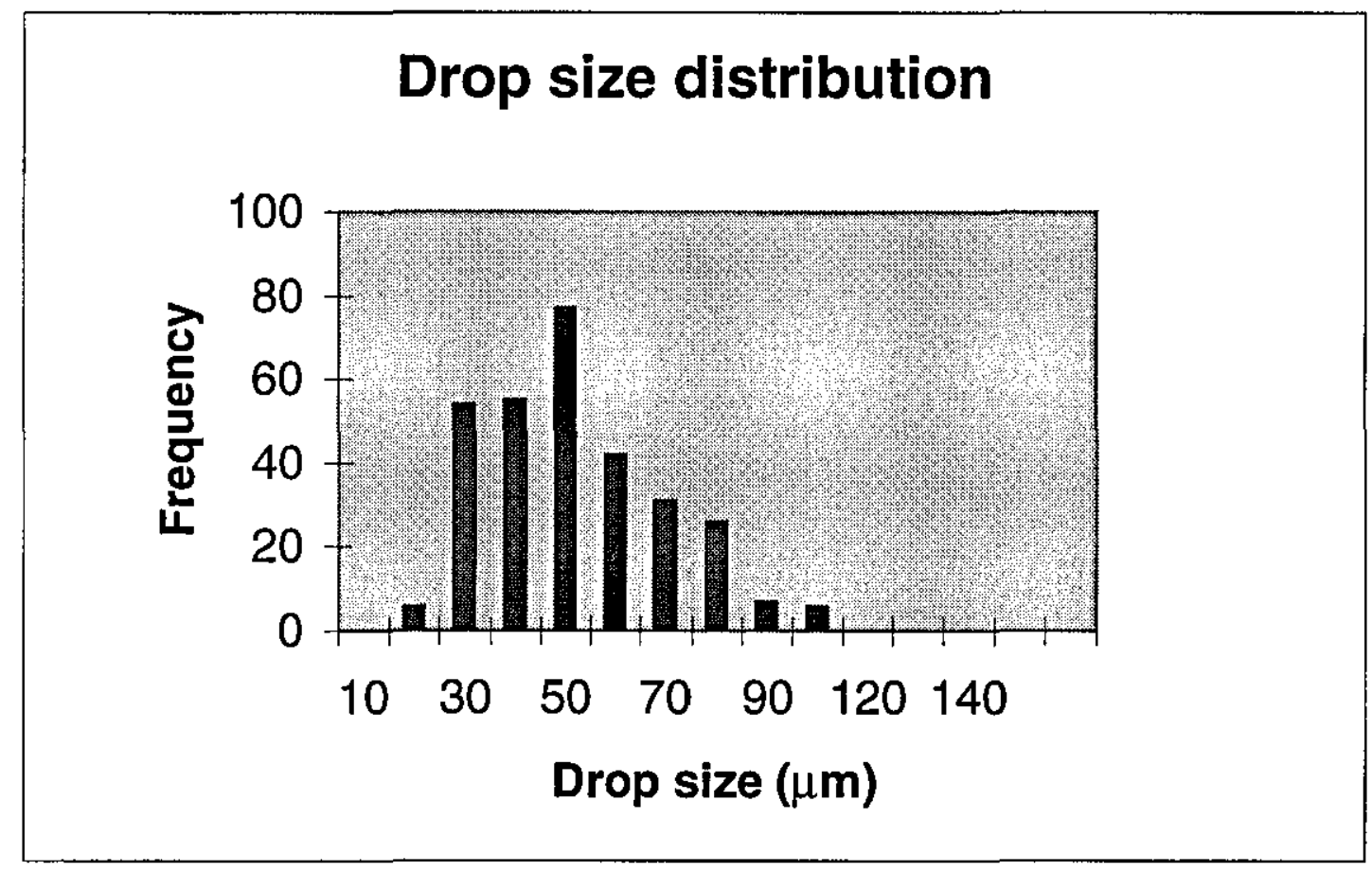

Figure 4.3a: Drop size distribution for mixing of dispersion without stabiliser and dispersion using $0.02 \mathrm{wt} \%$ PVA at 120 minutes.

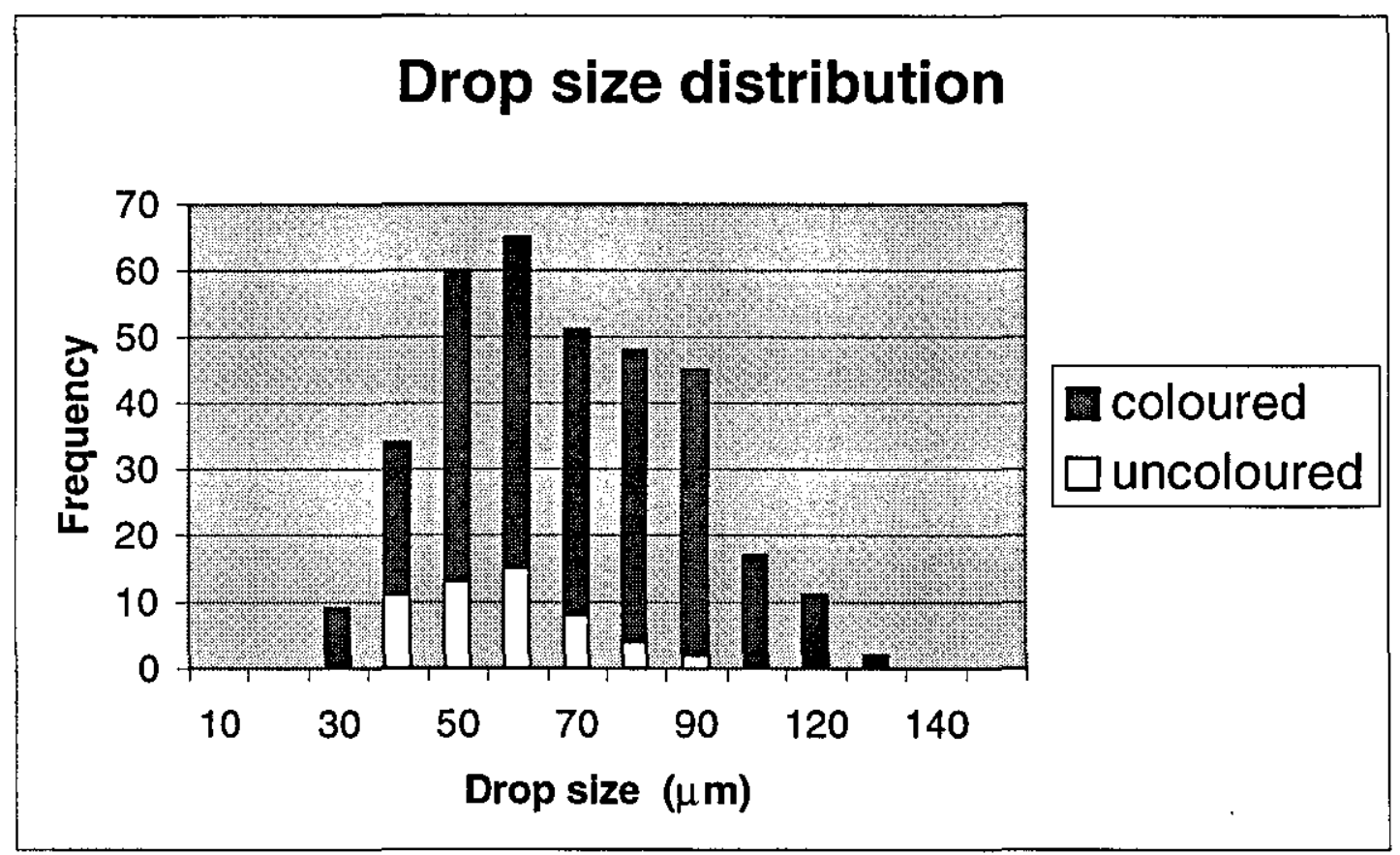

Figure 4.3b : Drop size distribution for mixing of dispersion without stabiliser and dispersion using $0.02 \mathrm{wt} \%$ Tween 20 at 120 minutes. 


\subsubsection{Extent of mixing for two different conditions using 0.02 wt \% Tween 20}

In these experiments, two sets of mixing conditions were used for comparison. The first experiment was the mixing of two dispersions of toluene in $0.02 \mathrm{wt} \%$ Tween 20, in which one of the dispersions was coloured. For the second experiment, the mixing was done between a dispersion of toluene without stabiliser and a dispersion of toluene (coloured) in 0.02 wt \% Tween 20.

Figure 4.4 shows the results of using two different conditions of mixing for $0.02 \mathrm{wt} \%$ Tween 20 . The results show that the extent of mixing for the mixing of a dispersion of toluene without stabiliser was higher than that with $0.02 \mathrm{wt} \%$ Tween 20. This is because the dispersion of toluene without stabiliser was not protected with surfactant; therefore their mixing rates were higher compared to the mixing of the dispersions with surfactant. The slower mixing rate between the two stabilised dispersions can be explained by the fact that all the drops were stabilised by the Tween 20. It also shows that although all the drops were covered and stabilised by surfactant, coalescence can still occur. These experiment show that breakage and coalescence continuously occur during the dispersion. 


\section{Extent of mixing for two different conditions}

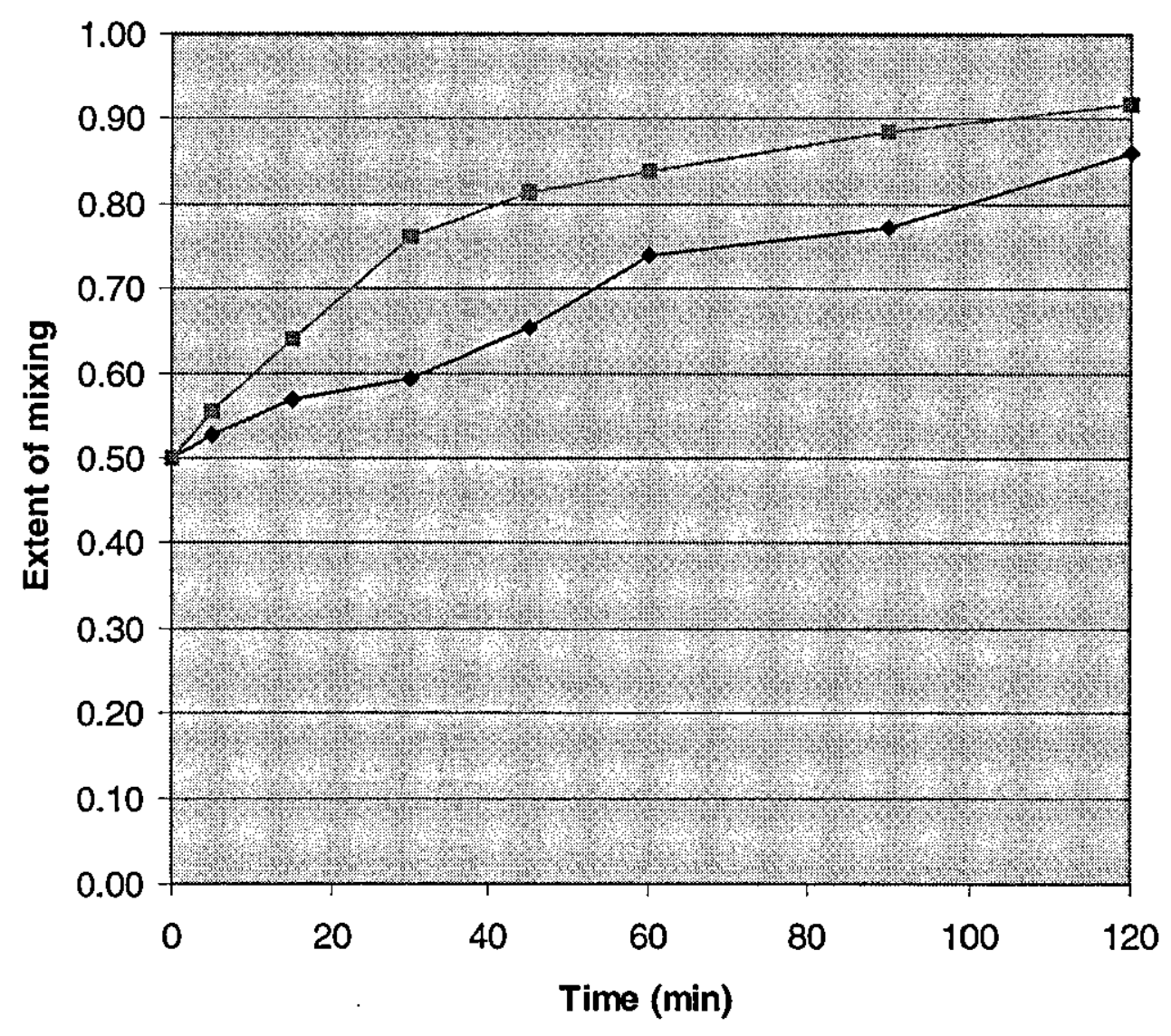

$\rightarrow-$ Exp1 - Dispersion of toluene using $0.02 \%$ Tween 20 and dispersion of toluene using $0.02 \mathrm{wt} \%$ Tween 20

-Exp2 - Dispersion of toluene without stabiliser and dispersion of toluene using 0.02 wt \% Tween 20

Figure 4.4: Extent of mixing for two different condition of mixing 


\subsection{Batch mixing of styrene with stabilised dispersion of polystyrene solution}

The preliminary experiments have confirmed the suitability of the equipment and the technique to determine the extent of mixing in the drop mixing studies. For the detailed study of the drop mixing, styrene was used instead of toluene. That would simulate the suspension process for styrene polymerisation. The technique of photographing the sample was also changed to using a video system. The video system seemed to have produced more clear images to compare the coloured and uncoloured drops. It also reduced the time of analysing the data. However on the detailed examination of the images produced via the microscope, one limitation arose. It was found that the drop colour paled with decreased in drop size. There was no significant different between coloured and uncoloured drops if the drops are too small. From the detailed observation, it was found that drops below $5 \mu \mathrm{m}$ are very difficult to compare. All the drops seemed uncoloured even though some drops contained dye. For this reason, all experiments have to be designed so that no drops or only a negligible amount of drops below $5 \mu \mathrm{m}$ are present in the dispersion. Large quantity of drops below $5 \mu \mathrm{m}$ may lead to significant error in the analysis of the drop mixing. Furthermore too many large drops also may produce a significant error because the whole parts of the drops cannot fit into the image screen.

\subsubsection{Dispersion behaviour of the dispersed phase before mixing of new batch}

The purpose of the experiments was to observe the behavior of the liquidliquid dispersion before the addition of a new batch. Therefore comparison can be made between drop behavior before and after the addition of new styrene. Three different viscosities of polystyrene solution were used to study the behaviour of the liquid dispersion, they were $5 \mathrm{wt} \%, 10 \mathrm{wt} \%$ and $15 \mathrm{wt} \%$ of polystyrene solution. The dispersion experiments were run using the following experimental conditions:

$\begin{array}{ll}\text { Dispersed phase volume } & 40 \mathrm{ml} \\ \text { Continuous phase volume } & 450 \mathrm{ml} \\ \text { Temperature } & 30^{\circ} \mathrm{C} \\ \text { Suspending agent } & 0.02 \mathrm{wt} \% \text { PVA }\end{array}$


The experiments were run for 150 minutes and, at each stirring time of $5,15,30,45$, $60,90,120$ and 150 minutes, the image of the drops were taken for the analysis.

In order to consider the flow field of the dispersion to be locally isotropic, the impeller Reynold numbers, Re (equation [2.3]), should be larger than $10^{4}$ (Rushton, J.H. et al 1950). In this experimental condition, $N=500 \mathrm{rpm}$ and $\mathrm{D}=10 \mathrm{~cm}$ while the value for $v$ depends on the viscosity of the dispersed phase. Table 4.1 shows the value of $v$ and impeller Reynold number for the dispersions using the three different viscosities of dispersed phase, repectively. All the Reynold number for the dispersions are well above the minimum value for the local isotropy theory to be valid.

Table 4.1: Reynold number for different viscosities of dispersed phase.

\begin{tabular}{|c|c|c|}
\hline Polystyrene Solution & Kinematic viscosity $(\mathrm{v}) / \mathrm{cm}^{2} / \mathrm{s}$ & Reynold Number \\
\hline $5 \mathrm{wt} \%$ & 0.0102 & 82000 \\
\hline $10 \mathrm{wt} \%$ & 0.0119 & 69800 \\
\hline $15 \mathrm{wt} \%$ & 0.0120 & 69700 \\
\hline
\end{tabular}

Figure 4.5 shows the mean drop size as a function of stirring time for three different viscosities of dispersed phase. The drop size for the three dispersions decreased with time until it remain more or less constant. These profiles are in agreement with the work done by Hong P.O. and Lee J.M (1983 and 1985) and. Zerfa, M and Brooks, B.W. (1996a). During the initial period of liquid-liquid dispersion, the breakage rate dominates the coalescence rate. The results show that the dynamic equilibrium for the three dispersions in the $0.02 \mathrm{wt} \%$ PVA was achieved after about 60 minutes. At this stage, a steady state is established during which the breakage rate is equal to the coalescence rate. 


\section{Mean Drop Size}

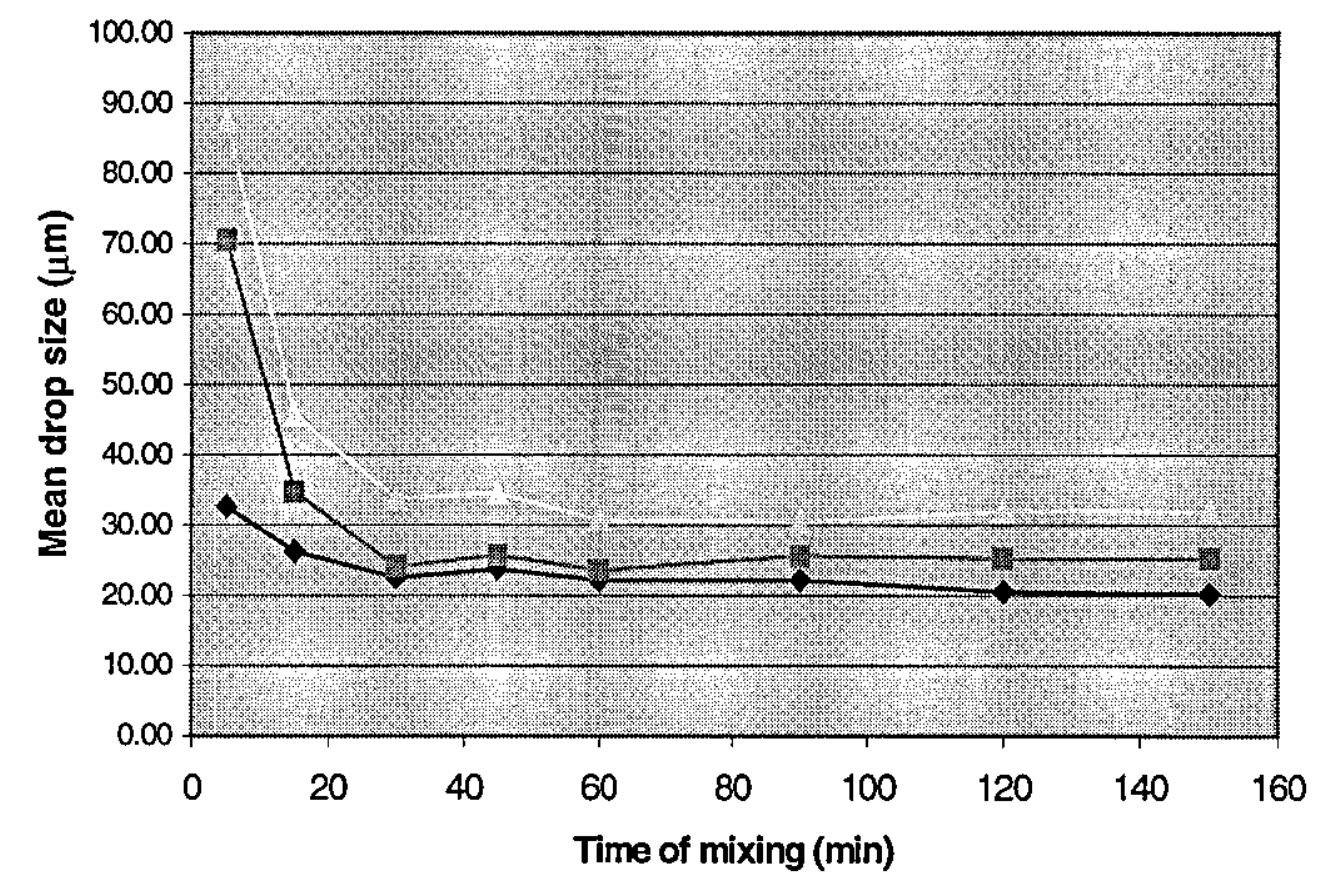

$$
\begin{aligned}
& --5 w t \% P S \\
& --10 w t \% P S \\
& -\rightarrow-15 w t \% P S
\end{aligned}
$$

Figure 4.5: Mean drop size as function of stirring time for different polystyrene solution 
The slow decrease toward steady state can be explained because of the time taken for PVA molecules to absorb on the droplet interface, rearrange themselves and protect the droplet against coalescence. It also shows that, with higher dispersed phase viscosity, a larger average drop size was produced. Similar results were obtained by Wang, C.Y. and Calabrese R.V. (1986), Kuriyama, M. et al (1996) and Arai, K. et al. (1977) on their study on the effect of viscosity in liquid-liquid dispersion. The viscosity of the dispersed phase has contributed additional cohesive force, therefore the final size of drops were greater due to less break up. It can also be seen that from the results, at 120 minutes, the dispersed phase drop has already reached the steady state. The time of 120 minutes was the time at which the new batch of styrene will be added to an existing dispersion in the next experiments of batch addition of styrene to polystyrene dispersion.

Figure 4.6 shows the drop size distribution of the three dispersions at 120 minutes. The results show that as the dispersed phase viscosity increased, the drop size distribution broadens. Drop size and drop size distribution are important parameters that can affect the coalescence rate between drops.

\subsubsection{Drop mixing between a new batch of styrene and the existing stabilised dispersion using $0.02 \%$ PVA}

In these experiments, a batch of dyed styrene was added to a stabilised polystyrene solution of a different viscosity. The objective was to study the effects of different viscosity on the mixing rate between the added batch and the existing stabilised drops. The stabilised dispersions were prepared and run as in section 4.2.1. At 120 minutes, $10 \mathrm{ml}$ of styrene with $1 \mathrm{~g}$ of dye was introduced to the dispersion. Then the samples were taken at $5,15,30,45,60,90,120,150$ and 180 minutes and their images were obtained for further drop size analysis. Extent of mixing was then determined as a function of time. The experiments were run at different stirring speeds. The stirring speed will affect the turbulent intensity and the drop size of the dispersion. Experiments on the reproducibility of the results were also done and these are discussed in detail in appendix D. The variation of the extent of mixing was found to be \pm 0.025 . 


\section{DROP SIZE DISTRIBUTION}
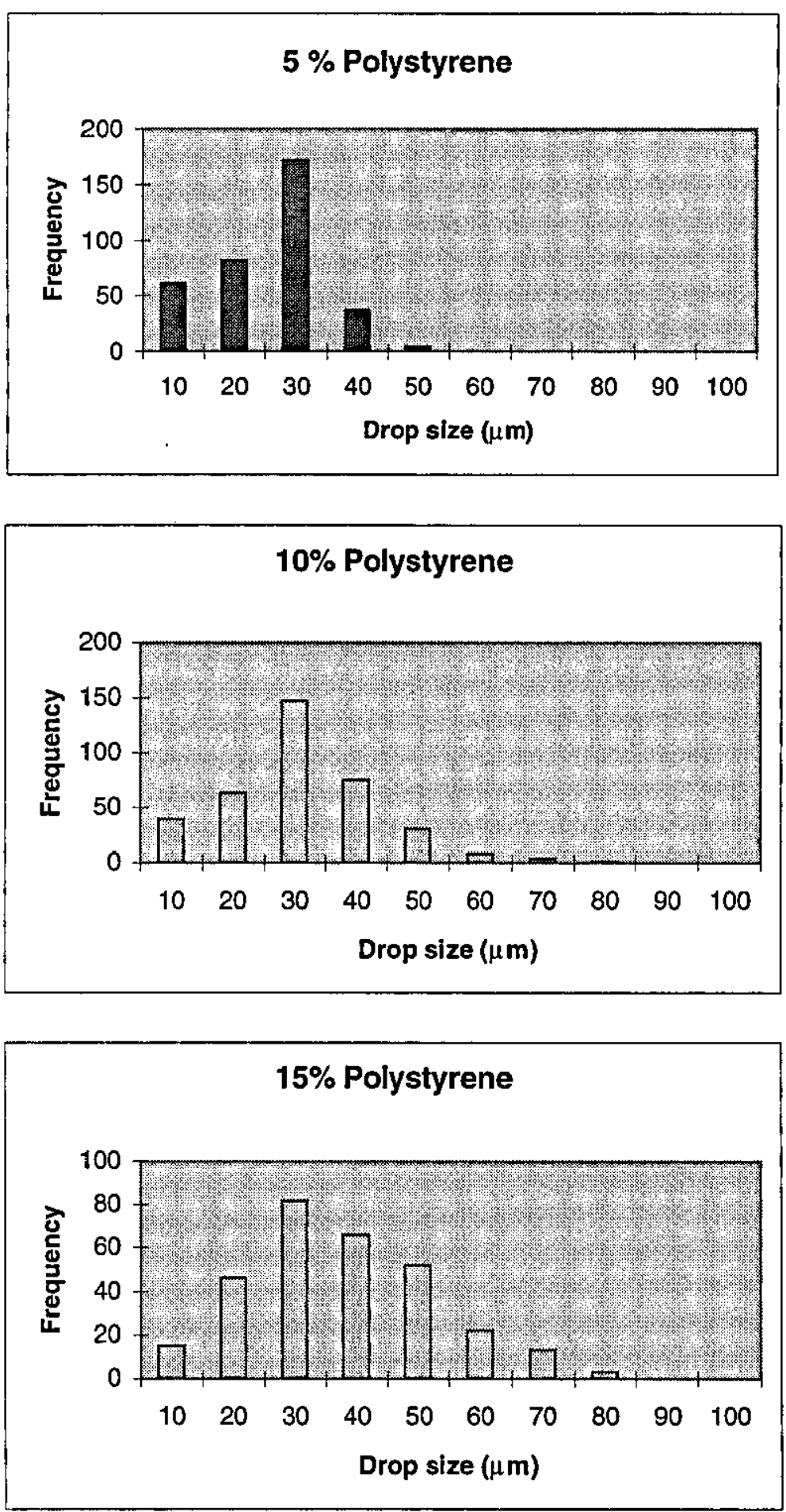

Figure 4.6: Drop size distribution of different polystyrene solution at 120 minutes 


\subsubsection{Drop mixing at $500 \mathrm{rpm}$}

In this section, the series of mixing experiments were run at $500 \mathrm{rpm}$. Four concentrations of dispersed phase were used, $5 \mathrm{wt} \%, 10 \mathrm{wt} \%, 15 \mathrm{wt} \%$ and $20 \mathrm{wt}$ $\%$ of polystyrene solution, respectively. Each concentration has its own characteristic viscosity as shown in chapter 3. Figure 4.7 shows the images from drop mixing between coloured and uncoloured drops at different stirring times. The extent of mixing as a function of stirring time, for the different viscosities of polystyrene solution, are shown in figure 4.8. The result from the $5 \mathrm{wt} \%$ of polystyrene solution is not included because the result seemed to have a significant error. The error was noticed after detailed observation, it revealed that there were a large amount of drops that were below $5 \mu \mathrm{m}$. As explained before, drops below $5 \mu \mathrm{m}$ will cause an error in distinguishing coloured and uncoloured drops. Therefore this result was omitted from the discussion.

Figure 4.8 shows that viscosity influenced the mixing rate between the new batch and the existing dispersion. It can be seen that as the dispersed phase viscosity increased, the mixing rate increased. The results contradicted the model proposed by Ivanov, I.B. and Traykor, T.T. (1976) and Chesters (1991). They proposed that the drainage rate will be slower with the increase viscosity of the dispersed phase. Therefore coalescence efficiency will be lower. Kumar, S. et al (1993) and Kuriyama, M. et al (1996) also proposed that as the dispersed phase viscosity increased, the pertubation of the drops will decrease and that will cause the coalescence efficiency to decrease. To explain this behavior of drop mixing in these experiments, further observation was made on the analysis of the drop size. As noticed before, the drop size before the addition of new batch depends on the viscosity of the drops. The size of the drops increases as the viscosity increases. An increase in drop size may cause the squeezing force and time of contact between drops to increase. As a result the coalescence efficiency is increased.

Figure 4.9 shows the comparison between the drop size distribution for the dispersed phase of $10 \mathrm{wt} \%$ with that for $15 \mathrm{wt} \%$ polystyrene solution at 180 minutes. The results show that the $15 \mathrm{wt} \%$ polystyrene solution dispersed phase gives a higher 


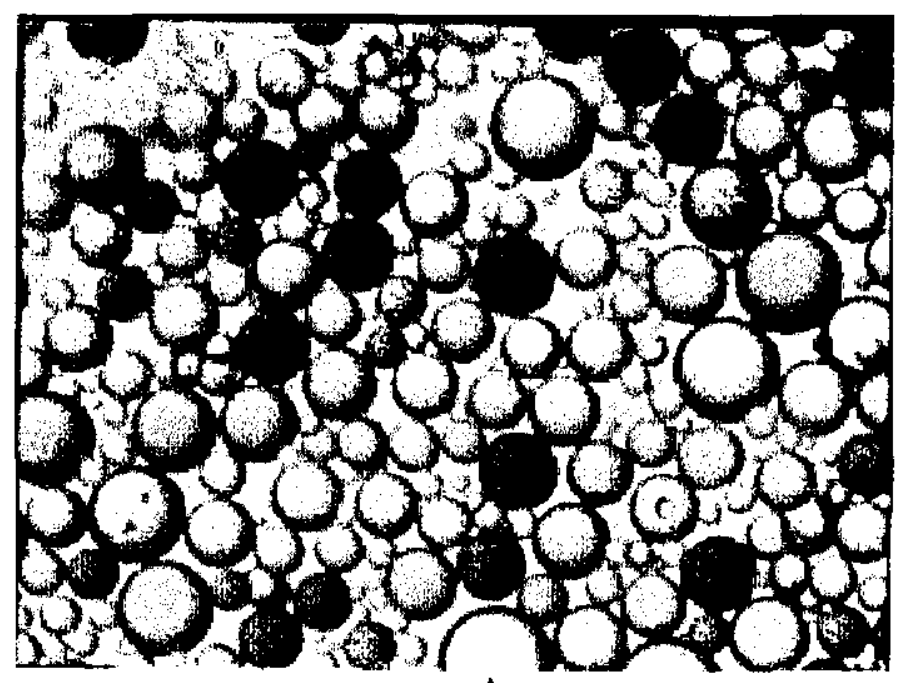

A
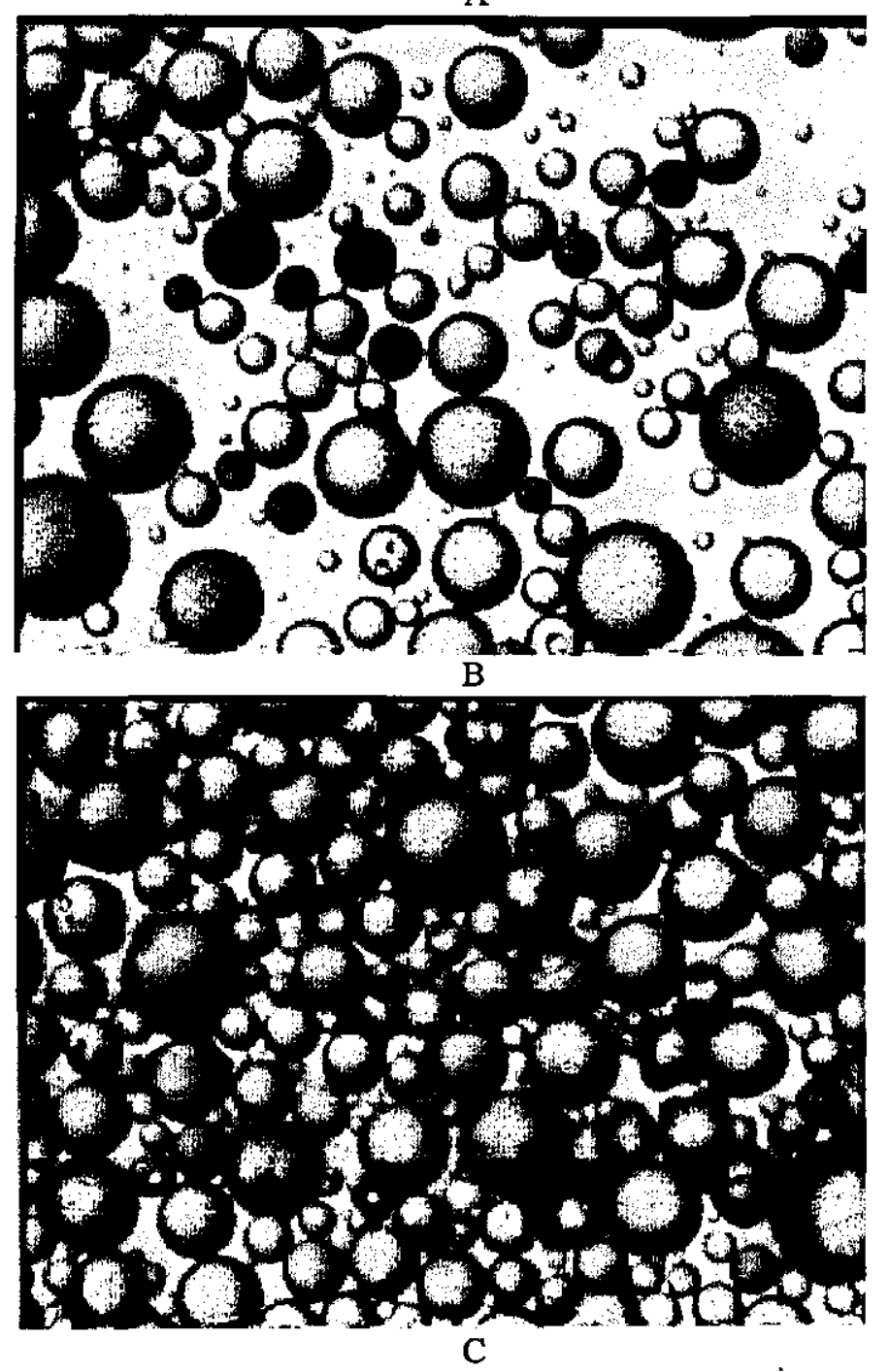

Figure 4.7: Drop mixing between coloured and uncoloured drops for 15 wt $\%$ polystyrene solution at 3 different stirring times: A. 15 minutes B. 60 minutes C. 120 minutes. 


\section{Extent of mixing for batch mixing at $500 \mathrm{rpm}$}
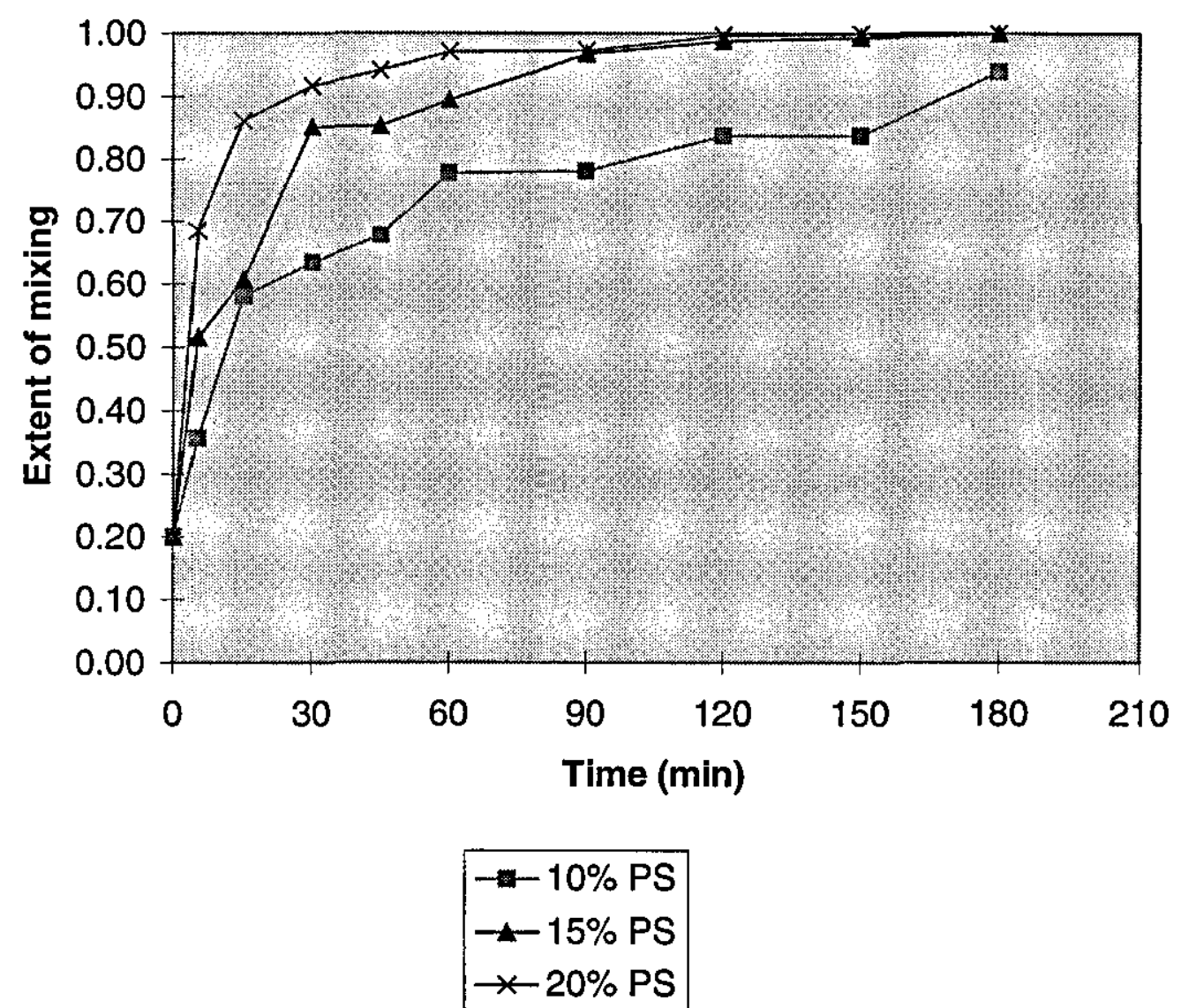

Figure 4.8: Extent of mixing for batch mixing of new styrene with different viscosities of dispersed phase 

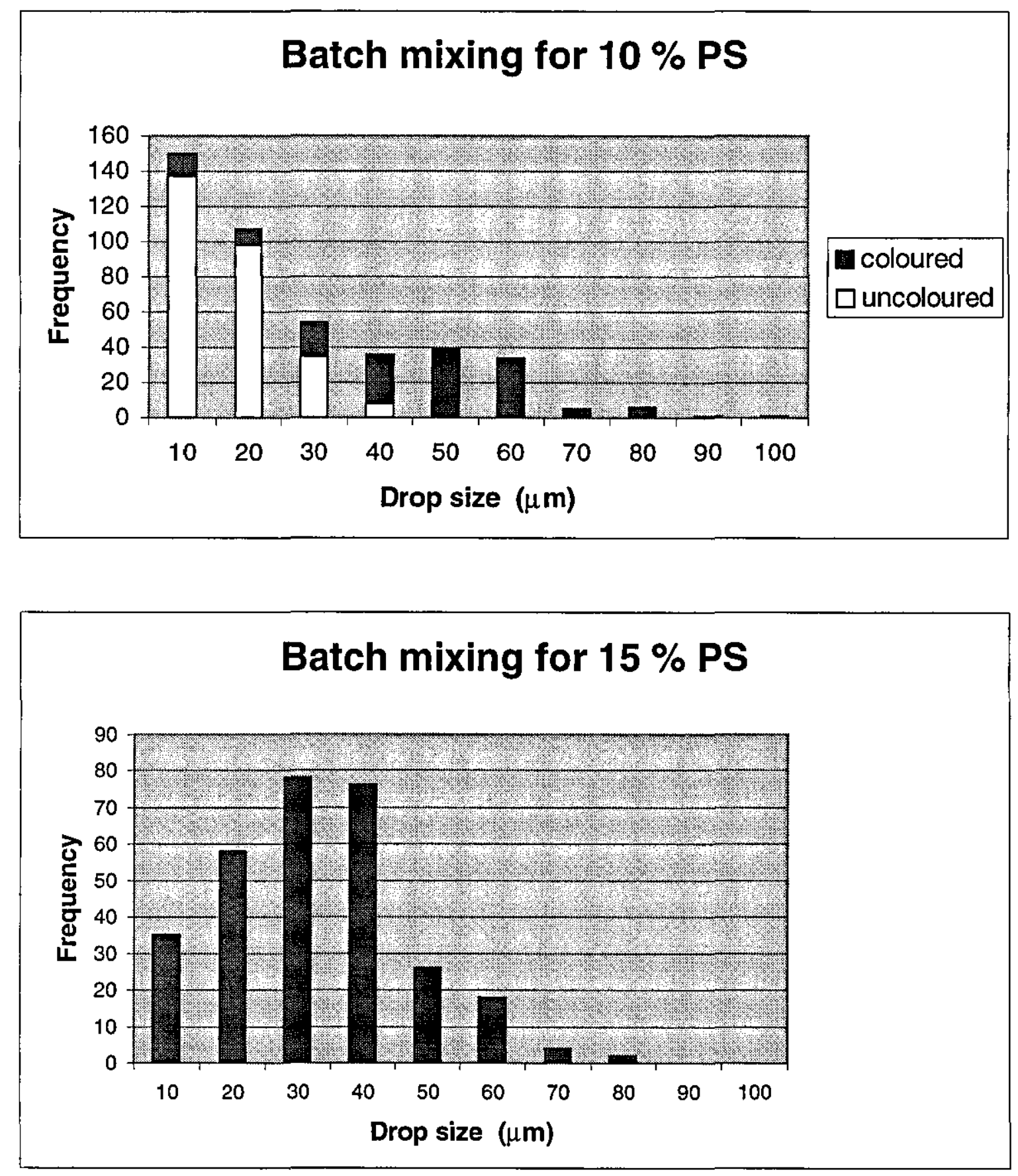

Figure 4.9: Drop size distribution for batch mixing at 180 minutes. 
mean drop size and wider mean drop size distribution compared to $10 \mathrm{wt} \%$ polystyrene solution of dispersed phase. The figures also show that for $10 \mathrm{wt} \%$ polystyrene solution dispersed phase, not all drops are coloured, however it can be seen that all larger drop size are coloured. In these conditions it can be said that coalescence occurs more efficiently at larger drop size. It appears that small droplets are relatively stable towards coalescence. Therefore the rate of coalescence for smaller droplets is lower.

Therefore in this experimental condition it can be said that drop size plays an important role in determining the extent of mixing between drops, the mixing rate increased as the drop size increased. Tobin, T. et al (1990) also had the similar findings, in their study of the purely coalescing dispersions. They found that coalescence frequency is an increasing function of drop size. Visual observations of coalescence by Park, J.Y. and Blair, L.M. (1975) also led them to believe that the efficiencies increase with drop size.

From the above observation and as shown in figure 4.8 , the mixing rate between higher viscosity of dispersed phase and a new added batch of styrene is higher due to the larger drop size in the dispersion. Therefore at this experimental condition the viscosity affects the coalescence efficiency indirectly.

Figure 4.10 shows the transitional behavior of drop size distribution for the mixing at 20 wt \% polystyrene solution of dispersed phase with styrene. The result shows that at 5 minutes all the larger drops are coloured, then gradually the smaller drops become coloured until all the drops become coloured at 180 minutes. These transitional behavior also shows that larger drops have higher coalescence efficiency compared to the smaller drops. Therefore it can be said that the new batch of styrene mixed with larger drops and followed by smaller drops mixed with coloured drops. The figure also shows that the drop size distribution starts to shift to the left gradually from the beginning of the mixing with the decreasing maximum drop size. This phenomena can be explained by suggesting that as the mixing between drops of high viscosity with the new batch styrene occurs, the new drops that are formed will have a lower viscosity than before. Therefore with lower viscosity the overall drop size of the droplets will decrease. Also during the dispersion the breakage and coalescence 

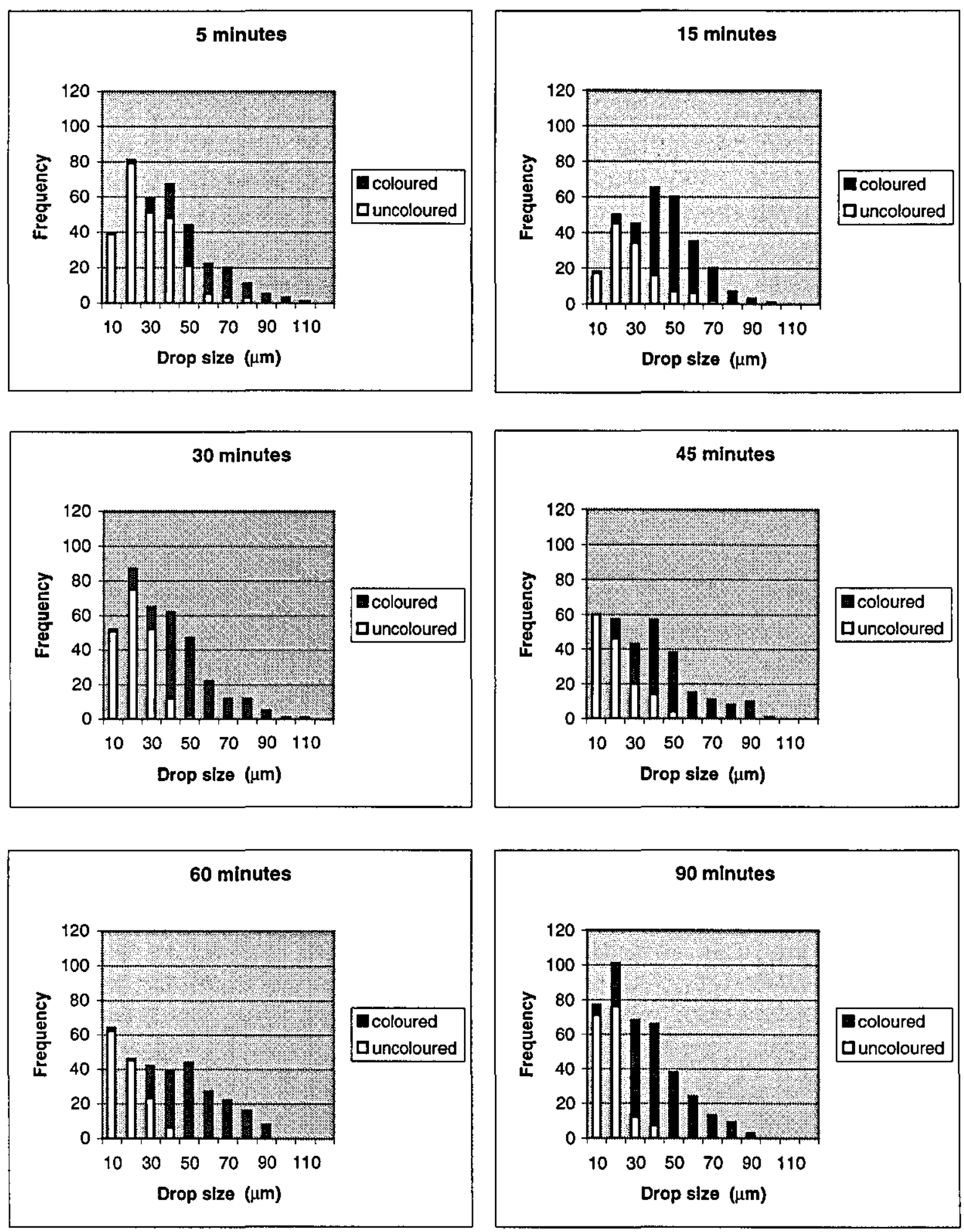

Figure 4.10: Drop size distribution for batch mixing of styrene with 20 wt $\%$ PS dispersed phase

(continue on next page) 

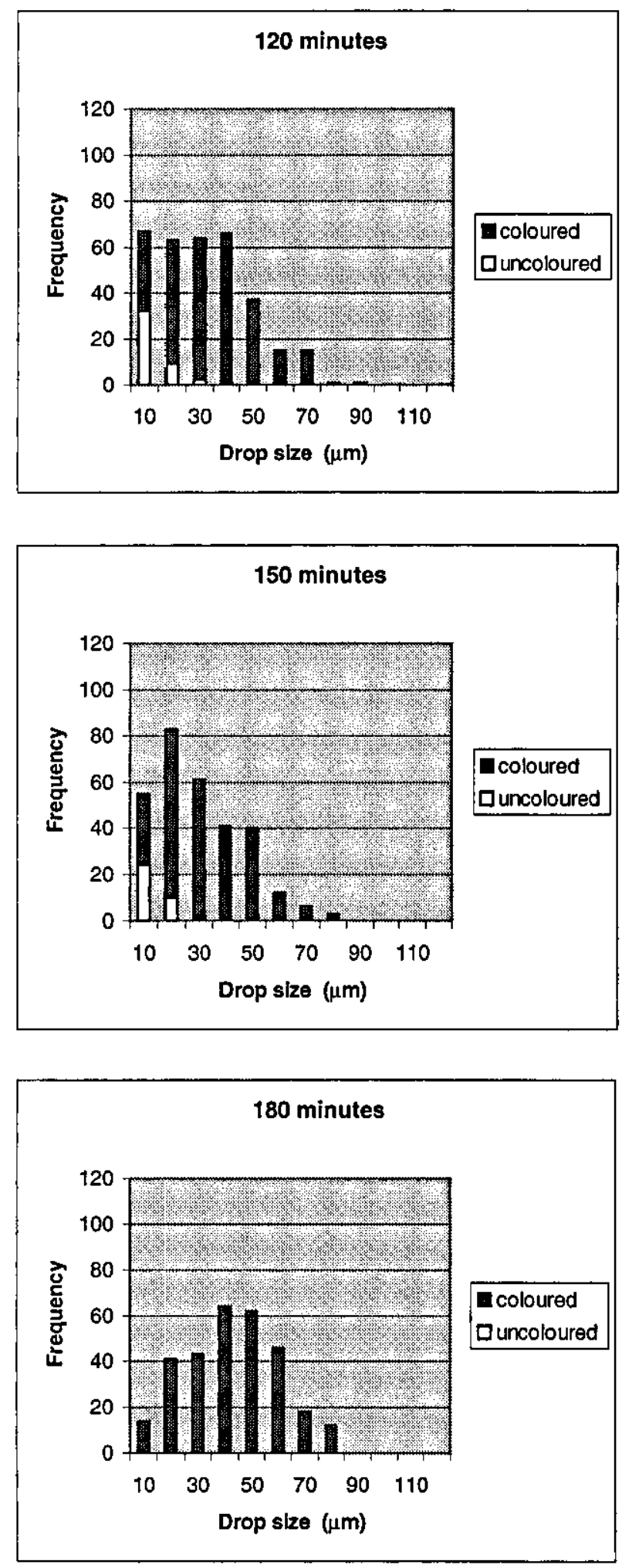

Figure 4.10 (continued) 
of the drops are occuring simultanouesly. The coalescence not only occurs between coloured and uncoloured drops but it can also occur between solely coloured drops and also between solely uncoloured drops. It can also be seen that the drop size distribution becomes a normal distribution when all drops are coloured. At this stage the drop size distribution can reach the steady state.

Figure 4.11 shows the mean drop size for coloured and uncoloured drops at 15 and 20 wt $\%$ polystyrene solution dispersed phase as a function stirring time. As can been seen from the figure, mean drop size for coloured and uncoloured drops for both viscosities of dispersed phase decreases gradually as a function of stirring time. It appears that the mean drop size for coloured drops decreases because of decreasing drop viscosity due to the mixing between the high viscosity dispersed phase and the added styrene. While for uncoloured drops, the mean drop size decreases due to the loss of larger drops that mixes with the coloured drops. Therefore the mean drop size of uncoloured drops decreases as only smaller drops are available toward the end.

\subsubsection{Drop mixing at $650 \mathrm{rpm}$}

These experiments were run at the same conditions as in the previous section except a higher stirrer speed, $650 \mathrm{rpm}$ was used. The purpose of these experiments was to observe the drop mixing behaviour between a new batch of styrene and the existing dispersed phase at different speeds. Since higher stirrer speed caused lower drop size, the mixing experiment for $5 \mathrm{wt} \%$ and $10 \mathrm{wt} \%$ polystyrene solution dispersed phase results in a very poor image when distinguishing coloured and uncoloured drops. Therefore the experiments were run only for $15 \mathrm{wt} \%$ and $20 \mathrm{wt} \%$ polystyrene solution dispersed phase. Figure 4.12 show the extent of mixing as a function of stirring time for $15 \mathrm{wt} \%$ and $20 \mathrm{wt} \%$ polystyrene solution dispersed phase at 650 rpm. The results indicate that the drop mixing occurs very fast at this stirring speed with larger viscosity mixing slightly faster compared to lower viscosity. With the same explanation as described in the previous section, drop size plays an important role in this mixing. Due to larger drop size in the dispersion of $20 \mathrm{wt} \%$ polystyrene solution disperse phase, the rate of mixing between coloured and uncoloured drops is higher. The higher viscosity has caused the drop size to be larger. 

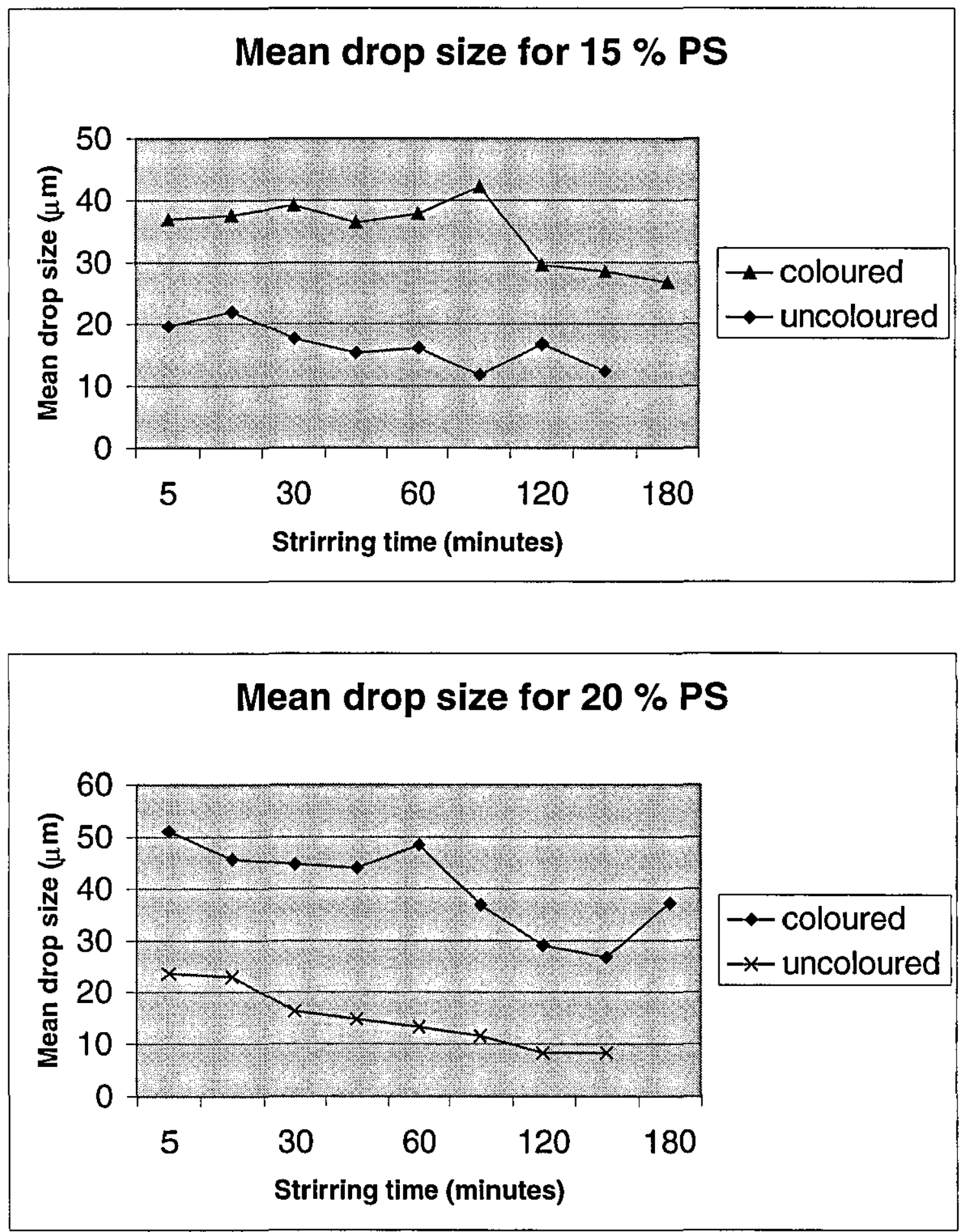

Figure 4.11: Mean drop size for coloured and uncoloured drops at $15 \mathrm{wt} \%$ and 20 wt \% PS dispersed phase at $500 \mathrm{rpm}$ 


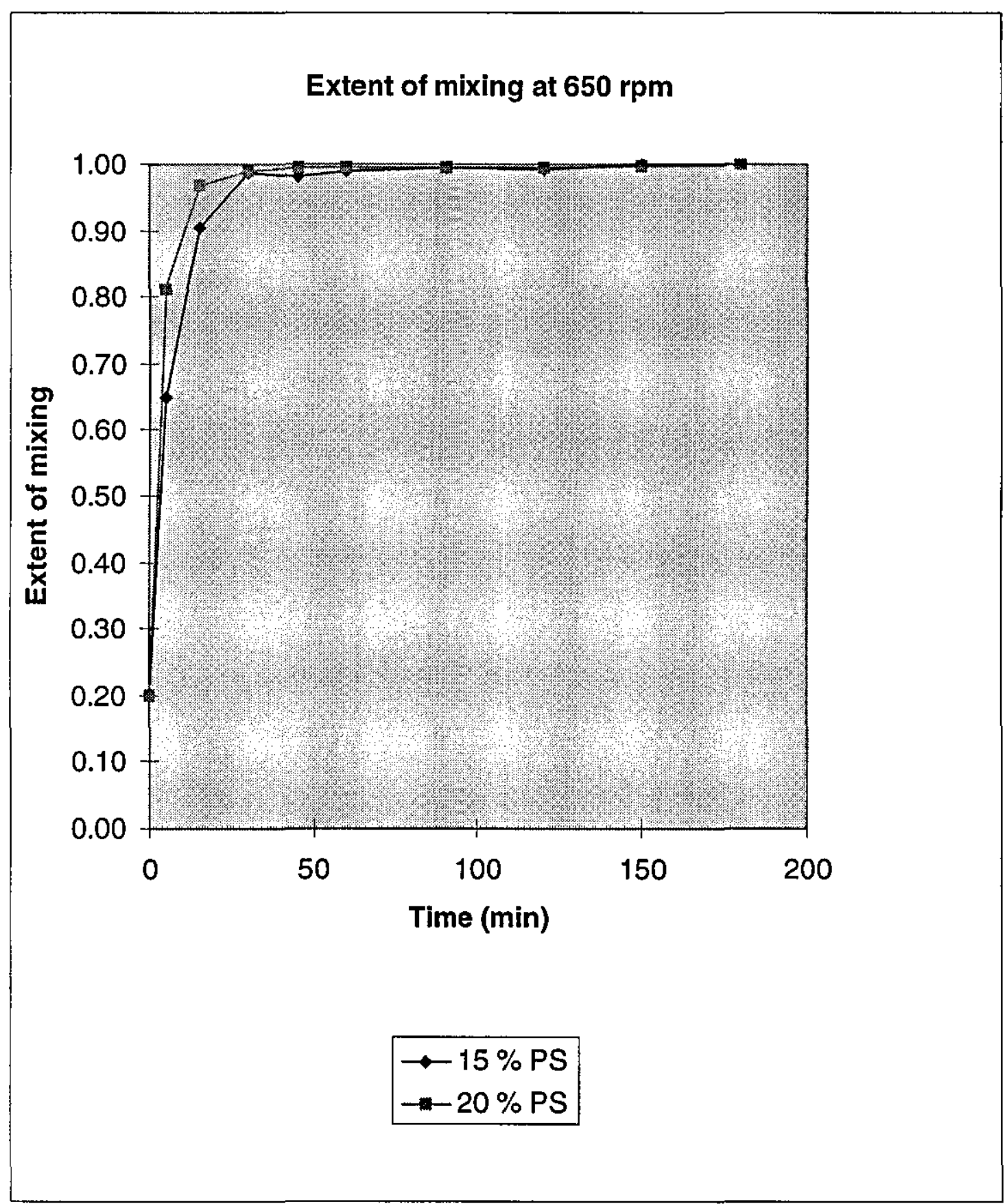

Figure 4.12: Extent of mixing for batch mixing of new styrene with different viscosities of dispersed phase at $650 \mathrm{rpm}$ 
Figure 4.13 shows the transitional behavior of drops size distribution for batch mixing of styrene and $15 \mathrm{wt} \%$ polystyrene solution dispersed phase at $650 \mathrm{rpm}$. It can be seen that mixing with coloured drops occur at larger drop size then followed by lower drop size. This mixing behaviour is the same as in the mixing at $500 \mathrm{rpm}$. Again, these results confirm the previous suggestion that coalescence rate is an increasing function of drop size.

\subsubsection{Drop mixing at $350 \mathrm{rpm}$}

The purpose of these experiments was to observe the drop mixing behaviour between a new batch of styrene and the existing dispersed phase at a lower stirring speed. These experiments were run at the same conditions as in the previous section except running at $350 \mathrm{rpm}$. Since lower stirring speed results in larger drop size, the mixing experiment could be successfully run and analysed for a dispersed phase of 5 wt $\%, 10 \mathrm{wt} \%$ and $15 \mathrm{wt} \%$ polystyrene solution. The extent of mixing as a function stirring time for the mixing between a new batch and existing dispersed phase at $\mathbf{3 5 0}$ $\mathrm{rpm}$ is shown in figure 4.14. In order to show that the variation of extent of mixing as function of viscosity in figure 4.14 is real, error bars were plotted on of the data series (5 wt \% PS). As expected, the extent of mixing increases relatively slowly compared to the batch mixing at higher stirring speed. It can be seen from the results that the mixing occured more slowly with the higher dispersed phase viscosity. These results contradicted the mixing experiments that were run at $500 \mathrm{rpm}$ and $650 \mathrm{rpm}$. However these results were consistent with the model proposed by Ivanov and Traykor, I.B. (1976) and Chesters, A.K. (1991). An increase in drop phase viscosity will result in a slower drainage rate. Kumar S. (1993) observed that, at larger drop size, as the drop deformation becomes significant it leads to the formation of a disc of contact and results in the decrease in drainage rate. Therefore at this larger drop size, with increasing drop viscosity the coalescence efficiency become lower. Comparing with the result of batch mixing studies at $500 \mathrm{rpm}$ and $650 \mathrm{rpm}$ previously, it can be said that there is a critical drop size, which determines the coalescence efficiency effect. Above the critical drop size, mixing rate increases as the drop viscosity decreases. While below the critical drop size, drop size plays an important role in determining the mixing rate; as the drop size increased, mixing rate will also increase. However it is very difficult to determine the critical drop size and that is beyond the scope of this study. 

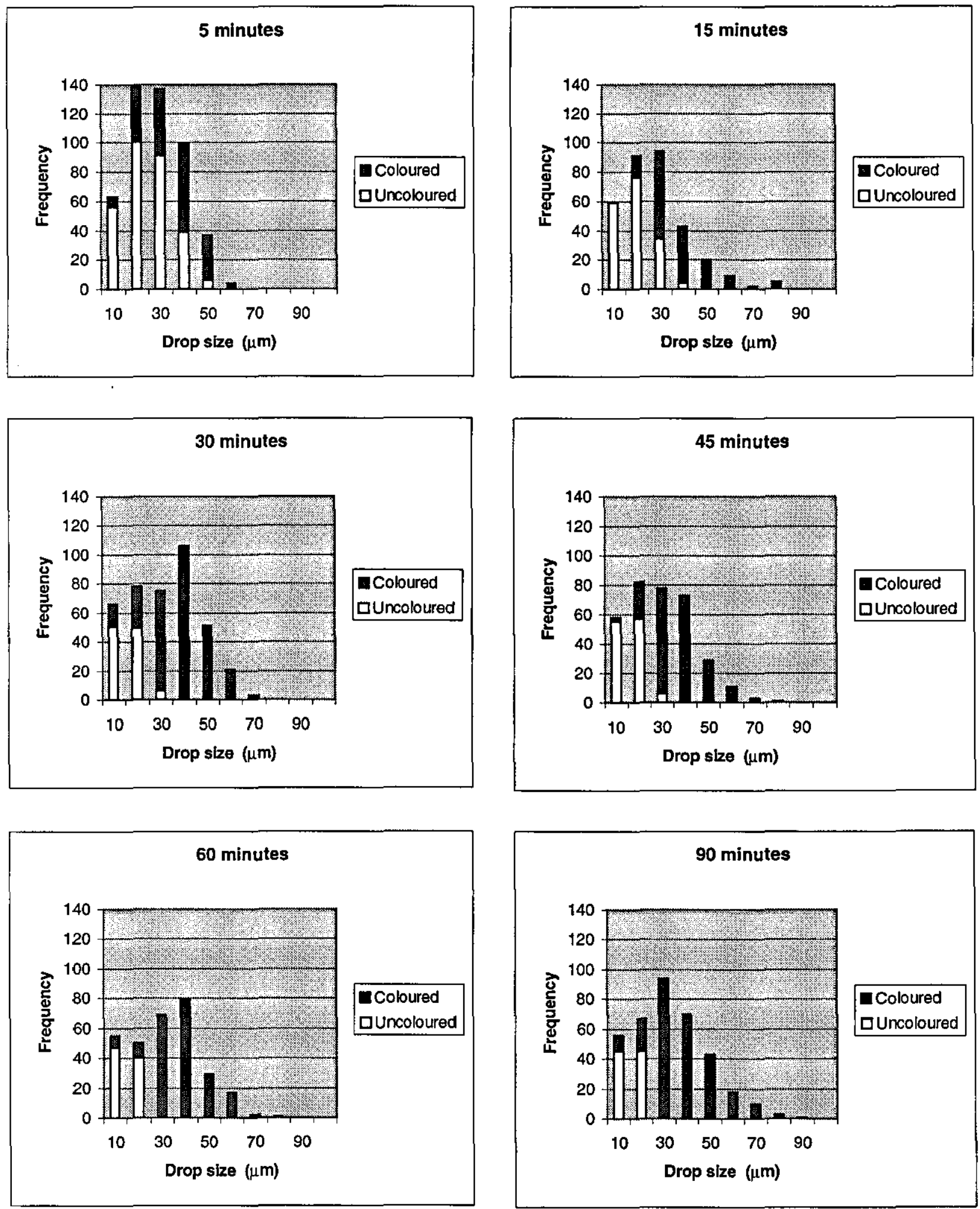

Figure 4.13: Drop size distribution for batch mixing of styrene with $15 \mathrm{wt} \%$ PS dispersed phase at $650 \mathrm{rpm}$

(continue on next page) 

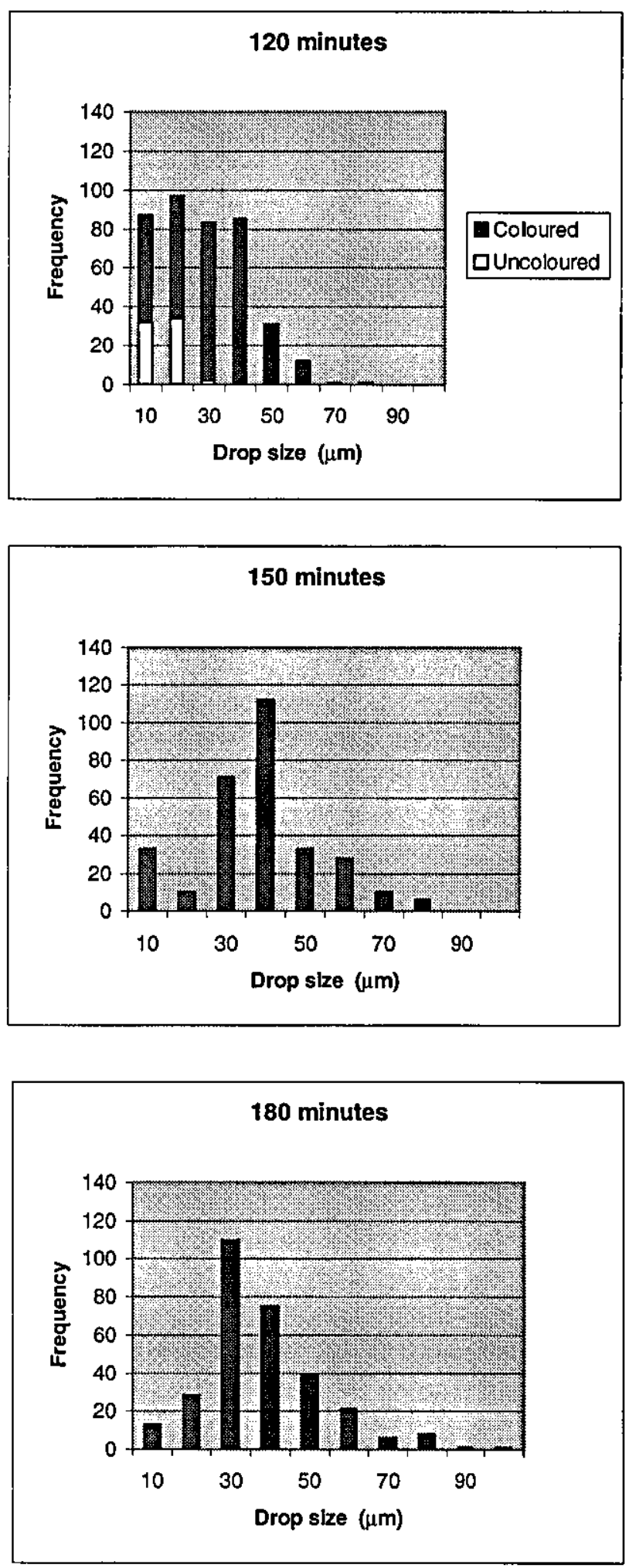

Figure 4.13 (continued) 


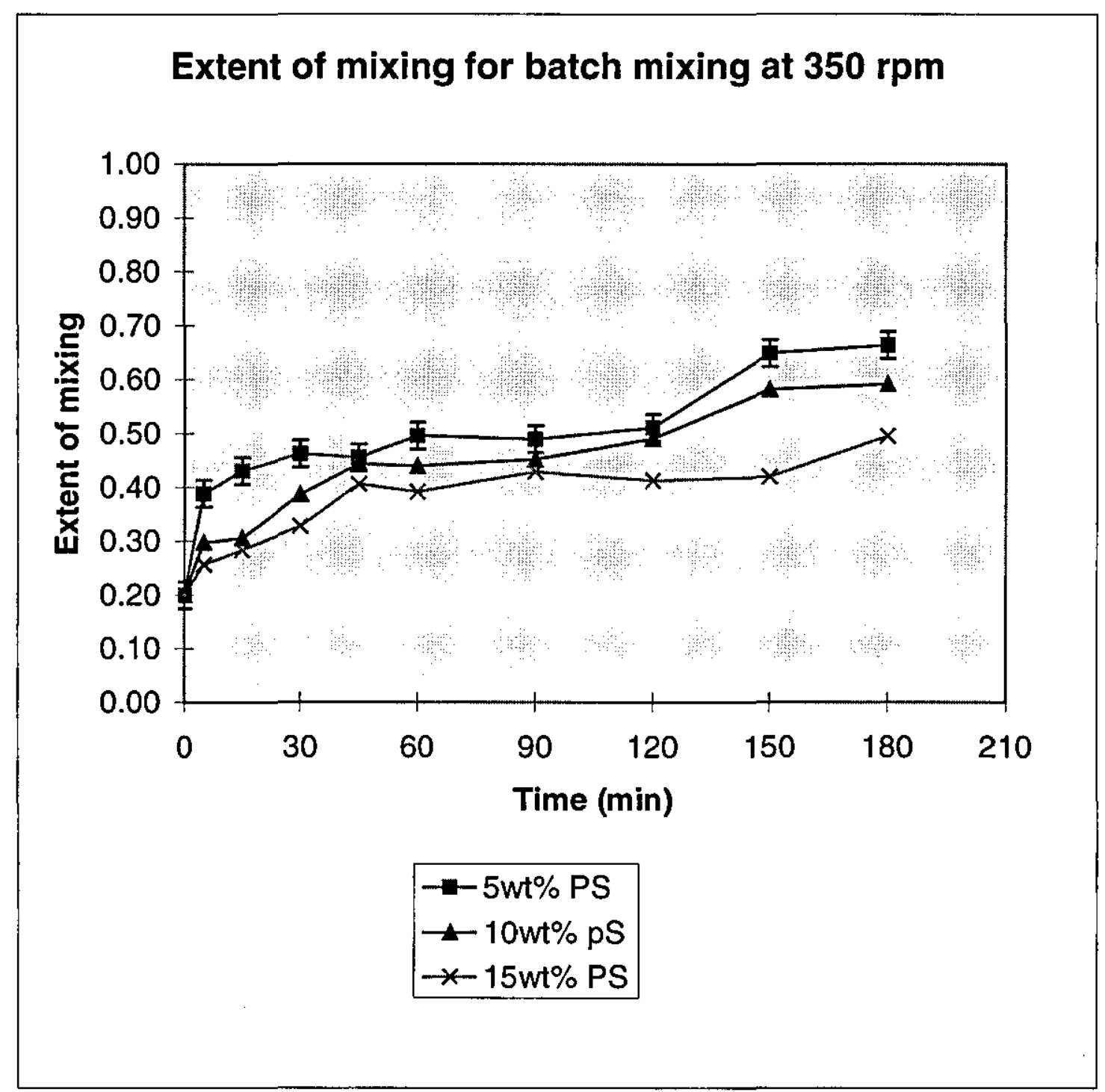

Figure 4.14: Extent of mixing for batch mixing of new styrene with different viscosities of dispersed phase at $350 \mathrm{rpm}$. 
The transitional behavior of drop size distribution for the mixing of styrene with $15 \mathrm{wt}$ $\%$ polystyrene solution of dispersed phase is shown in figure 4.15. The result shows that at five minutes, coloured drops mixed mostly at the middle range of the drop size. The larger size of uncoloured drops seemed to be very stable toward coalescence. The mixing continued to occur mostly in the middle range of the drop size until at 180 minutes. This result is different from the batch mixing studies at $500 \mathrm{rpm}$ and 650 rpm where drop mixing between coloured and coloured drops starts from larger drop size followed by lower drop size. However the large drops in the mixing studies at $500 \mathrm{rpm}$ and $650 \mathrm{rpm}$ are in the range equivalent to middle range of drop sizes in the mixing studies at $350 \mathrm{rpm}$. Therefore these results proved the existence of a critical drop size. For the larger drop size, viscosity effects play an important role in determining the coalescence efficiency.

\subsubsection{Effect of stirring speed on batch mixing}

The purpose of this subsection is to compare the behaviour of batch drop mixing at different stirring speeds. Figure 4.16 shows the extent of mixing for batch mixing of styrene to $15 \mathrm{wt} \%$ polystyrene solution at $350 \mathrm{rpm}, 500 \mathrm{rpm}$ and $650 \mathrm{rpm}$. It can be seen that the higher the stirring speed the higher the mixing rate between the coloured and uncoloured drops. For the stirring speed of $500 \mathrm{rpm}$ and $650 \mathrm{rpm}$, the mixing rate is relatively high, all droplets become almost coloured after 120 minutes. While for the lower stirring speed of $350 \mathrm{rpm}$, the mixing rate is quite low. The extent of mixing of the coloured drops is still under $50 \%$ at 180 minutes after the start of mixing with the new batch styrene.

The comparison was also done on the drop size distribution at a certain stirring time. Figure 4.17 shows the drop size distribution at 5 minutes for $15 \mathrm{wt} \%$ polystyrene solution dispersed phase at different stirring speeds. The results show that at lower stirring speed, the drop sizes are bigger and have wider drop size distribution compared to the higher stirring speed. It can also be seen that most larger drops are coloured at the stirring speed of $500 \mathrm{rpm}$ and $650 \mathrm{rpm}$. While for the mixing at 350 rpm, larger drops size are still stable toward coalescence. It should be noted that even though the percentage of uncoloured drops seemed to higher for $500 \mathrm{rpm}$ than 350 rpm, the extent of mixing was measured based on the volume of the drops. 

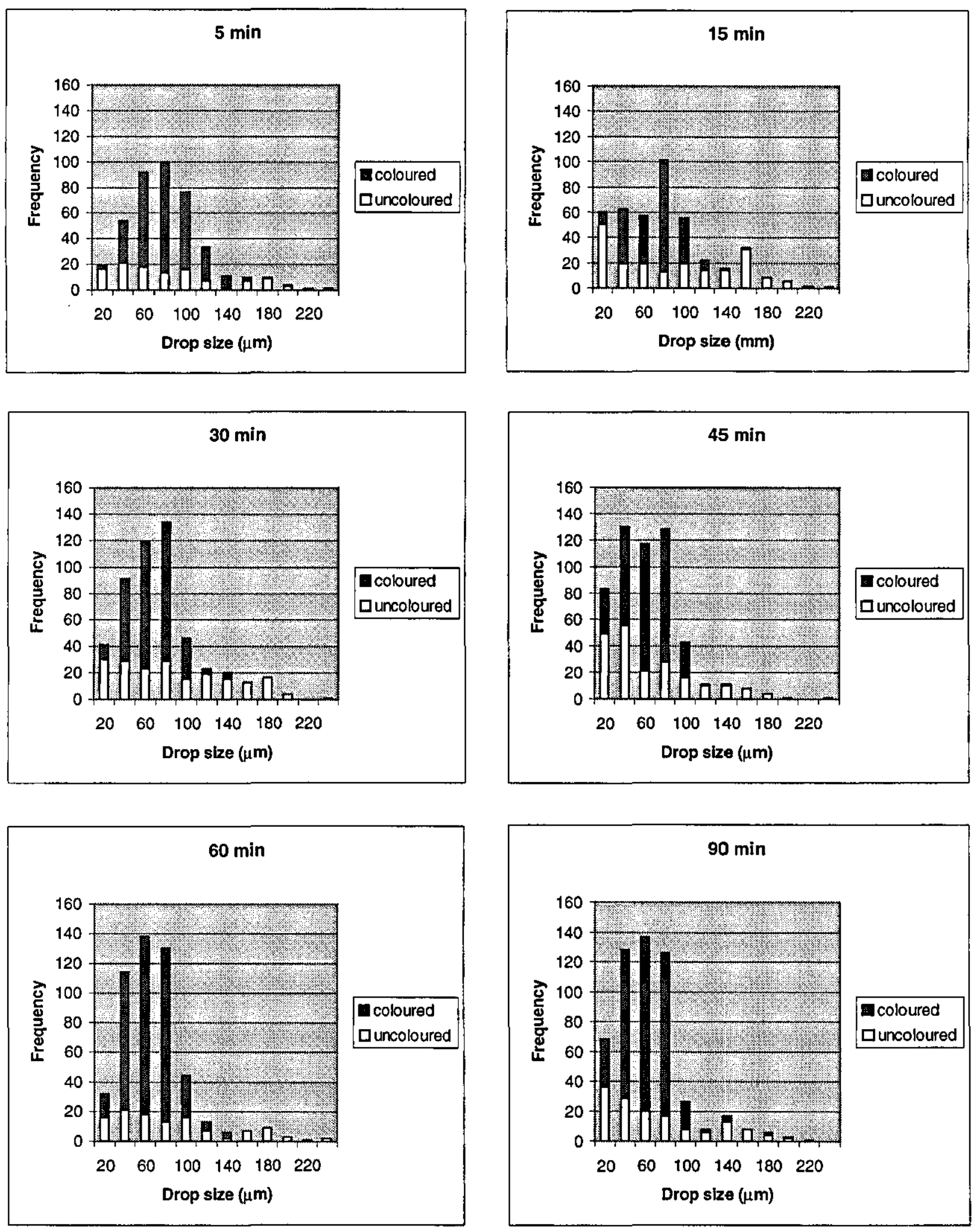

Figure 4.15: Drop size distribution for batch mixing of styrene with 15 wt $\%$ PS dispersed phase at $350 \mathrm{rpm}$ (continue on next page) 

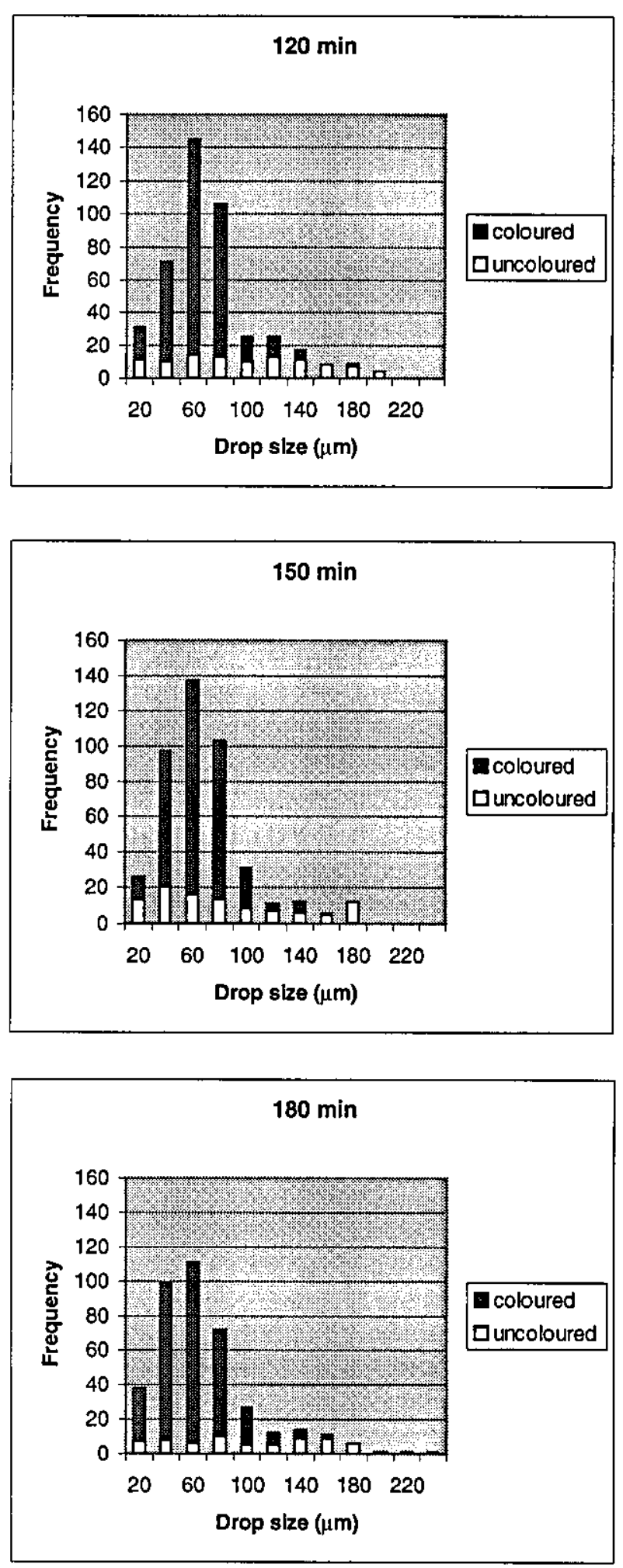

Figure 4.15 (continued) 


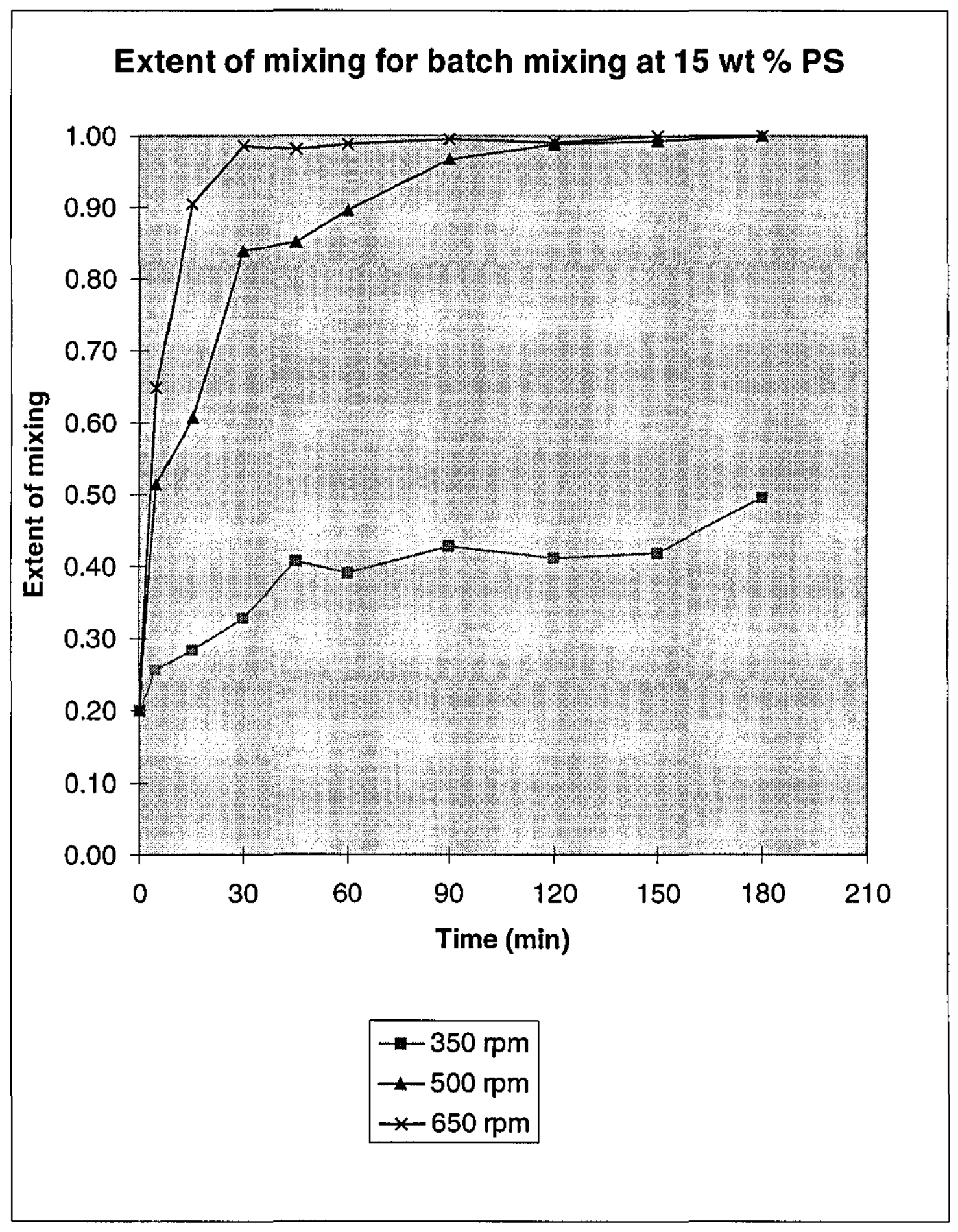

Figure 4.16: Extent of mixing for $15 \mathrm{wt} \%$ PS dispersed phase running at different stirrer speeds 

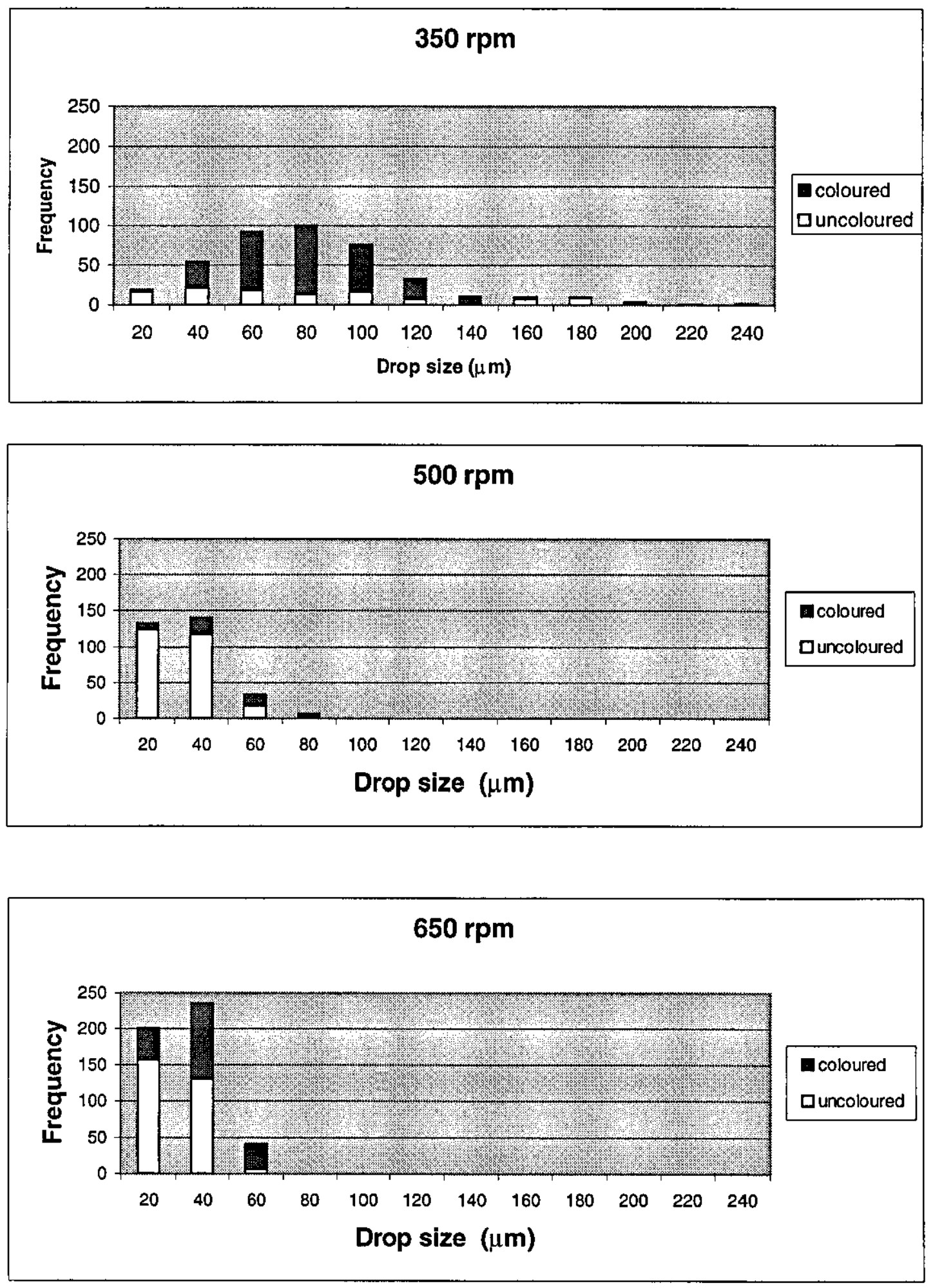

Figure 4.17: Drop size distribution at 5 minutes for $15 \mathrm{wt} \%$ polystyrene solution dispersed phase at different stirring speeds 
As explained in the previous section, drop size plays an important role in determining the rate of mixing between drops. Therefore the stirring speed can be said to affect the drop size of the dispersion then that affects the drop mixing rate between the drops. These results show that an increase in the turbulence intensity increases the breakage rate as well as the coalescence rate.

Figure 4.18 shows the drop size distribution after the mixing at 180 minutes for $15 \mathrm{wt}$ $\%$ polystyrene solution dispersed phase at different stirring speed. The results show that the drop size distribution at $500 \mathrm{rpm}$ and $650 \mathrm{rpm}$ have nearly similar shapes with all drops being coloured. While for the drop mixing at $350 \mathrm{rpm}$, all drops are not coloured and it can be seen that uncoloured drops exist in the whole range of drop sizes. At this stirring speed also, the drop size distribution at 180 minutes is still as wide as at 5 minutes.

\subsubsection{Effect of concentration of PVA on batch mixing}

The parameter to be studied in this subsection is the effect of suspending agent concentration on the mixing rate between coloured and uncoloured drops. For this purpose, a higher concentration of $0.05 \mathrm{wt} \%$ PVA was used. The mixing experiment was run at $500 \mathrm{rpm}$ and the other experimental procedure and conditions were the same as described previously. Since using a higher concentration of PVA resulted in very low drop size in the dispersed phase of $5 \mathrm{wt} \%$ and $10 \mathrm{wt} \%$ of polystyrene solution, a very poor image of coloured and uncoloured drops was produced. Therefore only mixing experiments of $15 \mathrm{wt} \%$ and $20 \mathrm{wt} \%$ polystyrene solution were performed.

Figure 4.19 shows the extent of mixing for the batch mixing using $0.05 \mathrm{wt} \%$ of PVA at $500 \mathrm{rpm}$. The results indicate that a higher viscosity of dispersed phase has a higher coalescence rate between coloured and uncoloured drops. These results are similar to batch mixing experiments that were run at $500 \mathrm{rpm}$ using $0.02 \mathrm{wt} \%$ PVA. At this experimental condition, using $0.05 \mathrm{wt} \%$ PVA, drop size plays an important role in determining the drop mixing rate as explained previously. 

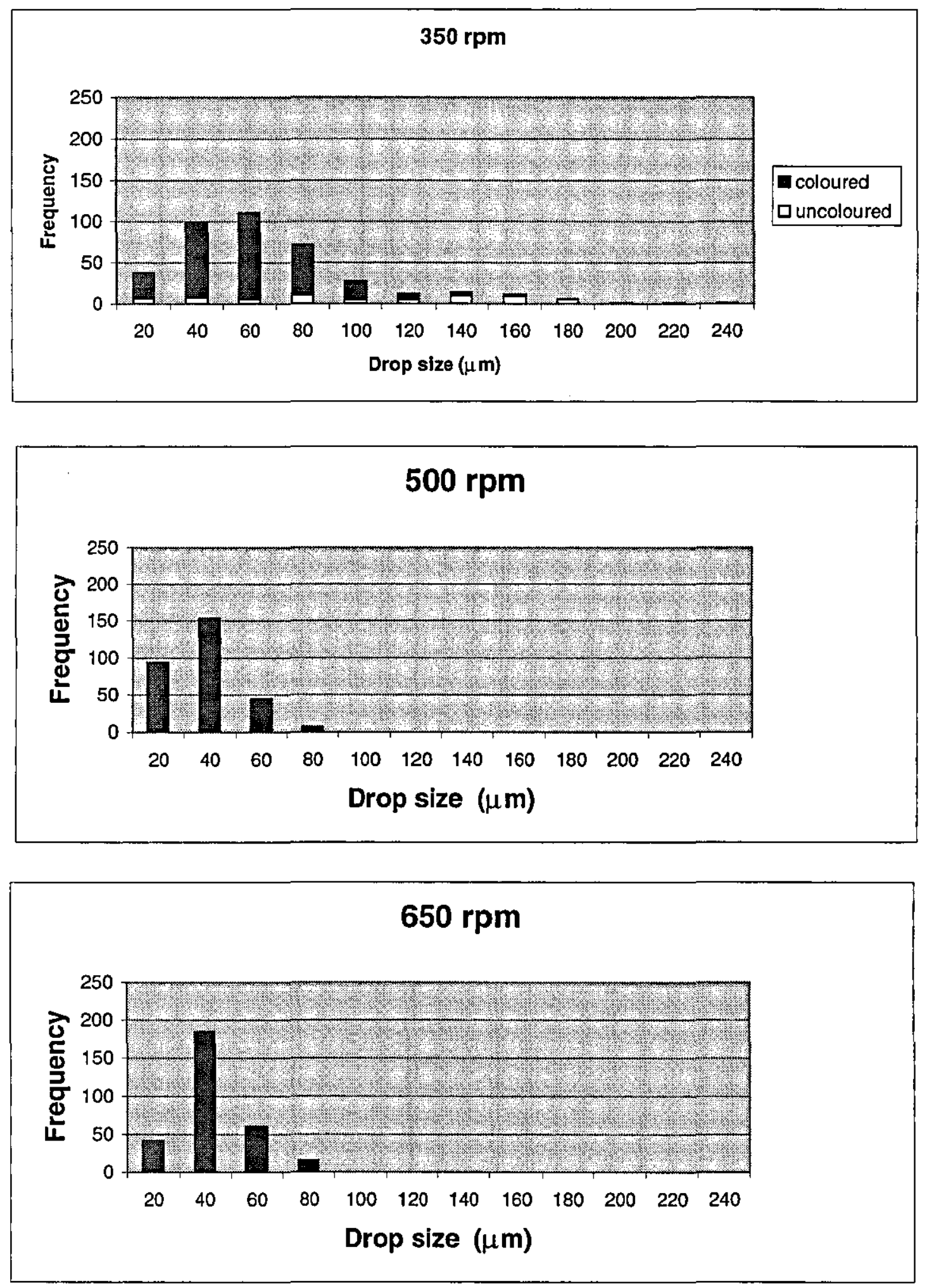

Figure 4.18: Drop size distribution at 180 minutes for $15 \mathrm{wt} \%$ polystyrene solution dispersed phase at different stirring speeds 


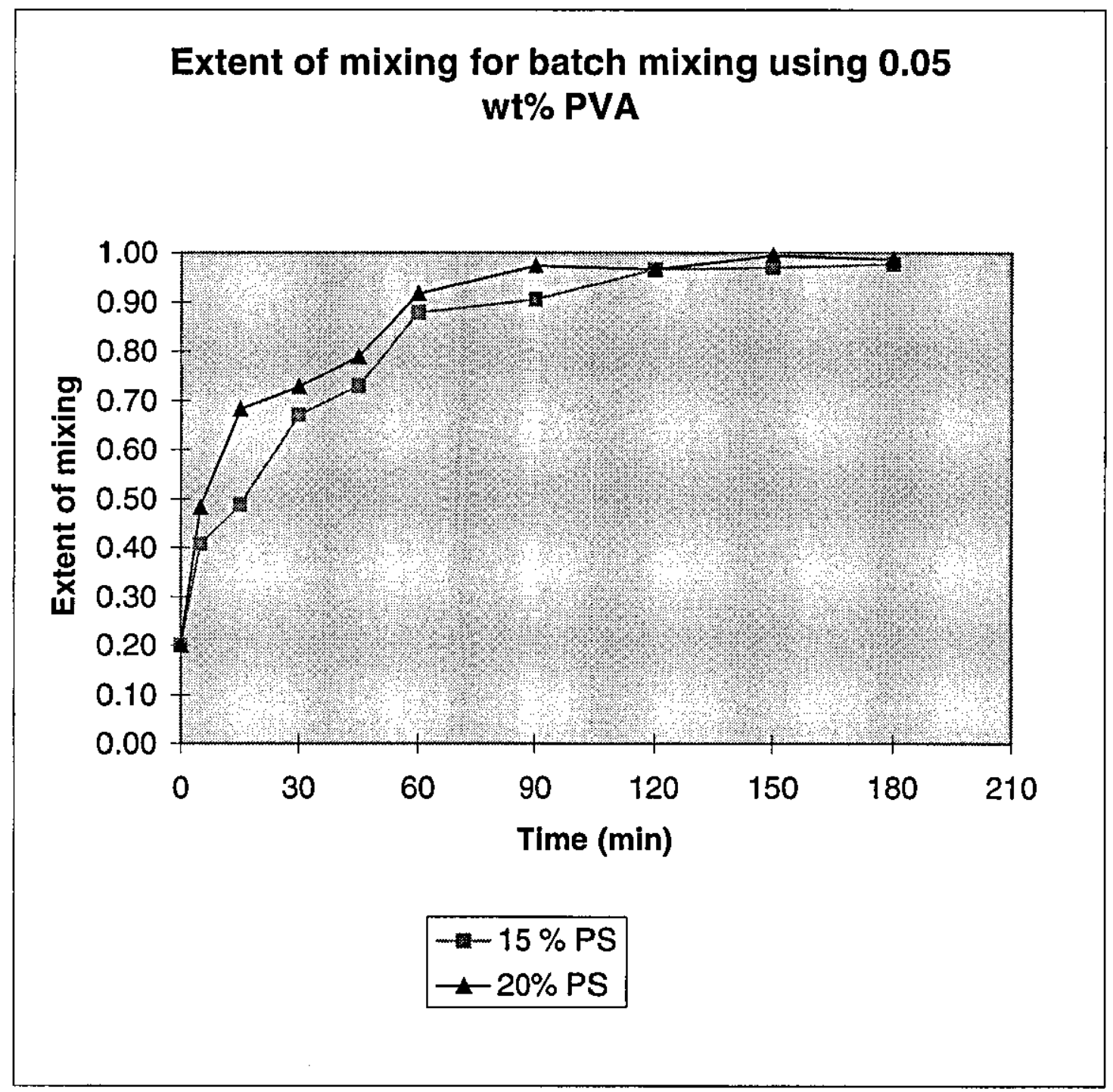

Figure 4.19: Extent of mixing for batch mixing using $0.05 \mathrm{wt} \%$ PVA at $500 \mathrm{rpm}$ 
Figure 4.20 shows the comparison of extent of mixing between the batch mixing using $0.02 \mathrm{wt} \%$ and $0.05 \mathrm{wt} \%$ PVA running at $500 \mathrm{rpm}$. It can be seen that the batch mixing using a higher concentration of PVA has a slower coalescence rate. This is due the fact that the quantities of PVA available in the higher concentration of PVA cover and protect the uncoloured drops more efficiently compared to lower concentration of PVA. However this suggestion is based on amount of PVA used.

Figure 4.21 shows the drop size distribution at 180 minutes of batch mixing using $0.02 \mathrm{wt} \%$ and $0.05 \mathrm{wt} \%$, respectively at $500 \mathrm{rpm}$. The drop size for batch mixing using $0.05 \mathrm{wt} \%$ PVA is smaller and has a wider drop size distribution than that obtained at a concentration of $0.02 \mathrm{wt} \%$ PVA. These results confirm that the amount of suspending agents plays an important role in controlling the coalescence rate as well as the droplet size and size distribution.

\subsubsection{Effect of type of suspending agent on batch drop mixing}

In order to study the effect of type of suspending agent on the batch mixing of the dispersion, the experiments were run using $0.02 \mathrm{wt} \%$ Tween 20 at $500 \mathrm{rpm}$. Other experimental procedures and conditions remained the same as described previously. Figure 4.22 shows the extent of mixing using $0.02 \mathrm{wt} \%$ Tween 20 for 5 wt $\%, 10 \mathrm{wt} \%$ and $15 \mathrm{wt} \%$ polystyrene solution dispersed phase, respectively. The results indicate that as the viscosity of the dispersed phase increased, the rate of mixing between coloured and uncoloured drops increased. These results again are similar to those in the experiments using $0.02 \mathrm{wt} \%$ PVA running at $500 \mathrm{rpm}$.

In order to compare the effect of suspending agent on the drop mixing, the extent of mixing of 0.02 wt \% PVA and 0.02 wt $\%$ Tween 20 for 15 wt \% PS solution dispersed phase were plotted in figure 4.23. The results show that mixing rate in the dispersion using $0.02 \mathrm{wt} \%$ Tween 20 is slower than that using $0.02 \mathrm{wt} \%$ PVA. Therefore the results indicate that Tween 20 is much more efficient in stabilizing and protecting the uncoloured drops as the coalescence rate is relatively low compared to PVA. However this comparison was done on a weight basis of the surfactants. 


\section{Extent of mixing for batch mixing at $15 \mathrm{wt} \% \mathrm{PS}$}

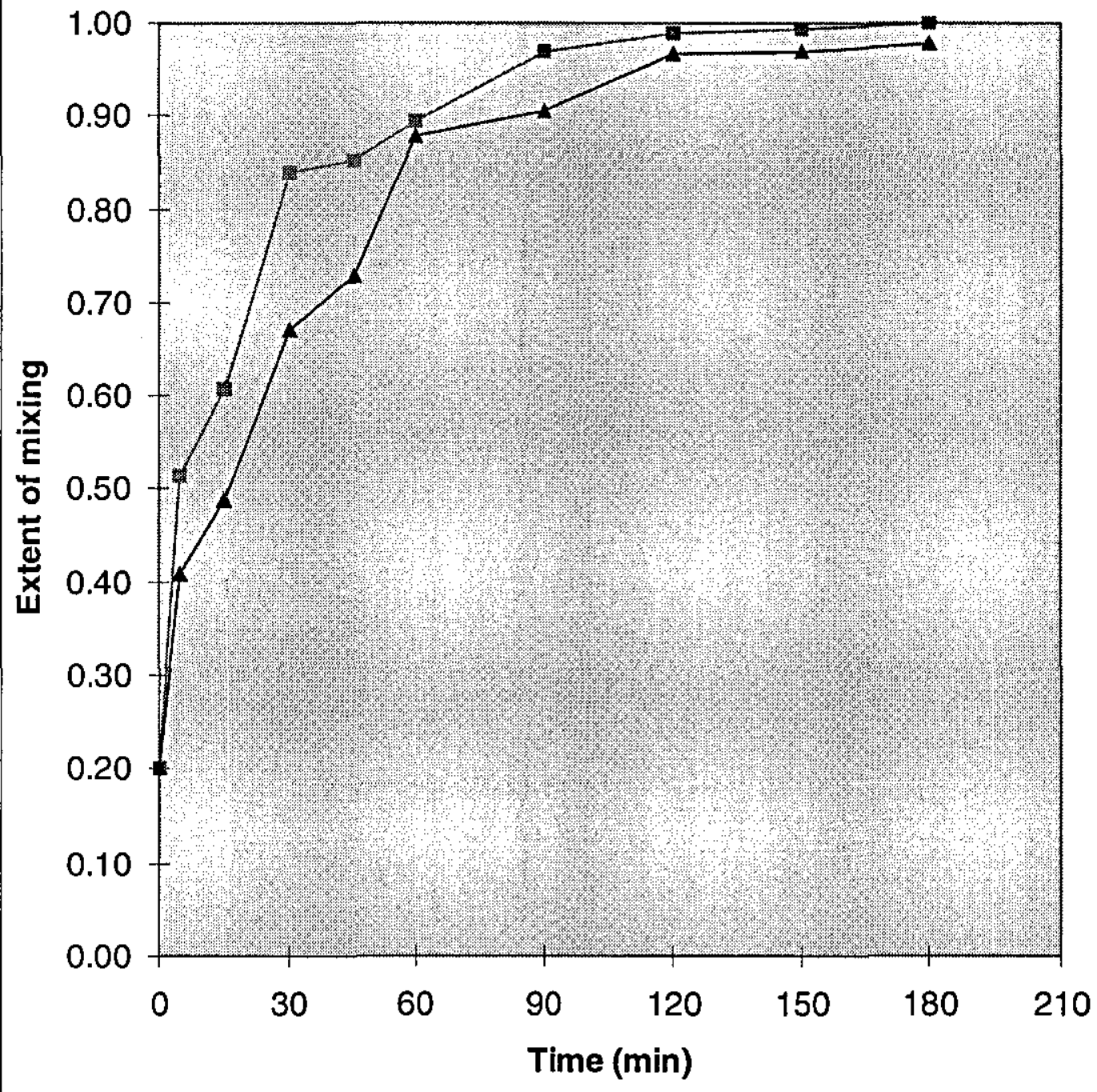

$-0.02 w \%$ PVA
$-0.05 w \%$ PVA

Figure 4.20: Extent of mixing for batch mixing using two different concentration of PVA 

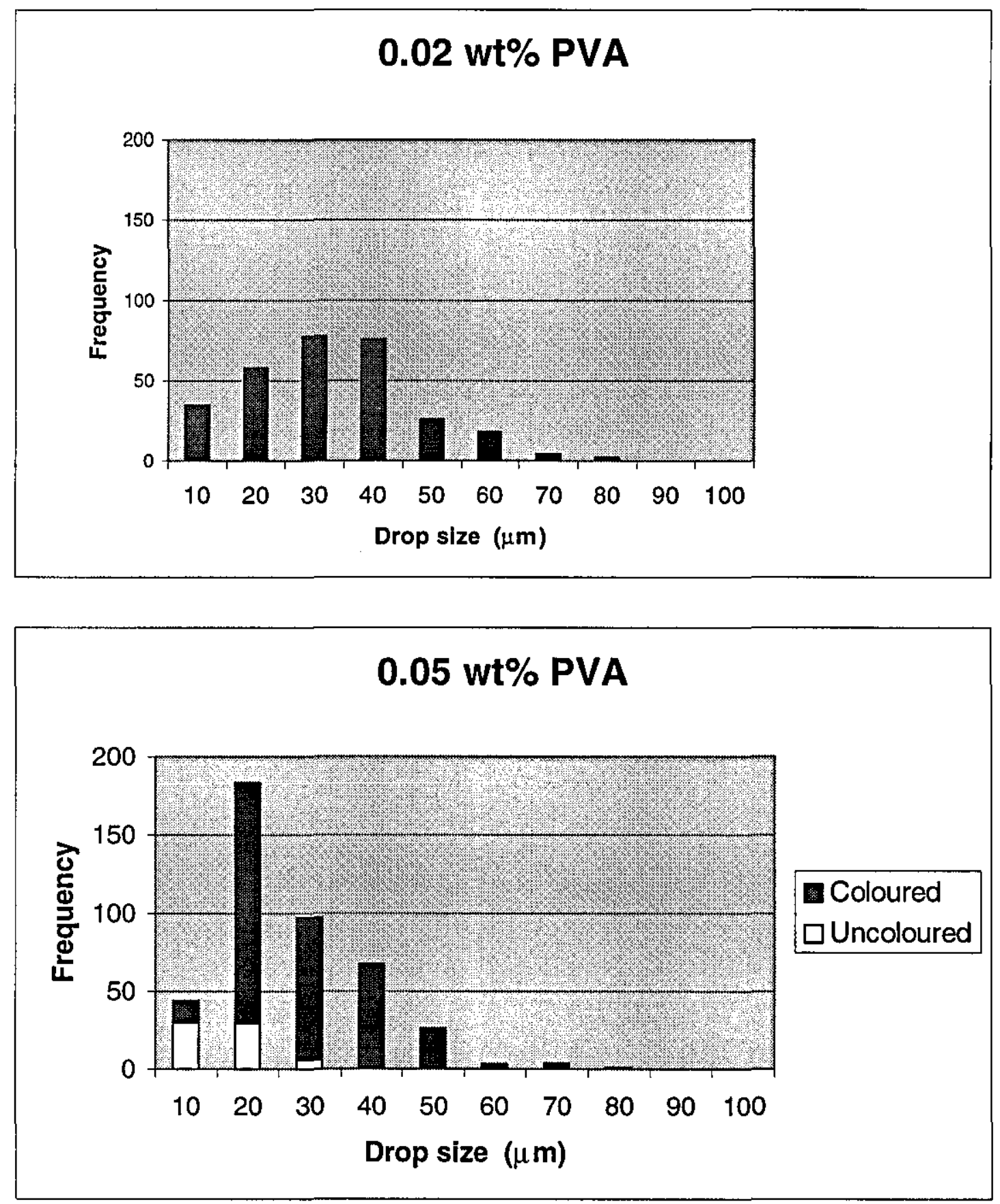

Figure 4.21: Drop size distribution at 180 minutes of batch mixing for two different concentration of PVA 


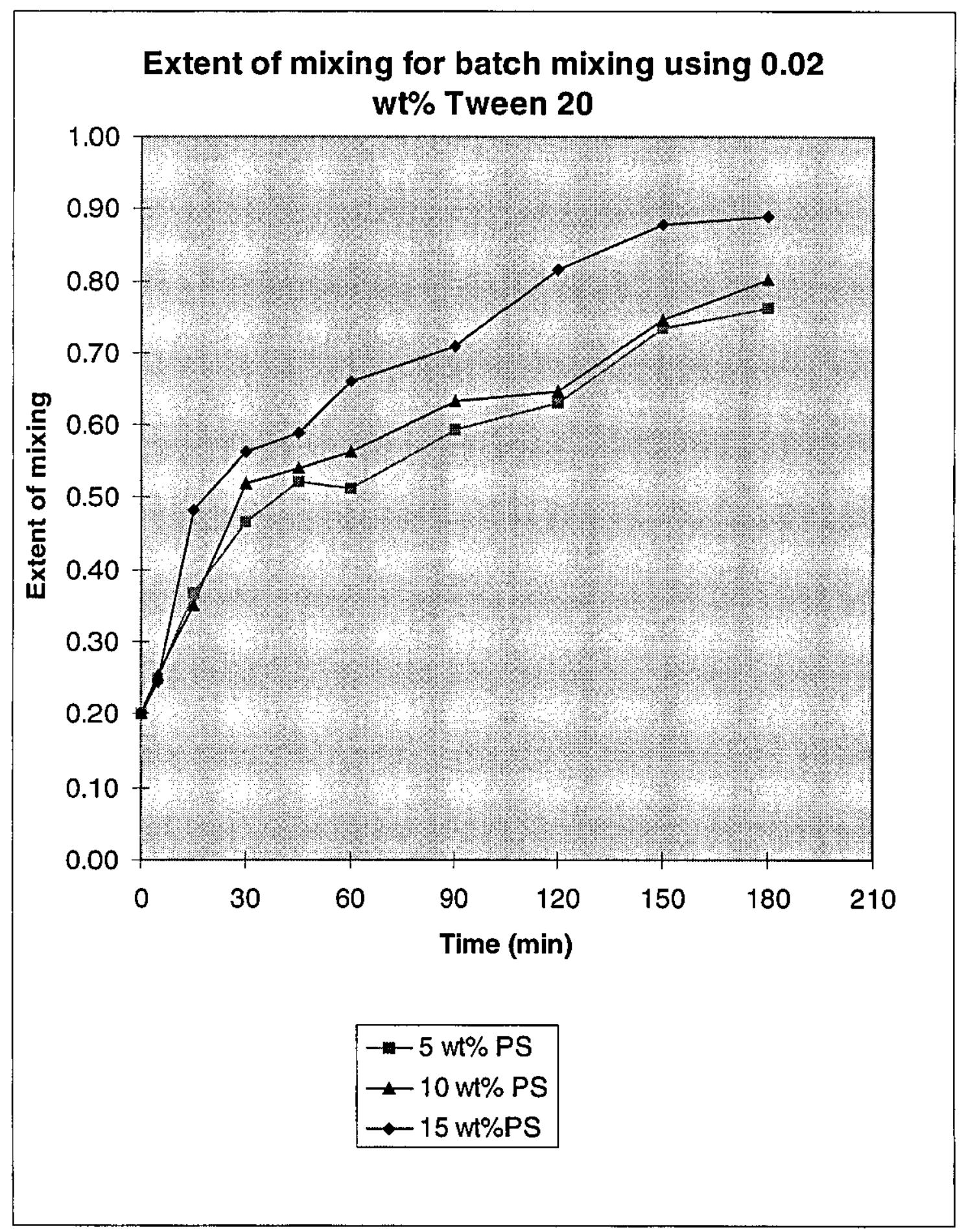

Figure 4.22: Extent of mixing for batch mixing using $0.02 \mathrm{wt} \%$ Tween 20 for different viscosity of dispersed phase. 


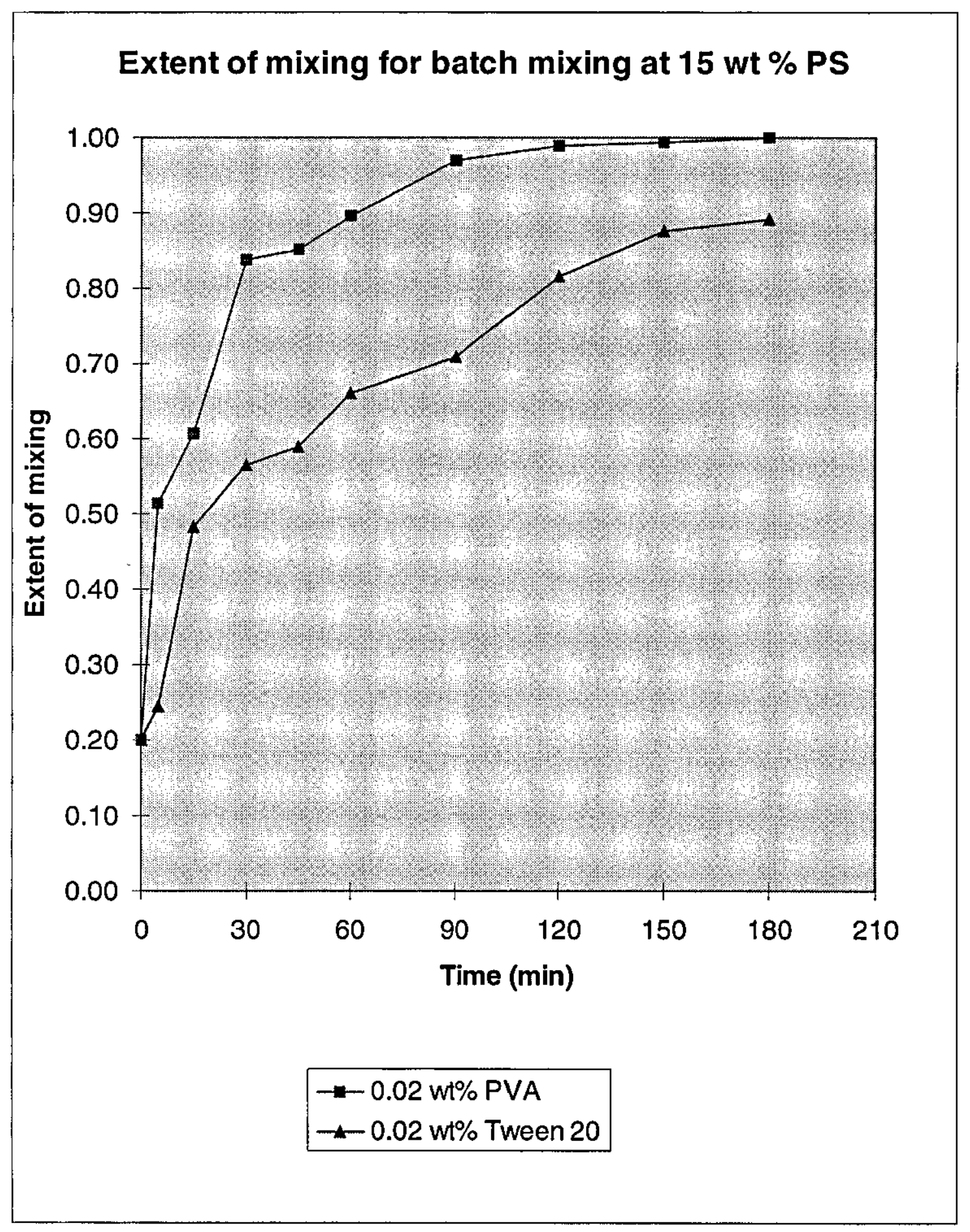

Figure 4.23: Extent of mixing for batch mixing at $15 \mathrm{wt} \%$ PS dispersed phase using different type of surfactant 
The drop size distributions for drop mixing using $0.02 \mathrm{wt} \%$ PVA and $0.02 \mathrm{wt} \%$ Tween 20 at 180 minutes are shown in figure 4.24. The concentration of dispersed phase was at $15 \mathrm{wt} \%$ polystyrene solution. The results indicate that mixing using Tween 20 produced higher drop size and wider drop size distribution. It also shows that for the Tween 20 batch mixing, although the drop sizes are bigger, the mixing also occurs at high drop size as in the batch mixing using $0.02 \mathrm{wt} \%$ PVA at $500 \mathrm{rpm}$ and $650 \mathrm{rpm}$. From this observation, it is noticed that type of suspending agent used also plays an important role in determining the coalescence rate despite the large drop size. 

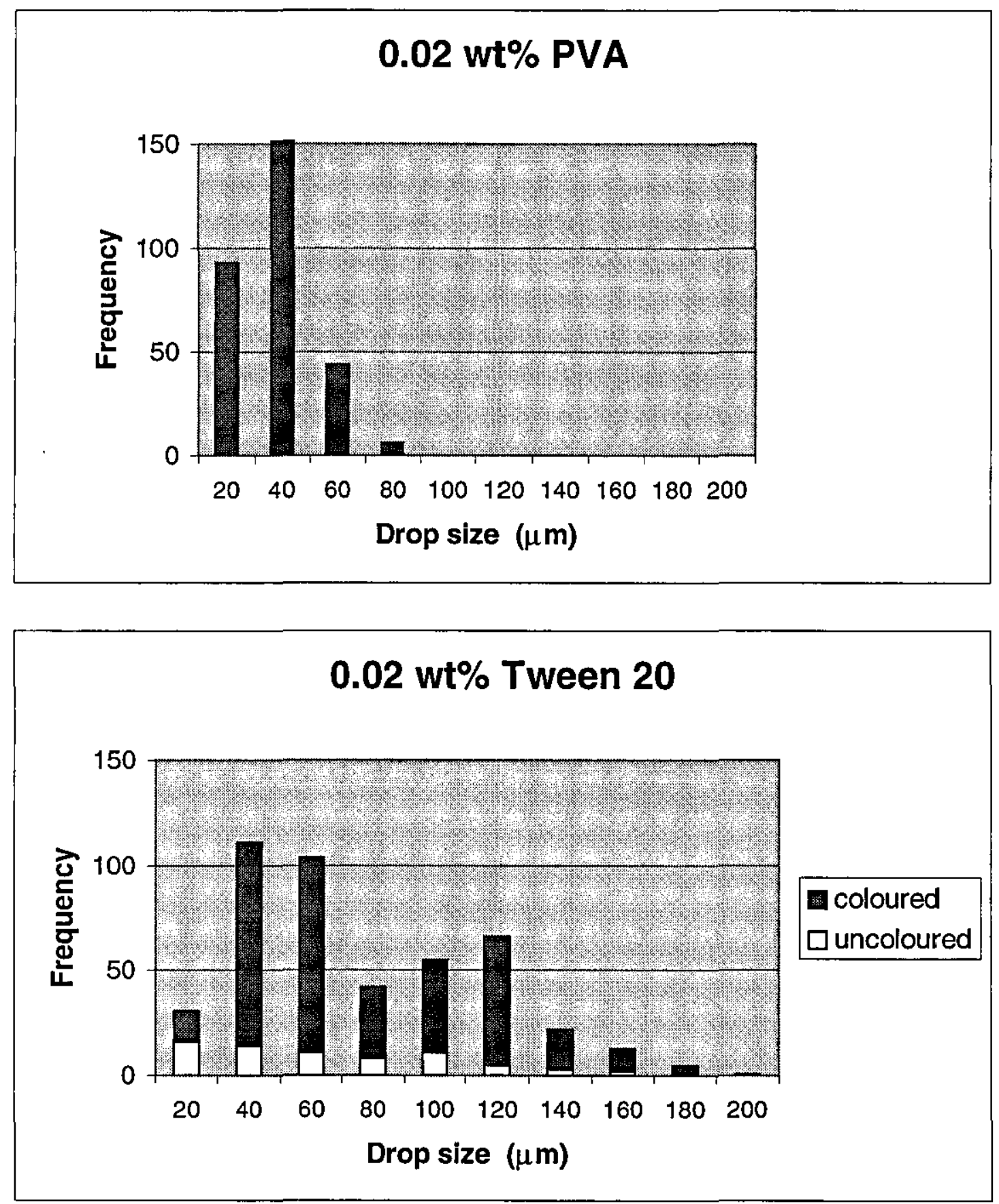

Figure 4.24: Drop size distribution for batch mixing at 180 minutes using different surfactant 


\subsection{Mixing of two stabilised dispersions}

The purpose of the experiments was to study the drop mixing behavior for the mixing between two stabilised dispersions. The major variable studied was the viscosity of the dispersed phase. Two types of experiments were done in this study. First was the mixing of two stabilised dispersions of same viscosities of the dispersed phase. Second was the mixing of two stabilised dispersions with different viscosities of the dispersed phase. These experiments have an advantage of knowing the drop size and drop size distribution for both dispersions before the mixing was performed. Experiments of batch mixing with the same condition as the dispersed phase mixing were also run in order to compare their behavior of mixing.

Before the mixing of the two dispersions was done, premixing dispersion was performed in each vessel containing $50 \mathrm{ml}$ dispersed phase and $450 \mathrm{ml}$ continuous phase. One of the dispersed phases contained dye in order to determined the extent of mixing between drops after the mixing started. Following are the experimental condition used in these experiments:

$\begin{array}{lll}\text { Temperature } & : & 30^{\circ} \mathrm{C} \\ \text { Stirring speed } & : & 350 \mathrm{rpm} \\ \text { Suspending agent } & : & 0.02 \mathrm{wt} \% \text { PVA }\end{array}$

After two hours of premixing, for the first vessel that containing dye, half of the dispersion volume was drained out. Then, half of the uncoloured dispersion volume in the second vessel was transferred to the first vessel and the mixing started. This procedure was done in order to maintain the same volume condition for the dispersion before and after the mixing of the two stabilised dispersions. The mixing experiments were run for 180 minutes and at each stirring time of $5,15,30,45,60$, 90,120 and 180 minutes, the images of the drops were taken and analysed.

\subsubsection{Mixing of same viscosities of dispersed phase}

In this section, four sets of mixing were run with different viscosity of dispersed phase. The purpose was to study the effect of the dispersed phase viscosity 
on the drop mixing. Following are the four sets of the experiments with different viscosities of dispersed phase:

1) Mixing of $0 \mathrm{wt} \%$ polystyrene solution (coloured) and $0 \mathrm{wt} \%$ polystyrene solution (uncoloured)

2) Mixing of $5 \mathrm{wt} \%$ polystyrene solution (coloured) and $5 \mathrm{wt} \%$ polystyrene solution (uncoloured)

3) Mixing of $10 \mathrm{wt} \%$ polystyrene solution (coloured) and $10 \mathrm{wt} \%$ polystyrene solution (uncoloured)

4) Mixing of $15 \mathrm{wt} \%$ polystyrene solution (coloured) and $15 \mathrm{wt} \%$ polystyrene solution (uncoloured)

The first important observation needed was the behaviour of the dispersion before the mixing of the two dispersions occurred. The images were taken for the premixing dispersion at 180 minutes for both coloured and uncoloured dispersion. These experiments were to determine whether both premixed dispersion have the same behaviour.

Figure 4.25a show the drop size distribution for coloured and uncoloured drops for the premixing experiments at 180 minutes for $0 \mathrm{wt} \%$ polystyrene solution of dispersed phase. They show that the drop size distributions are quite similar. This behaviour indicate that the small amount of the dye added to the dispersed phase doesn't have much affect on the dispersion behaviour. Figure $4.25 \mathrm{~b}$ show the drop size distribution for coloured and uncoloured drops for $10 \mathrm{wt} \%$ polystyrene solution dispersed phase. They also show that both dispersion, coloured and uncoloured have nearly similar behaviour before mixing. However, the $10 \mathrm{wt} \%$ polystyrene solution of dispersed phase has a larger drop size and wider drop size distribution compared to $0 \mathrm{wt} \%$ polystyrene solution of dispersed phase. This phenomena is expected since higher viscosity has contributed larger drop size and wider drop size distribution. These premixing experiments observation confirm that both stabilised dispersions have similar behaviour before the mixing of the two stabilised dispersions happened. 

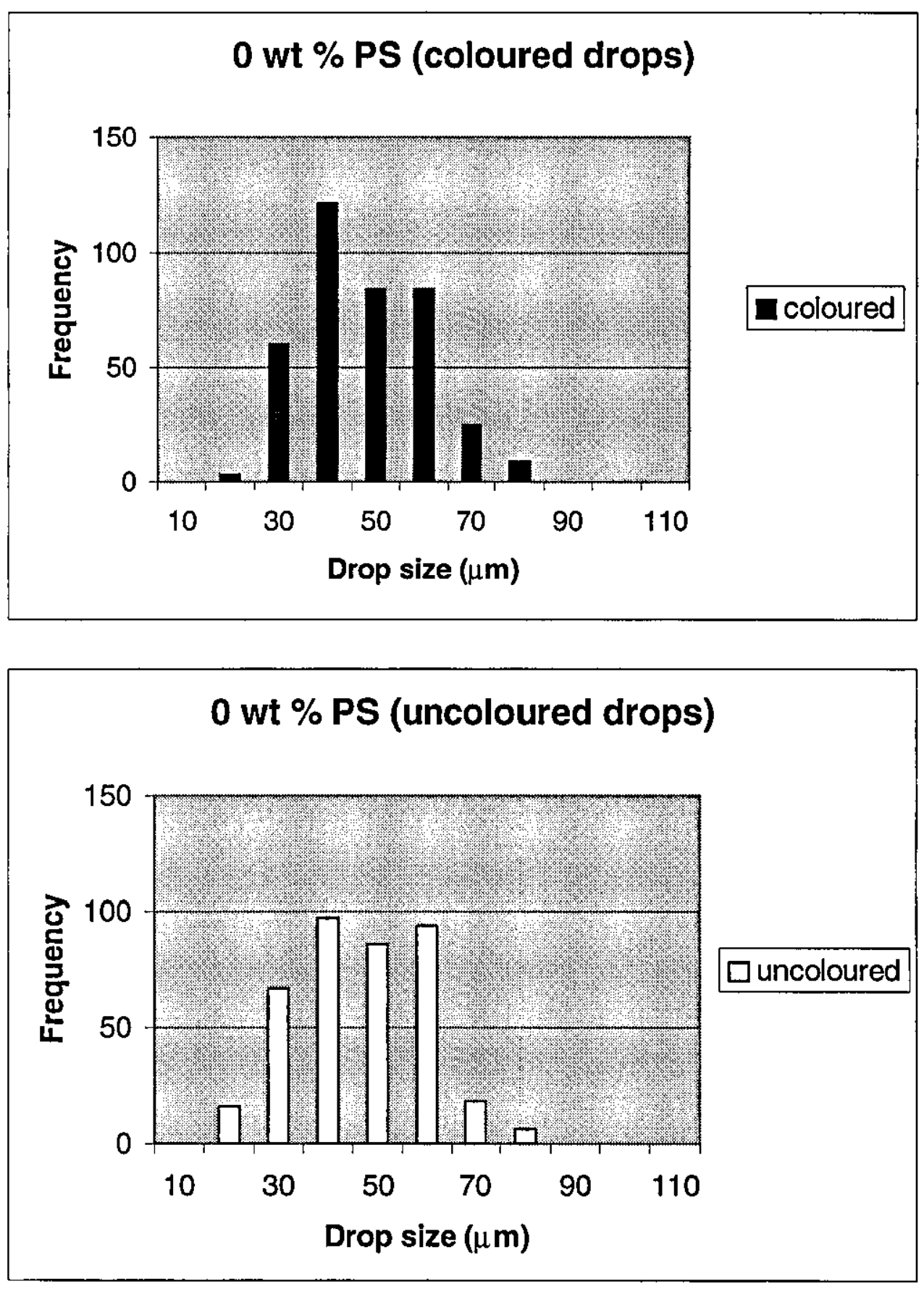

Figure 4.25a: Drop size distribution for premixing after 120 minutes for 0 wt $\%$ polystyrene solution (styrene) disperse phase 

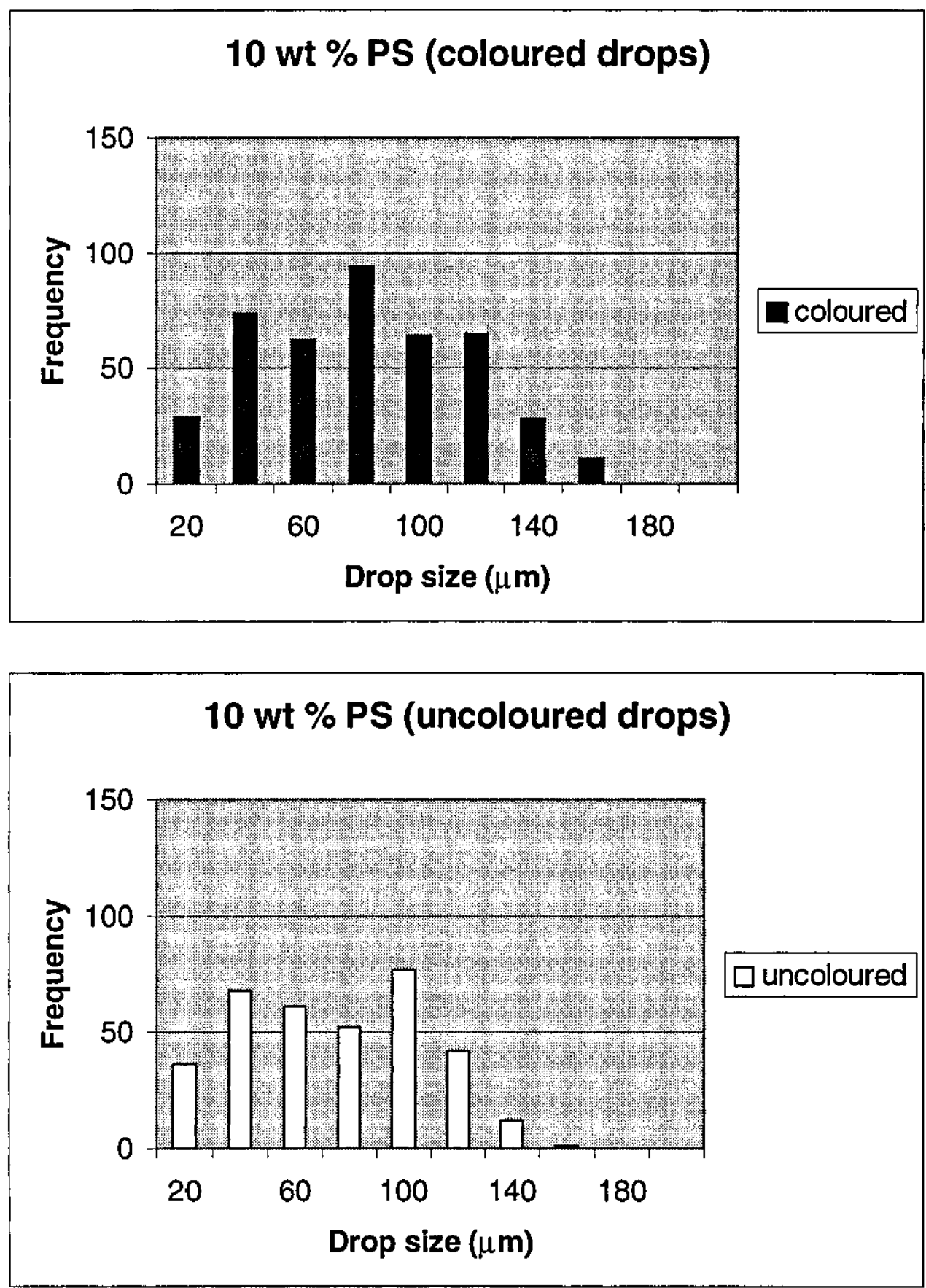

Figure 4.25b: Drop size distribution for premixing after 120 minutes for $10 \mathrm{wt} \%$ polystyrene solution (styrene) disperse phase 
Figure 4.26 shows the extent of mixing of two stabilised dispersions with different viscosity of the dispersed phase. It shows that as the viscosity of the dispersed phase of $0 \mathrm{wt} \%$ PS $+0 \mathrm{wt} \%$ PS increases to $5 \mathrm{wt} \%$ PS $+5 \mathrm{wt} \%$ PS, the mixing rate between coloured and uncoloured drops increases. However as the dispersed phase viscosity increases from $5 \mathrm{wt} \%$ PS $+5 \mathrm{wt} \%$ PS to $10 \mathrm{wt} \%+10 \mathrm{wt} \%$ PS and $15 \mathrm{wt}$ $\%$ PS +15 wt $\%$ PS, the mixing rate decreases. The mixing rate for the highest viscosity in this experiment can be seen take very low compared to the lower viscosity of dispersed phase. As observed in section 4.2 previously, drop size also plays an important role to determine the mixing rate. Therefore analysis of drop size were also needed to be done in order to explained the drop mixing behaviour in these experiments as depicted in figure 4.26.

The comparison between the drop size distribution for the different viscosities of the dispersed phase at 5 minutes after the mixing of the two dispersions is shown in figure 4.27. It can be seen that the mixing of $5 \mathrm{wt} \% \mathrm{PS}+5 \mathrm{wt} \%$ PS dispersed phase gives a wider drop size distribution as well as higher mean drop size compared to the dispersed phase of 0 wt $\%$ PS +0 wt $\%$ PS. The coloured drops at higher drop sizes $(>80 \mu \mathrm{m})$ in the dispersed phase of $5 \mathrm{wt} \%$ PS $+5 \mathrm{wt} \%$ PS contributed higher extent of mixing compared to the dispersed phase of $0 \mathrm{wt} \% \mathrm{PS}+0 \mathrm{wt} \%$ PS. These results show that as the viscosity of the dispersed phase increased, the drop size increases and also the drop size distribution becomes wider. The increase in the drop size has made the coalescence rate between coloured and uncoloured drops increase. As explained in section 4.2, this result is similar to the results produced by Tobin, T. et. al (1990) and Park, J.M. and Blair, L.M. (1975). It can be said that at this experimental condition, from 0 wt $\%$ PS +0 wt $\%$ PS to $5 \mathrm{wt} \%$ PS +5 wt $\%$ PS dispersed phase, the viscosity affects the mixing rate indirectly. Drop size seemed to play an important role in determining the rate of mixing between the drops.

As the dispersed phase increases from $5 \mathrm{wt} \% \mathrm{PS}+5 \mathrm{wt} \%$ PS to $10 \mathrm{wt} \%$ PS $+10 \mathrm{wt}$ $\%$ PS, the rate of mixing reduced, even below the dispersed phase of 0 wt $\%$ PS +0 wt \% PS. The drop size distribution of $10 \mathrm{wt} \%$ PS $+10 \mathrm{wt} \%$ PS reveals that at higher drop size $(>80 \mu \mathrm{m})$, not all drops are coloured. This situation is opposite to the behaviour of the drops at the dispersed phase of $5 \mathrm{wt} \%$ PS $+5 \mathrm{wt} \%$ PS. 


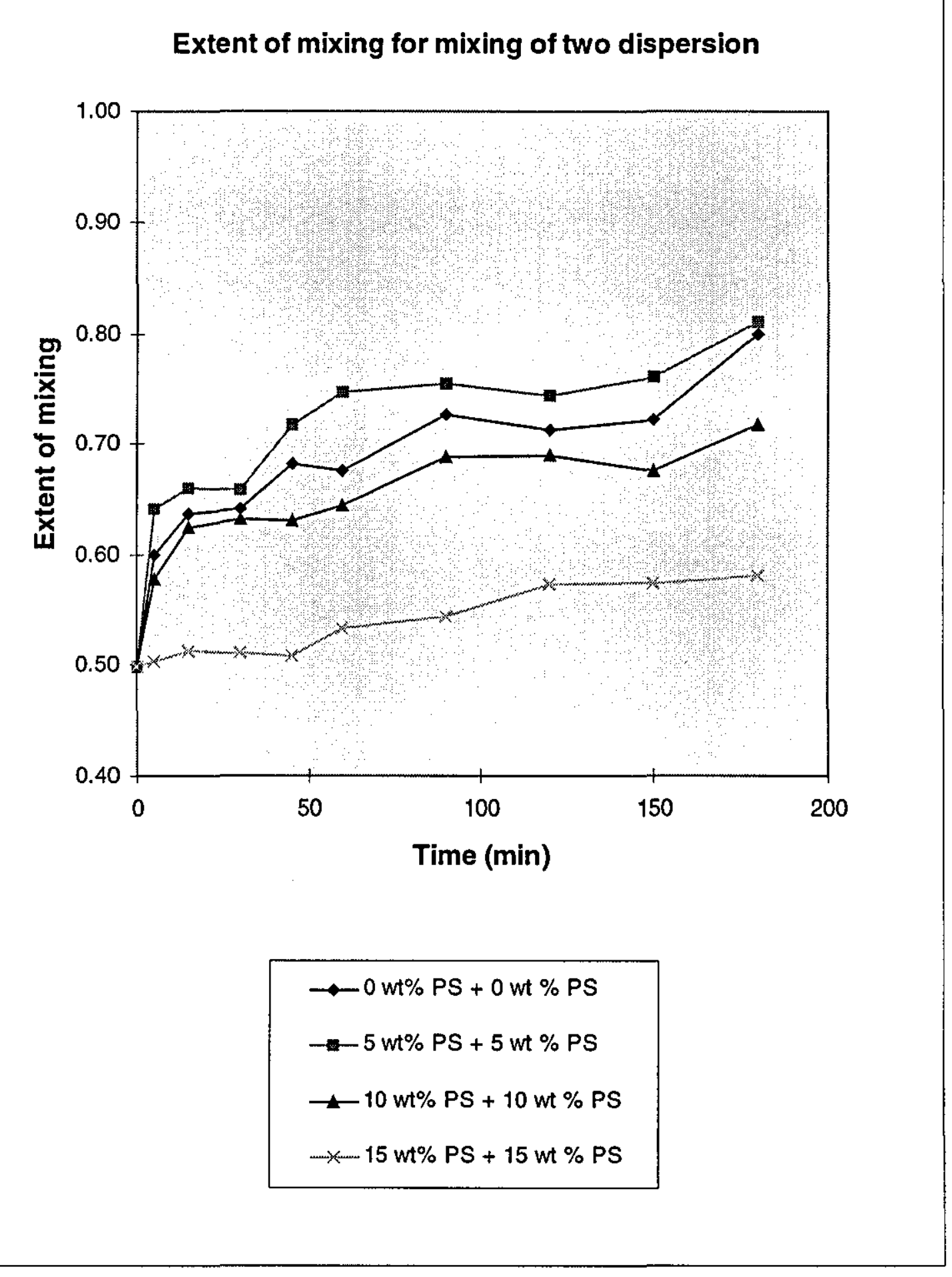

Figure 4.26: Extent of mixing for mixing of two stabilised dispersion with different viscosities of dispersed phase 

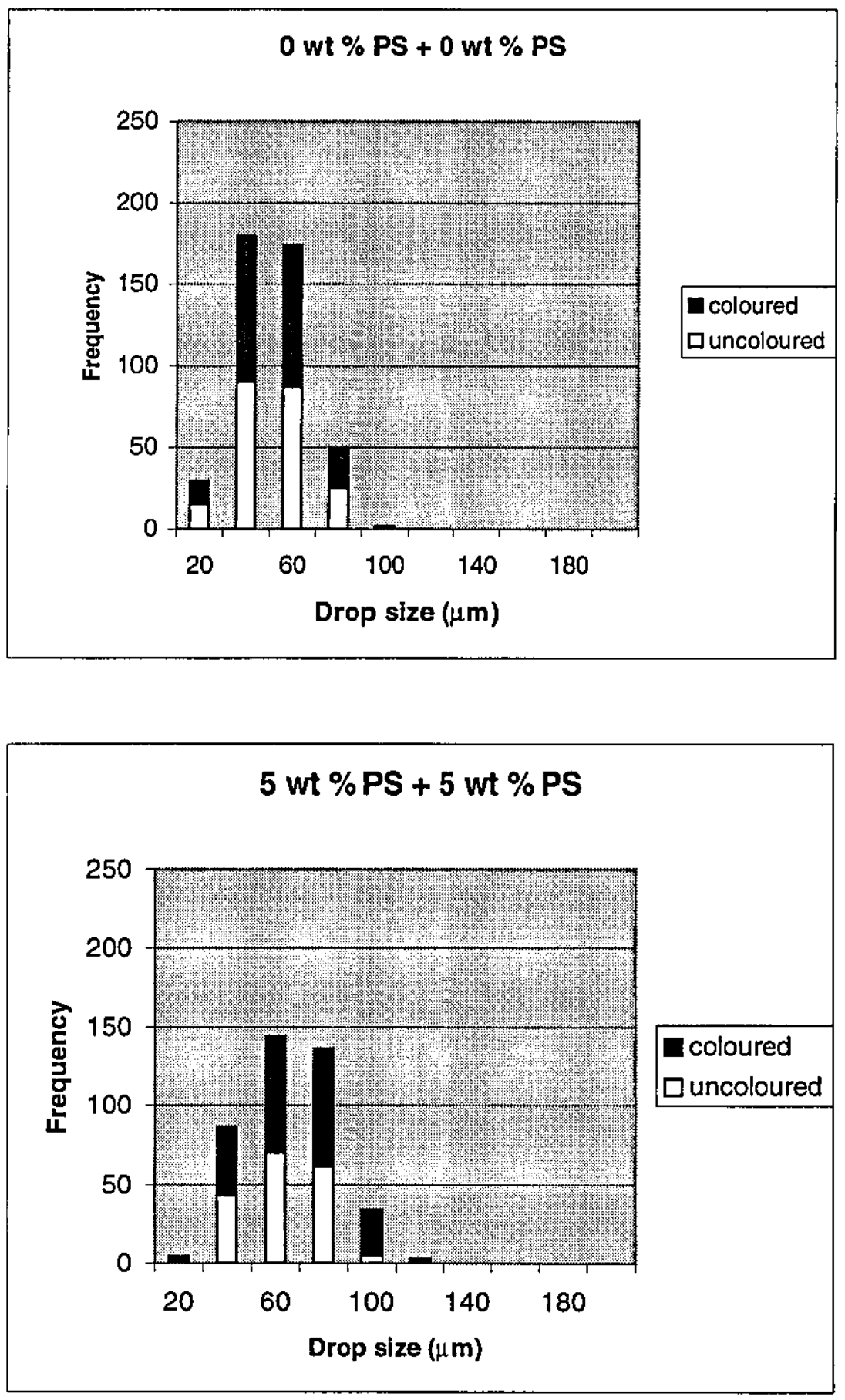

Figure 4.27: Drop size distribution at 5 minutes at different viscosity of dispersed phase for mixing of two dispersion (continue on next page) 

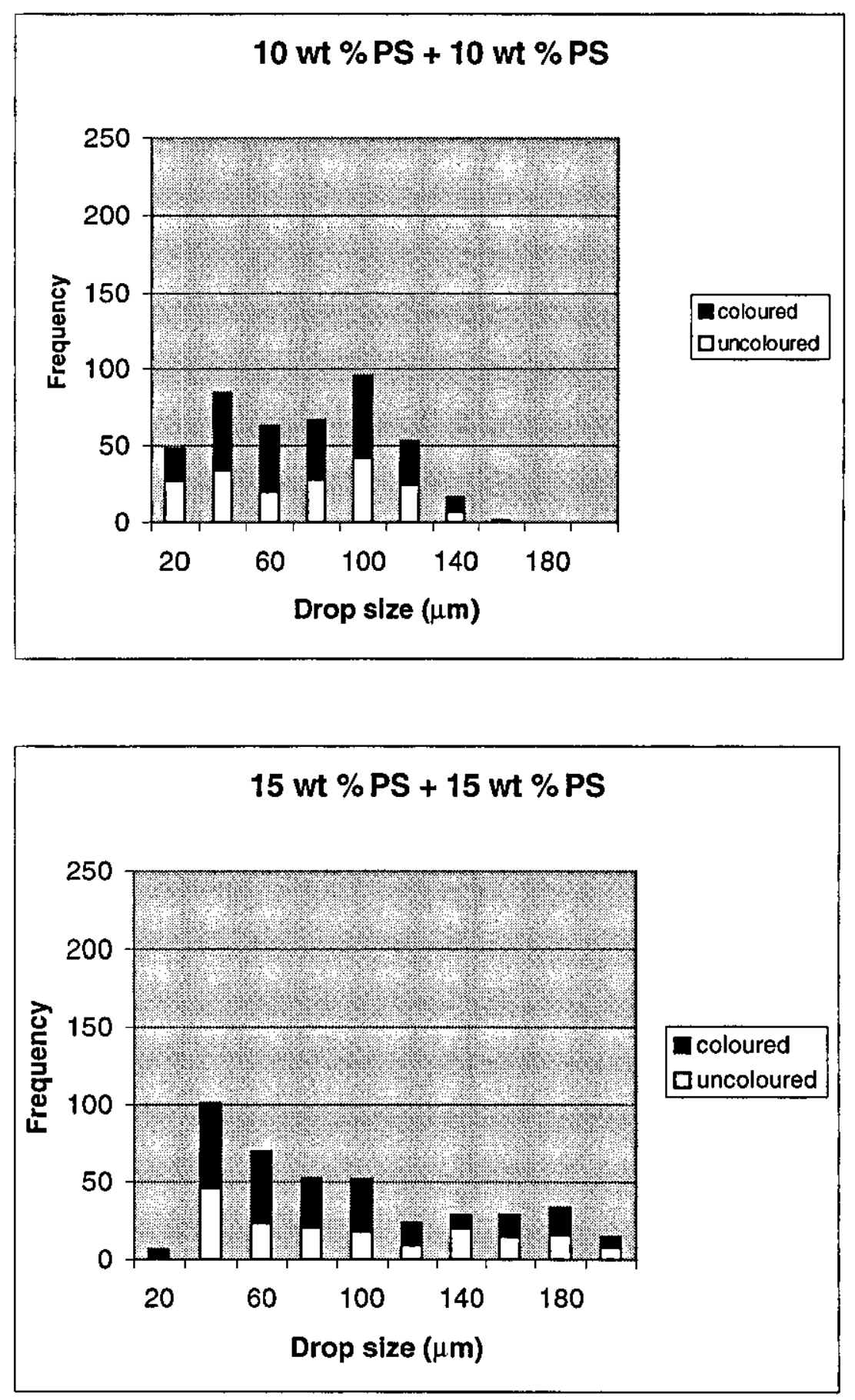

Figure 4.27 (continued) 
This phenomenon has contributed to a lower extent of mixing for the dispersed phase of $10 \mathrm{wt} \% \mathrm{PS}+10 \mathrm{wt} \%$. The result shows that at this stage, viscosity play an important role to determine the rate of mixing between the coloured and uncoloured drops. At 15 wt $\%$ PS +15 wt $\%$ PS dispersed phase, it can be seen clearly that higher drop size doesn't contribute to the higher extent of mixing. These results confirm that at this larger drop size, with increasing drop viscosity, the coalescence efficiency become lower. As explained in batch mixing experiments, an increase in drop phase viscosity will result in a slower drainage rate.

Figure 4.28 shows the comparison between the drop size distribution for the different viscosity of the dispersed phase at 180 minutes after the mixing at the two dispersions. The result also shows the similar behavior as explained for the comparison of the drop size distribution at 5 minutes. These results also confirm that drop size play an important roles in determining the rate of mixing at lower viscosity of drop size. However at higher viscosity of dispersed phase, viscosity play an important role in determining the rate of mixing between drops.

Figure 4.29 shows the transitional behaviour of drop size distribution for the dispersed mixing of $0 \mathrm{wt} \%$ PS $+0 \mathrm{wt} \%$ PS. The results show that the drop size distribution from 5 minutes to 180 minutes after the mixing is quite similar. The increase of total volume of coloured drops indicate that coalescence occur during the mixing process. The similar drop size distribution indicates that the coalescence rate is equal to the breakage rate during the mixing process. In other words, the dispersion is at steady state starting from the start of the mixing of the two dispersions to 180 minutes after the mixing. The steady state behaviour will remain after even 180 minutes. The drop size distribution for the dispersed phase of $0 \mathrm{wt} \%$ PS before the mixing of the two dispersions seemed almost similar to that after mixing. This is because the experiment conditions are the same for before and after the mixing. It also shows that the premixing dispersions are already at steady state. It should be noted that the coalescence and breakage occur on coloured and uncoloured drops. The transitional behavior also shows that the larger drops have higher coalescence efficiency compared to the smaller drops. The larger drops contribute toward the higher extent of mixing. 
The transitional behaviour of drop size distribution for the dispersed mixing of $10 \mathrm{wt}$ $\%$ PS $+10 \mathrm{wt} \%$ PS is shown in figure 4.30. The results also show that their drop size distribution is quite similar from 5 minutes to 180 minutes after the mixing started. These phenomena show that the dispersion is at steady state during the mixing process. It can be conclude that the dispersion will remain at steady state if the premixing dispersions are already at steady state. This is due to the fact that they have the same mixing condition before and after the mixing of the two dispersions. 

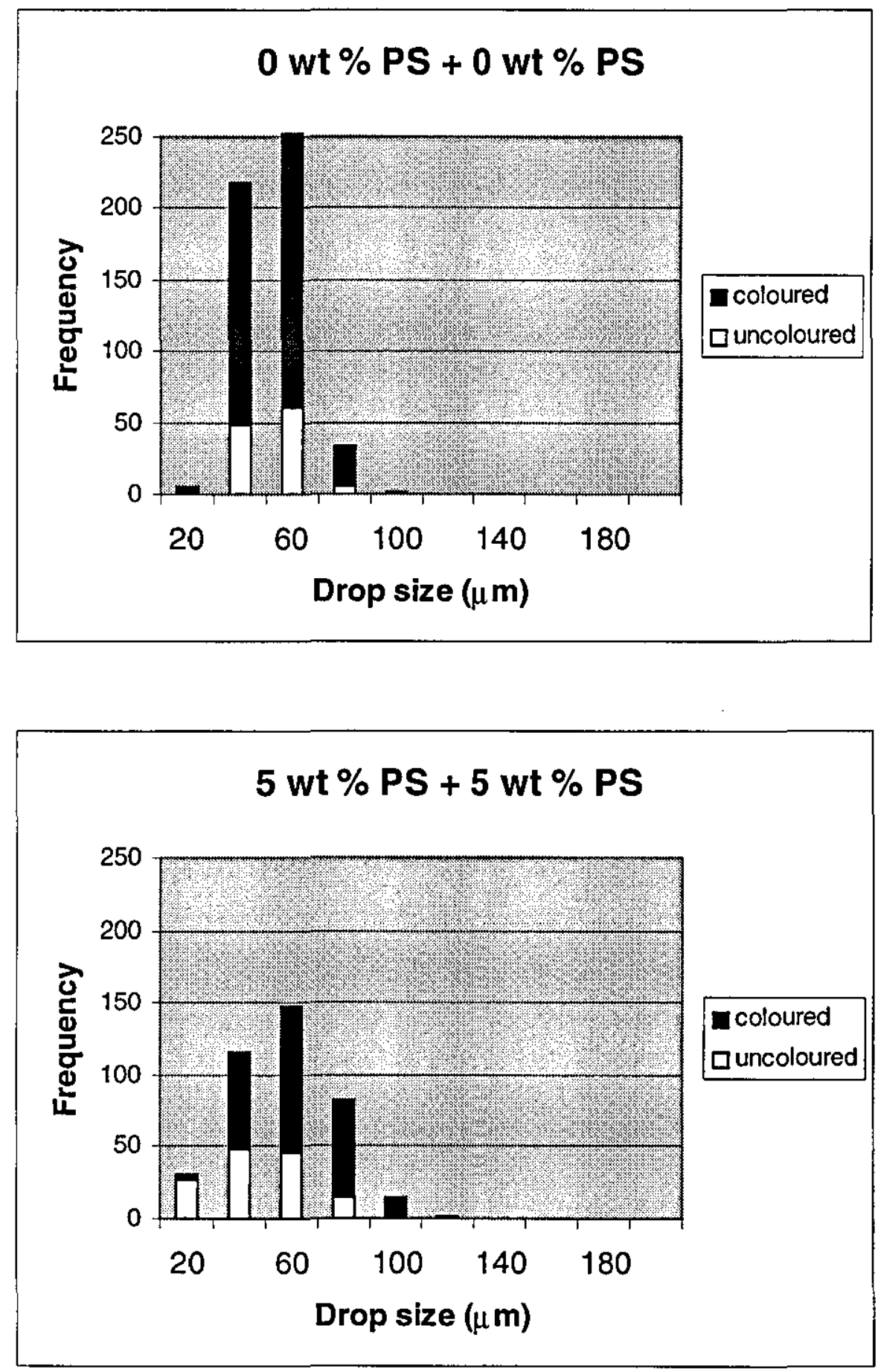

Figure 4.28: Drop size distribution at 180 minutes at different viscosity of dispersed phase for mixing of two dispersions.

(continue on next page) 

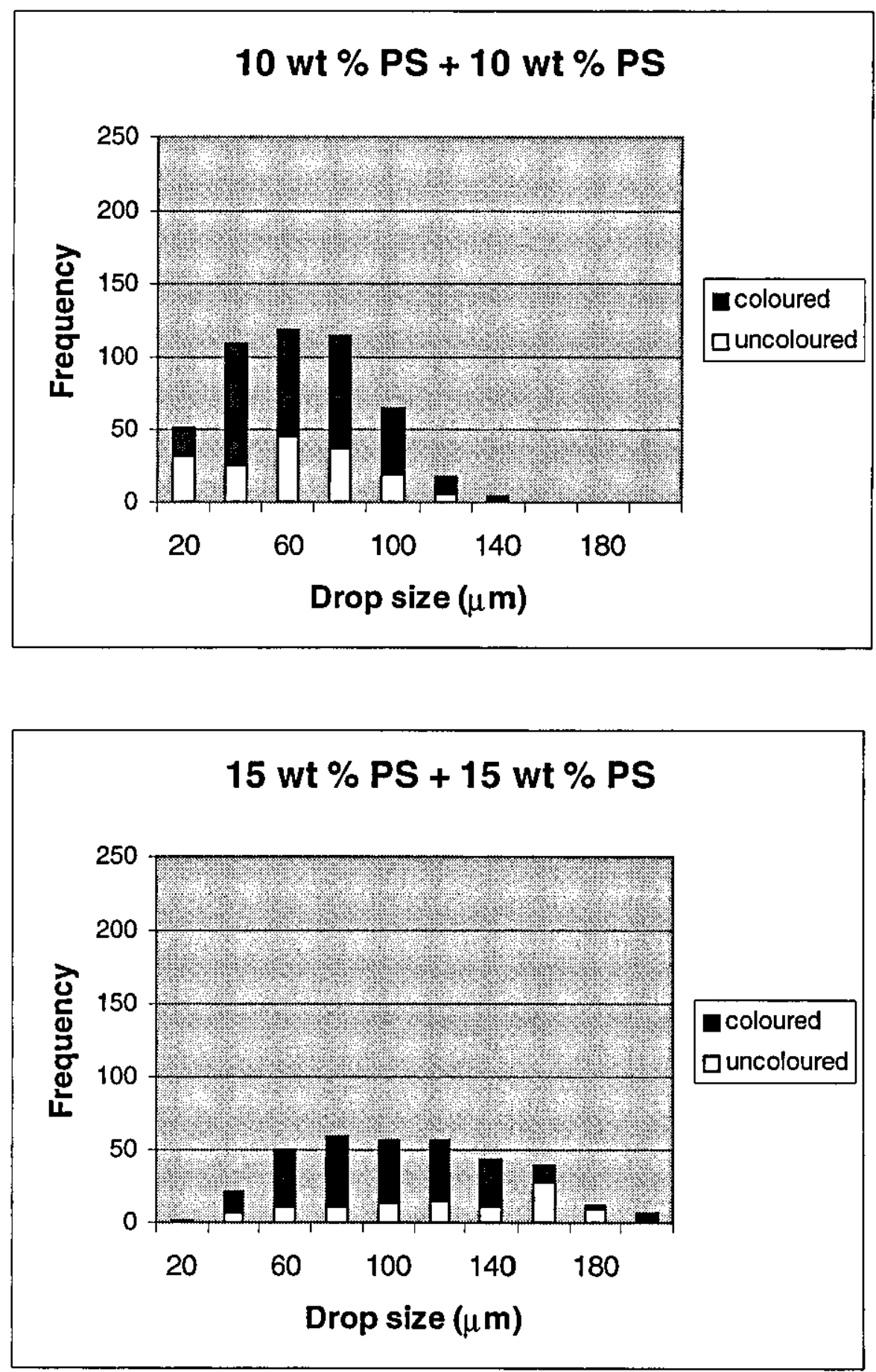

Figure 4.28 (continued) 

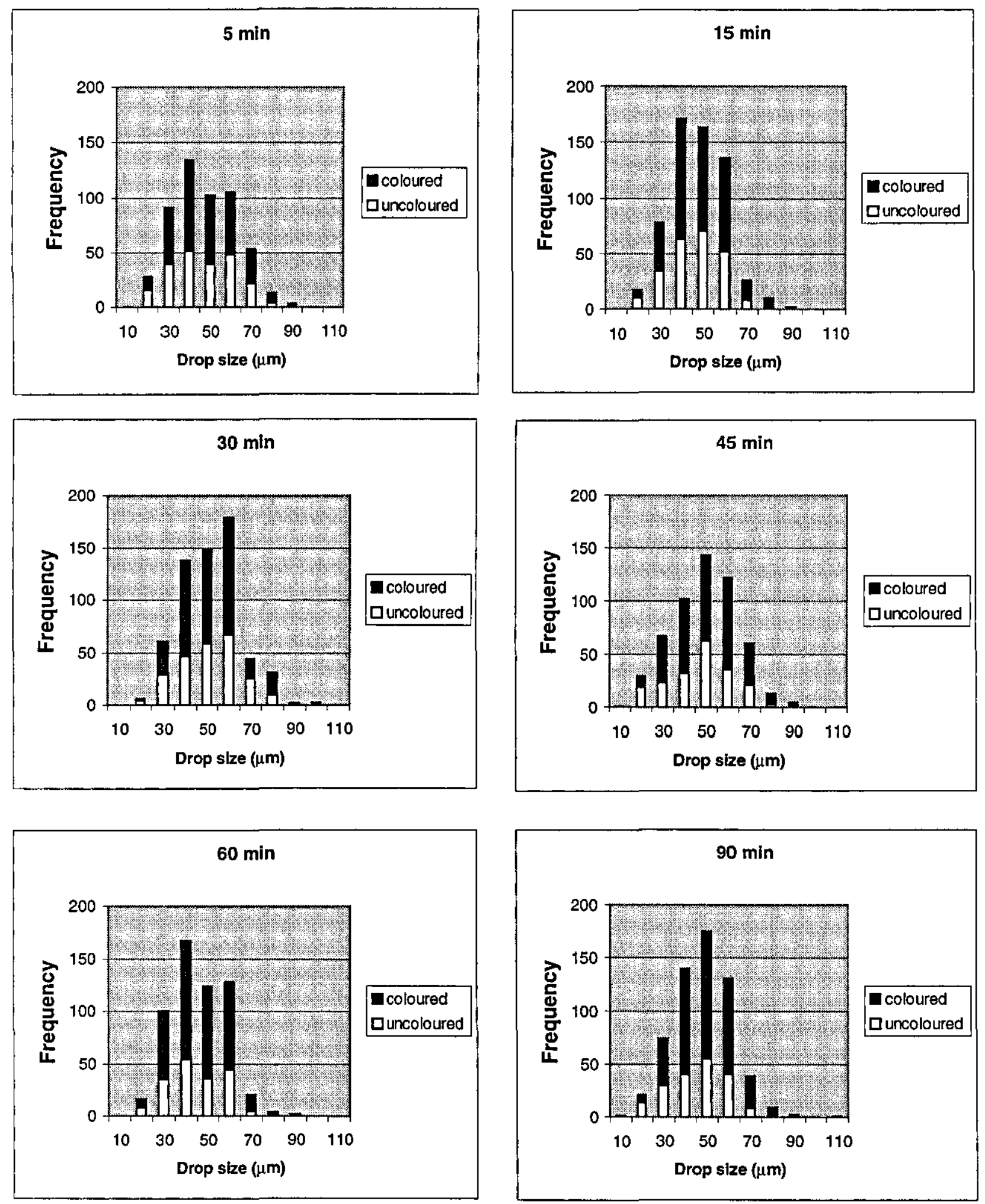

Figure 4.29: Drop size distribution for mixing of two stabilised dispersion of styrene (0 wt \% PS) dispersed phase (continue on next page) 

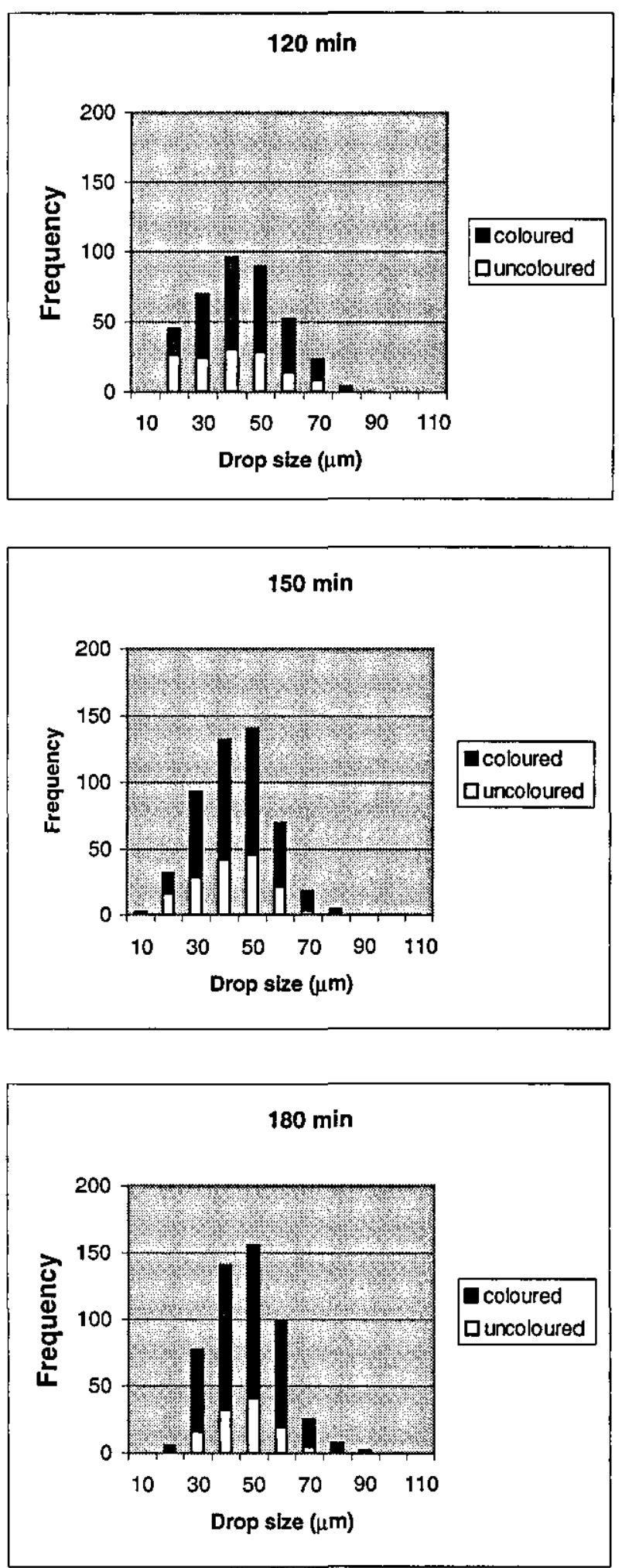

Figure 4.29 (continued) 

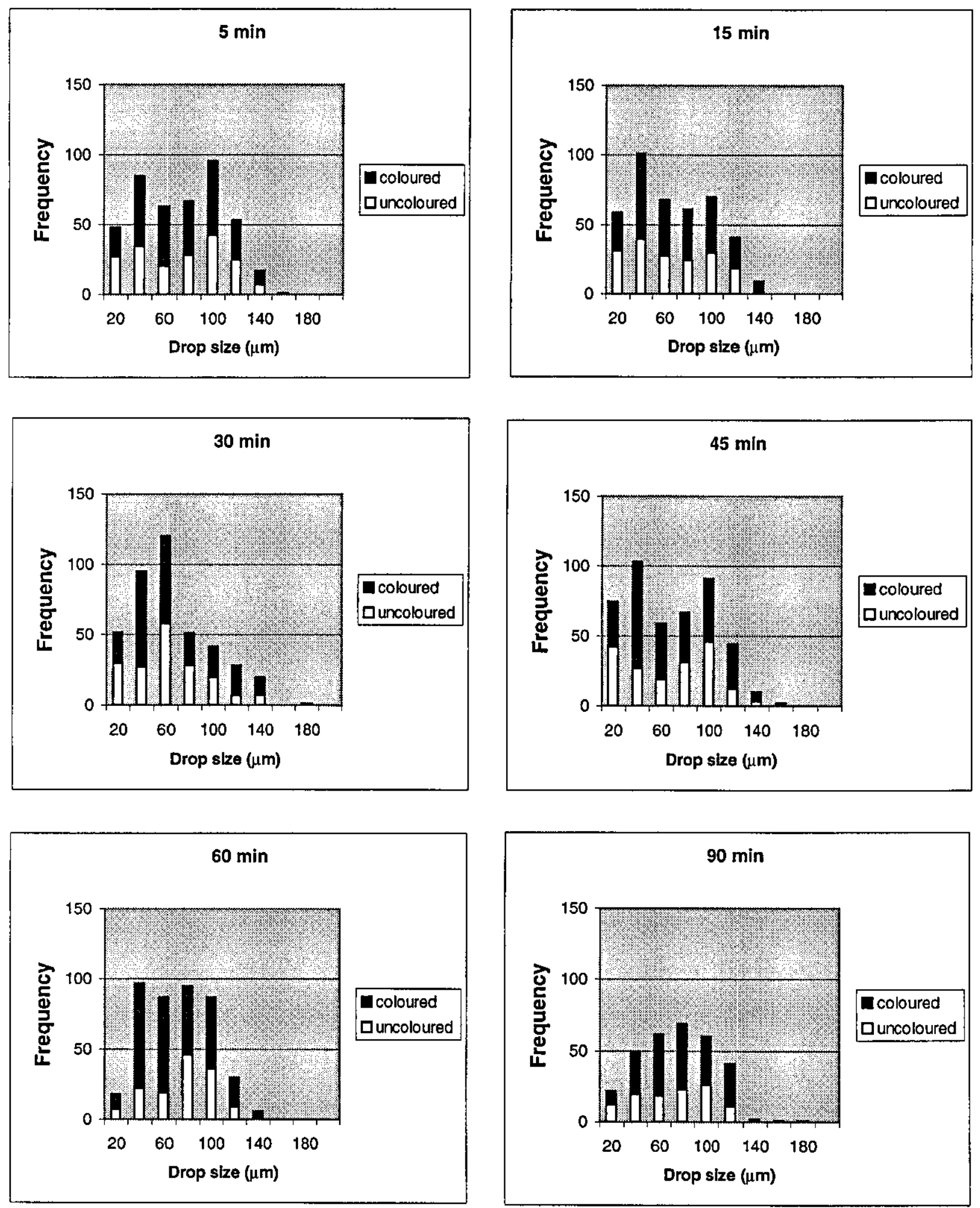

Figure 4.30: Drop size distribution for mixing of two stabilised dispersions of $10 \mathrm{wt} \%$ Polystyrene solution dispersed phase (continue on next page) 

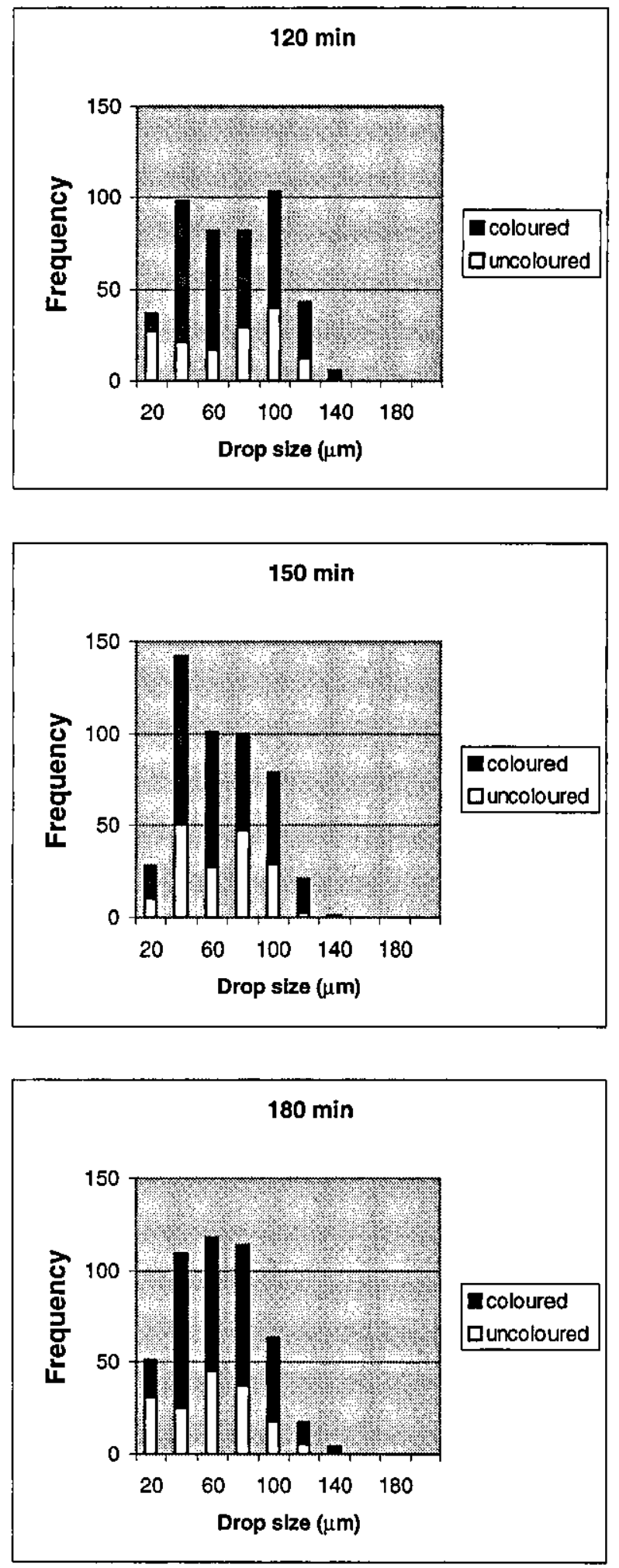

Figure 4.30 (continued) 


\subsubsection{Comparisons between dispersed mixing and batch mixing}

In this section, the drop mixing behaviour in disperse mixing and batch mixing are studied for comparison. In order to study this comparison, the experimental conditions for both dispersed mixing and batch mixing should be the same. It was noted that the experimental conditions for batch mixing in section 4.2 are not the same as in the dispersed mixing in section 4.3. Therefore new experiments have been designed for this purpose.

The new batch mixing experiments were designed such that the premixing dispersion with the same conditions as in dispersed mixing experiment were run for 120 minutes. At 120 minutes half of the total dispersion was drained out. Then, new dispersed phase and continuous phase were added to the remaining dispersion. Their amounts were the same as in the remaining dispersion. These two solutions were not premixed and they were added separately to the dispersion.

Figure 4.31 shows the comparison between extent of mixing for dispersed mixing and batch mixing for the dispersed phase of $0 \mathrm{wt} \% \mathrm{PS}+0 \mathrm{wt} \%$ PS. It can be seen that rate of mixing for batch mixing is higher than the dispersed mixing. This is because that the drops in the two stabilised dispersions are more protected by stabiliser compared to the batch mixing. In the batch mixing, the unstabilised batch are not protected and therefore mixed faster with the prestabilised dispersion. The results also show that at the beginning of the batch mixing, the rate of mixing is high and become slower later. It can be explained that at the beginning of batch mixing the added batches are not stabilised and after that they start to be stabilised when the stabiliser starts to adsorb on the surface of the drops. The transitional behaviour of the drop size distribution of the batch mixing of $0 \mathrm{wt}$ PS $\%+0 \mathrm{wt} \%$ PS is shown in figure 4.32. The results show that at the beginning, the drop size distribution is wide and then becomes less wide and remained the same. It can be explained that the stabiliser takes some time to adsorb on the drop surface. It can be seen that at 5 minutes there are significant amounts of large drops that are all coloured. These large coloured drops contribute to higher extent of mixing. This phenomenon also explains the higher rate of mixing at the beginning of batch mixing at this condition. 


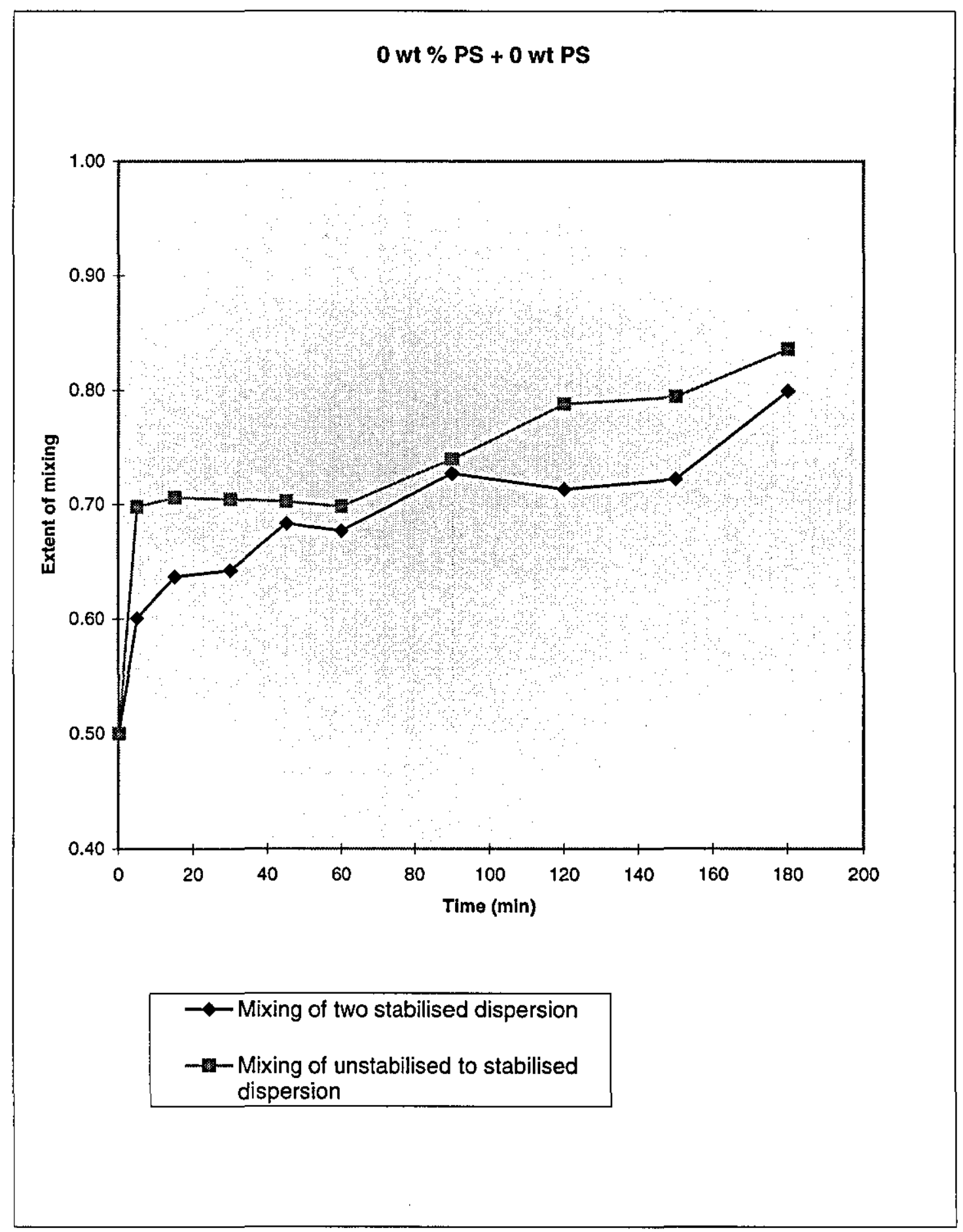

Figure 4.31: Extent of mixing for dispersed mixing and batch mixing ( $0 \mathrm{wt} \% \mathrm{PS}+0 \mathrm{wt}$ $\%$ PS) 

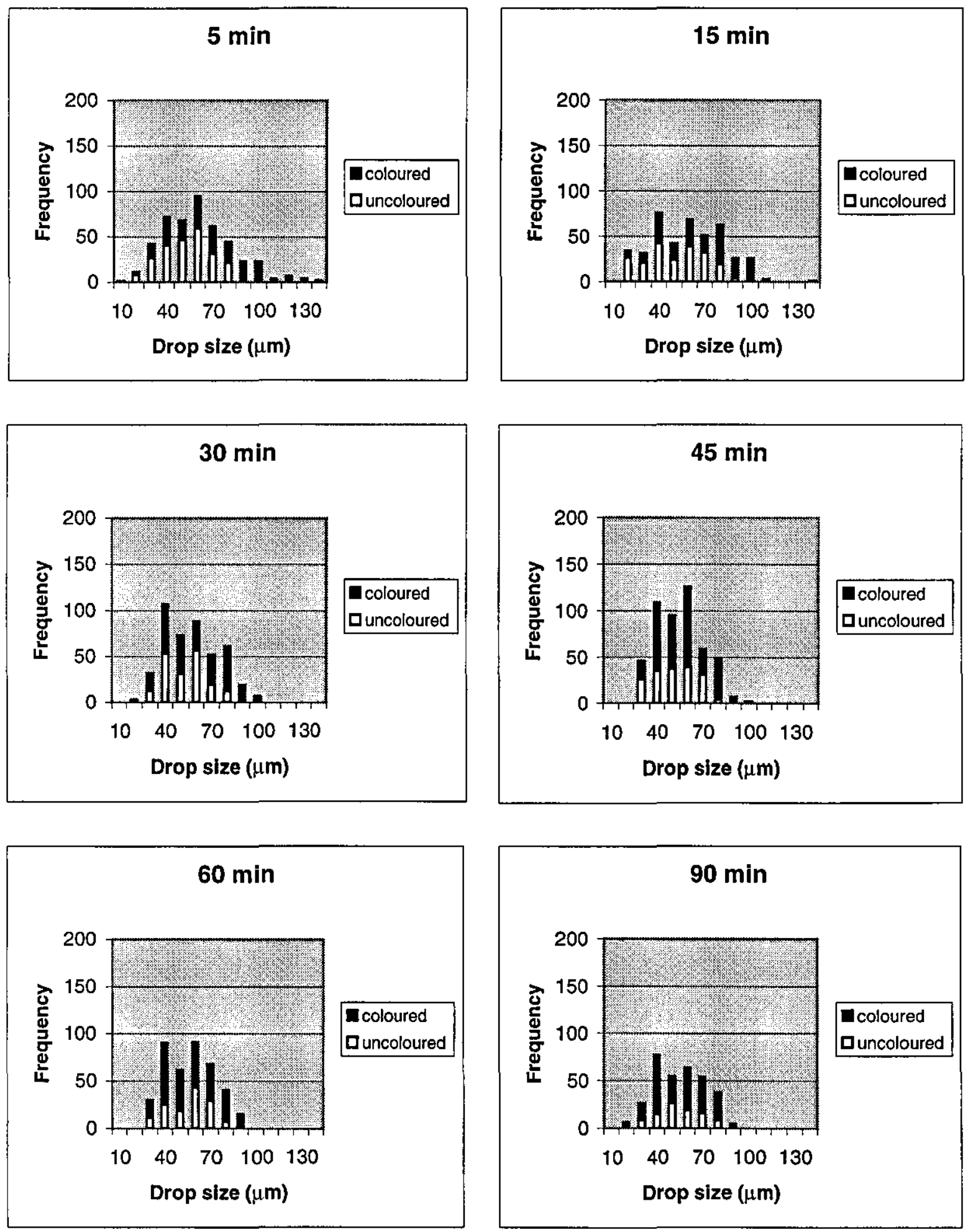

Figure 4.32: Drop size distribution for batch mixing of $0 \mathrm{wt} \%$ PS +0 wt $\%$ PS (continue on next page) 

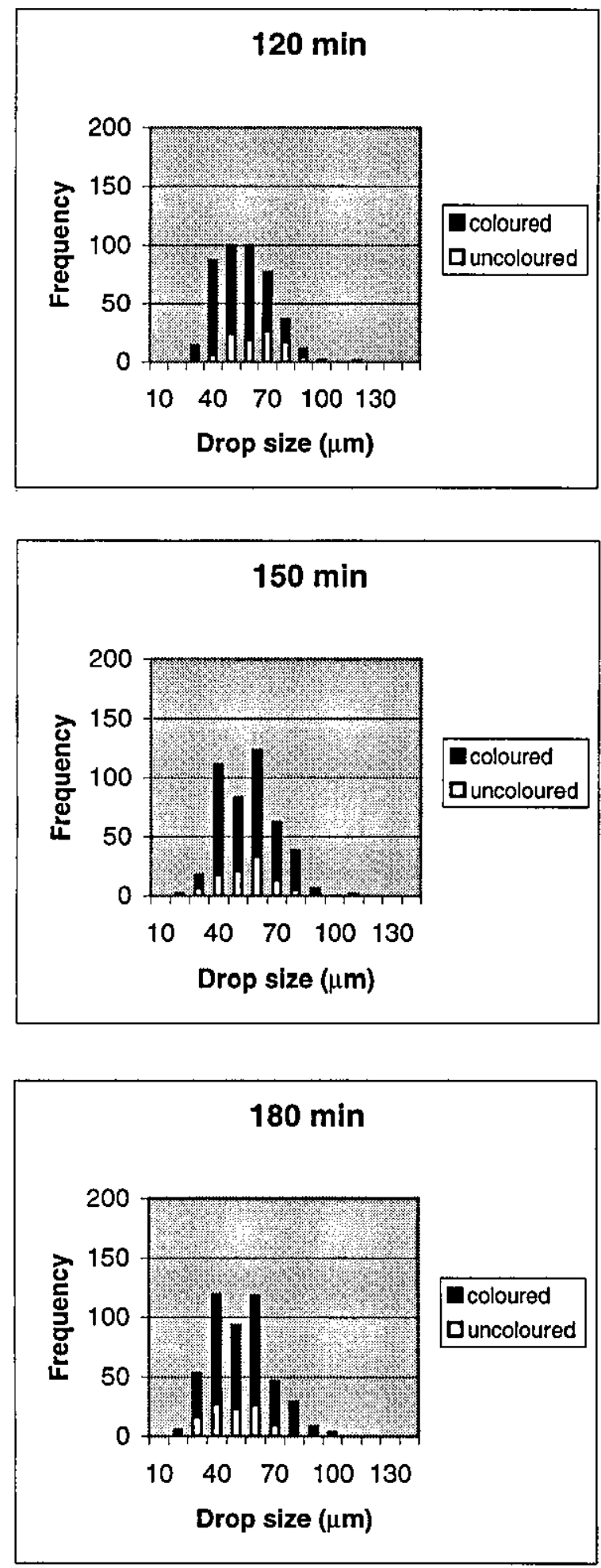

Figure 4.32 (continued) 
The comparison was also done for higher dispersed viscosity to study the effect of the viscosity. For this purpose, the batch mixing experiment was also run for of $10 \mathrm{wt} \%$ PS $\%+10 \mathrm{wt} \%$ PS. The comparison for this viscosity was shown in figure 4.33 . The results show that there is not much difference in the rate of mixing between the dispersed mixing and batch mixing. It can be explained that viscosity plays an important role in this mixing. For the batch mixing, even though the batch added is not protected by the stabiliser at the beginning, the viscosity of the added drops slows the mixing. Figure 4.34 shows the transitional behaviour of the drop size distribution for the batch mixing of $10 \mathrm{wt} \%+10 \mathrm{wt} \%$ PS of dispersed phase. The result shows that their drop size distributions are quite similar starting from 5 minutes to 180 minutes. It can also be seen that at the beginning, larger drops are not all coloured as in the $0 \mathrm{wt} \%+0 \mathrm{wt} \%$ PS of dispersed phase. Therefore it can be said that at higher viscosity of dispersed phase, the drop mixing behavior is quite similar for both dispersed mixing and batch mixing. 
$10 w t \%$ PS + 10 wt $\%$ PS

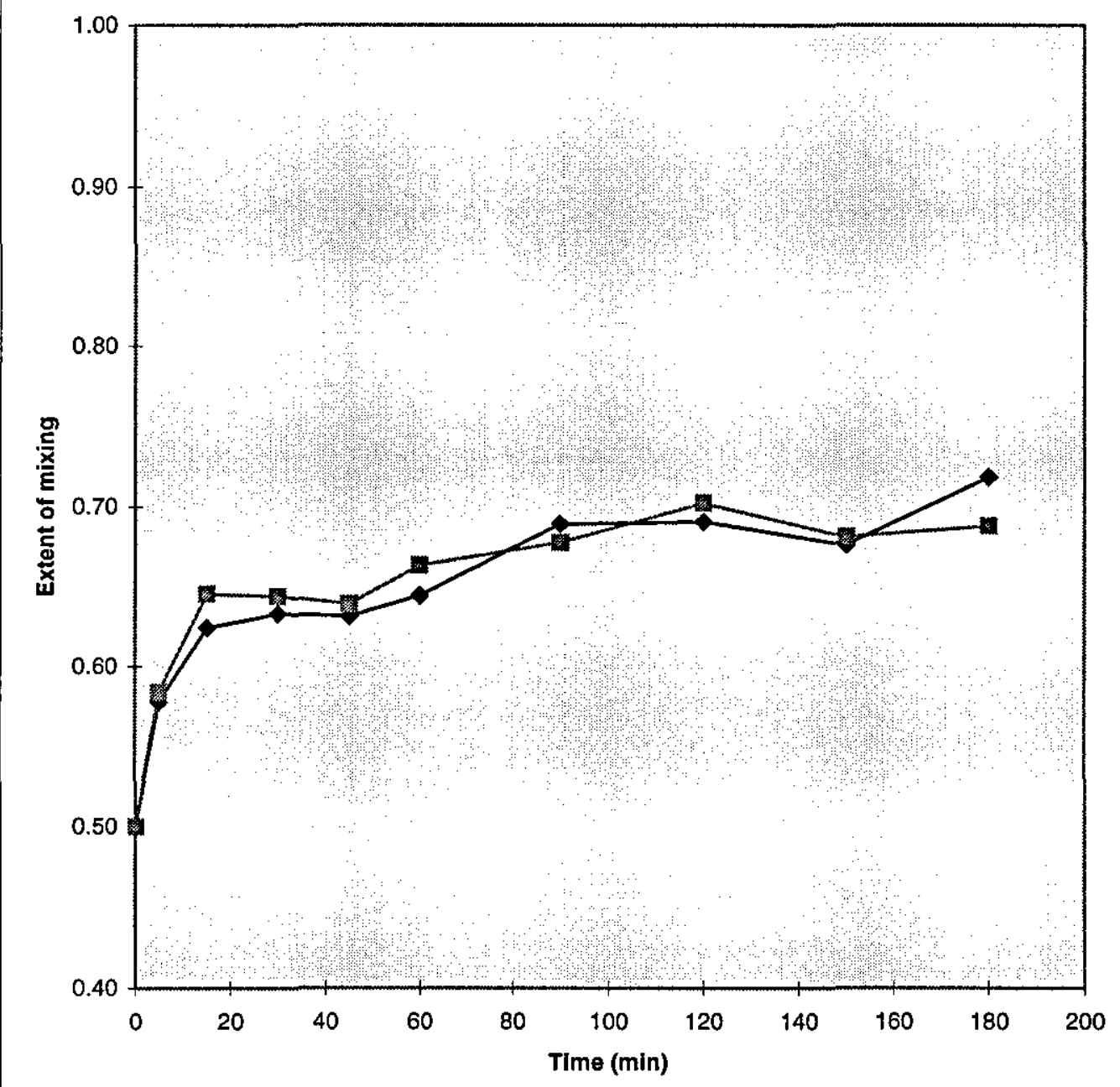

$\rightarrow$ Mixing of two stabilised dispersion

- -Mixing of unstabilised to stabilised dispersion

Figure 4.33: Extent of mixing for dispersed mixing and batch mixing (10 wt $\%$ PS +10 wt \% PS) 

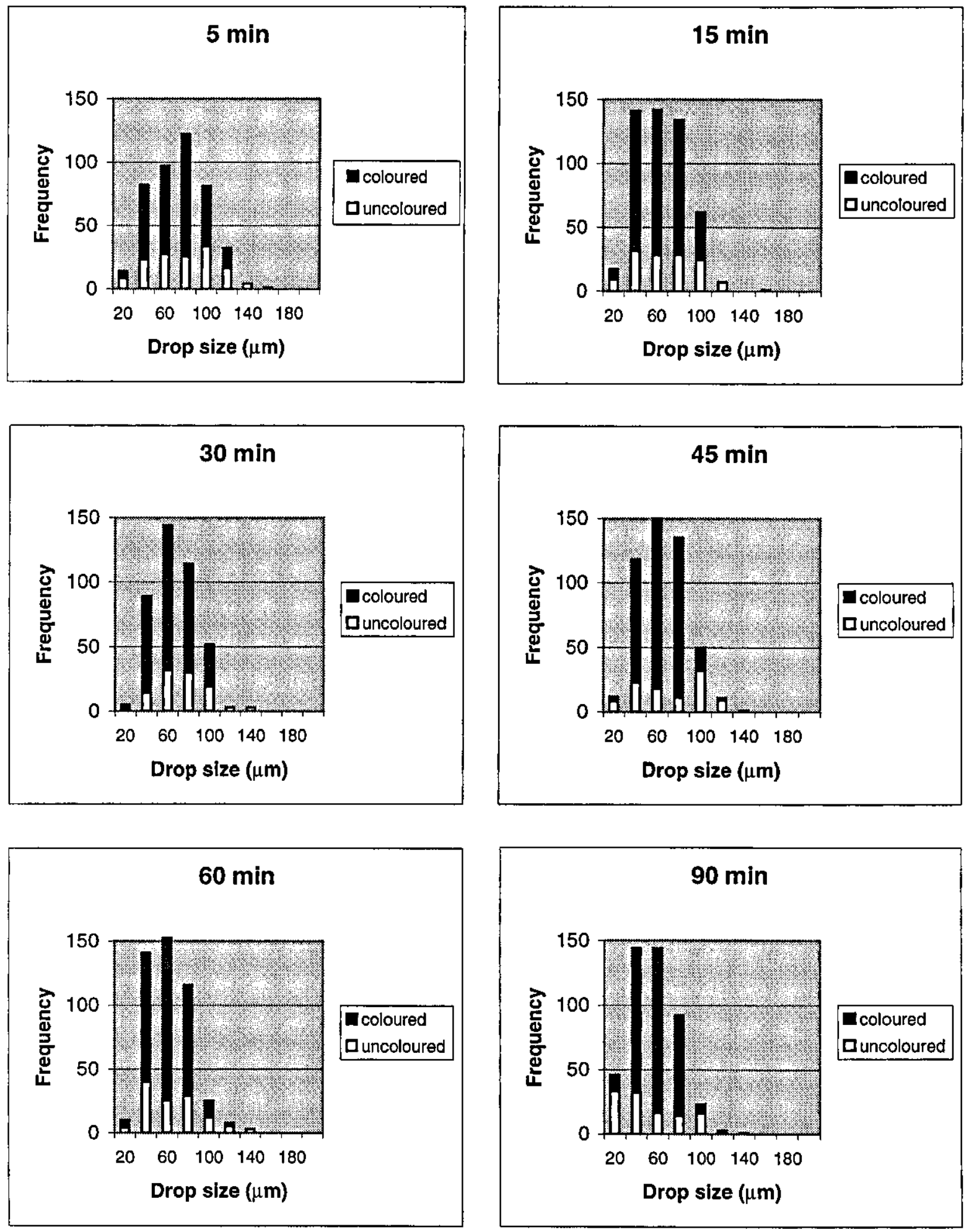

Figure 4.34: Drop size distribution for batch mixing of $10 \mathrm{wt} \%$ PS $+10 \mathrm{wt} \%$ PS (continue on next page) 

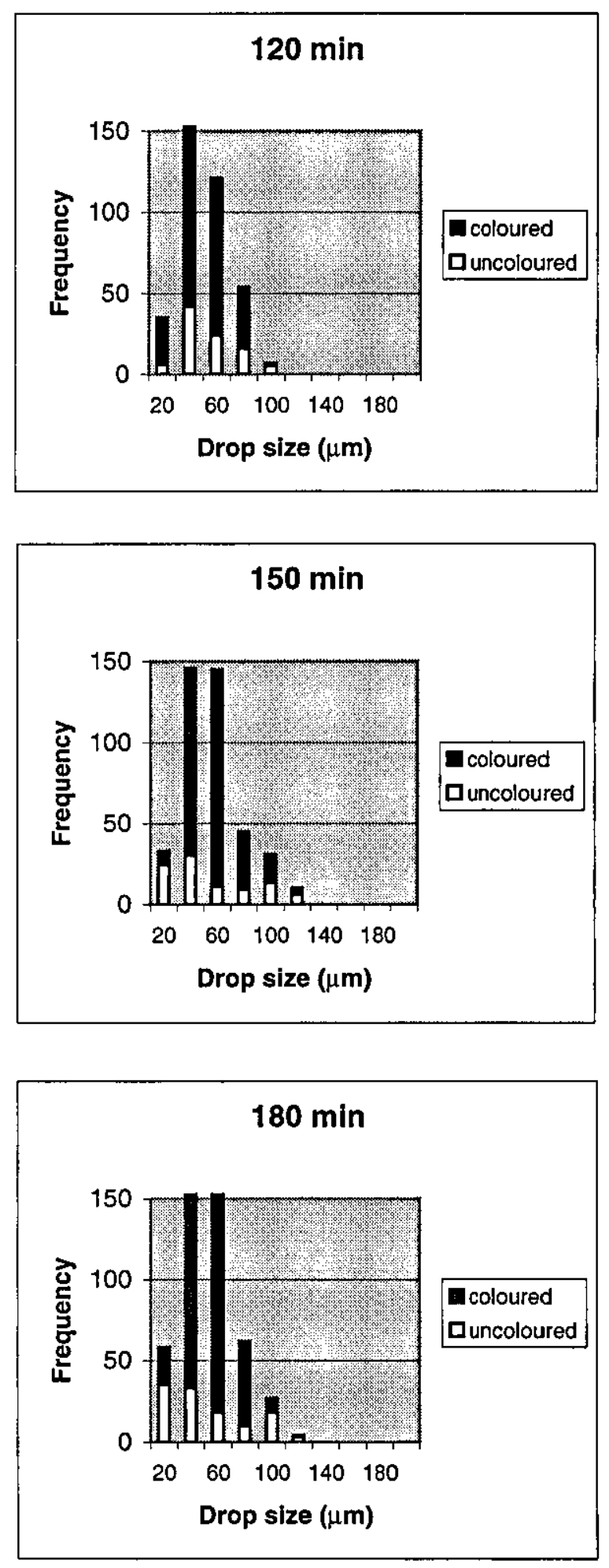

Figure 4.34 (continued) 


\subsubsection{MIXING OF DIFFERENT VISCOSITIES OF DISPERSED PHASES}

The purpose of this subsection was to study the effect of different viscosities of the dispersed phase of two dispersions on the rate of mixing. The method was the same as in section 4.3.1 except the dispersed phase of the two dispersions was different. Two sets of experiments were successfully run for this purpose. Following are the two sets of the experiments.

1) Mixing of $0 \mathrm{wt} \%$ polystyrene solution (coloured) and $5 \mathrm{wt} \%$ polystyrene solution (uncoloured)

2) Mixing of $0 \mathrm{wt} \%$ polystyrene solution (coloured) and $10 \mathrm{wt} \%$ polystyrene solution (uncoloured)

An attempt was also made to run the mixing of $0 \mathrm{wt} \%$ polystyrene and $15 \mathrm{wt} \%$ polystyrene, however a significant error occurred on the analysis of the drops since there were big differences between the drop size of $0 \mathrm{wt} \%$ polystyrene and $15 \mathrm{wt} \%$ polystyrene. Therefore these results were not produced for discussion.

Figure 4.35 shows the extent of mixing for two dispersions with two different viscosities of dispersed phase. The extent of mixing for mixing of two dispersions of $0 \mathrm{wt} \%$ polystyrene and $0 \mathrm{wt} \%$ polystyrene from previous results were also included in the figure for comparison purposes. The results shows that the mixing of $0 \mathrm{wt} \%$ polystyrene and $5 \mathrm{wt} \%$ polystyrene has a higher rate of mixing than the mixing of 0 wt $\%$ polystyrene and $10 \mathrm{wt} \%$ polystyrene. These results indicate that higher viscosity of dispersed phase (10 wt \% polystyrene) has reduced the mixing rate between the drops. As discussed previously, viscosity plays an important role to determine the rate of mixing at this condition.

Comparison was also made on the mixing of two dispersed phases with different viscosities and the mixing of two dispersed phases with identical viscosities. Figure 4.36 shows the extent of mixing for mixing of $0 \mathrm{wt} \%$ polystyrene and $5 \mathrm{wt} \%$ polystyrene, $0 \mathrm{wt} \%$ polystyrene and $0 \mathrm{wt} \%$ polystyrene, and $5 \mathrm{wt} \%$ polystyrene and $5 \mathrm{wt} \%$ polystyrene. It can be seen that the extent of mixing of $0 \mathrm{wt} \%$ polystyrene 


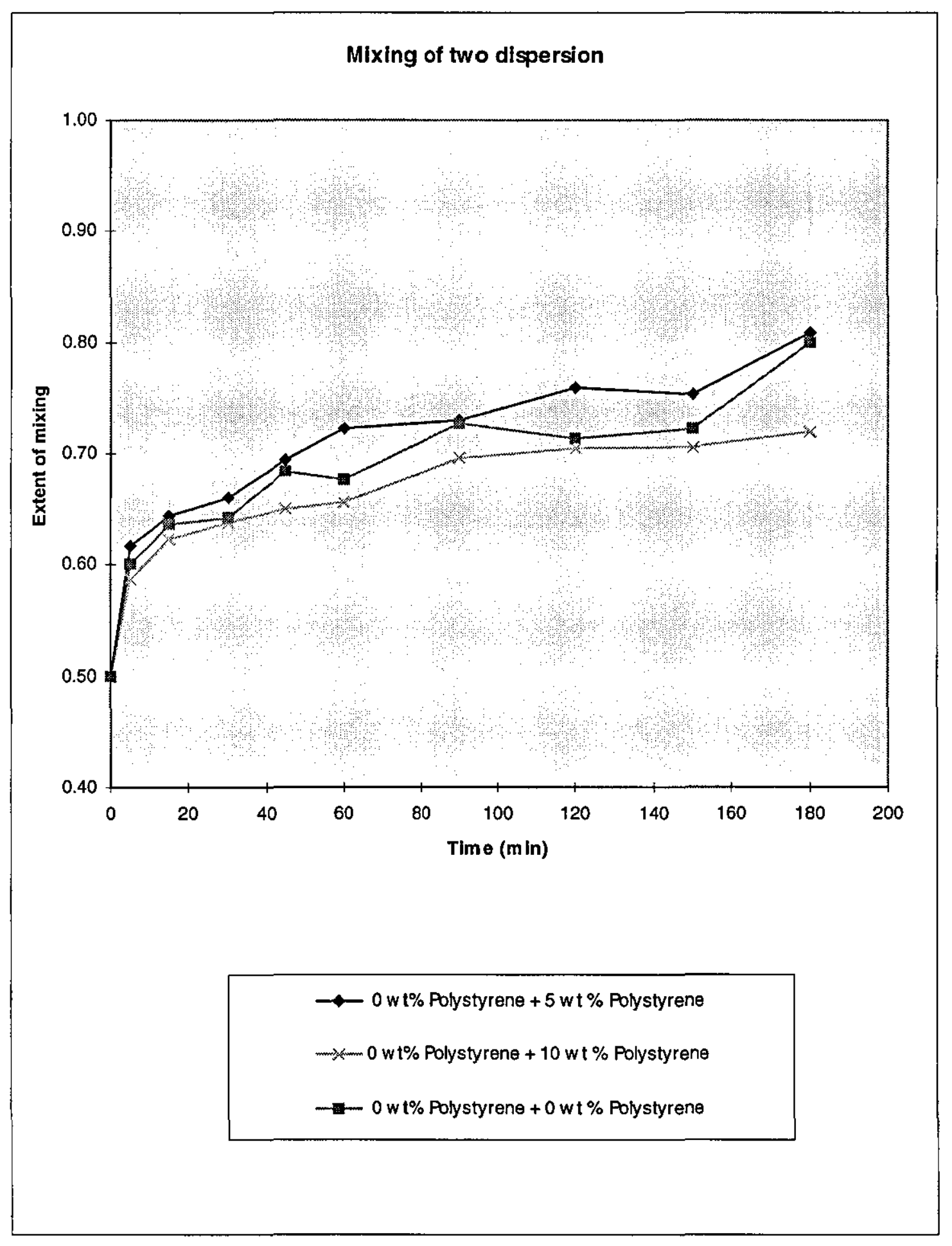

Figure 4.35: Extent of mixing for two dispersions of different viscosity of dispersed phase 


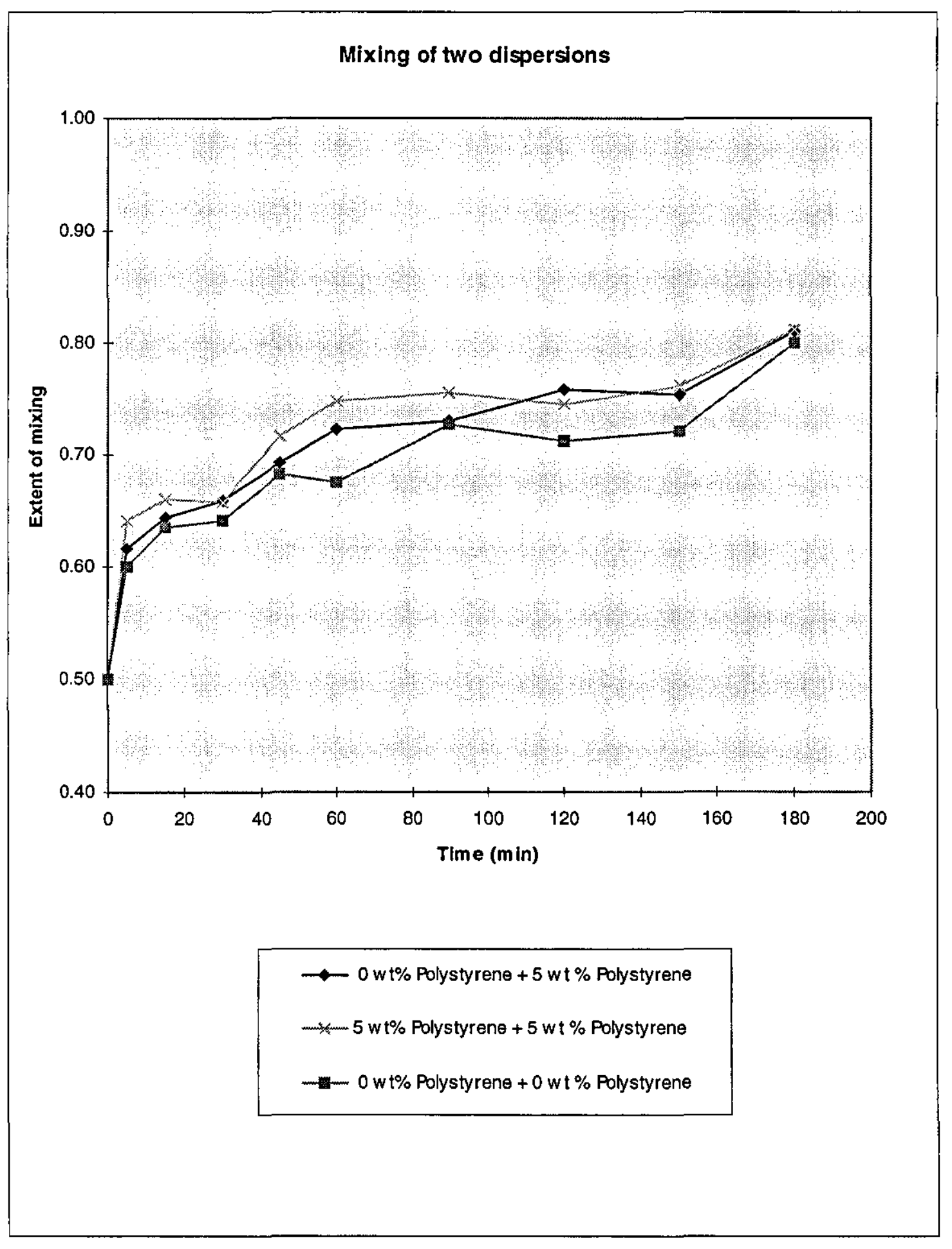

Figure 4.36: Extent of mixing for two dispersions of different viscosity of dispersed phase 
and $5 \mathrm{wt} \%$ polystyrene is generally located between the extent of mixing of $0 \mathrm{wt} \%$ polystyrene and $0 \mathrm{wt} \%$ polystyrene and the mixing of $5 \mathrm{wt} \%$ polystyrene and $5 \mathrm{wt} \%$ polystyrene. The increased extent of mixing was because of the increased of drop size which influenced the extent of mixing as discussed previously. Figure 4.37 shows the extent of mixing for mixing of $0 \mathrm{wt} \%$ polystyrene and $10 \mathrm{wt} \%$ polystyrene, 0 wt $\%$ polystyrene and $0 \mathrm{wt} \%$ polystyrene, and $10 \mathrm{wt} \%$ polystyrene and $10 \mathrm{wt} \%$ polystyrene. These results also show that the extent of mixing of $0 \mathrm{wt} \%$ polystyrene and $10 \mathrm{wt} \%$ polystyrene generally located between the extent of mixing of $0 \mathrm{wt} \%$ polystyrene and $0 \mathrm{wt} \%$ polystyrene and the mixing of $10 \mathrm{wt} \%$ polystyrene and $10 \mathrm{wt} \%$ polystyrene. The increased viscosity of one of the dispersions has affected the mixing rate to reduce.

Drop size analysis was also done to describe in detail the behaviour of the drop mixing in the mixing of two dispersions with different viscosities of dispersed phase. Figure 4.38 shows the transitional behavior of drop size distribution for the mixing of $0 \mathrm{wt} \%$ polystyrene and $5 \mathrm{wt} \%$ polystyrene. It can be seen that the drop size distribution at $5 \mathrm{~min}$ was wide and has a few drops of larger uncoloured drops (>90 $\mu \mathrm{m})$. The drop size distribution gradually become narrower with the larger drops vanishing. It can be clearly distinguished between the drop size distribution at 5 minutes and at 180 minutes. The drop size distribution at 180 minutes is narrower and there are no larger drops as at 5 minutes. These experiments show that the mixing occurred between the drops of different viscosities and resulted in drops with a new value of viscosity. This value must be between original viscosity of the drops before the coalescence occurred. It seemed that the decreased number of larger drops is because of the mixing with the lower viscosity drops. This coalescence will reduce the viscosity of the drops and therefore reduce the drop size. A similar situation occurred for the decreased number of smaller drops. The lower viscosity drops become higher in viscosity when they coalesce with higher viscosity drops. Therefore the drop size become larger. This drop mixing behavior explained why the drop size distribution became narrower as the mixing time increased.

The transitional behavior of drop size distribution for the mixing of 0 wt $\%$ polystyrene and $10 \mathrm{wt} \%$ polystyrene are shown in figure 4.39 . It can also be seen 
that the drop size distributions become narrower gradually from 5 minutes to 180 minutes. The transitional behaviour of the drop size distribution at this condition is similar to the mixing of $0 \mathrm{wt} \%$ polystyrene and $5 \mathrm{wt} \%$ polystyrene as discussed before. It was suggested by Kumar, S. et. al (1993) that when the difference of drop sizes is large, the coalescence efficiency will increase. However this effect didn't occur in the mixing of $0 \mathrm{wt} \%$ polystyrene and $10 \mathrm{wt} \%$ polystyrene even though the drop size difference was quite significant. This explains that, at this condition, viscosity has a more important effect than drop size difference. 


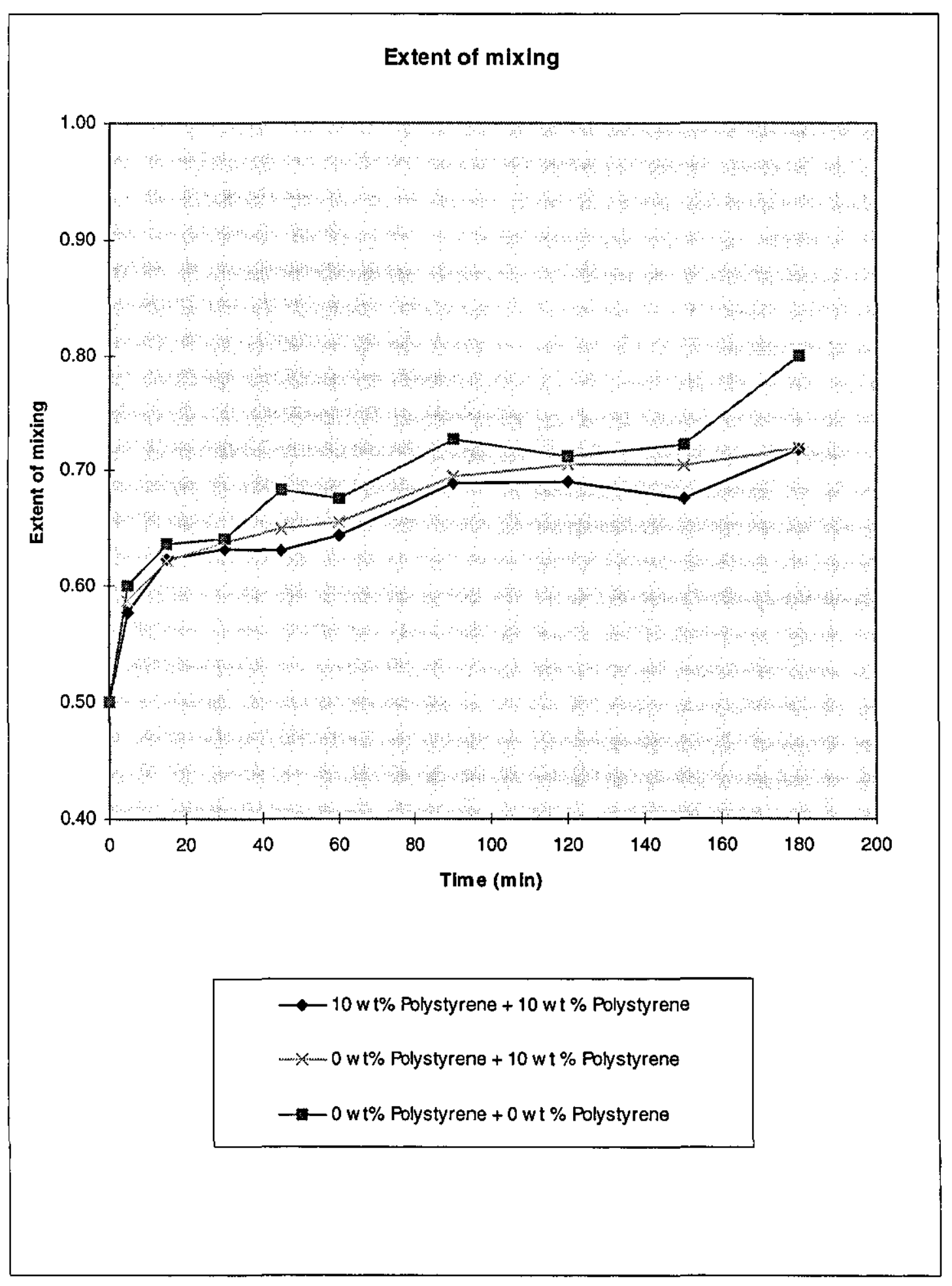

Figure 4.37: Extent of mixing for two dispersions of different viscosity of dispersed phase 

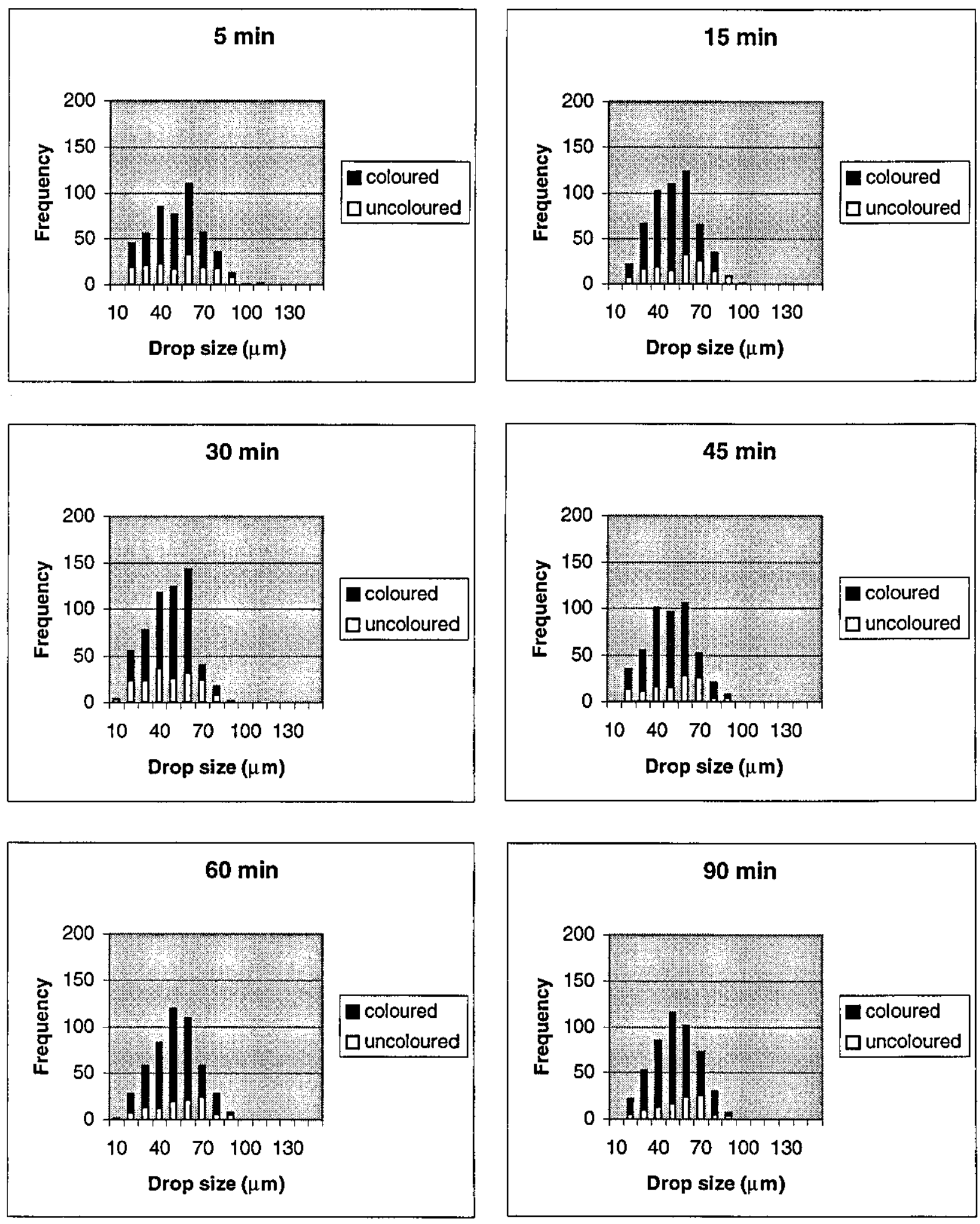

Figure 4.38: Drop size distribution for mixing of two stabilised dispersions (0 wt \% PS $+5 \mathrm{wt} \%$ PS)

(continue on next page) 

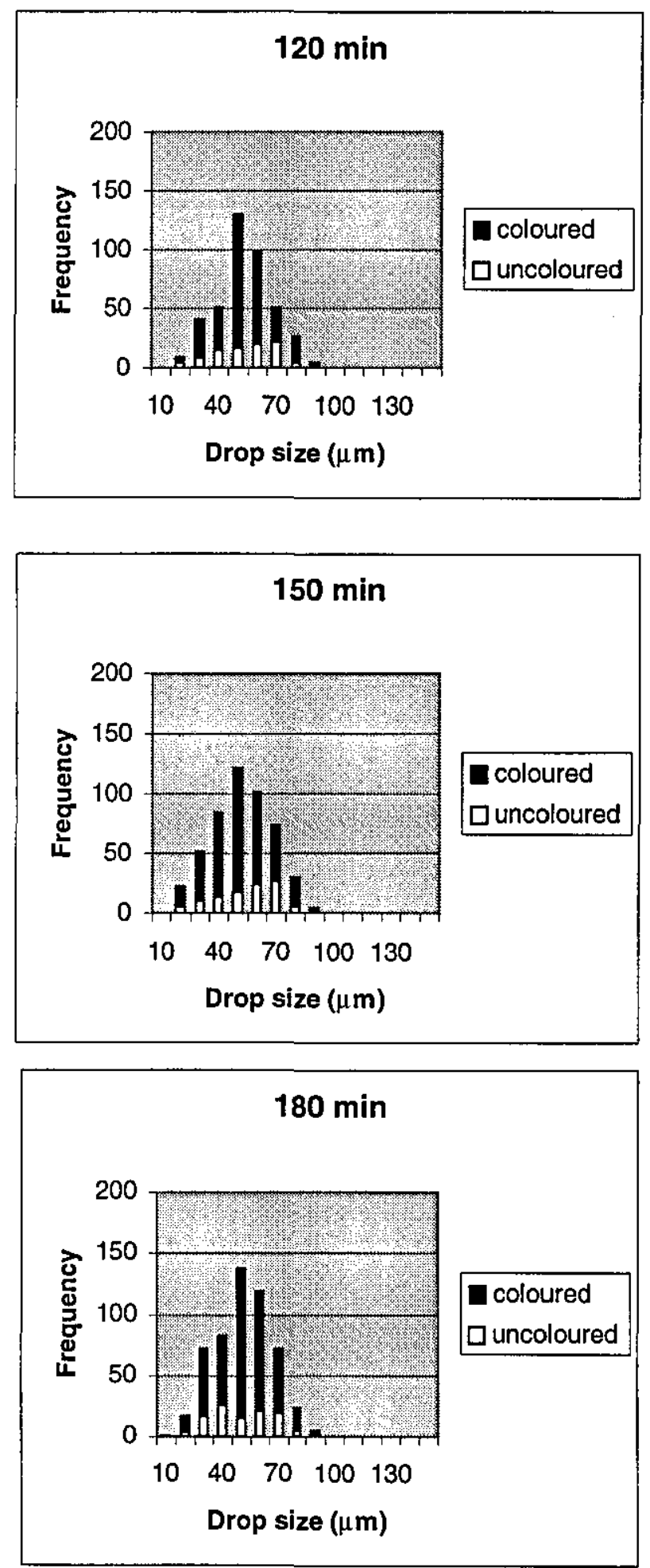

Figure 4.38 (continued) 

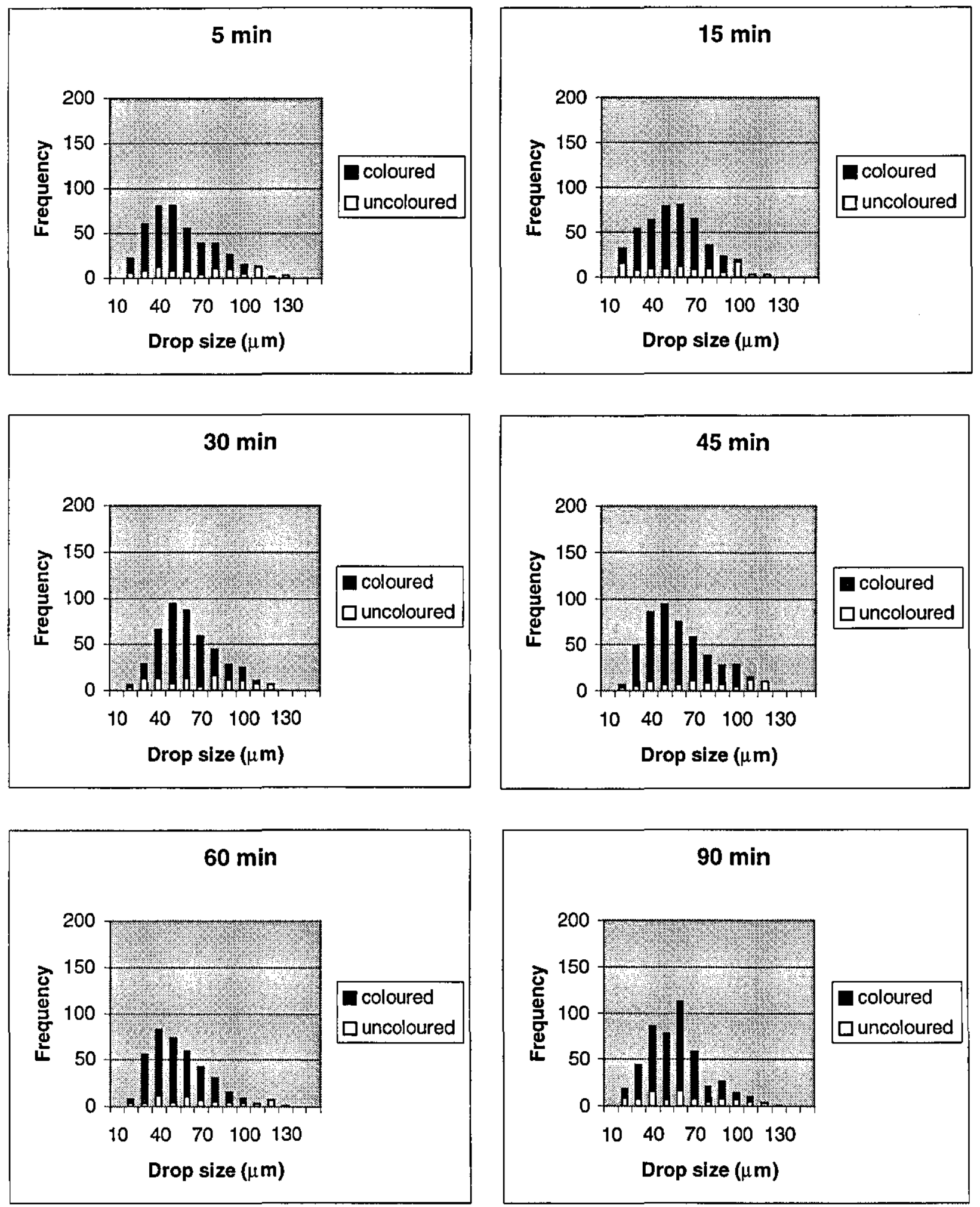

Figure 4.39: Drop size distribution for mixing of two stabilised dispersion (0 wt $\%$ PS + 0 wt \% PS)

(continue on next page) 

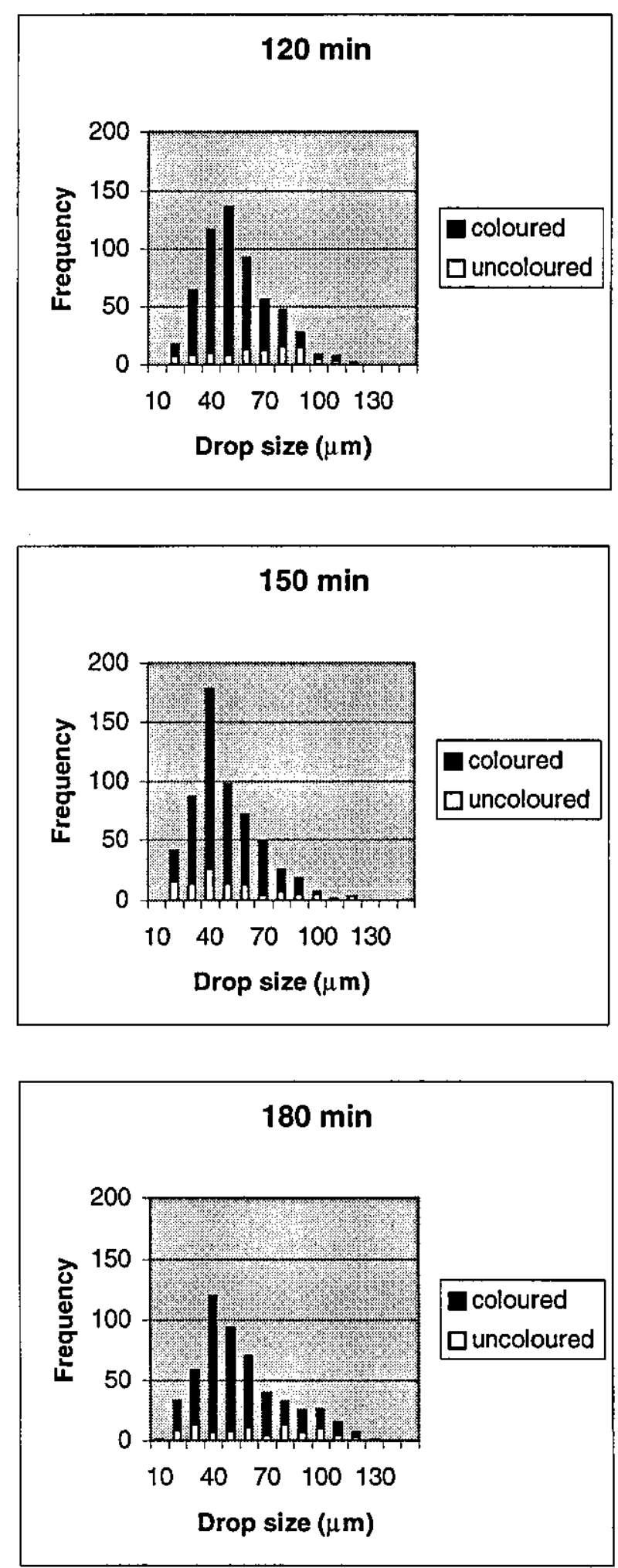

Figure 4.39: (continued) 


\section{CHAPTER 5}

\section{COMPARISON OF EXPERIMENTAL RESULTS WITH LITERATURE MODEL}

\subsection{Introduction}

In this section the drop mixing experimental results are compared with the prediction of previously derived models from literature for the coalescence rate. Therefore, coalescence rate data have to be derived from the extent of mixing data from the experimental results. Consideration of the coalescence rate results will only concentrate on the coalescence between coloured and uncoloured drops. Whereas, previous works from the literature generally were based on the overall coalescence of all drops. Therefore those models have some limitation for this application.

In these experiments, the coalescence rate between coloured and uncoloured drops is defined as

$F=$ volume of coloured drops coalescing with uncoloured drop per unit time.

This definition was based on the definition by Madden A.J. and Damerell G.L. (1962). In their studies, they assumed that all drops have equal volume. 


\subsection{Experimental Results}

For these experimental results, it is also assumed that all drops in the dispersion have the same volume. The experiments of mixing of two dispersions with the same viscosity were selected for this modeling study. These experiments were selected because they have the same property before and after the mixing. Futhermore the viscosity of each drop in the dispersion remains the same during the mixing. The overall average diameter during the dispersion was used as the constant drop size diameter. The drop volume for different viscosity of the dispersed phase are shown in Table 5.1 .

To calculate the coalescence rate from the experimental results, the data of extent of mixing were taken at 5 minute after the mixing of the two dispersions started. It is assumed that the coalescence rate is constant at the period during 0 to 5 minutes after the mixing started. This value is taken as the initial rate $\left(F_{0}\right)$. The calculated initial coalescence rate is as follows,

$$
F_{o}=\frac{\left(\mathrm{X}_{5 \mathrm{~min}}-\mathrm{X}_{0 \mathrm{~min}}\right) \times \text { Volume of dispersed phase }}{300 \mathrm{sec}}
$$

where $X=$ the extent of mixing

Extent of mixing at 0 minute is 0.5 for all different viscosities and volume of dispersed phase is $50 \mathrm{ml}$. The values of extent of mixing at 5 minutes and the initial coalescence rate for the different viscosity of the dispersed phase are shown in Table 5.1. Data for mixing of styrene and styrene dispersions are not included in this analysis since it was found that viscosity does not play an important role in the drop mixing behavior. Data for mixing of $0 \mathrm{wt} \% \mathrm{PS}+0 \mathrm{wt} \% \mathrm{PS}$ are not included in this analysis since the viscosity does not play an important role in the drop mixing behavior as observed in the last chapter. 
Table 5.1: Experimental data for overall average drop diameter, extent of mixing and initial coalescence frequency.

\begin{tabular}{|l|c|c|c|}
\hline $\begin{array}{c}\text { Concentration of } \\
\text { dispersed phase (mixing } \\
\text { of two dispersions) }\end{array}$ & $\begin{array}{c}\text { Overall average } \\
\text { drop diameter } \\
(\mu \mathrm{m})\end{array}$ & $\begin{array}{c}\text { Extent of } \\
\text { mixing }\end{array}$ & $\begin{array}{c}\text { Initial } \\
\text { coalescence } \\
\text { rate } \\
\left(\mathrm{cm}^{3} / \mathrm{s}\right)\end{array}$ \\
\hline $\begin{array}{l}5 \text { wt \% } \text { Polystyrene } \\
\text { solution }\end{array}$ & 51.4 & 0.641 & 0.0235 \\
\hline $\begin{array}{l}10 \text { wt \% Polystyrene } \\
\text { solution }\end{array}$ & 60.1 & 0.578 & 0.0125 \\
\hline $\begin{array}{l}15 \text { wt \% Polystyrene } \\
\text { solution }\end{array}$ & 89.6 & 0.503 & 0.000500 \\
\hline
\end{tabular}

\subsection{Coalescence rate model}

The model used for deriving coalescence rate will be based on the product of the collision rate and coalescence efficiency.

Coalescence rate $=$ collision rate $\mathrm{x}$ coalescence efficiency

This can be written in mathematical form as

$$
F=\theta x \exp \left(-\lambda_{c}\right)
$$

where

$\theta=$ collision rate (volume of drops colliding per unit time)

$\lambda_{c}=$ coalescence efficiency function 


\subsubsection{Collision rate}

The expression for the collision rate is derived by assuming that the mechanism of collision in a locally isotropic flow field is analogous to collision between molecules as in the kinetic theory of gases.

The expression of binary collision rate with correction factor is as follows:

$$
\omega_{c}=k_{c} \frac{\varepsilon^{1 / 3}}{(1+\phi)}\left(d_{x}{ }^{2}+d_{y}{ }^{2}\right) \sqrt{d_{x}^{2 / 3}+d_{y}{ }^{2 / 3}}
$$

$k_{c}=$ proportionality constant

$\varepsilon=$ energy dissipation

$\phi=$ volume fraction $=0.1$

$d_{x}, d_{y}=$ drop diameter (assume equal)

The value of $k_{c}$ is a universal constant and was taken from the modelling on polystyrene suspension polymerisation done by Alvarez et al (1994). They found that $k_{c}=1.37 \times 10^{-2}$.

For the energy dissipation, Rushton et al. (1950) showed that

$$
\begin{aligned}
\varepsilon= & \mathrm{k}_{\varepsilon} \mathrm{N}^{3} \mathrm{D}^{2} \\
N= & \text { stirring speed }=5.83 \mathrm{rps} \\
D= & \text { impeller diameter }=5 \mathrm{~cm} \\
k_{\varepsilon}= & \text { proportionality constant } \\
= & \mathrm{N}_{\mathrm{p}} \mathrm{V} / \mathrm{D}^{3} \\
& \mathrm{~V}=\text { Reactor Volume }=500 \mathrm{ml} \\
& \mathrm{N}_{\mathrm{p}}=\text { Power number }
\end{aligned}
$$

The power number was determined experimentally by many workers and was found to be in the range of 4-6 (Lee and Tasakorn, 1979; Nienow and Miles, 1971; Rushton et al, 1950). In this equation $N_{p}$ is assumed to be 5 (mean value). 
After substitution of all the values known, the collision rate of each different viscosity of the dispersed phase was determined. The number of total collision rate was determined by multiplying the collision rate by the (total number of drops $/ 2$ ). The total number of drops was determined by dividing the volume of dispersed phase by the drop volume. The result was shown in Table 5.2.

Table 5.2: Collision rate of different dispersed phase of mixing of two dispersions

\begin{tabular}{|c|c|}
\hline $\begin{array}{c}\text { Concentration of dispersed } \\
\text { phase (mixing of two } \\
\text { dispersions) }\end{array}$ & $\begin{array}{c}\text { Total collision rate } \\
\left(\mathrm{cm}^{3} / \mathrm{s}\right)\end{array}$ \\
\hline $5 \mathrm{wt} \%$ Polystyrene solution & 2615 \\
\hline $10 \mathrm{wt} \%$ Polystyrene solution & 2354 \\
\hline $15 \mathrm{wt} \%$ Polystyrene solution & 1805 \\
\hline
\end{tabular}

\subsubsection{Coalescence efficiency}

The model used for coalescence efficiency is the model that was developed by Alvarez et al. (1994). This model considered the effect of drop viscoelasticity, surface tension and film drainage.

The model was derived as follows:

$$
\begin{aligned}
& \lambda_{c}(x, y)=a_{c} \Omega\left(d_{x y}\right)-b_{c} / W e_{d}\left(d_{f}\right) \\
& a_{c} \text { and } b_{c} \text { are proportionality constants }
\end{aligned}
$$




$$
\begin{gathered}
\Omega\left(d_{x y}\right)=\frac{6}{\operatorname{Re}\left(d_{x y}\right)\left[1+\operatorname{Re}\left(d_{x y}\right) V_{e}\left(d_{x y}\right)\right]}+\frac{1}{W e\left(d_{x y}\right)} \\
d_{x y}=\text { mean length for deformation in coalescence interpenetration } \\
=\left(d_{x}^{-1}+d_{y}^{-1}\right)^{-1}
\end{gathered}
$$

It is assumed that all diameters of the drops in the dispersion were of equal size. Therefore

$$
d_{x}=d_{y}
$$

The values of drop diameter are as shown in Table 5.1.

For the drop Reynold number

$$
\operatorname{Re}\left(d_{x y}\right)=d_{x y} \frac{u\left(d_{x y}\right)}{v_{s}}
$$

$u\left(d_{x y}\right)$ is mean turbulent velocity and was determined by the meansquared turbulent velocity. Correction factor is introduced in this equation .

$$
u^{2}\left(d_{x y}\right)=k_{v}\left(\varepsilon d_{x y}\right)^{2 / 3} /(1+\phi)^{2}
$$

$k_{v}$ is a proportional constant and related to the universal constant in the expression for drop break-up rate

$$
k_{b}=k_{v}{ }^{1 / 2}
$$

The value of $k_{v}$ is equal to 0.73 according to Alvarez et al (1994)

By using all the known data, mean turbulent velocity can be determined. 
The kinematic viscosity of the dispersed phase $\left(V_{s}\right)$ was determined from density and viscosity data as in chapter 3 . The values of kinematic viscosity are shown in Table 5.3

Table 5.3: Kinematic viscosity of suspension for different viscosity of dispersed phase

\begin{tabular}{|l|c|}
\hline $\begin{array}{l}\text { Concentration of dispersed phase } \\
\text { (mixing of two dispersions) }\end{array}$ & $\begin{array}{c}\text { Kinematic viscosity } \\
\left(\mathrm{cm}^{2} / \mathrm{s}\right)\end{array}$ \\
\hline $5 \mathrm{wt} \%$ Polystyrene solution & 0.0830 \\
\hline $10 \mathrm{wt} \%$ Polystyrene solution & 0.340 \\
\hline $15 \mathrm{wt} \%$ Polystyrene solution & 1.13 \\
\hline
\end{tabular}

Therefore the drop Reynolds number can be determined and the values are shown in table 5.5.

For drop Weber number the equation is given as follows:

$$
\mathrm{We}\left(d_{x y}\right)=\rho_{d} d_{x y} \frac{u^{2}\left(d_{x y}\right)}{\sigma}
$$

The variable that needs to be determined in this equation is the interfacial tension. The interfacial tension was determined from experiments and the details of the determination are given in the appendix. The values were based on the surfactant coverage on the drop phase.

The values of interfacial tension that were determined from experiments are shown in the following table. 
Table 5.4: Interfacial tension of the drops in the different viscosity of disperse phase.

\begin{tabular}{|l|c|}
\hline $\begin{array}{l}\text { Concentration of dispersed phase } \\
\text { (mixing of two dispersions) }\end{array}$ & $\begin{array}{c}\text { Interfacial Tension } \\
(\mathrm{N} / \mathrm{cm})\end{array}$ \\
\hline 0 wt \% Polystyrene & $2.35 \times 10^{-4}$ \\
\hline $5 \mathrm{wt} \%$ Polystyrene solution & $2.36 \times 10^{-4}$ \\
\hline $10 \mathrm{wt} \%$ Polystyrene solution & $2.22 \times 10^{-4}$ \\
\hline $15 \mathrm{wt} \%$ Polystyrene solution & $1.75 \times 10^{-4}$ \\
\hline
\end{tabular}

The values of drop Weber number were then determined. The results are shown in Table 5.5.

Table 5.5: Drop Reynolds number and drop Weber number for different viscosity of dispersed phase.

\begin{tabular}{|c|c|c|}
\hline $\begin{array}{c}\text { Concentration of dispersed } \\
\text { phase (mixing of two } \\
\text { dispersions) }\end{array}$ & $\begin{array}{c}\text { Drop Reynolds } \\
\text { Number }\end{array}$ & $\begin{array}{c}\text { Drop Weber } \\
\text { Number }\end{array}$ \\
\hline 5 wt \% Polystyrene solution & 0.164 & 277 \\
\hline $10 \mathrm{wt} \%$ Polystyrene solution & 0.049 & 386 \\
\hline $15 \mathrm{wt} \%$ Polystyrene solution & 0.0253 & 957 \\
\hline
\end{tabular}

For transient viscoelasticity effect dimensionless number

$$
V_{e}\left(d_{x y}\right)=\Theta \exp \left(-\frac{1-\alpha}{2 Y_{o} \operatorname{Re}}\right)-\frac{1}{12}
$$

where $\alpha=\sqrt{1-48 Y_{o}}$

$$
\Theta=\frac{Y_{o}}{\alpha(1-\alpha)}\left[1+\alpha-\frac{(1-\alpha)^{2}}{1+\alpha}\right] \exp \left(-\frac{1-\alpha}{2 Y_{o} \operatorname{Re}}\right)
$$


and

$$
Y_{0}=\frac{\mu_{d}{ }^{2}}{\rho_{d} E_{d} d_{x y}{ }^{2}}
$$

where

$$
E_{d}=\chi E_{p}
$$

The value of elastic modulus for pure polystyrene is taken from Nielson L.E. (1974) which is $9 \times 10^{13} \mathrm{~g} / \mathrm{cm} \mathrm{min}^{2}$. Then the values of $Y_{o}$ are calculated and are shown in Table 5.6.

Table 5.6: Values of $Y_{o}$ for different concentration of dispersed phase

\begin{tabular}{|c|c|}
\hline $\begin{array}{c}\text { Concentration of dispersed } \\
\text { phase (mixing of two } \\
\text { dispersions) }\end{array}$ & $Y_{o}$ \\
\hline $5 \mathrm{wt} \%$ Polystyrene solution & $9.71 \times 10^{-7}$ \\
\hline $10 \mathrm{wt} \%$ Polystyrene solution & $3.54 \times 10^{-7}$ \\
\hline $15 \mathrm{wt} \%$ Polystyrene solution & $1.06 \times 10^{-7}$ \\
\hline
\end{tabular}

Therefore values of $V_{e}\left(d_{x y}\right)$ and $\Omega\left(d_{x y}\right)$ can be determined.

The next variable needed to be determined was the film drainage Weber number $\left(W e_{d}\right)$

$$
W e_{d}=\rho_{w} d_{f} u^{2}\left(d_{f}\right) / \sigma
$$

where

$$
\begin{aligned}
& d_{f}=\left(d_{x}{ }^{3}+d_{y}{ }^{3}\right) / d_{x y}{ }^{2} \\
& \rho_{w}=\text { water density }
\end{aligned}
$$

All the data in the above equation were known and therefore the film drainage number can be determined. Table 5.7 shows the results of the film drainage Weber number. 
Table 5.7: The film drainage Weber number for different viscosity of dispersed phase

\begin{tabular}{|c|c|}
\hline $\begin{array}{c}\text { Concentration of dispersed } \\
\text { phase (mixing of two } \\
\text { dispersions) }\end{array}$ & $\begin{array}{c}\text { Film drainage } \\
\text { Weber number }\end{array}$ \\
\hline $5 \mathrm{wt} \%$ Polystyrene solution & $4.44 \times 10^{4}$ \\
\hline $10 \mathrm{wt} \%$ Polystyrene solution & $1.12 \times 10^{5}$ \\
\hline $15 \mathrm{wt} \%$ Polystyrene solution & $3.83 \times 10^{5}$ \\
\hline
\end{tabular}

For the determination of the proportionality constants of $a_{c}$ and $b_{c}$, the values have to be determined from two set of experiments. The experiments with 5 wt $\%$ Polystyrene solution and $15 \mathrm{wt} \%$ Polystyrene solution were chosen for this purpose. Then the model can be used to predict the initial coalescence rate for the experiment $10 \mathrm{wt} \%$ Polystyrene for comparison with the experimental data. The proportionality constants were found as follows:

$$
\begin{aligned}
& a_{c}=5.17 \times 10^{-2} \\
& b_{c}=-1.89 \times 10^{5}
\end{aligned}
$$

Table 5.8 shows the comparison of the model prediction with experimental data from the experiment using $10 \mathrm{wt} \%$ Polystyrene.

Table 5.8: Comparison of the result of the model and experiment for $10 \mathrm{wt} \%$ Polystyrene

\begin{tabular}{|l|c|}
\hline & Extent of mixing \\
\hline Experimental results & 0.575 \\
\hline Predicted by model & 0.524 \\
\hline
\end{tabular}


The difference between of the result of experiment and the model prediction occurs due to assumptions made in the model, especially that all drops have the same size.

This model is not capable of predicting the extent of mixing between the coloured and uncoloured drops after the initial period of coalescence. This is due to the fact that after the extent of mixing of 0.5 , drops have different probabilities of collision between coloured and uncoloured drops. Volumes of coloured drops are increasing while volume of uncoloured drops are decreasing.

However this technique of dealing with the experiments can be used to determine the coalescence rate of all drops in the dispersion. It is assumed that the initial colescence rate has the same value as the overall coalescence rate of the dispersion. Since the drop size distribution in the experiments of mixing of two dispersions shows that they have similar size distributions at all time as shown in section 4.3 , the coalescence rate must be the constant. The coalescence rate and breakage rate is also then equal because the dispersion is at steady state. 


\section{CHAPTER 6}

\section{CONCLUSION AND RECOMMENDATION}

\subsection{CONCLUSION}

This research project has been performed with the aim to investigate the behaviour of drop mixing when new material is added to the existing suspension polymerisation. The study focuses on the effect of the dispersed phase viscosity on the drop mixing.

This work can be divided into three sections, first is the preliminary studies, second is the mixing of an unstabilised batch of monomer with the existing dispersion and third the mixing of two stabilised dispersions. The major results and achievements are summarised as follows.

\section{A. Preliminary Studies}

The experiments of added batches of toluene to a stabilised dispersion of polystyrene /toluene solution revealed that the coalescence rate of lower viscosity dispersed phases was higher. While for mixing of two dispersions, the results show that Tween 20 protects the drops more efficiently than PVA based on weight basis. It 
also revealed that PVA produced a narrower drop size distribution than Tween 20 . Therefore, this preliminary investigation suggested that viscosity of the dispersed phase and type of surfactant used play an important on the coalescence rate between the new material and the old existing drops.

B. Batch mixing of monomer with existing stabilised dispersion

The first part of the investigation was to observe the behaviour of the liquidliquid dispersion before the addition of a batch. The results revealed that for the three different viscosities of dispersed phase, the drop size initially decreased and then became more or less constant. The studies also revealed that for higher dispersed phase viscosity, a wider drop size distribution was produced.

The second part was the addition of a new batch of styrene to the existing dispersion of polystyrene/styrene solution. At higher agitation speed $(500 \mathrm{rpm})$, the result revealed that the coalescence rate increased as the viscosity of the dispersed phase increased. While at lower agitation speed $(350 \mathrm{rpm})$, the coalescence rate occurred more slowly with higher dispersed phase viscosity. From the detailed analysis on drop size, it can be summarised that drop size also plays an important role in determining the coalescence rate between the drops. At higher agitation speed, the mixing rate between higher viscosity dispersed phase and new added styrene is higher due to the larger drop size in the dispersion. The smaller droplets seemed relatively stable toward coalescence. The transitional behaviour of drop size distribution also shows that the new drops of styrene mixed with larger drops first then followed by smaller drops.

For mixing a batch styrene with the existing dispersion at lower agitation speed (350 $\mathrm{rpm}$ ) the extent of mixing increased relatively slowly compared with the batch mixing at higher speed. The studies suggest that at larger drop size, with increasing drop viscosity the coalescence efficiency became lower. From the analysis, it can be seen that a critical drop size exists which determines the coalescence efficiency effect. Above the critical drop size, mixing rate increases as the drop viscosity decreases. While below the critical drop size, drop size of the dispersion determines the coalescence rate; as the drop size increased, coalescence rate will also increase. The 
transitional behavior of drop size distribution for slower agitation speed ( $350 \mathrm{rpm}$ ) revealed that the drops coalesce at the middle range of the drop size, the larger size of drops seemed to be very stable toward coalescence.

The comparison of the behavior of drops mixing at different agitation speed was also studied. The results shows that as the stirring speed increased, the rate of coalescence also increased. On the studies of effect of concentration of PVA, the higher concentration of PVA used resulted in lower drop size. At $0.05 \mathrm{wt} \%$ PVA, drop size plays an important role in determining the drop mixing, a higher viscosity of dispersed phase has a higher coalescence rate. It also revealed that the batch mixing using a higher concentration of PVA has a slower coalescence rate.

The investigation of the effect of suspending agent shows that Tween 20 is much more efficient in stabilising and protecting the drops, based on a weight basis, as the coalescence rate is relatively low compared to PVA. The analysis on drop size shows that the dispersion using Tween 20 produced higher drop size and wider drop size distribution compared to the dispersion using PVA.

\section{Mixing of two dispersions}

Two types of mixing of dispersions were done in this project. First was the mixing of two stabilised dispersions of same viscosities of the dispersed phase. Second was the mixing of two stabilised dispersions with different viscosities of the dispersed phase.

For the first part, the results confirm that at larger drop size, with increasing drop viscosity, the coalescence efficiency become lower. The transitional behavior of drop size distribution shows that from the start of the mixing, their drop size distribution is similar. These revealed that the dispersion is at steady state during the mixing process.

The second part of mixing two stabilised dispersions also reveal that the higher viscosity of dispersed phase reduced the mixing rate between drops. The transitional behavior of drop size distribution shows that the drop size distribution gradually 
becomes narrower with the larger drops disappearing. It shows that the mixing occurred between the drops of different viscosities and resulted in drops with new value of viscosity. As a result, the coalescence reduces the viscosity of the drops and therefore reduces the drop size.

The comparison between batch mixing and mixing of two dispersions shows that the rate of mixing for the batch mixing is higher. The results also reveal that at the beginning of the batch mixing, the rate of mixing is initially high and becomes slower later. The drop size distribution also shows that at the beginning, it was wide and then becomes less wide and then remained the same.

D. Comparison of experimental results with literature model

The drop mixing experimental results are compared with the prediction of a previously derived model from literature. The model used was the one developed by Alvarez et al (1994). The model predicts the initial coalescence rate of mixing of two dispersions which is relatively close to the experimental results. This model can be used in this study to predict the initial coalescence rate. 


\subsection{RECOMMENDATION}

For further investigation, work might be done on the following aspects:

\section{1- Critical Drop Size}

As suggested from the experiments of batch mixing and mixing of two dispersions, a critical drop size occurs which determines the role of viscosity in the coalescence process. Therefore work can be done to determine the value of the critical drop size at certain viscosity of the dispersed phase.

\section{2- Several added batches of monomer to stabilised dispersion}

The method of addition of monomer to the stabilised dispersion can be programmed at different times. This investigation will study the effect of the programmed added batches of monomer on the drop mixing behavior.

3- Continuous adding of monomer to stabilised dispersion

This work will study the effect of the continuous adding of monomer to the stabilised dispersion on the drops mixing behavior. Then the methods of addition of monomer to the stabilised dispersion can be compared with each other.

\section{4- Polymerisation experiments}

Since this project used simulated dispersion for a polymerisation process, the result only predicts the behavior of drop mixing in a polymerisation process. Therefore a real polymerisation process can be performed in the future work for more accurate behavior of drop mixing. Experiments can be performed both for homopolymerisation and copolymerisation. 


\section{Correlation of viscosity with drop size}

Since this work focused mainly on the coalescence between added new material and existing dispersion, most attention was given to correlations for coalescence. The work can be extended to include the analysis of correlation on drop size with the viscosity of the dispersed phase. The Sauter mean diameter correlation can be analysed for the correlation.

6. Modeling on the extent of mixing versus time

Further work can also be done on the modeling of the extent of mixing versus time for a drop mixing between added new drops and existing old drops. With more detailed study, a population balance equation can also be determined. 


\section{CHAPTER 7}

\section{BIBLIOGRAPHY AND REFERENCES}

\subsection{Bibliography}

Billmeyer, F.W., "Textbook of Polymer Science", Wiley Interscience, New York (1983)

Hamielec, A.E and Tobita, H., "Polymerization Processes. Ullmann's Encyclopedia of Industrial Chemistry; VCH Publishers, Inc. New York, 1992, Vol A21, 305-428 (1992)

Odian, G., "Principles of Polymerization", John Wiley and Son, New York (1981)

Oldshue, J.Y., "Fluid Mixing Technology", McGraw-Hill Publication, New York (1983)

Robert J.H, "Introduction to Modern Colloid Science", Oxford Science Publications, Oxford, (1993) 
Rodriguez, F., "Principles of Polymer Systems", Hemisphere Publishing Corporation, New York (1982)

Rudin, A., "The Elements of Polymer Science and Engineering", Academic Press, New York, (1982)

\subsection{References}

Abraham, J., "Collision rates of small particles in a vigorously turbulent fluid", Chem. Eng. Sci., 30(11), p1371-1379(1975)

Adamson, A.W., "Physical Chemistry of Surfaces", $2^{\text {nd }}$ ed, Wiley, New York, p7881(1967)

Alvarez, J.M, Alvarez, J. and Hernandez M., "A population balance approach for the description of particle size distribution in suspension polymerization reactors", Chemical Eng. Sci, Vol. 49, No.1, p99-113 (1994).

Arai, K., Konno, M., Matunaga Y. and Saito, S., "Effect of dispersed-phase viscosity on the maximum stable drop size for breakup in turbulent flow", Journal of Chem. Eng. of Japan, Vol 10, No 4, p325-330 (1977)

Bajpai, R.K., Ramkrishna, D. and Prokop, A., "Coalescence redispersion for drop size distributions in agitated vessel," Chem. Eng. Sc., 31, p913-929 (1976)

Barber, A.D. and Hartland, "Effects of surface viscosity on the axisymmetric drainage of planar liquid films", J. Can. Journal of Chem. Eng. Vol 54, p278-284 (1976)

Borwankar R.P. , Chung S.I. and Wasan D.T., "Drop sizes in turbulent liquid-liquid dispersions containing polymeric suspension stabilizers I. The breakage mechanism". Journal of Polymer Sc., Vol 32, p5749-5762 (1986) 
Bouyatiotis, B.A. and Thornton, J.D., "Liquid-liquid extraction studies in stirred tank. Part 1: Droplet size and hold-up measurements in a seven-inch diameter baffled vessel", Institution of Chemical Engineers (London) Symposium Series, 26, p43-50 (1967)

Brooks, B.W., "Basic aspects and recent developments in suspension polymerisation", Makromol. Chem., Macromol. Symp., 35/36, p121-140 (1990)

Brown, D.E. and Pitt, K., "Drop size distribution of stirred non-coalescing liquidliquid system", Chem. Eng. Sci., 27, p577-583 (1972)

Calabrese R.V, Wang, C.Y.and Bryner N.P., "Drop breakup in turbulent stirred-tank contactors. Part III: Correlations for mean size and drop size distribution", AIChE Journal, Vol. 32, No. 4, p677-681 (1986b)

Calabrese R.V., Chang T.P.K. and Dang P.T., "Drop breakup in turbulent stirred-tank contactors. Part I: Effect of dispersed-phase viscosity", AIChE Journal, Vol 32, No. 4, p657-666 (1986a)

Chatzi, E.G. and Lee J.M., "Analysis of Interactions for liquid-liquid dispersions in agitated vessels", Ind. Eng. Chem. Res., 26, p2263 (1987)

Chatzi E.G, Costas J.B and Costas K., "On-line monitoring of drops size distributions in agitated vessels. 2. Effect of Stabilizer Concentration”, Ind. Eng. Chem. Res., 30, p1307-1313, (1991)

Chatzi E.G. and Kiparissides C., "Steady state drop-size distribution in high holdup fraction dispersions systems", AIChE J., Vol 41, No 7, p1640-1652. (1995)

Chen, H.T. and Middleman, S., "Drop size distribution in agitated liquid-liquid systems", AIChe J., Vol 13, p989-995 (1967)

Chester, A.K. (1991)," The modelling of coalescence process in fluid-fluid dispersion: a review of current understanding”, Trans Intn. Chem. Eng, 69, p259-270 (1991) 
Church, J.M. and Shinnar, R., "Stabilising liquid-liquid dispersions by agitation", Ind. Eng. Chem., 53, No. 6, p479-484 (1961)

Coulaloglou, C.A. and Tavlarides L.L, "Description of interaction processes in agitated liquid-liquid dispersions", Chem. Eng. Sci., Vol. 32, p289-1297 (1977)

Coulaloglou, C.A. and Tavlarides L.L, "Drop size distributions and coalescence frequencies of liquid-liquid dispersions in flow vessels", AIChE J., Vol. 32, p289-297 (1976)

Das, P.K., Kumar, R. and Ramkrishna, D., "Coalescence of drops in stirred dispersions. A white noise model for coalescence", Chem. Eng. Sci, 42, No.2, p213220. (1987).

Doulah, M.S.,"An effect of hold-up on drop sizes in liquid-liquid dispersions, Ind. Engng Chem. Fundamental 14, p137-138 (1975)

Hahn P.S. and Slattery, J.C., "Effects of surface viscosities on the stability of draining plane parallel liquid film as a small bubble approaches a liquid-gas interface. AIChE J 31 p959-956, (1985)

Hartland, S., "The coalescence of a liquid-liquid interface, Part 5. The effect of surface active agent", Trans. Inst. Chem. Eng. (London), 46, T275 (1968).

Hinze, J.O., "Fundamentals of the hydrodynamic mechanism of splitting in dispersions processes", AIChE J., Vol.1, No. 3, p289-285 (1955)

Hong, P.O. and Lee, J.M., "Unsteady state liquid-liquid dispersions in agitated vessels", Ind. \& Eng Chem Process Des Dev., 22, p130-135 (1983)

Hong, P.O. and Lee, J.M., "Changes of the average drop sizes during the initial period of liquid-liquid dispersions in agitated vessels", Ind. \& Eng Chem Process Des Dev, 24, p868-872 (1985) 
Howarth, W.J, "Coalescence of drops in a turbulent flow field", Chem. Eng. Sci., Vol 19, p33-38 (1964)

Hulburt, H.M. and Katz, S., "Some problems in particle technology a statiscal mechanical formulation", Chem. Eng. Sci, p16, 555-574 (1964)

Ivanov, I.B. and Traykov, T.T., "Hydrodynamics of thin liquid films: rate of thinningof emulsion films from the pure liquids", Int. J. Multiphase Flow, Vol. 2, p397-410 (1976).

Ivanov, I.B., Dimitrov, D.S., Somasundaran, P., and Jain, R.K., "Thinning of films with deformable surfaces: diffusion-controlled surfactant transfer"” Chem. Eng. Sci. $40, \mathrm{p} 137-150(1985)$

Kolmogorov, A.N., "The local structure of turbulence in incompressible viscous fluid for very large Reynolds numbers", C.R. Acad. Sci, U.S.S.R., 30, p301 (1941)

Komasawa, I., Sasakura, T. and Otake, T., "Behavior of reacting and coalesing dispersed phase in a stirred tank reactor", J. Chem. Eng. Japan, 16, 208 (1969)

Konno, M., Arai, K. and Shozaburo, S., "The effect of stabilizer on coalescence of dispersed drops in suspension polymerization of styrene", Journal of Chem. Eng. Of Japan, Vol. 15, No.2, p131-135 (1982).

Konno. M, Kosaka, N. and Saito, S., "Correlation of transient drop sizes in break-up process in liquid-liquid agitation", J. Chem. Eng. Japan, Vol. 26, No.1, p37-40 (1993)

Koshy, A. Das, T.R. and Kumar, R. 1988, "Effect of surfactants on drop breakage in turbulent liquid dispersions", 43, p649-654.

Kumar, S., Kumar, R. and Gandhi, K.S., "A new model for coalescence efficiency of drops in stirred dispersions", Chem. Eng. Sci., Vol. 48, No.11, p2025-2038 (1993) 
Kuriyama M., Ono M., Tokonai H. and Konno H., "Correlation of transient sizes of highly viscous drops in dispersion process in liquid-liquid agitation", Trans IchemE, Vol. 74, Part A. p431-437 (1996).

Lagisetty, J.S., Das, P.K. Kumar,Rand Gandhi, K.S., "Breakage of viscous and nonnewtonian drops in stirred dispersions. Chem. Eng. Sci 42, p2429-2434 (1986)

Laso M., Steiner L. and Hartland S, "Dynamic Simulation of agitated liquid-liquid dispersions-II. Experimental determination of breakage and coalescence rates in a stirred tank" Chem. Eng. Sc., Vol 42, No 10, p2437-2445 (1987)

Lee, J.C. and Tasakorn, P., "Characteristics of agitated tanks in relation to suspension polymerization. $3^{\text {rd }}$ European Conference on Mixing", University of York, England, 4-6 April 1979.

Leng, D.E. and Quarderer, G.J., "Drop dispersion in suspension polymerization", Chem. Eng. Commun. 14, 177-201 (1982)

Lui, S. and Li, D. "Drop coalescence in turbulent dispersions", Chem. Eng. Sci., 54, p5667-5675 (1999)

Madden, A.J. and Daramell, G.L., Coalescence frequencies in agitated liquid-liquid dispersion, AIChE Journal, 8, p233-239 (1962)

Maggioris, D., Goulas, A., Alexopouluos, A.H., Chatzi, E.G. and Kipparissides, C., "Prediction of particle size distribution in suspension polymerization reactors: effect of turbulence nonhomogeneity", Chem. Eng. Sci, 55, p4611-4627 (2000)

Mendizabal, E., Casterllanos-Ortega, J.R. and Puig, JE. "A method for selecting polyvinyl alcohol as stabiliser in suspension polymerization", Colloid Surf., 63, p209217 (1992).

Miller, R.S., Ralf, J.L., Curl, R.L. and Towell, G.D., "Dispersed phase mixing :2Measurement in organic dispersed systems", AIChE, 9, No.2, 196-202 (1963) 
Narsimhan, G., Gupta, J.P. and Ramakrishna, D., "A model for transitional breakage probability of droplets in agitated lean liquid-liquid dispersion", Chem. Eng Sci, 34, p257-265 (1979)

Nienow, A.W. and Miles, D., "Impeller power number is close vessels", Ind. Eng. Chem. Process Des. Dev, 10, p41 (1971)

Nielson, L.E., Mechanical Properties of Polymers and Composites, Dekker, New York (1974)

Ottewill, R.H., in "Non-ionic surfactants", Schick, M.J. edition, p645, Edwards Arnold, London (1967).

Park, J.Y. and Blair, L.M., "The effect of coalescence on drop size distribution in an agitated liquid-liquid dispersion", Chem. Eng. Sci, 30, p1057-1064 (1975)

Ramkrishna, D., "Drop break-up in agitated liquid-liquid dispersion", Chem. Eng. Sci, 29, p987-992 (1974).

Ray, W.H., "Modelling of addition polymerization processes. Free radical, ionic, group transfer and Zeigler-Natta Kinetics, Can. J. Chem. Eng., 69, p626-629 (1991)

Rosen, M.J., "Emulsification by surfactants", in "Surfactants and interfacial phenomena", Second ed., John Wiley \& Son, New York (1989)

Ross, S.L. , Verhoff, F.H. and Curl, R.L., "Droplet breakage and coalescence in an agitated dispersion 2. Measurement and interpretation of mixing of experiments', Ind. Eng. Chem. Fundam., 17, 2, p101-108 (1978)

Rushton, J.H., Costich, E.W. and Everett, H.J., "Power characteristics of mixing impellers", Chem. Eng. Prog., 46, p395 (1950)

Shinnar, R. and Church, J.M., "Statistical theories of turbulence in predicting particle size in agitated dispersions", Ind. Eng. Chem., 52, p253-256 (1960) 
Shinnar, R., "On the behaviour of liquid dispersions in mixing vessels", J. Fluid Mech., 10. p259-275 (1961)

Skelland, A.H. P. and Kanel, J. S., "Minimum impeller speeds for complete dispersion of non-newtonian liquid-liquid system in baffled vessel"' Ind. Eng. Chem, Res, 29, p1300-1306 (1990).

Sovova, H., "Breakage and coalescence of drops in a batch stirred vessel - I. Comparison of Continuous and Discrete Models", Chem. Eng. Sci., 36, n1, p163-171 (1981)

Sovova, H., "Breakage and coalescence of drops in a batch stirred vessel - II. Comparison of model and experiments", Chem. Eng. Sci., 36, n9, p1567-1573 (1981)

Sprow, F.B, "Distribution of drop sizes produced in turbulent liquid-liquid dispersions”, Chem. Eng. Sci.,22, p435-442 (1967)

Stamatoudis, M. and Tavlarides, L.L., "Effect of continuous phase viscosity on the drops sizes of liquid-liquid dispersions in agitated vessels", Ind. Eng. Chem. Process Des. Dev., 24, p175-1181 (1985).

Stephenson, R.C. and Smallwood, P.V., "Suspension and mass polymerisation", Encyclopedia of polymer science and engineering, Second Edition, Vol. 17 and Supplement, John Wiley and Son, (1989)

Tobin, T., Muralidhar R., Write H. and Ramkrishna, D., "Determination of coalescence frequencies in liquid-liquid dispersions: Effect of drop size dependence," Chem. Eng. Sci, 45, p3491 (1990).

Tsouris, S. and Tavlarides, L.L., "Breakage and Colescence Models for Drops in Turbulent Dispersions", AIChE Journal, Vol.40, No.3, p395-406 (1994) 
Valentas K.J.and Amundson N.R., "Breakage and Coalescence in dispersed phase systems", Ind. Eng. Chem. Fundam., 5(4), p533-542 (1966)

Valentas, K.H., Bilous, O. and Amundson, N.R., "Analysis of breakage in dispersed phase systems", Ind. Eng. Chem. Fundam., 5(2), p271-279 (1966)

Verhoff, F.H., Ross, S.L. and Curl, R.L., "Breakage and coalescence processes in an agitated dispersion: Experimental system and data reduction", Ind. Eng. Chem. Fund., 16, 3, p371-377 (1977)

Vermeulen, T., Williams, G.M. and Langlois, G.E. , "Interfacial area in liquid-liquid and gas -liquid agitation”, Chemical Eng. Progress, 51, 85F-94F (1955)

Vivaldo-Lima, E., Wood, P.E. and Hamielec, A. E., "An updated Review on Suspension Polymerization", Ind. Eng. Chem . Res., 36, p939-965 (1997)

Walstra P., "Principles of emulsion formation", Chem. Eng. Sci., Vol 46, p333-349 (1993)

Wang, C.Y.and Calabrese R.V., "Drop breakup in turbulent stirred-tank contactors. Part II: Relative Influence of viscosity and interfacial tension”, AIChE Journal, Vol. 32, No. 4, p667-676 (1986)

Wright, H. and Ramkrishna, D., "Factors affecting coalescence frequency of droplets in a stirred liquid-liquid dispersions", AIChE J., Vol. 40, No. 5, p767-776 (1994)

Yuan, H.G.; Kalfas, G and Ray, W.H., "Suspension Polymerisation", JMS-REV. Macrool. Chem. Phys., C31(2\&3), p215-299 (1991)

Zerfa, M., "Vinyl Chloride drop behavior in suspension polymerisation reactors", $\mathrm{PhD}$ Thesis, Loughborough University of Technology (1994)

Zerfa M. and Brooks B.W., "Vinyl Chloride Dispersion with relation to suspension polymerization", Chem. Eng. Sci., Vol. 51, No. 14, p3591-3611 (1996a) 
Zerfa M. and Brooks B.W., "Drop Coalescence Processes in Suspension Polymerization of Vinyl Chloride," J. Applied Polymer Sc., Vol. 60, p2077-2086 (1996b). 


\section{APPENDIX A}

\section{Viscosity Measurement}

\section{Theory}

Viscosity of the dispersed phase solution was determined by the glass capillary method. This method is widely used for low viscosity polymer solutions. The method measures the time it takes a volume of the polymer solution to flow through a thin capillary. This value is then compared to the time for a known viscosity and density liquid (standard). It turns out that the flow time for either is proportional to the viscosity, and inversely proportional to the density.

$$
\begin{aligned}
& t_{\text {unknown }}=\frac{\mu_{\text {unknown }}}{\rho_{\text {unknown }}} \\
& t_{\text {known }}=\frac{\mu_{\text {known }}}{\rho_{\text {known }}}
\end{aligned}
$$

or can be written as

$$
\frac{\mu_{\text {un known }}}{\mu_{\text {known }}}=\frac{t_{\text {unknown }} \rho_{\text {unknown }}}{t_{\text {known }} \rho_{\text {known }}}
$$

Therefore viscosity of the measured liquid can be determined.

\section{Experimental Procedure}

The measurement using the glass capillary viscometer was done in constant temperature bath (water reservoir). The water was allowed to attain thermal equilibrium before the measurement was made. A rubber suction tube was used to draw the liquid into the upper level. The time was measured for the level of the liquid to pass the markings. Several glass viscometer were used to measure the viscosity of different concentration of the polymer solution. The higher the viscosity, the bigger 
size of glass viscometer needed. At least five measurements were done for each concentration of polymer solution, then an average was taken. 


\section{APPENDIX B}

\section{Surface Tension Measurement}

\section{Theory}

The surface tension measurements were made using a $\mathrm{Du}$ Nouy ring tensiometer. This method is one of the most widely used and effective methods of determining surface tension between interface of two liquids. The method is to measure the force required to pull a ring from the interface.

Figure B.1 shows the schematic diagram of the Du Nouy ring tensiometer. The ring is hanging on a microbalance. The force is measured directly using the microbalance to determine the apparent weight of a ring of platinum as it is pulled through the liquid-liquid interface.

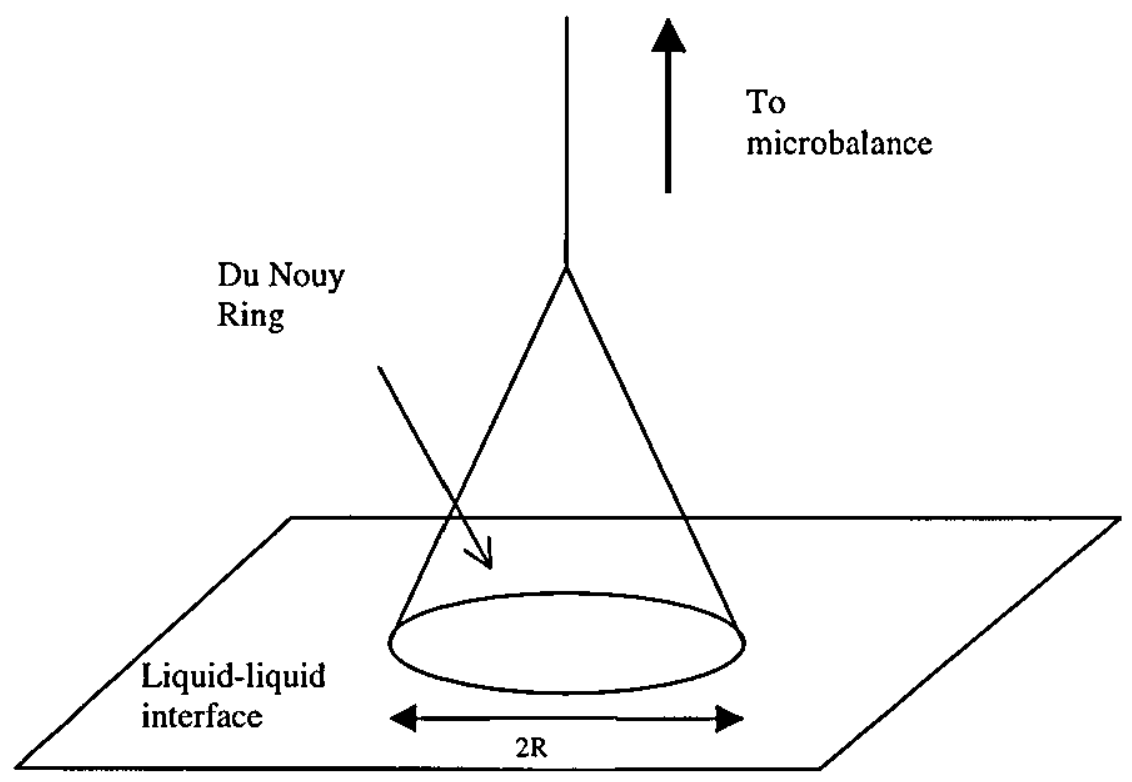

Figure B.1: Schematic diagram of the Du Nouy ring tensiometer 
The surface tension force acts around the perimeter of the ring which is being withdrawn so the foce is easily calculated as

$$
\text { Force }(\mathrm{F})=\text { Surface tension }(\sigma) \times \text { perimeter of the ring }
$$

The meniscus forms on both sides of the ring so the force is given by

$$
\mathrm{F}=4 \pi \mathrm{r} \sigma=\mathrm{mg}
$$

where $m$ is the apparent mass as the ring comes through the surface.

\section{Experimental Method}

In order to simulate the liquid-liquid interface in the dispersion experiments, total surface area of the drops were determined. By assuming that all surfactant molecules were attached between the interface, the amount of surfactant/surface area was determined. These values are used in the measuring the surface tension using the Du Nuoy method. By dissolving the specific amount of surfactant in the water and using a specific concentration of polystyrene solution, the surface tension between the two liquids was determined. The measurement was repeated for at least five times and an average value was taken. 


\section{APPENDIX C}

EXPERIMENTAL DATA 
Table C4.1: Batch mixing of new toluene with the existing dispersed phase using 0.02 wt \% PVA

\begin{tabular}{|c|c|c|}
\hline & \multicolumn{2}{|c|}{ Extent of mixing for different dispersed phase } \\
\hline Time (minute) & Toluene & $10 \mathrm{~g}$ PS/ 50ml toluene \\
\hline 0 & 0.500 & 0.500 \\
\hline 5 & 0.650 & 0.635 \\
\hline 15 & 0.717 & 0.704 \\
\hline 30 & 0.816 & 0.776 \\
\hline 45 & 0.843 & 0.775 \\
\hline 60 & 0.932 & 0.819 \\
\hline 90 & 0.967 & 0.852 \\
\hline 120 & 0.976 & 0.934 \\
\hline
\end{tabular}

Table C4.2: Extent of mixing between two dispersion of different surfactant

\begin{tabular}{|c|c|c|}
\hline \multirow{2}{*}{$\begin{array}{l}\text { Time } \\
\text { (minutes) }\end{array}$} & \multicolumn{2}{|l|}{ Extent of mixing } \\
\cline { 2 - 3 } & Exp1 & Exp2 \\
\hline 0 & 0.500 & 0.500 \\
\hline 5 & 0.806 & 0.556 \\
\hline 15 & 0.902 & 0.640 \\
\hline 30 & 0.974 & 0.761 \\
\hline 45 & 1.000 & 0.814 \\
\hline 60 & 1.000 & 0.839 \\
\hline 90 & 1.000 & 0.888 \\
\hline 120 & 1.000 & 0.919 \\
\hline
\end{tabular}

Exp1 - Dispersion of toluene without stabiliser and dispersion of toluene with $0.02 \%$ PVA

Exp2 - Dispersion of toluene without stabiliser and dispersion of toluene with $0.02 \%$ Tween 20 
Table C4.3: Drop size distribution for mixing of dispersion for two different conditions at 120 minutes

\begin{tabular}{|c|c|c|c|c|}
\cline { 2 - 5 } \multicolumn{1}{c|}{} & \multicolumn{3}{c|}{ Frequency at different condition } \\
\cline { 2 - 5 } \multicolumn{1}{c|}{} & \multicolumn{2}{c|}{ Exp1 } & \multicolumn{2}{c|}{ Exp2 } \\
\hline Bin & coloured & uncoloured & coloured & uncoloured \\
\hline 10 & 0 & none & 0 & 0 \\
\hline 20 & 6 & & 0 & 0 \\
\hline 30 & 54 & & 9 & 0 \\
\hline 40 & 55 & & 34 & 11 \\
\hline 50 & 77 & & 60 & 13 \\
\hline 60 & 42 & & 65 & 15 \\
\hline 70 & 31 & & 51 & 8 \\
\hline 80 & 26 & & 48 & 4 \\
\hline 90 & 7 & & 45 & 2 \\
\hline 100 & 6 & & 17 & 0 \\
\hline 120 & 0 & & 11 & 0 \\
\hline 130 & 0 & & 2 & 0 \\
\hline 140 & 0 & & 0 & 0 \\
\hline 150 & 0 & & 0 & 0 \\
\hline More & 0 & & 0 & 0 \\
\hline
\end{tabular}

Exp1 - Dispersion of toluene without stabiliser and dispersion of toluene with $0.02 \%$ PVA

Exp2 - Dispersion of toluene without stabiliser and dispersion of toluene with $0.02 \%$ Tween 20

Table C4.4: Extent of mixing for two different conditions

\begin{tabular}{|c|c|c|}
\hline \multirow{2}{*}{ Time(min) } & \multicolumn{2}{|c|}{ Extent of mixing } \\
\cline { 2 - 3 } & Exp1 & Exp2 \\
\hline 0 & 0.500 & 0.500 \\
\hline 5 & 0.528 & 0.556 \\
\hline 15 & 0.567 & 0.640 \\
\hline 30 & 0.594 & 0.761 \\
\hline 45 & 0.653 & 0.814 \\
\hline 60 & 0.740 & 0.839 \\
\hline 90 & 0.771 & 0.885 \\
\hline 120 & 0.861 & 0.919 \\
\hline
\end{tabular}

Exp1 - Dispersion of toluene with $0.02 \%$ Tween 20 and

dispersion of toluene with $0.02 \%$ Tween 20

Exp2 - Dispersion of toluene without stabiliser and dispersion of toluene with $0.02 \%$ Tween 20 
Table C4.5: Mean drop size as function of stirring time for different polystyrene solution

\begin{tabular}{|c|c|c|c|}
\hline & \multicolumn{3}{|c|}{ Average Drop Size $(\mu \mathrm{m})$} \\
\hline Time (min) & 5 wt \% PS & 10 w\% PS & 15 wt\% PS \\
\hline 5 & 32.57 & 70.56 & 87.39 \\
\hline 15 & 26.22 & 34.77 & 45.68 \\
\hline 30 & 22.54 & 24.05 & 33.72 \\
\hline 45 & 23.77 & 25.73 & 34.51 \\
\hline 60 & 22.96 & 23.57 & 30.68 \\
\hline 90 & 22.13 & 25.68 & 30.32 \\
\hline 120 & 20.46 & 25.27 & 31.92 \\
\hline 150 & 20.15 & 25.25 & 31.96 \\
\hline
\end{tabular}

Table C4.6: Drop size distribution of different polystyrene solution at 120 minutes

\begin{tabular}{|c|c|c|c|}
\hline & \multicolumn{4}{|c|}{ Frequency at different concentration } \\
\hline Bin & $5 \mathrm{wt} \%$ & $10 \mathrm{wt} \%$ & $15 \mathrm{wt} \%$ \\
\hline 10 & 61 & 39 & 15 \\
\hline 20 & 81 & 63 & 46 \\
\hline 30 & 172 & 147 & 82 \\
\hline 40 & 37 & 75 & 66 \\
\hline 50 & 4 & 30 & 52 \\
\hline 60 & 0 & 8 & 22 \\
\hline 70 & 0 & 4 & 13 \\
\hline 80 & 0 & 1 & 3 \\
\hline 90 & 0 & 0 & 0 \\
\hline 100 & 0 & 0 & 0 \\
\hline More & 0 & 0 & 0 \\
\hline
\end{tabular}


Table C4.8: Extent of mixing for batch mixing of new styrene with different viscosities of dispersed phase at $500 \mathrm{rpm}$

\begin{tabular}{|c|c|c|c|c|}
\hline Time & \multicolumn{5}{|l|}{ Extent of mixing } \\
\hline (minute) & 5 wt \% & 10 wt \% & 15 wt \% & 20 wt \% \\
\hline 0 & 0.200 & 0.200 & 0.200 & 0.200 \\
\hline 5 & 0.667 & 0.357 & 0.515 & 0.686 \\
\hline 15 & 0.832 & 0.581 & 0.607 & 0.862 \\
\hline 30 & 0.879 & 0.635 & 0.851 & 0.916 \\
\hline 45 & 0.901 & 0.679 & 0.853 & 0.942 \\
\hline 60 & 0.925 & 0.776 & 0.895 & 0.971 \\
\hline 90 & 0.973 & 0.781 & 0.967 & 0.972 \\
\hline 120 & 0.967 & 0.837 & 0.988 & 0.997 \\
\hline 150 & 0.966 & 0.837 & 0.993 & 1.000 \\
\hline 180 & 0.960 & 0.939 & 1.000 & 1.000 \\
\hline
\end{tabular}

Figure C4.9: Drop size distribution for batch mixing at 180 minutes (500 rpm)

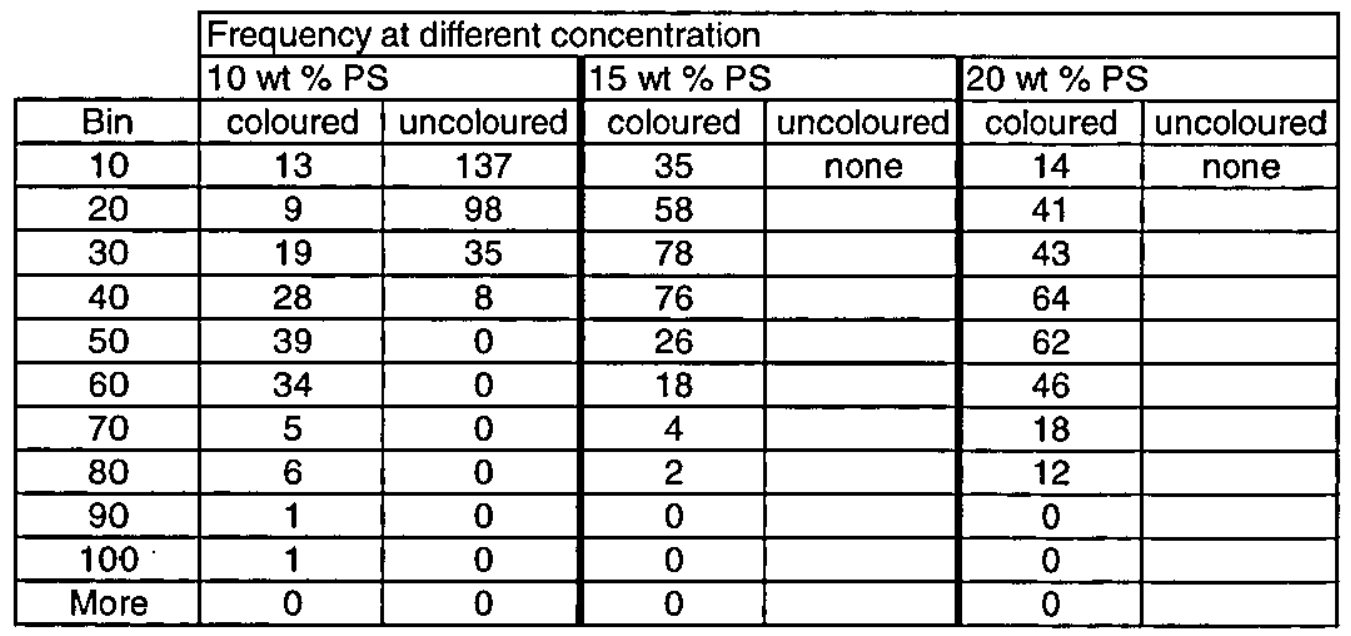


Table C4.10 : Drop size distribution for batch mixing of styrene with $20 \mathrm{wt} \%$ dispersed phase

\begin{tabular}{|c|c|c|c|c|c|c|}
\hline \multirow[b]{3}{*}{ Bin } & \multicolumn{6}{|c|}{ Frequency at different time } \\
\hline & \multicolumn{2}{|c|}{5 minutes } & \multicolumn{2}{|l|}{15 minutes } & \multicolumn{2}{|l|}{30 minutes } \\
\hline & coloured & uncoloured & coloured & uncoloured & coloured & uncoloured \\
\hline 10 & 0 & 39 & 1 & 17 & 1 & 51 \\
\hline 20 & 2 & 79 & 5 & 45 & 12 & 75 \\
\hline 30 & 8 & 51 & 11 & 34 & 13 & 52 \\
\hline 40 & 19 & 48 & 49 & 16 & 50 & 12 \\
\hline 50 & 23 & 21 & 53 & $\begin{array}{ll}7 & \\
\end{array}$ & 45 & 2 \\
\hline 60 & 17 & 5 & 29 & 6 & 22 & 0 \\
\hline 70 & 17 & 3 & 18 & 2 & 12 & 0 \\
\hline 80 & 8 & 3 & 7 & 0 & 12 & 0 \\
\hline 90 & 4 & 1 & 3 & 0 & 4 & 1 \\
\hline 100 & 2 & 1 & 1 & 0 & 1 & 0 \\
\hline 110 & 1 & 0 & 0 & 0 & 1 & 0 \\
\hline 120 & 0 & 0 & 0 & 0 & 0 & 0 \\
\hline \multirow[t]{3}{*}{ More } & 0 & 0 & 0 & 0 & 0 & 0 \\
\hline & \multicolumn{6}{|c|}{ Frequency at different time } \\
\hline & 45 minutes & & \multicolumn{2}{|l|}{60 minutes } & \multicolumn{2}{|l|}{90 minutes } \\
\hline Bin & coloured & uncoloured & coloured & uncoloured & coloured & uncoloured \\
\hline 10 & 0 & 60 & 2 & 62 & 6 & 71 \\
\hline 20 & 11 & 46 & 1 & 45 & 25 & 76 \\
\hline 30 & 23 & 20 & 19 & 23 & 56 & 12 \\
\hline 40 & 43 & 14 & 33 & 6 & 59 & 7 \\
\hline 50 & 34 & 4 & $\overline{43}$ & $\overline{1}$ & 38 & 0 \\
\hline 60 & 15 & 0 & 27 & 0 & 24 & 0 \\
\hline 70 & 11 & 0 & 22 & 0 & 13 & 0 \\
\hline 80 & $\begin{array}{c}8 \\
8\end{array}$ & 0 & 16 & 0 & 9 & 0 \\
\hline 90 & 10 & 0 & 8 & 0 & 3 & 0 \\
\hline 100 & 1 & 0 & 0 & 0 & 0 & 0 \\
\hline 110 & 0 & 0 & 0 & 0 & 0 & 0 \\
\hline 120 & 0 & 0 & 0 & 0 & 0 & 0 \\
\hline \multirow[t]{3}{*}{ More } & 0 & 0 & 0 & 0 & 0 & 0 \\
\hline & \multicolumn{6}{|c|}{ Frequency at different time } \\
\hline & \multicolumn{2}{|c|}{120 minutes } & \multicolumn{2}{|l|}{150 minutes } & \multicolumn{2}{|l|}{180 minutes } \\
\hline Bin & coloured & uncoloured & coloured & uncoloured & coloured & uncoloured \\
\hline 10 & 35 & 32 & 31 & 24 & 14 & none \\
\hline 20 & 54 & 9 & 73 & 10 & 41 & \\
\hline 30 & 62 & 2 & 61 & 0 & 43 & \\
\hline 40 & 66 & 0 & 41 & 0 & 64 & \\
\hline 50 & 37 & 0 & 40 & 0 & 62 & \\
\hline 60 & 15 & 0 & 12 & 0 & 46 & \\
\hline 70 & 15 & 0 & 6 & 0 & 18 & \\
\hline 80 & 1 & 0 & 3 & 0 & 12 & \\
\hline 90 & 1 & 0 & 0 & 0 & 0 & \\
\hline 100 & 0 & 0 & 0 & 0 & 0 & \\
\hline 110 & 0 & 0 & 0 & 0 & 0 & \\
\hline 120 & 0 & 0 & 0 & 0 & 0 & \\
\hline More & 0 & 0 & 0 & 0 & 0 & \\
\hline
\end{tabular}


Table C4.11: Mean drop size for coloured and uncoloured drops at $15 \mathrm{wt} \%$ PS and $20 \mathrm{wt} \%$ PS dispersed phase at $500 \mathrm{rpm}$

15 wt \% PS

\begin{tabular}{|c|c|c|}
\hline & \multicolumn{2}{|c|}{ Mean drop size $(\mu \mathrm{m})$} \\
\hline Stirring time & coloured & uncoloured \\
\hline 5 & 36.93 & 19.70 \\
\hline 15 & 37.64 & 22.02 \\
\hline 30 & 39.31 & 17.70 \\
\hline 45 & 36.43 & 15.38 \\
\hline 60 & 37.84 & 16.19 \\
\hline 90 & 42.27 & 11.70 \\
\hline 120 & 29.58 & 16.77 \\
\hline 150 & 28.50 & 12.42 \\
\hline 180 & 26.73 & \\
\hline \multicolumn{3}{|c}{} \\
\hline
\end{tabular}

$20 \mathrm{wt} \%$ PS

\begin{tabular}{|c|c|c|}
\hline & \multicolumn{2}{|c|}{ Mean drop size $(\mu \mathrm{m})$} \\
\hline Stirring time & coloured & uncoloured \\
\hline 5 & 51.11 & 23.52 \\
\hline 15 & 45.69 & 22.93 \\
\hline 30 & 44.74 & 16.42 \\
\hline 45 & 43.96 & 14.84 \\
\hline 60 & 48.49 & 13.20 \\
\hline 90 & 36.86 & 11.55 \\
\hline 120 & 29.02 & 8.33 \\
\hline 150 & 26.67 & 8.27 \\
\hline 180 & 37.24 & \\
\hline
\end{tabular}

Table C4.12: Extent of mixing for batch mixing of new styrene with different viscosities of dispersed phase

\begin{tabular}{|c|c|c|}
\hline Time & \multicolumn{2}{|l|}{ Extent of mixing } \\
\hline (time) & $15 \%$ PS & $20 \%$ PS \\
\hline 0 & 0.200 & 0.200 \\
\hline 5 & 0.649 & 0.811 \\
\hline 15 & 0.904 & 0.969 \\
\hline 30 & 0.986 & 0.991 \\
\hline 45 & 0.981 & 0.996 \\
\hline 60 & 0.989 & 0.996 \\
\hline 90 & 0.995 & 0.996 \\
\hline 120 & 0.991 & 0.996 \\
\hline 150 & 1.000 & 0.996 \\
\hline 180 & 1.000 & 0.999 \\
\hline
\end{tabular}


Table C4.13: Drop size distribution for batch mixing of styrene with 15 wt \% PS dispersed phase at $650 \mathrm{rpm}$

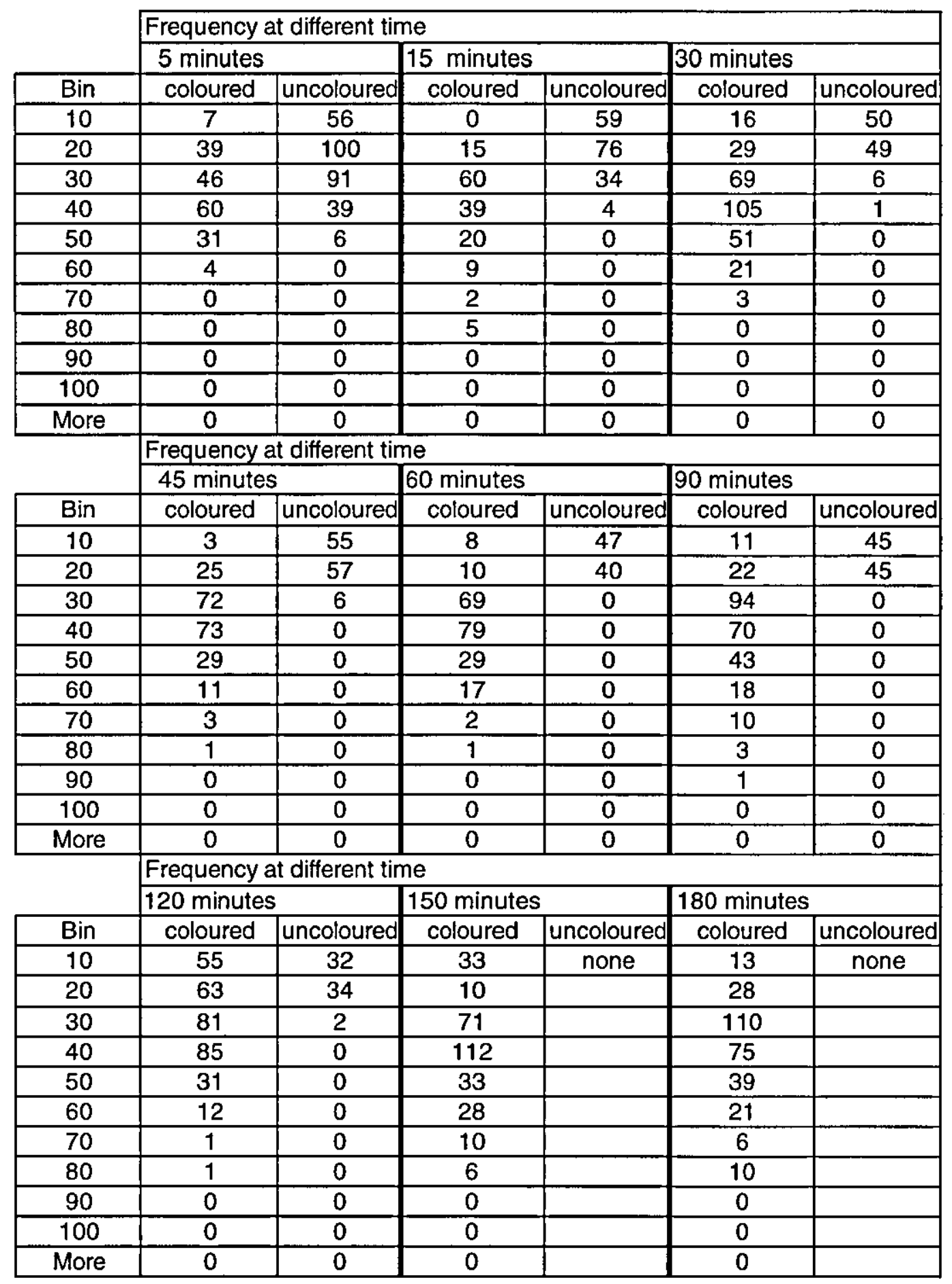


Table C4.14: Extent of mixing for batch mixing of new styrene with different viscosities of dispersed phase at $350 \mathrm{rpm}$

\begin{tabular}{|c|c|c|c|}
\hline Time & \multicolumn{4}{|l|}{ Extent of mixing } \\
\hline (time) & $5 w t \%$ PS & $10 w t \% P S$ & $15 w t \%$ PS \\
\hline 0 & 0.200 & 0.200 & 0.200 \\
\hline 5 & 0.389 & 0.297 & 0.257 \\
\hline 15 & 0.431 & 0.306 & 0.284 \\
\hline 30 & 0.463 & 0.387 & 0.328 \\
\hline 45 & 0.455 & 0.443 & 0.406 \\
\hline 60 & 0.496 & 0.440 & 0.392 \\
\hline 90 & 0.489 & 0.452 & 0.428 \\
\hline 120 & 0.511 & 0.491 & 0.412 \\
\hline 150 & 0.649 & 0.582 & 0.419 \\
\hline 180 & 0.664 & 0.592 & 0.495 \\
\hline
\end{tabular}


Table C4.15: Drop size distribution for batch mixing of styrene with 15 wt \% PS dispersed phase at $350 \mathrm{rpm}$

\begin{tabular}{|c|c|c|c|c|c|c|}
\hline & \multicolumn{6}{|c|}{ Frequency at different time } \\
\hline & \multicolumn{2}{|l|}{5 minutes } & \multicolumn{2}{|l|}{15 minutes } & \multicolumn{2}{|l|}{30 minutes } \\
\hline Bin & coloured & uncoloured & coloured & uncoloured & coloured & uncoloured \\
\hline 20 & 3 & 16 & 10 & 50 & 11 & 30 \\
\hline 40 & 33 & 21 & 43 & 19 & 62 & 29 \\
\hline 60 & 74 & 18 & 38 & 19 & 96 & 23 \\
\hline 80 & 87 & 13 & 88 & 13 & 105 & 29 \\
\hline 100 & 60 & 16 & 36 & 19 & 31 & 15 \\
\hline 120 & 26 & 7 & 8 & 14 & 4 & 19 \\
\hline 140 & 9 & 2 & 2 & 14 & 5 & 15 \\
\hline 160 & 3 & 7 & 1 & 31 & 1 & 12 \\
\hline 180 & 1 & 9 & 1 & 8 & 0 & 17 \\
\hline 200 & 1 & 3 & 1 & 5 & 0 & 4 \\
\hline 220 & 0 & 1 & 1 & 1 & 0 & 0 \\
\hline 240 & 0 & 2 & 0 & 1 & 1 & 0 \\
\hline \multirow[t]{3}{*}{ More } & 0 & 0 & 0 & 0 & 0 & 0 \\
\hline & \multicolumn{6}{|c|}{ Frequency at different time } \\
\hline & \multicolumn{2}{|c|}{45 minutes } & \multicolumn{2}{|l|}{60 minutes } & \multicolumn{2}{|l|}{90 minutes } \\
\hline Bin & coloured & uncoloured & coloured & uncoloured & coloured & uncoloured \\
\hline 20 & 34 & 49 & 16 & 16 & 32 & 36 \\
\hline 40 & 75 & $\overline{55}$ & 93 & 21 & 100 & 28 \\
\hline 60 & 96 & 21 & 120 & 18 & 117 & 20 \\
\hline 80 & 100 & 28 & 117 & 13 & 109 & 17 \\
\hline 100 & 27 & 16 & 28 & 16 & 18 & 8 \\
\hline 120 & 1 & 10 & 6 & 7 & 2 & 6 \\
\hline 140 & 1 & 10 & 4 & 2 & 4 & 13 \\
\hline 160 & 0 & 8 & 0 & 7 & 0 & 8 \\
\hline 180 & 0 & 4 & 0 & 9 & 2 & 4 \\
\hline 200 & 0 & 1 & 0 & 3 & 1 & 2 \\
\hline 220 & 0 & 0 & 0 & 1 & 0 & 1 \\
\hline \multirow{4}{*}{$\begin{array}{l}240 \\
\text { More }\end{array}$} & 0 & 1 & 0 & 2 & 0 & 0 \\
\hline & 0 & 0 & 0 & 0 & 0 & 0 \\
\hline & \multicolumn{6}{|c|}{ Frequency at different time } \\
\hline & \multicolumn{2}{|c|}{120 minutes } & \multicolumn{2}{|c|}{150 minutes } & \multicolumn{2}{|c|}{180 minutes } \\
\hline Bin & coloured & uncoloured & coloured & uncoloured & coloured & uncoloured \\
\hline 20 & 20 & 11 & 13 & 13 & 31 & 7 \\
\hline 40 & 61 & 10 & 77 & 20 & 91 & 8 \\
\hline 60 & 131 & 14 & 121 & 16 & 105 & 6 \\
\hline 80 & 93 & 13 & 90 & 13 & 62 & 10 \\
\hline 100 & 15 & 10 & 23 & 8 & 22 & 5 \\
\hline 120 & 12 & 13 & 4 & 7 & 7 & 5 \\
\hline 140 & 6 & 11 & 6 & 6 & 5 & 9 \\
\hline 160 & 0 & 8 & 1 & 5 & 2 & 9 \\
\hline 180 & 2 & 7 & 0 & 12 & 0 & 6 \\
\hline 200 & 0 & 4 & 0 & 0 & 0 & 1 \\
\hline 220 & 0 & 0 & 0 & 0 & 0 & 1 \\
\hline 240 & 0 & 0 & 0 & 0 & 1 & 0 \\
\hline More & 0 & 0 & 0 & 0 & 0 & \\
\hline
\end{tabular}


Table C4.16: Extent of mixing for $15 \mathrm{wt} \%$ PS dispersed phase running at different stirrer speed

\begin{tabular}{|c|c|c|c|}
\hline $\begin{array}{l}\text { Stirring } \\
\text { time }\end{array}$ & \multicolumn{3}{|l|}{$\begin{array}{l}\text { Extent of mixing at different } \\
\text { stirring speed }\end{array}$} \\
\hline (minutes) & $350 \mathrm{rpm}$ & $500 \mathrm{rpm}$ & $650 \mathrm{rpm}$ \\
\hline 0 & 0.200 & 0.200 & 0.200 \\
\hline 5 & 0.257 & 0.514 & 0.649 \\
\hline 15 & 0.284 & 0.607 & 0.904 \\
\hline 30 & 0.328 & 0.839 & 0.986 \\
\hline 45 & 0.406 & 0.852 & 0.981 \\
\hline 60 & 0.392 & 0.895 & 0.989 \\
\hline 90 & 0.428 & 0.968 & 0.995 \\
\hline 120 & 0.412 & 0.988 & 0.991 \\
\hline 150 & 0.419 & 0.993 & 1.000 \\
\hline 180 & 0.495 & 1.000 & 1.000 \\
\hline
\end{tabular}

Table C4.17: Drop size distribution at 5 minutes for $15 \mathrm{wt} \%$ PS solution dispersed phase at different stirring speed

\begin{tabular}{|c|c|c|c|c|c|c|}
\hline \multirow[b]{3}{*}{ Bin } & \multicolumn{6}{|c|}{ Frequency at different stirring speed } \\
\hline & \multicolumn{2}{|c|}{$350 \mathrm{rpm}$} & \multicolumn{2}{|l|}{$500 \mathrm{rpm}$} & \multicolumn{2}{|l|}{$650 \mathrm{rpm}$} \\
\hline & coloured & uncoloured & coloured & uncoloured & coloured & uncoloured \\
\hline 20 & 3 & 16 & 9 & 124 & 46 & 156 \\
\hline 40 & 33 & 21 & 24 & 117 & 106 & 130 \\
\hline 60 & 74 & 18 & 15 & 18 & 35 & 6 \\
\hline 80 & 87 & 13 & 7 & 0 & 0 & 0 \\
\hline 100 & 60 & 16 & 0 & 0 & 0 & 0 \\
\hline 120 & 26 & 7 & 0 & 0 & 0 & 0 \\
\hline 140 & 9 & 2 & 0 & 0 & 0 & 0 \\
\hline 160 & 3 & 7 & 0 & 0 & 0 & 0 \\
\hline 180 & 1 & 9 & 0 & $\overline{0}$ & 0 & 0 \\
\hline 200 & 1 & 3 & 0 & 0 & 0 & 0 \\
\hline 220 & 0 & 1 & 0 & 0 & 0 & 0 \\
\hline 240 & 0 & 2 & 0 & 0 & 0 & 0 \\
\hline More & 0 & 0 & 0 & 0 & 0 & 0 \\
\hline
\end{tabular}


Table C4.18: Drop size distribution at 180 minutes for $15 \mathrm{wt} \%$ PS solution dispersed phase at different stirring speed

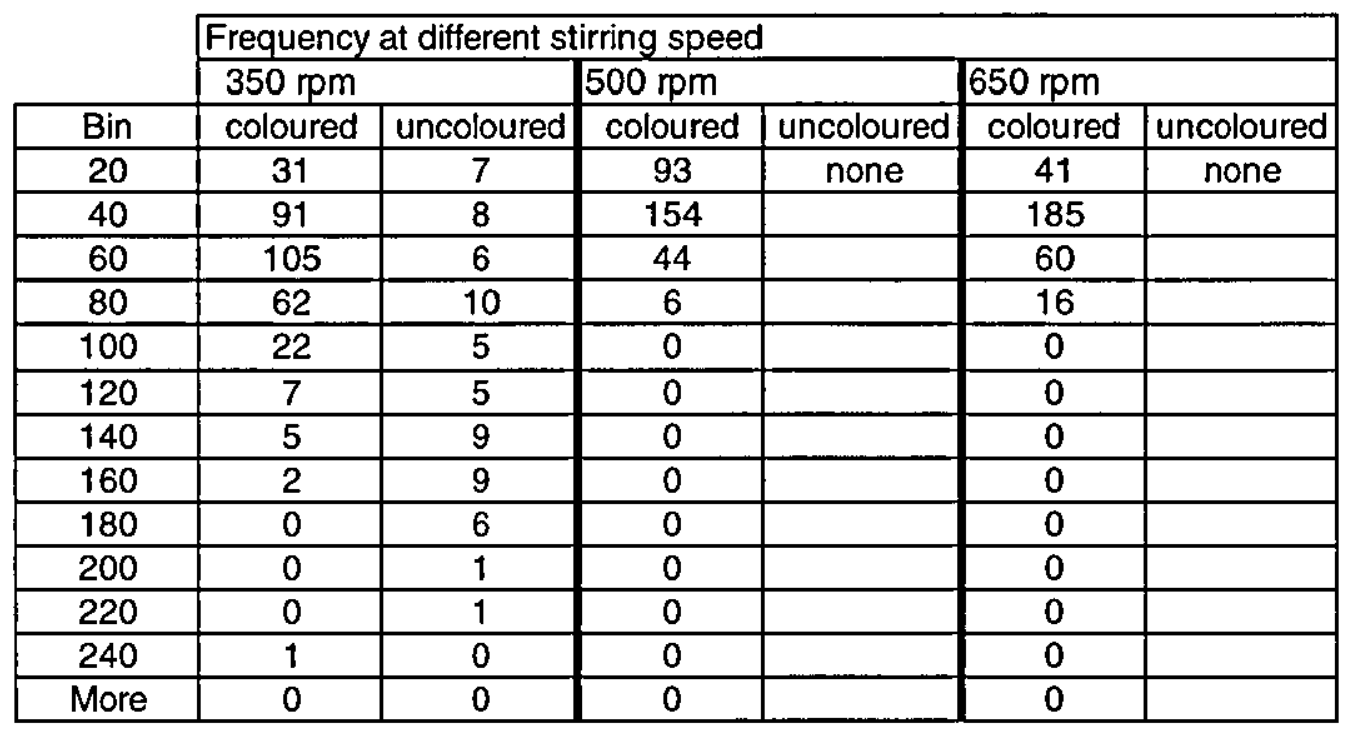

Table C4.19: Extent of mixing for batch mixing using 0.05 wt \% PVA at $500 \mathrm{rpm}$

\begin{tabular}{|c|c|c|}
\hline $\begin{array}{l}\text { Stirring time } \\
\text { (minutes) }\end{array}$ & \multicolumn{2}{|c|}{$\begin{array}{l}\text { Extent of mixing for } \\
\text { different concentration }\end{array}$} \\
\hline & $15 \%$ PS & $20 \%$ PS \\
\hline 0 & 0.200 & 0.200 \\
\hline 5 & 0.408 & 0.482 \\
\hline 15 & 0.488 & 0.681 \\
\hline 30 & 0.671 & 0.728 \\
\hline 45 & 0.729 & 0.788 \\
\hline 60 & 0.878 & 0.916 \\
\hline 90 & 0.904 & 0.973 \\
\hline 120 & 0.966 & 0.967 \\
\hline 150 & 0.970 & 0.995 \\
\hline 180 & 0.978 & 0.987 \\
\hline
\end{tabular}


Table C4.20: Extent of mixing for batch mixing using two different concentration of PVA at 15 wt \% PS

\begin{tabular}{|c|c|c|}
\hline $\begin{array}{l}\text { Stirring time } \\
\text { (minute) }\end{array}$ & \multicolumn{2}{|c|}{$\begin{array}{l}\text { Extent for mixing for different PVA } \\
\text { concentration }\end{array}$} \\
\hline & 0.02 wt\% PVA & 0.05 wt\% PVA \\
\hline 0 & 0.200 & 0.200 \\
\hline 5 & 0.514 & 0.408 \\
\hline 15 & 0.607 & 0.488 \\
\hline 30 & 0.839 & 0.671 \\
\hline 45 & 0.852 & 0.729 \\
\hline 60 & 0.895 & 0.878 \\
\hline 90 & 0.968 & 0.904 \\
\hline 120 & 0.988 & 0.966 \\
\hline 150 & 0.993 & 0.970 \\
\hline 180 & 1.000 & 0.978 \\
\hline
\end{tabular}

Table C4.21: Drop size distribution at 180 minutes of batch mixing for two different concentration of PVA

\begin{tabular}{|c|c|c|c|c|}
\cline { 2 - 5 } \multicolumn{1}{c|}{} & \multicolumn{4}{c|}{ Frequency at different concentration of PVA } \\
\cline { 2 - 5 } \multicolumn{1}{c|}{} & 0.02 wt \% PVA & \multicolumn{2}{c|}{0.05 wt \% PVA } \\
\hline Bin & coloured & uncoloured & coloured & uncoloured \\
\hline 10 & 35 & 16 & 14 & 30 \\
\hline 20 & 58 & 21 & 154 & 29 \\
\hline 30 & 78 & 18 & 91 & 6 \\
\hline 40 & 76 & 13 & 66 & 1 \\
\hline 50 & 26 & 16 & 26 & 0 \\
\hline 60 & 18 & 7 & 3 & 0 \\
\hline 70 & 4 & 2 & 4 & 0 \\
\hline 80 & 2 & 7 & 1 & 0 \\
\hline 90 & 0 & 9 & 0 & 0 \\
\hline 100 & 0 & 3 & 0 & 0 \\
\hline More & 0 & 1 & 0 & 0 \\
\hline
\end{tabular}


Table C4.22: Extent of mixing for batch mixing using $0.02 \mathrm{wt} \%$ Tween 20 for different viscosity of dispersed phase

\begin{tabular}{|c|c|c|c|}
\hline \multirow{2}{*}{$\begin{array}{l}\text { Time } \\
\text { (minutes) }\end{array}$} & \multicolumn{4}{|c|}{ Extent of mixing at different concentration PS } \\
\cline { 2 - 4 } & 5 wt\% PS & 10 wt\% PS & 15 wt\%PS \\
\hline 0 & 0.200 & 0.200 & 0.200 \\
\hline 5 & 0.250 & 0.254 & 0.245 \\
\hline 15 & 0.367 & 0.351 & 0.482 \\
\hline 30 & 0.465 & 0.519 & 0.564 \\
\hline 45 & 0.521 & 0.541 & 0.589 \\
\hline 60 & 0.512 & 0.563 & 0.660 \\
\hline 90 & 0.593 & 0.634 & 0.710 \\
\hline 120 & 0.630 & 0.646 & 0.816 \\
\hline 150 & 0.734 & 0.745 & 0.877 \\
\hline 180 & 0.763 & 0.800 & 0.890 \\
\hline
\end{tabular}

Table C4.23: Extent of mixing for batch mixing at $15 \mathrm{wt} \%$ PS dispersed phase using different type of surfactant

\begin{tabular}{|c|c|c|}
\hline \multirow{2}{*}{$\begin{array}{l}\text { Time } \\
\text { (minutes) }\end{array}$} & Extent of mixing for different surfactant \\
\cline { 2 - 3 } & 0.02 wt\% PVA & 0.02 wt\% Tween 20 \\
\hline 0 & 0.200 & 0.200 \\
\hline 5 & 0.514 & 0.245 \\
\hline 15 & 0.607 & 0.482 \\
\hline 30 & 0.839 & 0.564 \\
\hline 45 & 0.852 & 0.589 \\
\hline 60 & 0.895 & 0.660 \\
\hline 90 & 0.968 & 0.710 \\
\hline 120 & 0.988 & 0.816 \\
\hline 150 & 0.993 & 0.877 \\
\hline 180 & 1.000 & 0.890 \\
\hline
\end{tabular}


Table C4.24: Drop size distribution for batch mixing at 180 minutes using different surfactant

\begin{tabular}{|c|c|c|c|c|}
\cline { 2 - 5 } \multicolumn{1}{c|}{} & \multicolumn{4}{l|}{ Frequency at different surfactant } \\
\cline { 2 - 5 } \multicolumn{1}{c|}{} & 0.02 wt \% PVA & \multicolumn{2}{c|}{0.02 wt \% Tween 20 } \\
\hline Bin & coloured & uncoloured & coloured & uncoloured \\
\hline 20 & 93 & none & 15 & 16 \\
\hline 40 & 154 & & 97 & 14 \\
\hline 60 & 44 & & 93 & 11 \\
\hline 80 & 6 & & 34 & 8 \\
\hline 100 & 0 & & 44 & 11 \\
\hline 120 & 0 & & 61 & 5 \\
\hline 140 & 0 & & 19 & 3 \\
\hline 160 & 0 & & 11 & 2 \\
\hline 180 & 0 & & 5 & 0 \\
\hline 200 & 0 & & 1 & 0 \\
\hline More & 0 & & 0 & 0 \\
\hline
\end{tabular}


Table C4.25a: Drop size distribution for premixing after 120 minutes for $0 \mathrm{wt} \%$ polystyrene solution (styrene) dispersed phase

\begin{tabular}{|c|c|c|}
\cline { 2 - 3 } \multicolumn{1}{c|}{} & Frequency \\
\hline Bin & Coloured & Uncoloured \\
\hline 10 & 0 & 0 \\
\hline 20 & 3 & 16 \\
\hline 30 & 60 & 67 \\
\hline 40 & 121 & 97 \\
\hline 50 & 84 & 86 \\
\hline 60 & 84 & 94 \\
\hline 70 & 25 & 18 \\
\hline 80 & 9 & 6 \\
\hline 90 & 0 & 0 \\
\hline 100 & 0 & 0 \\
\hline 110 & 0 & 0 \\
\hline More & 0 & 0 \\
\hline
\end{tabular}

Table 4.25b: Drop size distribution for premixing after 120 minutes for $10 \mathrm{wt} \%$ polystyrene solution dispersed phase

\begin{tabular}{|c|c|c|}
\cline { 2 - 3 } \multicolumn{1}{c|}{} & \multicolumn{2}{c|}{ Frequency } \\
\hline Bin & Coloured & Uncoloured \\
\hline 20 & 29 & 36 \\
\hline 40 & 74 & 68 \\
\hline 60 & 62 & 61 \\
\hline 80 & 94 & 52 \\
\hline 100 & 64 & 77 \\
\hline 120 & 65 & 42 \\
\hline 140 & 28 & 12 \\
\hline 160 & 11 & 1 \\
\hline 180 & 0 & 0 \\
\hline 200 & 0 & 0 \\
\hline More & 0 & 0 \\
\hline
\end{tabular}


Table C4.26: Extent of mixing for mixing of two stabilised dispersions with different viscosities of dispersed phase

\begin{tabular}{|c|c|c|c|c|}
\hline \multirow{2}{*}{$\begin{array}{l}\text { Time } \\
\text { (minutes) }\end{array}$} & \multicolumn{4}{|c|}{ Extent of mixing for different concentration of dispersed phase } \\
\hline & $\begin{array}{l}0 w t \% \text { PS } \\
+0 w t \% \text { PS }\end{array}$ & $\begin{array}{l}5 w t \% \text { PS } \\
+5 w t \% \text { PS }\end{array}$ & $\begin{array}{l}10 w t \% \text { PS } \\
+10 w t \% \text { PS }\end{array}$ & $\begin{array}{l}15 w t \% \text { PS } \\
+15 w t \% \text { PS }\end{array}$ \\
\hline 0 & 0.500 & 0.500 & 0.500 & 0.500 \\
\hline 5 & 0.600 & 0.641 & 0.578 & 0.503 \\
\hline 15 & 0.636 & 0.661 & 0.624 & 0.513 \\
\hline 30 & 0.642 & 0.658 & 0.633 & 0.511 \\
\hline 45 & 0.683 & 0.718 & 0.632 & 0.509 \\
\hline 60 & 0.676 & 0.748 & 0.644 & 0.533 \\
\hline 90 & 0.727 & 0.755 & 0.689 & 0.545 \\
\hline 120 & 0.713 & 0.745 & 0.690 & 0.574 \\
\hline 150 & 0.722 & 0.762 & 0.676 & 0.576 \\
\hline 180 & 0.799 & 0.811 & 0.718 & 0.581 \\
\hline
\end{tabular}

Table C4.27: Drop size distribution at 5 minutes at different viscosity of dispersed phase for mixing of two dispersions

\begin{tabular}{|c|c|c|c|c|}
\hline & \multicolumn{4}{|c|}{ Frequency at different concentration of PS } \\
\hline & \multicolumn{2}{|c|}{$0 w t \% P S+0 w t \% P S$} & \multicolumn{2}{|c|}{$5 w t \% P S+5 w t \%$ PS } \\
\hline Bin & coloured & uncoloured & coloured & uncoloured \\
\hline 20 & 13 & 15 & 2 & 2 \\
\hline 40 & 135 & 90 & 43 & 43 \\
\hline 60 & 120 & 87 & 74 & 70 \\
\hline 80 & 41 & 25 & 75 & 61 \\
\hline 100 & 2 & 1 & 29 & 5 \\
\hline 120 & 0 & 0 & 3 & 0 \\
\hline 140 & 0 & 0 & 0 & 0 \\
\hline 160 & 0 & 0 & 0 & 0 \\
\hline 180 & 0 & 0 & 0 & 0 \\
\hline 200 & 0 & 0 & 0 & 0 \\
\hline \multirow[t]{3}{*}{ More } & 0 & 0 & 0 & 0 \\
\hline & \multicolumn{4}{|c|}{ Frequency at different concentration of PS } \\
\hline & \multicolumn{2}{|c|}{$10 w t \% P S+10 w t \%$ PS } & \multicolumn{2}{|c|}{15 wt $\%$ PS + $15 w t \%$ PS } \\
\hline Bin & coloured & uncoloured & coloured & uncoloured \\
\hline 20 & 21 & 27 & 6 & 1 \\
\hline 40 & 51 & 34 & 55 & 46 \\
\hline 60 & 43 & 20 & 47 & 23 \\
\hline 80 & 39 & 28 & 33 & 20 \\
\hline 100 & 54 & 42 & 34 & 18 \\
\hline 120 & 28 & 25 & 15 & 9 \\
\hline 140 & 10 & 7 & 9 & 20 \\
\hline 160 & 1 & 0 & $\overline{14}$ & 15 \\
\hline 180 & 0 & 0 & 18 & 16 \\
\hline 200 & 0 & 0 & 7 & 8 \\
\hline More & 0 & 0 & 0 & 0 \\
\hline
\end{tabular}


Table C4.28: Drop size distribution at 180 minutes at different viscosity of dispersed phase for mixing of two dispersion

\begin{tabular}{|c|c|c|c|c|}
\cline { 2 - 5 } \multicolumn{1}{c|}{} & \multicolumn{5}{c|}{ Frequency at different concentration of PS } \\
\hline & 0 wt \% PS +0 wt\% PS & 5 wt \% PS +5 wt\% PS \\
\hline Bin & coloured & uncoloured & coloured & uncoloured \\
\hline 20 & 5 & 1 & 3 & 27 \\
\hline 40 & 170 & 48 & 68 & 48 \\
\hline 60 & 195 & 60 & 102 & 45 \\
\hline 80 & 28 & 5 & 68 & 14 \\
\hline 100 & 2 & 0 & 14 & 1 \\
\hline 120 & 0 & 0 & 1 & 0 \\
\hline 140 & 0 & 0 & 0 & 0 \\
\hline 160 & 0 & 0 & 0 & 0 \\
\hline 180 & 0 & 0 & 0 & 0 \\
\hline 200 & 0 & 0 & 0 & 0 \\
\hline More & 0 & 0 & 0 & 0 \\
\hline & Frequency at different concentration of PS & \\
\hline & 10 wt \% PS +10 wt\% PS & 15 wt \% PS +15 wt\% PS \\
\hline Bin & coloured & uncoloured & coloured & uncoloured \\
\hline 20 & 20 & 31 & 0 & 1 \\
\hline 40 & 84 & 25 & 14 & 7 \\
\hline 60 & 73 & 45 & 39 & 11 \\
\hline 80 & 77 & 37 & 49 & 10 \\
\hline 100 & 46 & 18 & 43 & 13 \\
\hline 120 & 12 & 5 & 41 & 15 \\
\hline 140 & 4 & 0 & 33 & 11 \\
\hline 160 & 0 & 0 & 12 & 28 \\
\hline 180 & 0 & 0 & 3 & 9 \\
\hline 200 & 0 & 0 & 5 & 1 \\
\hline More & 0 & 0 & 0 & 0 \\
\hline
\end{tabular}


Table C4.29: Drop size distribution for mixing of two stabilised dispersion of styrene ( 0 wt \% PS) dispersed phase

\begin{tabular}{|c|c|c|c|c|c|c|}
\hline \multirow[b]{3}{*}{ Bin } & \multicolumn{6}{|c|}{ Frequency at different time } \\
\hline & \multicolumn{2}{|c|}{5 minutes } & \multicolumn{2}{|c|}{15 minutes } & \multicolumn{2}{|l|}{30 minutes } \\
\hline & coloured & uncoloured & coloured & uncoloured & coloured & uncoloured \\
\hline 10 & 0 & 0 & 0 & 0 & 0 & 0 \\
\hline 20 & 13 & 15 & 7 & 10 & 2 & 4 \\
\hline 30 & 52 & 39 & 44 & 34 & 32 & 29 \\
\hline 40 & 83 & 51 & 108 & 63 & 92 & 46 \\
\hline 50 & 63 & 39 & 93 & 70 & 89 & 59 \\
\hline 60 & 57 & 48 & 84 & 52 & 112 & 67 \\
\hline 70 & 32 & 21 & 18 & 8 & 19 & 25 \\
\hline 80 & 9 & 4 & 8 & 2 & 21 & 10 \\
\hline 90 & 2 & 1 & 2 & 0 & 2 & 0 \\
\hline 100 & 0 & 0 & 0 & 0 & 3 & 0 \\
\hline 110 & 0 & 0 & 0 & 0 & 0 & 0 \\
\hline \multirow[t]{3}{*}{ More } & 0 & 0 & 0 & 0 & 0 & 0 \\
\hline & \multicolumn{6}{|c|}{ Frequency at different time } \\
\hline & \multicolumn{2}{|c|}{45 minutes } & \multicolumn{2}{|l|}{60 minutes } & \multicolumn{2}{|l|}{90 minutes } \\
\hline Bin & coloured & uncoloured & coloured & uncoloured & coloured & uncoloured \\
\hline 10 & 0 & 1 & 0 & 0 & 0 & 1 \\
\hline 20 & 10 & 19 & 8 & 8 & 8 & 13 \\
\hline 30 & 44 & 23 & 65 & 35 & 44 & 30 \\
\hline 40 & 70 & 32 & 113 & 54 & 100 & 40 \\
\hline 50 & 81 & 62 & 88 & 36 & 120 & 55 \\
\hline 60 & 87 & 35 & 84 & 44 & 91 & 40 \\
\hline 70 & 39 & 21 & 16 & 4 & 31 & 8 \\
\hline 80 & 10 & 3 & 4 & 0 & 7 & 2 \\
\hline 90 & 5 & 0 & 0 & 2 & 2 & 0 \\
\hline 100 & 0 & 0 & 0 & 0 & 0 & 0 \\
\hline 110 & $\mathbf{0}$ & 0 & 0 & 0 & 1 & 0 \\
\hline \multirow[t]{3}{*}{ More } & 0 & 0 & 0 & 0 & 0 & 0 \\
\hline & \multicolumn{6}{|c|}{ Frequency at different time } \\
\hline & 120 minute & & 150 minutes & & 180 minutes & \\
\hline Bin & coloured & uncoloured & coloured & uncoloured & coloured & uncoloured \\
\hline 10 & 0 & 0 & 1 & 1 & 0 & 0 \\
\hline 20 & 19 & 26 & 16 & 16 & 5 & 1 \\
\hline 30 & 46 & 24 & 65 & 28 & 61 & 16 \\
\hline 40 & 66 & 30 & 90 & 42 & 109 & 32 \\
\hline 50 & 62 & 28 & 95 & 45 & 115 & 41 \\
\hline 60 & 38 & 14 & 48 & 21 & 80 & 19 \\
\hline 70 & 15 & 8 & 15 & 3 & 21 & 4 \\
\hline 80 & 4 & 0 & 4 & 0 & 7 & 1 \\
\hline 90 & 0 & 0 & 0 & 0 & 2 & 0 \\
\hline 100 & 0 & 0 & 0 & 0 & 0 & 0 \\
\hline 110 & 0 & 0 & 0 & 0 & 0 & 0 \\
\hline More & 0 & 0 & 0 & 0 & 0 & 0 \\
\hline
\end{tabular}


Table C4.30: Drop size distribution for mixing of two stabilised dispersion of $10 \mathrm{wt}$ $\%$ polystyrene solution dispersed phase

\begin{tabular}{|c|c|c|c|c|c|c|}
\hline \multirow[b]{3}{*}{ Bin } & \multicolumn{6}{|c|}{ Frequency at different time } \\
\hline & \multicolumn{2}{|c|}{5 minutes } & \multicolumn{2}{|c|}{15 minutes } & \multicolumn{2}{|l|}{30 minutes } \\
\hline & coloured & uncoloured & coloured & uncoloured & coloured & uncoloured \\
\hline 20 & 21 & 27 & 28 & 31 & 22 & 30 \\
\hline 40 & 51 & 34 & 62 & 39 & 68 & 27 \\
\hline 60 & 43 & 20 & 41 & 27 & 62 & 58 \\
\hline 80 & 39 & 28 & 37 & 24 & 23 & 28 \\
\hline 100 & 54 & 42 & 41 & 29 & 22 & 20 \\
\hline 120 & 28 & 25 & 23 & 18 & 21 & 7 \\
\hline 140 & 10 & 7 & 9 & 0 & 13 & 7 \\
\hline 160 & 1 & 0 & 0 & 0 & 0 & 0 \\
\hline 180 & 0 & 0 & 0 & 0 & 1 & 0 \\
\hline 200 & 0 & 0 & 0 & 0 & 0 & 0 \\
\hline \multirow[t]{3}{*}{ More } & 0 & 0 & 0 & 0 & 0 & 0 \\
\hline & \multicolumn{6}{|c|}{ Frequency at different time } \\
\hline & \multicolumn{2}{|c|}{45 minutes } & \multicolumn{2}{|c|}{60 minutes } & \multicolumn{2}{|c|}{90 minutes } \\
\hline Bin & coloured & uncoloured & coloured & uncoloured & coloured & uncoloured \\
\hline 20 & 33 & 42 & 11 & 7 & 10 & 12 \\
\hline 40 & 76 & 27 & 75 & 22 & 31 & 19 \\
\hline 60 & 40 & 19 & 68 & 19 & 44 & 18 \\
\hline 80 & 36 & 31 & 49 & 46 & 46 & 23 \\
\hline 100 & 45 & 46 & 51 & 36 & 34 & 26 \\
\hline 120 & 32 & 12 & 21 & 9 & 30 & 11 \\
\hline 140 & 7 & 3 & 5 & 1 & 2 & 0 \\
\hline 160 & 2 & 0 & 0 & 0 & 1 & 0 \\
\hline 180 & 0 & 0 & 0 & 0 & 1 & 0 \\
\hline 200 & 0 & 0 & 0 & 0 & 0 & 0 \\
\hline \multirow[t]{3}{*}{ More } & 0 & 0 & 0 & 0 & 0 & 0 \\
\hline & \multicolumn{6}{|c|}{ Frequency at different time } \\
\hline & 120 minute & & 150 minute & & 180 minute & \\
\hline Bin & coloured & uncoloured & coloured & |uncoloured & coloured & uncoloured \\
\hline 20 & 10 & 27 & 18 & 10 & 20 & 31 \\
\hline 40 & 77 & 21 & 92 & 50 & 84 & 25 \\
\hline 60 & 65 & 17 & 74 & 27 & 73 & 45 \\
\hline 80 & 53 & 29 & 53 & 47 & 77 & 37 \\
\hline 100 & 63 & 40 & 50 & 29 & 46 & 18 \\
\hline 120 & 31 & 12 & 19 & 2 & 12 & 5 \\
\hline 140 & 6 & 0 & 1 & 0 & 4 & 0 \\
\hline 160 & 0 & 0 & 0 & 0 & 0 & 0 \\
\hline 180 & 0 & 0 & 0 & 0 & 0 & 0 \\
\hline 200 & 0 & 0 & 0 & 0 & 0 & 0 \\
\hline More & 0 & 0 & 0 & 0 & 0 & 0 \\
\hline
\end{tabular}


Table C4.31: Extent of mixing for dispersed phase and batch mixing ( 0 wt $\%$ PS + $0 \mathrm{wt} \% \mathrm{PS}$ )

\begin{tabular}{|c|c|c|}
\hline \multirow{2}{*}{$\begin{array}{l}\text { Stirring } \\
\text { Time } \\
\text { (minutes) }\end{array}$} & \multicolumn{2}{|l|}{ Extent of mixing } \\
\hline & $\begin{array}{l}\text { Mixing of two stabilised } \\
\text { dispersion }\end{array}$ & $\begin{array}{l}\text { Mixing of unstabilised } \\
\text { to stabilised dispersion }\end{array}$ \\
\hline 0 & 0.500 & 0.500 \\
\hline 5 & 0.600 & 0.698 \\
\hline 15 & 0.636 & 0.706 \\
\hline 30 & 0.642 & 0.704 \\
\hline 45 & 0.683 & 0.702 \\
\hline 60 & 0.676 & 0.698 \\
\hline 90 & 0.727 & 0.739 \\
\hline 120 & 0.713 & 0.788 \\
\hline 150 & 0.722 & 0.794 \\
\hline 180 & 0.799 & 0.836 \\
\hline
\end{tabular}

Table C4.32: Drop size distribution for batch mixing of $0 \mathrm{wt} \%+0 \mathrm{wt} \%$

\begin{tabular}{|c|c|c|c|c|c|c|}
\cline { 2 - 7 } \multicolumn{1}{c|}{} & Frequency at different time & \multicolumn{3}{l|}{} \\
\cline { 2 - 7 } \multicolumn{1}{c|}{} & 5 minutes & \multicolumn{15}{l|}{ minutes } & 30 minutes \\
\hline Bin & coloured & uncoloured & coloured & uncoloured & coloured & uncoloured \\
\hline 10 & 0 & 1 & 0 & 0 & 0 & 0 \\
\hline 20 & 3 & 8 & 8 & 26 & 1 & 2 \\
\hline 30 & 16 & 26 & 11 & 20 & 20 & 12 \\
\hline 40 & 32 & 40 & 34 & 42 & 54 & 53 \\
\hline 50 & 22 & 46 & 18 & 24 & 42 & 31 \\
\hline 60 & 36 & 59 & 30 & 39 & 32 & 56 \\
\hline 70 & 31 & 31 & 19 & 32 & 33 & 19 \\
\hline 80 & 23 & 21 & 44 & 19 & 49 & 12 \\
\hline 90 & 22 & 1 & 22 & 4 & 18 & 1 \\
\hline 100 & 22 & 1 & 25 & 1 & 7 & 0 \\
\hline 110 & 3 & 1 & 3 & 0 & 0 & 0 \\
\hline 120 & 6 & 1 & 0 & 0 & 0 & 0 \\
\hline 130 & 4 & 0 & 0 & 0 & 0 & 0 \\
\hline 140 & 2 & 0 & 1 & 0 & 0 & 0 \\
\hline More & 0 & 0 & 0 & 0 & 0 & 0 \\
\hline
\end{tabular}

(continue next page) 
Continued from previous page

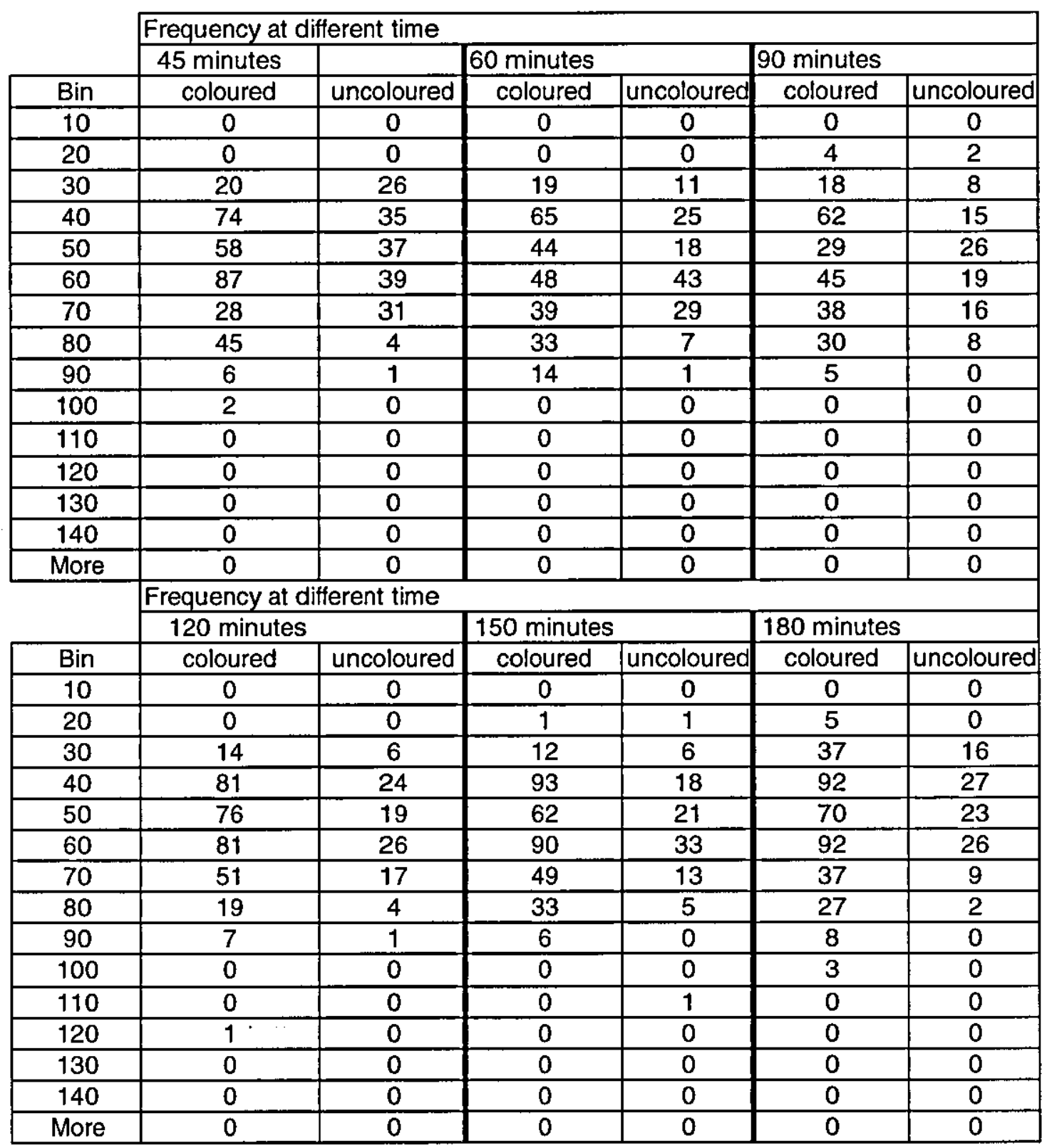


Table C4.33: Extent of mixing for dispersed phase and batch mixing ( $10 \mathrm{wt} \% \mathrm{PS}+$ $10 \mathrm{wt} \% \mathrm{PS}$ )

\begin{tabular}{|c|c|c|}
\hline \multirow{2}{*}{$\begin{array}{l}\text { Time } \\
\text { (time) }\end{array}$} & \multicolumn{2}{|l|}{ Extent of mixing } \\
\hline & $\begin{array}{c}\text { Mixing of two } \\
\text { stabilised dispersions }\end{array}$ & $\begin{array}{l}\text { Mixing of unstabilised } \\
\text { to stabilised dispersion }\end{array}$ \\
\hline 0 & 0.500 & 0.500 \\
\hline 5 & 0.600 & 0.698 \\
\hline 15 & 0.636 & 0.706 \\
\hline 30 & 0.642 & 0.704 \\
\hline 45 & 0.683 & 0.702 \\
\hline 60 & 0.676 & 0.698 \\
\hline 90 & 0.727 & 0.739 \\
\hline 120 & 0.713 & 0.788 \\
\hline 150 & 0.722 & 0.794 \\
\hline 180 & 0.799 & 0.836 \\
\hline
\end{tabular}


Table C4.34: Drop size distribution for batch mixing of $10 \mathrm{wt} \%+10 \mathrm{wt} \%$

\begin{tabular}{|c|c|c|c|c|c|c|}
\hline \multirow[b]{3}{*}{ Bin } & \multicolumn{6}{|c|}{ Frequency at different time } \\
\hline & \multicolumn{2}{|c|}{5 minutes } & \multicolumn{2}{|l|}{15 minutes } & \multicolumn{2}{|l|}{30 minutes } \\
\hline & coloured & uncoloured & coloured & uncoloured & coloured & uncoloured \\
\hline 20 & 6 & 8 & 8 & 9 & 5 & 0 \\
\hline 40 & 59 & 23 & 110 & 31 & 75 & 14 \\
\hline 60 & 70 & 27 & 114 & 28 & 112 & 32 \\
\hline 80 & 97 & 25 & 106 & 28 & 84 & 30 \\
\hline 100 & 48 & 33 & 38 & 24 & 33 & 19 \\
\hline 120 & 16 & 16 & 0 & 7 & 0 & 3 \\
\hline 140 & 0 & 4 & 0 & 0 & 0 & 3 \\
\hline 160 & 0 & 1 & 0 & 1 & 0 & 0 \\
\hline 180 & 0 & 0 & 0 & 0 & 0 & 0 \\
\hline 200 & 0 & 0 & 0 & 0 & 0 & 0 \\
\hline \multirow[t]{3}{*}{ More } & 0 & 0 & 0 & 0 & 0 & 0 \\
\hline & \multicolumn{6}{|c|}{ Frequency at different time } \\
\hline & 45 minutes & & \multicolumn{2}{|l|}{60 minutes } & \multicolumn{2}{|l|}{90 minutes } \\
\hline Bin & coloured & uncoloured & coloured & uncoloured & coloured & uncoloured \\
\hline 20 & 4 & 8 & 6 & 4 & 13 & 33 \\
\hline 40 & 95 & 23 & 101 & 40 & 112 & 32 \\
\hline 60 & 132 & 18 & 163 & 25 & 128 & 16 \\
\hline 80 & 124 & 11 & 87 & 29 & 78 & 14 \\
\hline 100 & 18 & 32 & 13 & 12 & 7 & 16 \\
\hline 120 & 2 & 9 & 3 & 5 & 1 & 2 \\
\hline 140 & 0 & 1 & 0 & 3 & 0 & 1 \\
\hline 160 & 0 & 0 & 0 & 0 & 0 & 0 \\
\hline 180 & 0 & 0 & 0 & 0 & 0 & 0 \\
\hline 200 & 0 & 0 & 0 & 0 & 0 & 0 \\
\hline \multirow[t]{3}{*}{ More } & $\begin{array}{l}0 \\
\end{array}$ & 0 & 0 & 0 & 0 & 0 \\
\hline & \multicolumn{6}{|c|}{ Frequency at different time } \\
\hline & \multicolumn{2}{|c|}{120 minutes } & \multicolumn{2}{|l|}{150 minutes } & \multicolumn{2}{|l|}{180 minutes } \\
\hline Bin & coloured & uncoloured & coloured & uncoloured & coloured & uncoloured \\
\hline 20 & 13 & 33 & 29 & 6 & 23 & 35 \\
\hline 40 & 112 & 32 & 112 & 42 & 135 & 33 \\
\hline 60 & 128 & 16 & 97 & 24 & 154 & 18 \\
\hline 80 & 78 & 14 & 38 & 16 & 52 & 10 \\
\hline 100 & 7 & 16 & 2 & 5 & 9 & 18 \\
\hline 120 & 1 & 2 & 0 & 0 & 1 & 3 \\
\hline 140 & 0 & 1 & 0 & 0 & 0 & 0 \\
\hline 160 & 0 & 0 & 0 & 0 & 0 & 0 \\
\hline 180 & 0 & 0 & 0 & 0 & 0 & 0 \\
\hline 200 & 0 & 0 & 0 & 0 & 0 & 0 \\
\hline More & 0 & 0 & 0 & 0 & 0 & 0 \\
\hline
\end{tabular}


Table C4.35: Extent of mixing for two dispersions of different viscosity of dispersed phase (a)

\begin{tabular}{|c|c|c|c|}
\hline \multirow{2}{*}{$\begin{array}{c}\text { Stirring } \\
\text { time } \\
\text { (minutes) }\end{array}$} & \begin{tabular}{c} 
Extent of mixing \\
\cline { 2 - 4 }+5 wt $\%$ PS
\end{tabular} & $\begin{array}{c}0 \text { wt } \% \text { PS } \\
+10 \text { wt \% PS }\end{array}$ & $\begin{array}{c}0 \text { wt\% PS + } \\
\text { 0 wt \% PS }\end{array}$ \\
\hline 0 & 0.500 & 0.500 & 0.500 \\
\hline 5 & 0.617 & 0.588 & 0.600 \\
\hline 15 & 0.644 & 0.623 & 0.636 \\
\hline 30 & 0.660 & 0.638 & 0.642 \\
\hline 45 & 0.694 & 0.650 & 0.683 \\
\hline 60 & 0.722 & 0.656 & 0.676 \\
\hline 90 & 0.730 & 0.695 & 0.727 \\
\hline 120 & 0.759 & 0.705 & 0.713 \\
\hline 150 & 0.754 & 0.705 & 0.722 \\
\hline 180 & 0.809 & 0.719 & 0.799 \\
\hline
\end{tabular}

Table C4.36: Extent of mixing for two dispersions of different viscosity of dispersed phase (b)

\begin{tabular}{|c|c|c|c|}
\hline \multirow{2}{*}{$\begin{array}{l}\text { Stirring } \\
\text { time } \\
\text { (minutes }\end{array}$} & \multicolumn{3}{|c|}{ Extent of mixing } \\
\hline & $\begin{array}{c}0 w \% \text { PS } \\
+5 w t \% \text { PS }\end{array}$ & $\begin{array}{r}5 \text { wt } \% \text { PS + } \\
5 \text { wt \% PS }\end{array}$ & $\begin{array}{c}0 w t \% \text { PS } \\
+0 w t \% \text { PS } \\
\end{array}$ \\
\hline 0 & 0.500 & 0.500 & 0.500 \\
\hline 5 & 0.617 & 0.641 & 0.600 \\
\hline 15 & 0.644 & 0.661 & 0.636 \\
\hline 30 & 0.660 & 0.658 & 0.642 \\
\hline 45 & 0.694 & 0.718 & 0.683 \\
\hline 60 & 0.722 & 0.748 & 0.676 \\
\hline 90 & 0.730 & 0.755 & 0.727 \\
\hline 120 & 0.759 & 0.745 & 0.713 \\
\hline 150 & 0.754 & 0.762 & 0.722 \\
\hline 180 & 0.809 & 0.811 & 0.799 \\
\hline
\end{tabular}

Table C4.37: Extent of mixing for two dispersions of different viscosity of dispersed phase (c)

\begin{tabular}{|c|c|c|c|}
\hline \multirow{2}{*}{$\begin{array}{l}\text { Stirring } \\
\text { time } \\
\text { (minutes }\end{array}$} & \multicolumn{3}{|c|}{ Extent of mixing } \\
\hline & $\begin{array}{c}0 w t \% \text { PS } \\
+10 w t \% \text { PS }\end{array}$ & $\begin{array}{c}10 w t \% \text { PS + } \\
10 \text { wt } \% \text { PS }\end{array}$ & $\begin{array}{c}0 w t \% \text { PS } \\
+10 w t \% \text { PS }\end{array}$ \\
\hline 0 & 0.500 & 0.500 & 0.500 \\
\hline 5 & 0.578 & 0.588 & 0.600 \\
\hline 15 & 0.624 & 0.623 & 0.636 \\
\hline 30 & 0.633 & 0.638 & 0.642 \\
\hline 45 & 0.632 & 0.650 & 0.683 \\
\hline 60 & 0.644 & 0.656 & 0.676 \\
\hline 90 & 0.689 & 0.695 & 0.727 \\
\hline 120 & 0.690 & 0.705 & 0.713 \\
\hline 150 & 0.676 & 0.705 & 0.722 \\
\hline 180 & 0.718 & 0.719 & 0.799 \\
\hline
\end{tabular}


Table C4.38: Drop size distribution for mixing of two stabilised dispersions ( 0 wt $\%$ PS + 5 wt $\%$ PS)

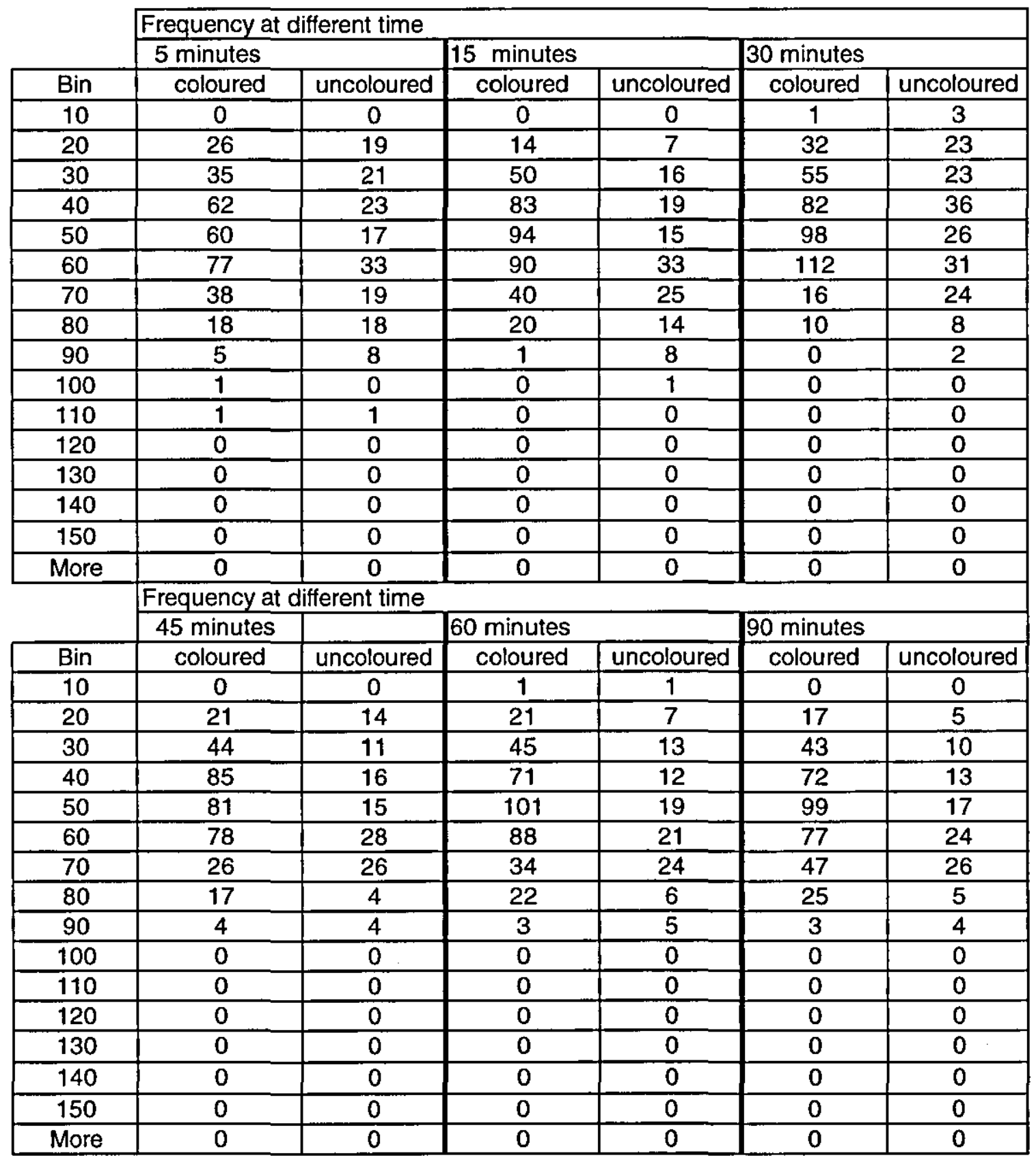

continue on next page 
Continued from previous page

\begin{tabular}{|c|c|c|c|c|c|c|}
\hline \multirow[b]{3}{*}{ Bin } & \multicolumn{6}{|c|}{ Frequency at different time } \\
\hline & \multicolumn{2}{|c|}{120 minutes } & \multicolumn{2}{|c|}{150 minutes } & \multicolumn{2}{|c|}{180 minutes } \\
\hline & coloured & uncoloured & coloured & uncoloured & coloured & uncoloured \\
\hline 10 & 0 & 0 & 0 & 0 & 1 & 0 \\
\hline 20 & 5 & 4 & 17 & 5 & 13 & 4 \\
\hline 30 & 33 & 8 & 42 & 10 & 55 & 17 \\
\hline 40 & 36 & 15 & 71 & 13 & 57 & 26 \\
\hline 50 & 113 & 17 & 104 & 17 & 122 & 15 \\
\hline 60 & 79 & 20 & 77 & 24 & 98 & 21 \\
\hline 70 & 29 & 22 & 48 & 26 & 53 & 19 \\
\hline 80 & 23 & 4 & 25 & 5 & 18 & 5 \\
\hline 90 & 3 & 1 & 2 & 2 & 5 & 0 \\
\hline 100 & $\mathbf{0}$ & 0 & 0 & 0 & 0 & 0 \\
\hline 110 & 0 & 0 & 0 & 0 & 0 & 0 \\
\hline 120 & 0 & 0 & 0 & 0 & 0 & 0 \\
\hline 130 & 0 & 0 & 0 & 0 & 0 & 0 \\
\hline 140 & 0 & 0 & 0 & 0 & 0 & 0 \\
\hline 150 & 0 & 0 & 0 & 0 & 0 & 0 \\
\hline More & 0 & 0 & 0 & 0 & 0 & 0 \\
\hline
\end{tabular}

Table C4.39: Drop size distribution for mixing of two stabilised dispersions ( $0 \mathrm{wt} \%$ PS + $10 \mathrm{wt} \%$ PS)

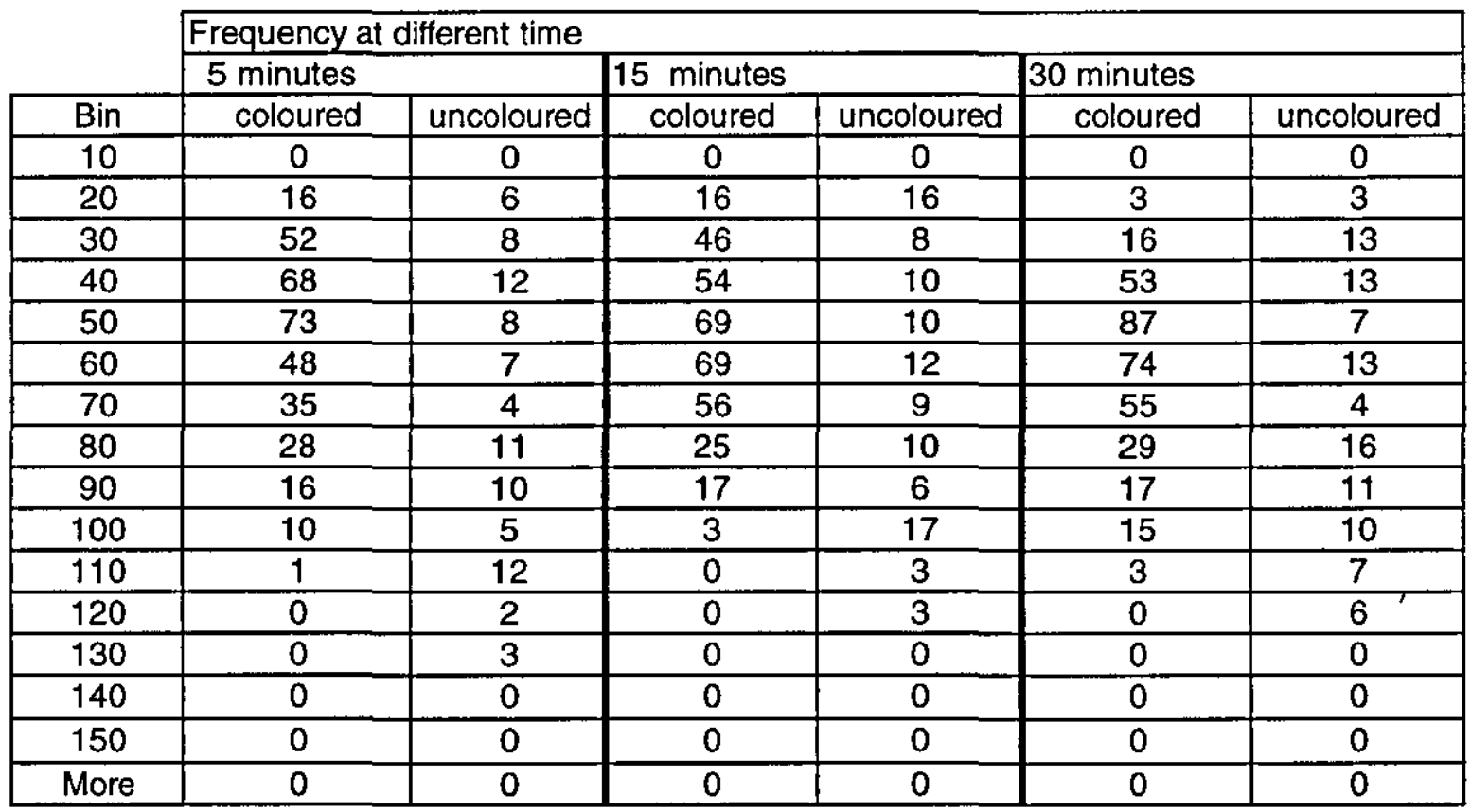

continue on next page 
continued from previous page

\begin{tabular}{|c|c|c|c|c|c|c|}
\hline & \multicolumn{6}{|c|}{ Frequency at different time } \\
\hline & 45 minutes & & \multicolumn{2}{|l|}{60 minutes } & \multicolumn{2}{|l|}{90 minutes } \\
\hline Bin & coloured & uncoloured & coloured & uncoloured & coloured & uncoloured \\
\hline 10 & 0 & 0 & 0 & 0 & 0 & 0 \\
\hline 20 & 3 & 3 & 5 & 3 & 10 & 8 \\
\hline 30 & 43 & 5 & 53 & 3 & 37 & 7 \\
\hline 40 & 75 & 10 & 72 & 11 & 70 & 16 \\
\hline 50 & 87 & 7 & 69 & 4 & 71 & 7 \\
\hline 60 & 68 & 7 & 49 & 10 & 95 & 17 \\
\hline 70 & 47 & 11 & 36 & 6 & 50 & 8 \\
\hline 80 & 29 & 9 & 25 & 5 & 16 & 5 \\
\hline 90 & 21 & 7 & 11 & 4 & 18 & 8 \\
\hline 100 & 24 & 5 & 6 & 3 & 7 & 7 \\
\hline 110 & 3 & 12 & 0 & 3 & 5 & 5 \\
\hline 120 & 0 & 10 & 0 & 7 & 1 & 3 \\
\hline 130 & 0 & 0 & 0 & 1 & 0 & 1 \\
\hline 140 & 0 & 0 & 0 & 0 & 0 & 0 \\
\hline 150 & 0 & 0 & 0 & 0 & 0 & 0 \\
\hline \multirow[t]{3}{*}{ More } & 0 & 0 & 0 & 0 & 0 & 0 \\
\hline & \multicolumn{6}{|c|}{ Frequency at different time } \\
\hline & \multicolumn{2}{|c|}{120 minutes } & \multicolumn{2}{|c|}{150 minutes } & \multicolumn{2}{|c|}{180 minutes } \\
\hline Bin & coloured & uncoloured & coloured & uncoloured & coloured & uncoloured \\
\hline 10 & 0 & 0 & 0 & 0 & 0 & 1 \\
\hline 20 & 10 & 7 & 26 & 15 & 24 & 9 \\
\hline 30 & 56 & 8 & 74 & 13 & 45 & 13 \\
\hline 40 & 106 & 10 & 152 & 26 & 113 & 7 \\
\hline 50 & 128 & 8 & 84 & 13 & 86 & 8 \\
\hline 60 & 79 & 13 & 59 & 12 & 59 & 11 \\
\hline 70 & 44 & 12 & 45 & 4 & 35 & 4 \\
\hline 80 & 31 & 16 & 18 & 7 & 19 & 13 \\
\hline 90 & 12 & 15 & 13 & 5 & 18 & 7 \\
\hline 100 & 3 & 5 & 2 & 5 & 16 & 10 \\
\hline 110 & 4 & 3 & 0 & 2 & 10 & 5 \\
\hline 120 & 2 & 0 & 0 & 3 & 4 & 3 \\
\hline 130 & 0 & 0 & 0 & 0 & 1 & 0 \\
\hline 140 & 0 & 0 & 0 & 0 & 0 & 0 \\
\hline 150 & 0 & 0 & 0 & 0 & 0 & 0 \\
\hline More & 0 & 0 & 0 & 0 & 0 & 0 \\
\hline
\end{tabular}




\section{APPENDIX D}

\section{Statistical analysis on the value of the extent of mixing}

In this section, statistical approach was used to determine the confidence interval of the value of the extent of mixing produced from the experiments. A confidence interval is a range of data values $(\mathrm{X})$ that encompasses some percentage of the total population. For normal distribution, when population mean and standard deviation are known, the confidence interval is expressed as:

$$
\mathrm{m}-\mathrm{Z} \alpha<\mathrm{X}<\mathrm{m}+\mathrm{Z} \alpha
$$

where: $\mathrm{m}=$ mean value

$\alpha=$ standard deviation

$\mathrm{Z}=$ number of standard deviation require to encompass some given probability

For a $95 \%$ confidence interval, $\mathrm{Z}$ is 1.96 which means that $95 \%$ of observed $\mathrm{X}$ values will fall within 1.96 standard deviations of the mean.

For smaller samples, it is better to use $t$ distribution, its parameter included the number of degree of freedom (sample size-1) as well as mean value and standard deviation. The number of standard deviations that encompass some percentage of the population depends on the degrees of freedom. This number, $t$, is analogous to $\mathrm{Z}$. The confidence interval is expressed as:

$$
\bar{X}-\text { ts }<X<\bar{X}+\text { ts }
$$

For $95 \%$ confidence interval with 5 samples ( 4 degree of freedom), $t$ is equal to 2.776 .

One set of experiment at certain condition has been chosen to determine the confidence interval. Five values of the extent of mixing were determined. To 
determine a value of extent of mixing, at least 300 drops were analysed. Therefore for 5 values of extent of mixing, at least 1500 drops were analysed. This amount is considered sufficient to represent the population mean and standard deviation. The experiments of batch mixing of $15 \mathrm{wt} \%$ polystyrene solution was chosen for this purposes. The analysis was done at two different times, 5 minutes and 60 minutes. Table D.1 shows the results of the analysis at 5 minutes and 60 minutes.

Table D.1: Statistical data for extent of mixing

\begin{tabular}{|l|c|c|c|c|c|l|l|}
\hline \multirow{2}{*}{ Time } & \multicolumn{4}{|c|}{ Extent of mixing } & Overall & Overall Standard \\
& Data 1 & Data 2 & Data 3 & Data 4 & Data 5 & average & Deviation \\
\hline $5 \mathrm{~min}$ & 0.513 & 0.505 & 0.500 & 0.513 & 0.492 & 0.505 & 0.008961 \\
\hline $60 \mathrm{~min}$ & 0.894 & 0.901 & 0.897 & 0.882 & 0.881 & 0.891 & 0.009028 \\
\hline
\end{tabular}

From the results in table D.1, confidence interval can be determined for $95 \%$.

For data at 5 minutes, confidence interval for extent of mixing is

$$
0.505 \pm 0.025
$$

And for data 60 minutes, confidence interval for extent of mixing is

$$
0.891 \pm 0.025
$$


